Felipe Iwao Takiyama

Algoritmos de Inferência Exata para Modelos de Primeira Ordem 
Felipe Iwao Takiyama

\section{Algoritmos de Inferência Exata para Modelos de Primeira Ordem}

Dissertação apresentada à Escola Politécnica da Universidade de São Paulo para obtenção do Título de Mestre em Engenharia Mecânica. 
Felipe Iwao Takiyama

\section{Algoritmos de Inferência Exata para Modelos de Primeira Ordem}

Dissertação apresentada à Escola Politécnica da Universidade de São Paulo para obtenção do Título de Mestre em Engenharia Mecânica.

Área de concentração: Engenharia de Controle e Automação Mecânica

Orientador:

Prof. Dr. Fabio Gagliardi Cozman 
FICHA CATALOGRÁFICA

Takiyama, Felipe Iwao

Algoritmos de inferência exata para modelos de primeira ordem / F.I. Takiyama. -- São Paulo, 2014. $131 \mathrm{p}$.

Dissertação (Mestrado) - Escola Politécnica da Universidade de São Paulo. Departamento de Engenharia Mecatrônica e de Sistemas Mecânicos.

1.Algoritmo de inferência exata 2.Algoritmo de eliminação de Variáveis em primeira ordem AC-FOVE I.Universidade de São Paulo. Escola Politécnica. Departamento de Engenharia Mecatrônica e de Sistemas Mecânicos II.t. 


\section{Resumo}

Este trabalho descreve a implementação de algoritmos de inferência para modelos de primeira ordem. Três algoritmos foram implementados: VE, C-F OVE e AC-F OVE. Este último é o estado da arte no cálculo de probabilidades em Redes Bayesianas Relacionais e não possuía nenhuma implementação disponível. O desenvolvimento foi feito segundo uma metodologia ágil que resultou em um pacote de software que pode ser utilizado em outras implementações. Mostra-se que o software criado possui o desempenho esperado em teoria, embora apresente algumas limitações. Esta dissertação contribui também com novos tópicos teóricos que complementam o algoritmo.

Palavras-chave: Algoritmo de inferência exata. Algoritmo de eliminação de variáveis em primeira ordem. AC-FOVE. 


\section{Abstract}

In this work, we describe the implementation of inference algorithms for first order models. Three algorithms were implemented: VE, C-FOVE and AC-FOVE. The latter is the state of the art in probability calculations for Relational Bayesian Networks and had no implementation available. The development was done according to an agile methodology, which resulted in a software that can be used in other packages. We show that the resulting software has the expected performance from the theory, although with some limitations. This work also contributes with new theoretical topics that complement the algorithm.

Keywords: Exact inference algorithm. First-order variable elimination algorithm. AC-FOVE. 


\section{Lista de Figuras}

1 Rede Bayesiana com 5 variáveis aleatórias. . . . . . . . . . . . . . . . . p. 19

2 Versão de primeira ordem da rede Sprinkler (MURPHY, 1998) . . . . . . . p. 30

3 Versão proposicionalizada da rede Sprinkler . . . . . . . . . . . . . . p. 30

4 Relação entre os conjuntos de fatores dos parfactors do Exemplo 13 . . . p. 33

5 Rede Bayesiana que representa uma relação entre apostadores e ganhadores p. 34

6 Modelo de primeira ordem equivalente à rede da Figura 5 . . . . . . . . p. 35

$7 \quad$ Rede Loteria proposta por Kisynski (2010) . . . . . . . . . . . . . . . . . p. 85

8 Metodologia XP (adaptado de (DZHUROV; KRASTEVA; ILIEVA, 2009)) . . p. 95

9 Código para definição de constante . . . . . . . . . . . . p. 96

10 Código para definição de variável lógica . . . . . . . . . . . . . p. 97

11 Código para definição de restrições . . . . . . . . . . . . . . . p. 97

12 Código para definição de uma variável aleatória parametrizada na forma

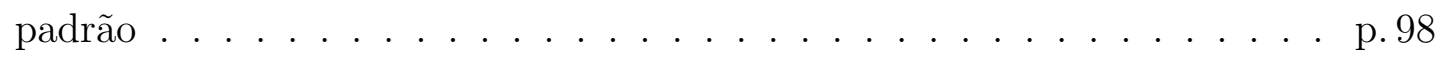

13 Código para definição de uma variável aleatória parametrizada booleana p. 98

14 Código para definição de uma fórmula de contagem . . . . . . . . . . . p. 98

15 Código para definição de um parfactor . . . . . . . . . . . . . p. 99

16 Código para definição de um parfactor de agregação simples . . . . . . . p. 101

17 Código para definição de parfactors de agregação mais complexos . . . . p. 101

18 Chamada de método inválido para parfactor de agregação . . . . . . . . p. 102

19 Código para converter um Parfactor em AggregationParfactor . . . . . . p. 102

20 Código para definição de uma consulta para o A C-FOVE . . . . . . . . . p. 103

21 Código para definição de um margina . . . . . . . . . . . . . . . . p. 103 
22 Código para definição de uma evidência . . . . . . . . . . . . . . . . p. 104

23 Código para executar o algoritmo AC-FOVE . . . . . . . . . . . . p. 104

24 Código para especificação de uma rede de primeira ordem e execução do algoritmo AC-FOVE . . . . . . . . . . . . . . . . p. 106

25 Representação em primeira ordem e proposicionalizada da rede do Exem-

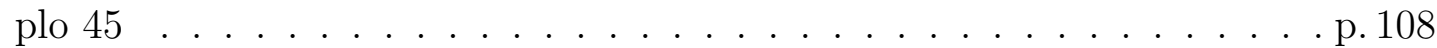

26 Representação proposicionalizada da rede do Exemplo 45, simplificada para o cálculo de $\mathcal{F}\left(a\left(x_{1}\right)\right) \ldots \ldots$. . . . . . . . . . . . . . 109

27 Representação em primeira ordem da rede da Figura 26 . . . . . . . . . . p. 109

28 Rede Sprinkler utilizada no experimento . . . . . . . . . . . . . . . . p. 112

29 Média do tempo de execução dos algoritmos de inferência para a rede Sprinkler (MURPHY, 1998) . . . . . . . . . . . . . . . . . p. 114

30 Rede Epidemia utilizada no experimento . . . . . . . . . . . . . p. 115

31 Média do tempo de execução dos algoritmos de inferência para a rede Epidemia (BRAZ; AMIR; ROTH, 2005) ․ . . . . . . . . . . . . . p. 116

32 Rede Workshops Concorrentes utilizada no experimento . . . . . . . . . p. 117

33 Média do tempo de execução dos algoritmos de inferência para a rede Workshops Concorrentes (MILCH et al., 2008) com 3 workshops . . . . . . p. 118

34 Média do tempo de execução dos algoritmos de inferência para a rede Workshops Concorrentes (MILCH et al., 2008) com 5 pessoas . . . . . . . . p. 119

35 Rede Loteria utilizada no experimento . . . . . . . . . . . . . . . p. 120

36 Média do tempo de execução dos algoritmos de inferência para a rede Loteria (KISYNSKI, 2010) . . . . . . . . . . . . . . . . p. 121

37 Representação das atribuições $P(A)=\alpha_{1}, P(B \mid A)=\alpha_{2}$ e $P(r)=\alpha_{3}$. . . p. 129

38 Representação da definição $A \equiv B$. . . . . . . . . . . . . . . . . p. 130

39 Representação da inclusão $A \sqsubseteq B \ldots$. . . . . . . . . . . . . . . . . p. 130

40 Representação da disjunção $C \equiv A \sqcup B$. . . . . . . . . . . . . . . . p. 131

41 Representação da conjunção $C \equiv A \sqcap B . \ldots$. . . . . . . . . . . . . p. 131 
42 Representação da negação $C \equiv \neg A$. . . . . . . . . . . . . . . . . p. 132

43 Representação da restrição universal $\forall r(x, y) . C(y)$. . . . . . . . . . . p. 133

44 Representação da restrição existencial $\exists r(x, y) . C(y) \ldots$. . . . . . . . . . . p. 134 


\section{Lista de Siglas}

- AC-F Ove: Algoritmo de Eliminação de Variáveis em Primeira Ordem com uso de Agregação e Contagem (KISYNSKI; POOLE, 2009).

- $\mathcal{A L C}$ : Attributive Concept Language with Complements, uma lógica de descrição básica (SCHMIDT-SCHAUSS; SMOLKA, 1991).

- API: Interface de Programação de Aplicativos, descreve a interface de uma biblioteca de componentes para que possam ser utilizados em outros softwares.

- BN: Rede Bayesiana.

- C-F Ove: Algoritmo de Eliminação de Variáveis em Primeira Ordem com Contagem (MILCH et al., 2008).

- cr $\mathcal{A L C}$ : Credal $\mathcal{A L C}$ (POLASTRO; COZMAn, 2008).

- FIFO: Esquema/política de filas (First In, First Out).

- GLBP: Algoritmo Generalizado de Propagação de Crença em Laço (Generalized Loopy Belief Propagation) (YEDIDIA; FREEMAN; WEISS, 2005).

- RBN: Rede Bayesiana Proposicional.

- TDD: Desenvolvimento Orientado a Testes (JANZEN; SAIEDIAN, 2005).

- VE: Algoritmo de Eliminação de Variáveis (ZHANG; POOLE, 1994). 


\section{Sumário}

1 Introdução $\quad$ p. 14

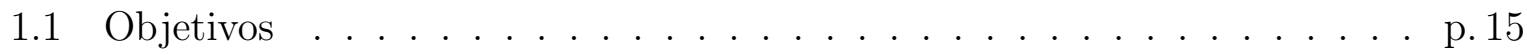

1.2 Organização do Texto . . . . . . . . . . . . . . . . p. 16

2 Redes Bayesianas p. 18

2.1 Redes Bayesianas . . . . . . . . . . . . . . . . . . p. 18

2.2 Inferência em Redes Bayesianas . . . . . . . . . . . . . . . . . p. 19

2.2 .1 Fatores . . . . . . . . . . . . . . . . p. 20

2.2.2 Algoritmo de Eliminação de Variáveis . . . . . . . . . . . . . . . p. 22

3 O Algoritmo AC-FOVE $\quad$ p. 24

3.1 Conceitos e definições . . . . . . . . . . . . . . . . . . . . . p. 24

3.1.1 Variáveis aleatórias parametrizadas . . . . . . . . . . p. 24

3.1 .2 Fórmulas de contagem . . . . . . . . . . . . p. 26

3.1 .3 Parfactors . . . . . . . . . . . . . . . . . p. 28

3.1.4 Atribuição de valores . . . . . . . . . . . . . . . . p. p. 33

3.1.5 Parfactors de agregação . . . . . . . . . . . . . . p. 34

3.1.6 Restrições na forma normal . . . . . . . . . . . . . . . . . . p. 38

3.2 Operações elementares . . . . . . . . . . . . . . . . . . . . . . . . p. 39

3.2 .1 Eliminação . . . . . . . . . . . . . . . . . . . . . p. . p 41

3.2 .2 Multiplicação . . . . . . . . . . . . . . . . . . . p. 49

3.2.3 Divisão e proposicionalização . . . . . . . . . . . . . p. 52 
3.2 .4 Expansão . . . . . . . . . . . . . . . . . . p. 57

3.2 .5 Conversão . . . . . . . . . . . . . . . . . . . p. 60

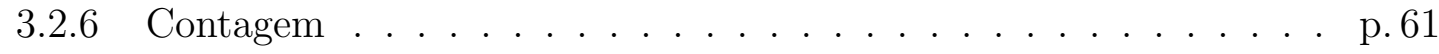

3.2 .7 Unificação . . . . . . . . . . . . . . . . . . p. 63

3.2.7.1 Pré-processamento . . . . . . . . . . . . p. 63

3.2.7.2 Unificador mais geral . . . . . . . . . . . . p. 65

3.2.7.3 Particionamento de conjuntos . . . . . . . . . . p. 68

3.2.7.4 Algoritmo de unificação completo . . . . . . . . . . . p. p. 70

3.3 Macro-operações . . . . . . . . . . . . . . . . . . p. 73

3.3.1 Particionamento. . . . . . . . . . . . . . p. 74

3.3.2 Eliminação global . . . . . . . . . . . . . . . . p. 75

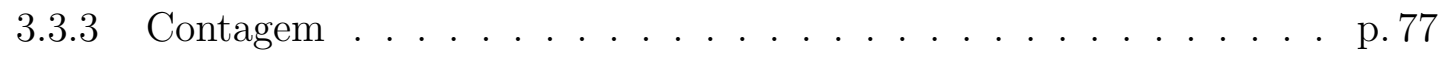

3.3.4 Proposicionalização . . . . . . . . . . . . . . . p. 78

3.3.5 Expansão total . . . . . . . . . . . . . . . . . . p. 80

3.3.6 Conversão . . . . . . . . . . . . . . . . . . . . . . p. 82

$3.4 \mathrm{O}$ algoritmo $\ldots \ldots \ldots \ldots \ldots \ldots \ldots \ldots \ldots \ldots$

4 Implementação $\quad$ p. 93

4.1 Metodologia . . . . . . . . . . . . . . . . p. 94

4.2 Fases do Projeto . . . . . . . . . . . . . . . . . . . p. 95

4.3 Utilização . . . . . . . . . . . . . . . . . . . . . . . . . . . p. 96

4.3.1 Definição de constantes . . . . . . . . . . . . . . . . . . p. 96

4.3.2 Definição de variáveis lógicas . . . . . . . . . . . . . . p. 96

4.3 .3 Definição de restrições . . . . . . . . . . . . . . . . p. 96

4.3.4 Definição de variáveis aleatórias parametrizadas . . . . . . . . . p.97

4.3.5 Definição de fórmulas de contagem . . . . . . . . . . . . p. 98

4.3.6 Definição de parfactors na forma padrão . . . . . . . . . . . . . . p. 99 
4.3.7 Definição de parfactors de agregação . . . . . . . . . . . . . . p. 100

4.3 .8 Definição de consulta . . . . . . . . . . . . . . . . . . p. 102

4.3 .9 Definição de marginais . . . . . . . . . . . . . . . . p. 103

4.3.10 Inclusão de evidências ～. . . . . . . . . . . . . . . . . . p. 103

4.3 .11 Execução do algoritmo . . . . . . . . . . . . . . . . . . . p. 104

4.3 .12 Extensão do algoritmo . . . . . . . . . . . . . . . . p. 104

5 Eliminação em parfactors de agregação $\quad$ p. 107

5.1 Novo método de eliminação . . . . . . . . . . . . . . . . . . p. 108

6 Experimentos $\quad$ p. 111

6.1 Rede Sprinkler . . . . . . . . . . . . . . . . . . . . . p. 112

6.2 Rede Epidemia . . . . . . . . . . . . . . . . . . . p. 114

6.3 Rede Workshops Concorrentes . . . . . . . . . . . . . . . p. 116

6.4 Rede Loteria . . . . . . . . . . . . . . . . . . . . . . . . . . . . . . p. 119

6.5 Análise dos Resultados . . . . . . . . . . . . . . . . p. 121

7 Considerações finais $\quad$ p. 123

$\begin{array}{ll}\text { Referências } & \text { p. } 125\end{array}$

Apêndice A - Credal ALC e Parfactors $\quad$ p. 128

A.1 Atribuições de probabilidade . . . . . . . . . . . . . . . . p. 129

A.2 Definição e inclusão de conceitos . . . . . . . . . . . . . . . . . p. 129

A.3 Construtores lógicos . . . . . . . . . . . . . . . . . p. 130

A.4 Restrições universais . . . . . . . . . . . . . . . . . . . p. 131

A.5 Restrições existenciais . . . . . . . . . . . . . . . . . . . . . p. 132 


\section{Introdução}

Uma das vertentes da Inteligência Artificial trata do projeto e da construção de agentes que agem de maneira inteligente e racional (RUSSELL; NORVIG, 1995). Tais agentes devem ser capazes de lidar com as incertezas geradas pelas restrições nas informações disponibilizadas, visto que em situações reais não é possível obter uma visão completa do mundo.

A teoria de probabilidades fornece os alicerces para a criação de modelos de representação e sistemas de raciocínio diante de incertezas. Dado um modelo da realidade, uma tarefa comum é calcular a probabilidade de uma variável aleatória assumir um determinado valor dado o valor de outras variáveis aleatórias. Ao realizar raciocínios no mundo real, um agente pode precisar lidar com domínios com um número muito grande de indivíduos. Embora o agente possa estar interessado em apenas alguns deles, é preciso considerar a influência exercida pelos outros nos cálculos.

Para ilustrar a influência do tamanho do domínio, considere que foi encontrado um veículo cujas características são consistentes com a descrição informada em uma ocorrência de roubo relatada no dia anterior. Qual a probabilidade do veículo ter sido roubado? Um dos fatores a se considerar é quão bem a descrição relatada se encaixa com a do veículo observado. Além disso, se a cidade onde ocorreu o roubo é pequena e isolada, é muito provável que o carro em questão tenha sido roubado. Entretanto, se a cidade for grande, a probabilidade do carro encontrado ter sido roubado diminui. Assim, muito embora não exista informação sobre todos os outros veículos da cidade, estes afetam o cálculo da probabilidade de interesse.

Modelos gráficos probabilísticos, tais como Redes Bayesianas (PEARL, 1988), são ferramentas populares para representação de relações entre variáveis aleatórias. Por serem representações de ordem zero (proposicionais), elas são inadequadas para descrever relações entre indivíduos ou quantificar sobre conjuntos, pois para representar cada propriedade de cada indivíduo é necessário criar uma variável aleatória. Por outro lado, lógicas de 
primeira ordem permitem representar relações e quantificar sobre variáveis lógicas, sendo no entanto incapazes de tratar incertezas.

Modelos gráficos que unem os conceitos de lógicas de primeira ordem e probabilidades já foram propostos há mais de vinte anos (BREESE, 1992) (HORSCH; POOLE, 1990) e outros têm surgido (RAEDT et al., 2008) (GETOOR; TASKAR, 2007). Neste modelos, as variáveis aleatórias são parametrizadas por variáveis lógicas atreladas a uma população, o que permite representar de maneira compacta a mesma informação para vários indivíduos e explorar a representação para realizar inferências mais eficientes.

Uma das técnicas mais simples para realizar inferências em modelos de primeira ordem consiste em proposicionalizar a parte relevante para a consulta e em seguida aplicar um algoritmo de inferência neste "pedaço". O problema desta abordagem é que ela se torna computacionalmente inviável mesmo para modelos mais simples. A ideia da inferência em primeira ordem é realizar os cálculos evitando ao máximo proposicionalizar o modelo.

Poole (2003) foi o primeiro a propor um algoritmo de inferência exata para modelos de primeira ordem. Braz, Amir e Roth (2005, 2006, 2007) melhoraram o algoritmo e estenderam o trabalho para modelos não direcionados. Em seguida, Milch et al. (2008) aumentaram a eficiência do algoritmo adicionando estruturas denominadas fórmulas de contagem. O algoritmo resultante foi denominado C-F OvE. Finalmente, Kisynski e Poole (2009) propuseram a inclusão de agregação e formas de tratá-la para aumentar o desempenho do algoritmo, resultando no algoritmo conhecido como AC-FOVE. Atualmente, o este algoritmo é estado da arte em inferência exata para modelos de primeira ordem. Embora a teoria do algoritmo tenha sido formalizada no doutorado de Kisynski (2010), não se conhecia nenhuma implementação publicamente disponível para o algoritmo.

\subsection{Objetivos}

O objetivo deste trabalho é implementar o algoritmo AC-F OVE (KISYNSKI; POOLE, 2009) de maneira que possa ser utilizado para realizar inferências em modelos de primeira ordem.

Ressalta-se que, embora os modelos de primeira ordem utilizados neste trabalho utilizem conceitos de lógica de primeira ordem, a realização de provas lógicas está fora do escopo deste trabalho. O termo "inferência" é utilizado para denotar o cálculo de probabilidades em redes Bayesianas relacionais. 
Inicialmente, planejou-se aplicar este algoritmo à lógica de descrição probabilística Credal $\mathcal{A L C}$, proposta por Polastro e Cozman (2008). Entretanto, devido a algumas limitações encontradas no algoritmo (vide Capítulo 5), decidiu-se implementar o A C-F OVE em sua forma genérica, independente de uma lógica de descrição específica. Um algoritmo de inferência aproximada já existe para o Credal $\mathcal{A L C}$ (POLASTRO; COZMAN, 2008), entretanto não existe nenhum algoritmo de inferência exata em primeira ordem que já tenha sido aplicado à lógica. De fato, não existia, até a data da proposta deste trabalho, uma implementação publicamente disponível do algoritmo AC-F OVE.

Além de fornecer uma implementação do algoritmo, este trabalho visa complementar alguns pontos da teoria proposta por Kisynski (2010):

- Formalização matemática de fórmulas para o cálculo de custos do algoritmo;

- Correção da teoria para eliminação de variáveis com agregação.

Uma vez terminada a implementação, deseja-se testar o desempenho do algoritmo em modelos propostos na literatura para verificar sua escalabilidade. O desempenho do algoritmo AC-F OVE será comparado com os algoritmos C-FOVE e VE. Estes dois últimos algoritmos foram implementados como passos intermediários para obter o A C-F OVE, uma vez que ambos usam um subconjunto das operações do A C-F OVE.

\subsection{Organização do Texto}

O restante do trabalho é organizado como se segue. No Capítulo 2 são revisados os conceitos de Redes Bayesianas (Seção 2.1) e inferência exata proposicional (Seção 2.2).

O algoritmo A C-F OVE é bastante complexo e apresenta muitas sutilezas. Os conceitos e termos fundamentais atrelados ao algoritmo são apresentados na Seção 3.1. Em seguida são detalhadas as funções elementares do algoritmo (Seção 3.2), que por sua vez são agrupadas em macro-operações (Seção 3.3) para compor o algoritmo principal (Seção 3.4).

O Capítulo 4 é dedicado à metodologia utilizada na estruturação do software (Seção 4.1) e às etapas estabelecidas para o seu desenvolvimento (Seção 4.2). Na Seção 4.3 apresenta-se um guia de utilização do software criado.

Embora Kisynski (2010) tenha consolidado toda a teoria do algoritmo AC-FOVE em sua tese de doutorado, o trabalho de implementação revelou a existência de pontos 
não esclarecidos ou faltantes da teoria. No Capítulo 5, realiza-se uma consolidação das contribuições deste trabalho à teoria utilizada pelo algoritmo A C-F OVE.

No Capítulo 6, são detalhados os experimentos realizados com a implementação do algoritmo.

Finalmente, no Capítulo 7 tecem-se as considerações finais e as expectativas de continuação do trabalho. 


\section{Redes Bayesianas}

Neste capítulo serão introduzidos a notação e os conceitos utilizados ao longo do texto. Apresentam-se a teoria básica de Redes Bayesianas (Seção 2.1) e o algoritmo de eliminação de variáveis (Seção 2.2), utilizado para realizar inferências em redes Bayesianas. O algoritmo de eliminação de variáveis é a base para o algoritmo AC-F OVE.

\subsection{Redes Bayesianas}

Redes Bayesianas foram propostas por Pearl (1988) como modelos de representação de relações de dependência entre um conjunto de variáveis aleatórias. Ao longo dos anos este modelo se tornou bastante popular e encontrou aplicações em diversas áreas.

Definição 1. Uma Rede Bayesiana (BN) sobre um conjunto de variáveis aleatórias $\left\{X_{1}\right.$, $\left.X_{2}, \ldots, X_{n}\right\}$ consiste em um grafo acíclico dirigido em que cada nó representa uma variável aleatória. Os arcos que ligam os nós representam a relação de dependência entre eles. Cada nó do grafo está associado a uma distribuição de probabilidades condicionais $\left\{P\left(X_{i} \mid \operatorname{pais}\left(X_{i}\right)\right) \mid i=1,2, \ldots, n\right\}$ em que pais $\left(X_{i}\right)$ denota os nós no grafo cujos arcos apontam para o nó $X_{i}$. Uma Rede Bayesiana representa a distribuição de probabilidade conjunta das variáveis aleatórias $X_{1}, X_{2}, \ldots, X_{n}$ :

$$
P\left(X_{1}, X_{2}, \ldots, X_{n}\right)=\prod_{i=1}^{n} P\left(X_{i} \mid \operatorname{pais}\left(X_{i}\right)\right)
$$

Exemplo 1. A Figura 1 (CHARNIAK, 1991) apresenta uma Rede Bayesiana simples com cinco variáveis aleatórias:

- family_out indica se a família está fora de casa;

- light_on indica se a luz da casa está acesa;

- dog_out indica se o cachorro da família está no quintal; 
- bowel_problem indica se o cachorro está com problemas intestinais; e

- hear_bark indica se é possível ouvir os latidos do cachorro.

Assume-se que todas as variáveis aleatórias são booleanas, isto é, possuem dominio \{falso, verdadeiro\}. Associadas à esta Rede Bayesiana tem-se as probabilidades condicionais indicadas. Note que na Figura 1 o conjunto de probabilidades explicitado é suficiente

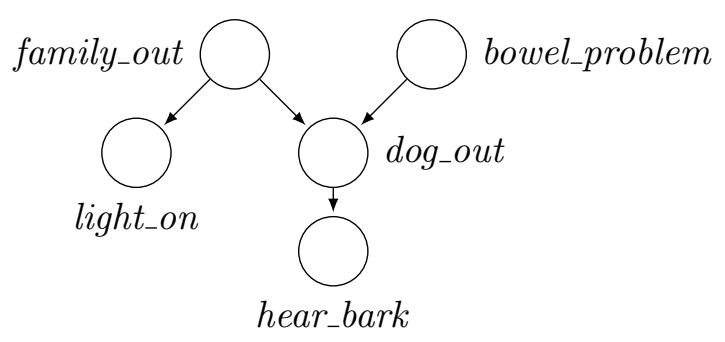

$$
\begin{aligned}
& P(\text { family_out })=0.5 \quad P(\text { dog_out } \mid \text { family_out }, \text { bowel_problem })=0.8 \\
& P(\text { bowel_problem })=0.5 \quad P(\text { dog_out } \neg \text { family_out }, \text { bowel_problem })=0.1 \\
& P(\text { light_on } \mid \text { family_out })=0.6 \quad P(\text { dog_out } \text { family_out }, \neg \text { bowel_problem })=0.1 \\
& P(\text { light_on } \mid \neg \text { family_out })=0.05 \quad P(\text { dog_out } \mid \neg \text { family_out }, \neg \text { bowel_problem })=0.7 \\
& P\left(\text { hear_bark } \mid d_{\text {dog_out }}\right)=0.6 \quad P(\text { hear_bark } \mid \neg \text { dog_out })=0.3
\end{aligned}
$$

Figura 1: Rede Bayesiana com 5 variáveis aleatórias.

para determinar a distribuição de probabilidade representada pela rede. Por exemplo, $P($ family_out $)=0.5$ implica em $P(\neg$ family_out $)=0.5$ e $P($ light_on $\mid$ family_out $)=0.6$ implica em $P(\neg$ light_on $\mid$ family_out $)=0.4$.

Uma das vantagens das Redes Bayesianas é a compacidade da representação. Para uma rede com $n$ variáveis aleatórias binárias, é necessário especificar $2 n$ valores de probabilidade, enquanto que a especificação de todas as combinações sem a noção de dependência entre as variáveis necessitaria de $2^{n}-1$ valores.

\subsection{Inferência em Redes Bayesianas}

Em uma Rede Bayesiana, comumente deseja-se computar a distribuição de probabilidade a posteriori de um conjunto de variáveis aleatórias dada alguma evidência. Um algoritmo clássico para a resolução deste problema é a eliminação de variáveis (VE) (ZHANG; POOLE, 1994). Este algoritmo utiliza estruturas denominadas fatores, que são utilizadas para representar a entrada do problema e armazenar os resultados intermediários e a solução final. 


\subsubsection{Fatores}

Para toda variável aleatória $X$, denota-se por $\mathcal{D}(X)$ o domínio de $X$ (KISYNSKI, 2010). Um fator nas variáveis aleatórias $X_{1}, X_{2}, \ldots, X_{n}$ é uma representação de uma função $\mathcal{F}: \mathcal{D}\left(X_{1}\right) \times \mathcal{D}\left(X_{2}\right), \times \cdots \times \mathcal{D}\left(X_{n}\right) \rightarrow \mathbb{R}($ KISYNSKI, 2010).

Exemplo 2. Considere a Rede Bayesiana do Exemplo 1. A distribuição de probabilidade condicional do nó dog_out pode ser representada pelo seguinte fator:

\begin{tabular}{cccc}
\hline family_out & bowel_problem & dog_out & VALOR \\
\hline F & F & F & 0.70 \\
F & F & V & 0.30 \\
F & V & F & 0.03 \\
F & V & V & 0.97 \\
V & F & F & 0.10 \\
V & F & V & 0.90 \\
V & V & F & 0.01 \\
V & V & V & 0.99 \\
\hline
\end{tabular}

Existem duas operações que podem ser aplicadas a fatores: multiplicação e eliminação (sum out).

Definição 2. Seja $\mathcal{F}_{1}$ um fator nas variáveis aleatórias $X_{1}, \ldots, X_{i}, Y_{1}, \ldots, Y_{j}$ e seja $\mathcal{F}_{2}$ um fator nas variáveis aleatórias $Y_{1}, \ldots, Y_{j}, Z_{1}, \ldots, Z_{k}$, com os conjuntos $\left\{X_{1}, \ldots, X_{i}\right\}$, $\left\{Y_{1}, \ldots, Y_{j}\right\},\left\{Z_{1}, \ldots, Z_{k}\right\}$ disjuntos dois a dois. O produto $\mathcal{F}_{1} \odot \mathcal{F}_{2}$ é um fator nas variáveis $X_{1}, \ldots, X_{i}, Y_{1}, \ldots, Y_{j}, Z_{1}, \ldots, Z_{k}$ (POOLE; MACKWORTH, 2010) definido por:

$$
\begin{aligned}
\left(\mathcal{F}_{1} \odot \mathcal{F}_{2}\right)\left(X_{1}, \ldots, X_{i}, Y_{1}, \ldots, Y_{j}, Z_{1}, \ldots, Z_{k}\right) & = \\
& \mathcal{F}_{1}\left(X_{1}, \ldots, X_{i}, Y_{1}, \ldots, Y_{j}\right) \times \mathcal{F}_{2}\left(Y_{1}, \ldots, Y_{j}, Z_{1}, \ldots, Z_{k}\right)
\end{aligned}
$$

Exemplo 3. Considere os fatores $\mathcal{F}_{1}$ e $\mathcal{F}_{2}$ representados a seguir:

$$
\mathcal{F}_{1}=\begin{array}{ccc}
\mathrm{A} & \mathrm{B} & \\
\hline \mathrm{F} & \mathrm{F} & 0.1 \\
\mathrm{~F} & \mathrm{~V} & 0.2 \\
\mathrm{~V} & \mathrm{~F} & 0.3 \\
\mathrm{~V} & \mathrm{~V} & 0.5 \\
\hline
\end{array}
$$

$\begin{array}{lll}\text { F } & \text { F } & 0.1\end{array}$

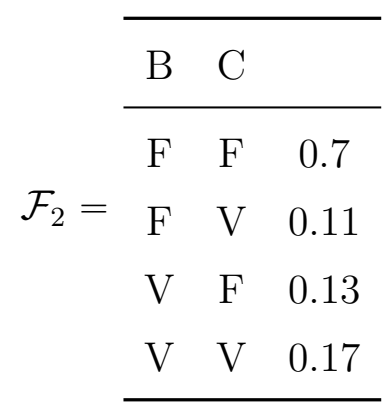

Para determinar o produto $\mathcal{F}_{1} \odot \mathcal{F}_{2}$, monta-se um fator que contém a união de todas as variáveis aleatórias dos fatores $\mathcal{F}_{1}$ e $\mathcal{F}_{2}$. Em seguida, para cada linha desse fator, 
multiplica-se o valor determinado pelo subconjunto das variáveis aleatórias em $\mathcal{F}_{1}$ pelo valor determinado pelo subconjunto das variáveis aleatórias em $\mathcal{F}_{2}$. Por exemplo, $\left(\mathcal{F}_{1} \odot\right.$ $\left.\mathcal{F}_{2}\right)(F, F, F)=\mathcal{F}_{1}(F, F) \times \mathcal{F}_{2}(F, F)$. O produto $\mathcal{F}_{1} \odot \mathcal{F}_{2}$ é dado por:

\begin{tabular}{ccccc}
\cline { 2 - 4 } A & B & C & \\
\cline { 2 - 4 } & F & F & F & 0.07 \\
F & F & V & 0.011 \\
$\mathcal{F}_{1} \odot \mathcal{F}_{2}=$ & F & V & F & 0.026 \\
F & V & V & 0.034 \\
V & F & F & 0.21 \\
V & F & V & 0.033 \\
V & V & F & 0.065 \\
V & V & V & 0.085 \\
\hline
\end{tabular}

Definição 3. Seja $\mathcal{F}$ um fator nas variáveis aleatórias $X_{1}, \ldots, X_{i}, \ldots, X_{j}$. Eliminar a variável aleatória $X_{i}$ do fator $\mathcal{F}$ resulta em um fator nas variáveis aleatórias $X_{1}, \ldots$, $X_{i-1}, X_{i+1}, \ldots, X_{j}$ (POOLE; MACKWORTH, 2010), denotado por $\sum_{X_{i}} \mathcal{F}$ e definido por:

$$
\left(\sum_{X_{i}} \mathcal{F}\right)\left(X_{1}, \ldots, X_{i-1}, X_{i+1}, \ldots, X_{j}\right)=\sum_{k \in \mathcal{D}\left(X_{i}\right)} \mathcal{F}\left(X_{1}, \ldots, X_{i-1}, X_{i}=k, X_{i+1}, \ldots, X_{j}\right)
$$

Exemplo 4. Considere o fator $\mathcal{F}$ representado a seguir:

$$
\mathcal{F}=\begin{array}{ccc}
\hline \mathrm{A} & \mathrm{B} & \\
\hline \mathrm{F} & \mathrm{F} & 0.1 \\
\mathrm{~F} & \mathrm{~V} & 0.9 \\
\mathrm{~V} & \mathrm{~F} & 0.3 \\
\mathrm{~V} & \mathrm{~V} & 0.7 \\
\hline
\end{array}
$$

A eliminação da variável aleatória $A$ de $\mathcal{F}$ resulta em

$$
\Sigma_{A} \mathcal{F}=\begin{array}{ll}
\frac{\mathrm{B}}{\mathrm{F}} & 0.4 \\
\mathrm{~V} & 1.6
\end{array}
$$




\subsubsection{Algoritmo de Eliminação de Variáveis}

Nesta seção apresenta-se o algoritmo de eliminação de variáveis (vE) para realizar inferências em Redes Bayesianas (ZHANG; POOLE, 1994). O algoritmo basicamente utiliza a operação de eliminação sucessivamente de modo a obter a probabilidade marginal $a$ posteriori desejada.

Considere uma rede Bayesiana no conjunto de variáveis aleatórias $V=\left\{X_{1}, X_{2}, \ldots\right.$, $\left.X_{n}\right\}$. Para cada variável aleatória $X_{i}$ em $V$, representa-se a distribuição de probabilidade condicional $P\left(X_{i} \mid\right.$ pais $\left.\left(X_{i}\right)\right)$ como um fator $\mathcal{F}_{X_{i}}$ no conjunto de variáveis aleatórias $\left\{X_{i}\right\} \cup$ pais $\left(X_{i}\right)$. Considere ainda que sejam dados:

- Um conjunto de observações de variáveis aleatórias $E \subset V$. O conjunto $E$ recebe o nome de evidência; e

- Uma variável aleatória de consulta $q \in V \backslash E$.

Seja $V \backslash\{E \cup\{q\}\}=\left\{X_{s_{1}}, X_{s_{2}}, \ldots, X_{s_{m}}\right\}$. Deseja-se calcular a probabilidade marginal em $q$ e $E$ :

$$
\sum_{X_{s_{1}}} \sum_{X_{s_{2}}} \cdots \sum_{X_{s_{m}}} \mathcal{F}_{X_{1}} \odot \mathcal{F}_{X_{2}} \odot \cdots \odot \mathcal{F}_{X_{n}}
$$

Geralmente, o cálculo do produto $\mathcal{F}_{X_{1}} \odot \mathcal{F}_{X_{2}} \odot \cdots \odot \mathcal{F}_{X_{n}}$ é computacionalmente custoso. Faz-se então uma ordenação das somatórias de acordo com uma ordem de eliminação $\rho$ :

$$
\sum_{X_{\rho(m)}} \ldots \sum_{X_{\rho(2)}} \sum_{X_{\rho(1)}} \mathcal{F}_{X_{1}} \odot \mathcal{F}_{X_{2}} \odot \cdots \odot \mathcal{F}_{X_{n}}
$$

A seguir, utiliza-se a propriedade distributiva, colocando os fatores que não são funções de $X_{\rho(1)}$ fora da somatória $\sum_{X_{\rho(1)}}$. Os fatores remanescentes são então multiplicados e, em seguida, elimina-se $X_{\rho(1)}$ do produto. Este processo se repete para as somatórias de $\sum_{X_{\rho}(2)}$ até $\sum_{X_{\rho}(m)}$. O Algoritmo 1 ilustra o pseudocódigo para VE. 


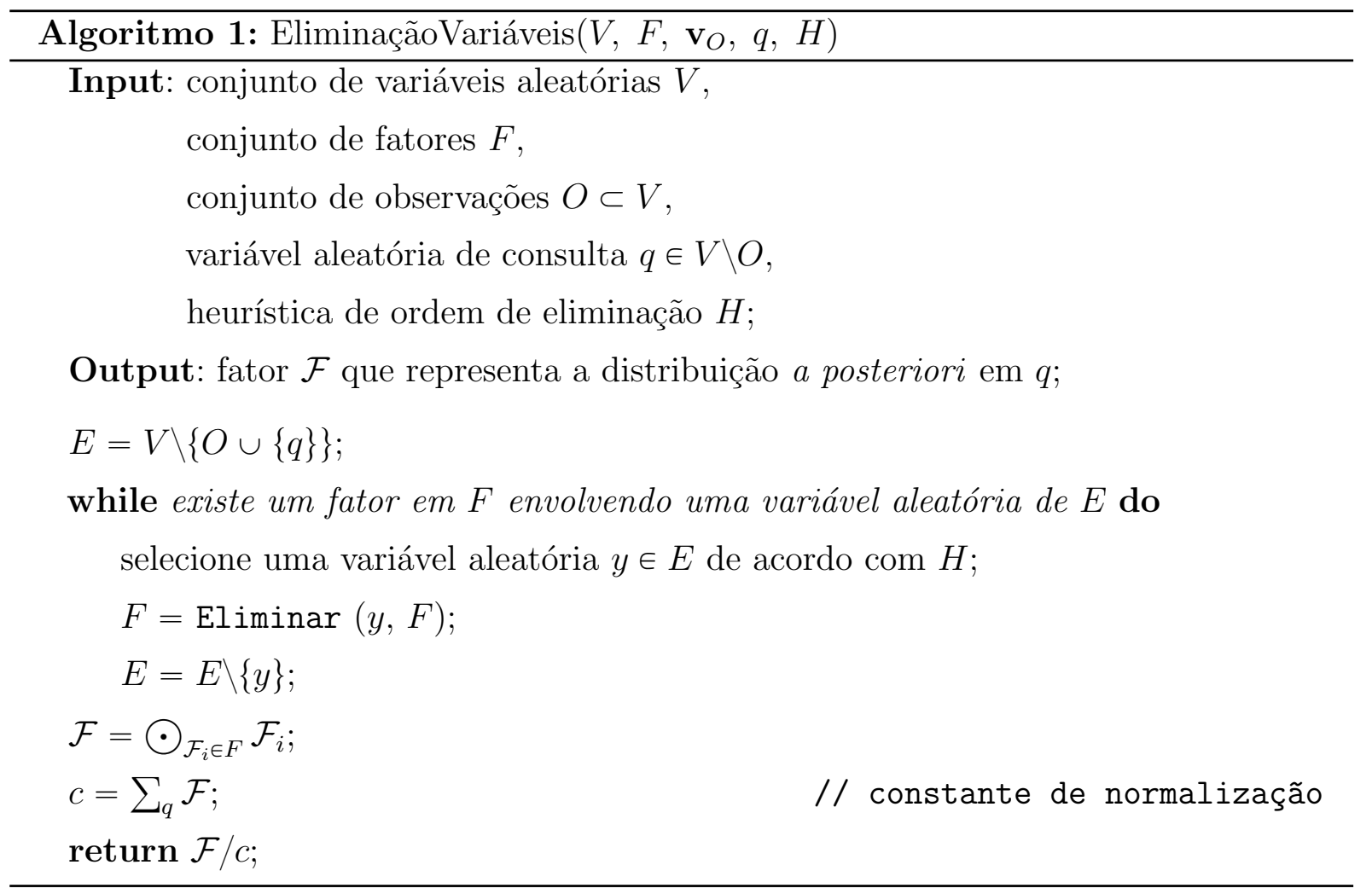

Procedimento Eliminar $(y, F)$

Input: variável aleatória a ser eliminada $y$, conjunto de fatores $F$;

Output: conjunto de fatores $F$ com $y$ eliminado;

$F_{y}=$ conjunto de fatores em $F$ que contêm $y$;

$F_{-y}=F \backslash F_{y}$;

return $F_{-y} \cup\left\{\sum_{y} \bigodot_{\mathcal{F}_{i} \in F_{y}} \mathcal{F}_{i}\right\}$; 


\section{O Algoritmo AC-FOVE}

O AC-FOVE é um algoritmo proposto por Kisynski e Poole (2009) para realizar inferências sobre modelos probabilísticos em primeira ordem. Na Seção 3.1 definem-se os principais conceitos que serão utilizados nas demais seções. A Seção 3.2 detalha as operações elementares do algoritmo, que por sua vez são agregadas em macro-operações, detalhadas na Seção 3.3. Finalmente, a Seção 3.4 consolida o funcionamento do algoritmo.

\subsection{Conceitos e definições}

Nesta seção serão detalhados os conceitos e as definições que serão usadas ao longo do texto.

Uma população é um conjunto de indivíduos, e um parâmetro é uma variável lógica atrelada a uma população. Os termos "parâmetro" e "variável lógica" serão utilizados de maneira intercambiável neste trabalho. Dado um parâmetro $X$, representa-se a sua população por $\mathcal{D}(X)$. Um termo é uma variável lógica ou uma constante.

\subsubsection{Variáveis aleatórias parametrizadas}

Uma variável aleatória parametrizada é representada na forma $f\left(t_{1}, \ldots, t_{k}\right)$, em que $f$ é um funtor e $t_{1}, \ldots, t_{k}$ são termos. Cada funtor $f$ pode assumir um conjunto de valores denominado domínio e denotado por RANGE $(f)$. O conjunto dos parâmetros de uma variável aleatória parametrizada $f\left(t_{1}, \ldots, t_{k}\right)$ é denotado por PARA M $(f)$.

Uma substituição em um conjunto de variáveis lógicas distintas $\left\{X_{1}, \ldots, X_{k}\right\}$ é representada na forma $\theta=\left\{X_{1} / t_{1}, \ldots, X_{k} / t_{k}\right\}$, em que cada $X_{i}$ é uma variável lógica e cada $t_{i}$ é uma variável lógica ou uma constante. Ao aplicar a substituição $\theta=\left\{X_{1} / t_{i 1}, \ldots, X_{m} / t_{i m}\right\}$ a uma variável aleatória parametrizada $f\left(t_{1}, \ldots, t_{k}\right)$, obtém-se uma variável aleatória parametrizada com cada ocorrência de $X_{j}$ substituída pelo respectivo termo $t_{i j}$. A nova 
variável aleatória parametrizada é representada por $f\left(t_{1}, \ldots, t_{k}\right)[\theta]$ e é denominada uma instância de $f\left(t_{1}, \ldots, t_{k}\right)$.

Uma substituição elementar é uma substituição $\left\{X_{1} / t_{1}, \ldots, X_{k} / t_{k}\right\}$ em que cada $t_{i}$ é uma constante. Se uma substituição elementar é aplicada a todas as variáveis lógicas de uma variável aleatória parametrizada $f\left(t_{1}, \ldots, t_{k}\right)$, obtém-se uma instância elementar de $f\left(t_{1}, \ldots, t_{k}\right)$. Proposicionalizar uma variável lógica consiste em realizar todas as possíveis substituições elementares para esta variável.

Uma variável aleatória parametrizada $f\left(t_{1}, \ldots, t_{k}\right)$ representa um conjunto de variáveis aleatórias. Este conjunto é obtido realizando-se todas as possíveis substituições elementares nos parâmetros de $f$. Este conjunto é representado por $\operatorname{RV}(f)$.

Exemplo 5. Considere uma variável lógica $P$ atrelada à população de todas as $n$ pessoas em uma universidade: $\mathcal{D}(P)=\left\{p_{1}, \ldots, p_{n}\right\}$. Seja estudante $(P)$ uma variável aleatória parametrizada, em que estudante é um functor com domínio $\{\mathrm{V}, \mathrm{F}\}$. Tem-se $\mathrm{RANGE}($ estudante $)=$ $\{\mathrm{V}, \mathrm{F}\}$ e PARA $\mathrm{P}($ estudante $(P))=\{P\}$.

A variável aleatória parametrizada estudante $(P)$ representa um conjunto de $n$ variáveis aleatórias, uma para cada substituição $\left\{P / p_{1}, \ldots, P / p_{n}\right\}$. A proposicionalização da variável lógica $P$ resulta no conjunto de variáveis aleatórias $\left\{\right.$ estudante $\left(p_{1}\right), \ldots$, estudante $\left.\left(p_{n}\right)\right\}$.

Uma restrição de desigualdade tem a forma $X \neq t$, em que $X$ é uma variável lógica e $t$ é um termo. Restrições tem a finalidade de limitar o conjunto de substituições que podem ser aplicadas a uma variável lógica. Neste trabalho, um conjunto de restrições será representado por $\mathcal{C}$. Dados uma variável aleatória parametrizada $f\left(t_{1}, \ldots, t_{k}\right)$, um conjunto de restrições $\mathcal{C}$ e uma instância elementar $f\left(t_{1}, \ldots, t_{k}\right)[\theta]$, diz-se que a instância elementar $f\left(t_{1}, \ldots, t_{k}\right)[\theta]$ satisfaz as restrições em $\mathcal{C}$ se a substituição $\theta$ satisfaz as restrições em $\mathcal{C}$. O conjunto das variáveis aleatórias representadas por $f\left(t_{1}, \ldots, t_{k}\right)$ que satisfazem as restrições em $\mathcal{C}$ é denotado por $\operatorname{RV}(f): \mathcal{C}$.

Seja $f_{1}$ uma variável aleatória parametrizada e $\mathcal{C}_{1}$ um conjunto de restrições de desigualdade envolvendo variáveis lógicas de $f_{1}$. Seja $f_{2}$ uma variável aleatória parametrizada e $\mathcal{C}_{2}$ um conjunto de restrições de desigualdade envolvendo variáveis lógicas de $f_{2}$. Neste trabalho, diz-se que $f_{1}$ é equivalente a $f_{2}$ se o conjunto de variáveis aleatórias representadas por $f_{1}$ é igual ao conjunto de variáveis aleatórias representadas por $f_{2}$, isto é, $\operatorname{RV}\left(f_{1}\right): \mathcal{C}_{1}=\operatorname{RV}\left(f_{2}\right): \mathcal{C}_{2}$ 


\subsubsection{Fórmulas de contagem}

Até o momento, uma atribuição de valores a uma variável aleatória parametrizada pode ser usada para especificar valores de indivíduos específicos ou de todos os indivíduos de uma população. Em algumas situações, é útil poder dizer que um certo número de indivíduos assume um determinado valor, sem precisar especificar quais deles assumem esse valor. Isto permitiria diminuir o tamanho da representação de um fator. Por exemplo, suponha que uma variável aleatória parametrizada booleana $f(X)$ represente três variáveis aleatórias: $f\left(x_{1}\right), f\left(x_{2}\right), f\left(x_{3}\right)$. Existem 8 combinações de valores possíveis que estas variáveis podem assumir. Entretanto, como será visto adiante neste capítulo, nem sempre é essencial enumerar todas as possíveis valorações que estas variáveis assumem, sendo suficiente saber apenas quantas assumem o valor verdadeiro e quantas são falsas (quando se trata de variáveis aleatórias booleanas). Neste caso, existem 4 valores possíveis: 3 são verdadeiras e nenhuma é falsa, 2 são verdadeiras e 1 é falsa, 1 é verdadeira e 2 são falsas, nenhuma é verdadeira e 3 são falsas.

Fórmulas de contagem (MILCH et al., 2008) são variáveis aleatórias parametrizadas que representam uma contagem de valores de uma variável aleatória parametrizada. A contagem é representada por meio de histogramas de valores.

Definição 4. Um histograma é uma ênupla $\left(x_{1}, \ldots, x_{n}\right)$ em que cada $x_{i}$ é um inteiro não negativo.

Ao longo do texto, uma representação mais prolixa para histogramas também será utilizada: $\left(\#_{\alpha_{1}}=x_{1}, \ldots, \#_{\alpha_{n}}=x_{n}\right.$ ), em que $\alpha_{i} \in$ é um elemento do domínio de tamanho $n$ de uma variável aleatória parametrizada $f$, isto é, $\operatorname{R~ANGE}(f)=\left\{\alpha_{1}, \ldots, \alpha_{n}\right\}$. Dado um histograma $h=\left(\#_{\alpha_{1}}=x_{1}, \ldots, \#_{\alpha_{n}}=x_{n}\right)$, o valor do i-ésimo elemento $\left(x_{i}\right)$ será denotado por $h\left(\alpha_{i}\right)$.

Exemplo 6. Considere uma variável aleatória parametrizada resultado $(D)$ que representa a face voltada para cima quando um dado D é jogado. Tem-se R ANGE(resultado) = $\{1,2,3,4,5,6\}$ e $\mathcal{D}(D)=\left\{d_{1}, \ldots, d_{10}\right\}$. Então o histograma $\left(\#_{1}=2, \#_{2}=4, \#_{3}=\right.$ $1, \#_{4}=0, \#_{5}=2, \#_{6}=1$ ) representa um possível resultado obtido num experimento em que os dados $d_{1}, \ldots, d_{10}$ são arremessados ao mesmo tempo. O mesmo histograma pode ser representado por $(2,4,1,0,2,1)$ se a ordem dos elementos de $\mathrm{R}$ A NGE(resultado) for previamente fixada.

Uma fórmula de contagem tem a forma $\#_{A: \mathcal{C}}[f]$, em que $A$ é uma variável lógica, $\mathcal{C}$ é um conjunto de restrições de desigualdade envolvendo a variável $A$ e $f$ é uma variável 
aleatória parametrizada contendo $A$. Uma fórmula de contagem $\gamma=\#_{A: \mathcal{C}}[f]$ tem as seguintes propriedades:

- $\operatorname{RV}(\gamma)=\operatorname{RV}(f): \mathcal{C}$

- PARAm $(\gamma)=\operatorname{ParAM}(f) \backslash\{A\}$

O conjunto RANGE $(\gamma)$ é formado por todos os possíveis histogramas cuja soma de seus elementos seja igual a $|\mathcal{D}(A): \mathcal{C}|$. Pode-se $\operatorname{mostrar}^{1}$ que o tamanho de $\operatorname{RANGE}(\gamma)$ é dado pelo coeficiente binomial $\left(\begin{array}{c}|\operatorname{RangE}(f)|+|\mathcal{D}(X): \mathcal{C}|-1 \\ |\operatorname{RanGE}(f)|-1\end{array}\right)$ (FELLER, 1968).

Exemplo 7. Seja $f(A, B)$ uma variável aleatória parametrizada com $\mathrm{RANGE}=\{\mathrm{F}$, V\}. Considere que a população atrelada à variável lógica A seja dada pelo conjunto $\mathcal{D}(A)=\left\{a_{1}, \ldots, a_{5}\right\}$ e a população atrelada à variável lógica $B$ seja $\mathcal{D}(B)=\left\{b_{1}, b_{2}\right\}$.

Seja $\mathcal{C}=\left\{A \neq a_{1}, A \neq a_{2}, A \neq a_{5}\right\}$ um conjunto de restrições de desigualdade. Tem-se $\mathcal{D}(A): \mathcal{C}=\left\{a_{3}, a_{4}\right\} . A$ fórmula de contagem $\gamma=\#_{A: \mathcal{C}}[f]$ terá as seguintes propriedades:

- $\operatorname{RV}(\gamma)=\left\{f\left(a_{3}, b_{1}\right), f\left(a_{3}, b_{2}\right), f\left(a_{4}, b_{1}\right), f\left(a_{4}, b_{2}\right)\right\}$

- $\operatorname{PARAM}(\gamma)=\{B\}$

- $\operatorname{RANGE}(\gamma)=\left\{\left(\#_{\mathrm{F}}=2, \#_{\mathrm{V}}=0\right),\left(\#_{\mathrm{F}}=1, \#_{\mathrm{V}}=1\right),\left(\#_{\mathrm{F}}=0, \#_{\mathrm{V}}=2\right)\right\}$

Fórmulas de contagem são úteis quando o valor de um fator depende somente do número de variáveis aleatórias que assumem um determinado valor. Suponha por exemplo um ônibus que não faz paradas intermediárias equipado com uma catraca. Seja $P$ a variável lógica atrelada ao conjunto de indivíduos que frequentam o ônibus: $\left\{p_{1}, \ldots, p_{n}\right\}$. Considere ainda duas variáveis aleatórias parametrizadas booleanas: passou $(P)$ (que indica que a pessoa passou pela catraca) e vazio() (que indica que o ônibus está vazio). A variável vazio() assume valor $\mathrm{V}$ quando o número de pessoas que passaram pela catraca é zero.

A dependência entre as variáveis vazio() e passou $(P)$ pode ser traduzida por um fator nas variáveis vazio () , passou $\left(p_{1}\right), \ldots$ passou $\left(p_{n}\right)$. Entretanto, a representação do fator terá um tamanho exponencial no número de pessoas que frequentam o ônibus $(n)$, o que não é conveniente do ponto de vista computacional.

Outra maneira de representar esta dependência é utilizar a fórmula de contagem $\#_{P}[$ passou $]$. Os possíveis valores desta fórmula de contagem são histogramas que contam a quantidade de pessoas que passaram e que não passaram pela catraca do ônibus.

\footnotetext{
${ }^{1}$ Note que o problema é análogo a contar o número de maneiras de distribuir $r$ bolas indistinguíveis em $n$ urnas.
} 
Os valores possíveis de passou $(P)$ são $\{\mathrm{F}, \mathrm{V}\}$, então cada histograma será uma tupla de dois elementos, um com a contagem de variáveis que assumem o valor $\mathrm{F}$ e outro com a contagem de variáveis que assumem o valor $\mathrm{V}$. A soma de todas as contagens de um histograma deve ser o tamanho da população de $P$. Assim, se $n=10$, haverá 11 histogramas possíveis: $(0,10),(1,9), \ldots,(10,0)$.

Suponha agora que $n=3$. Então

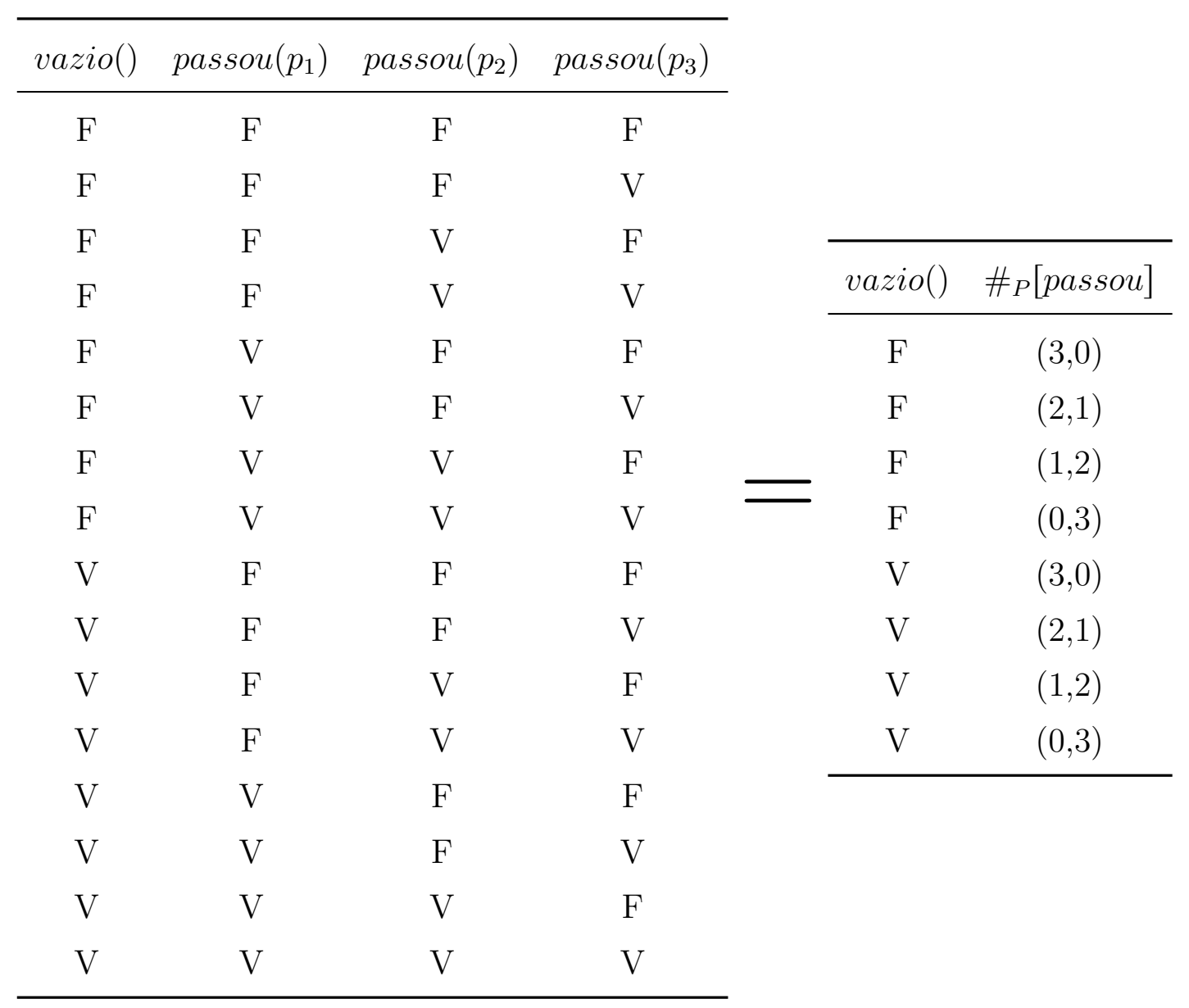

Note que o fator com a fórmula de contagem tem um tamanho que depende linearmente do tamanho da população de $P$.

\subsubsection{Parfactors}

Para realizar uma eliminação de variáveis em primeira ordem, é necessário criar estruturas de dados que sejam análogas aos fatores em variáveis aleatórias. Poole (2003) propôs os parfactors como estruturas com a função de representar distribuições de probabilidade em modelos direcionados de primeira ordem.

Definição 5. Um parfactor (POOLE, 2003) é uma tripla $\langle\mathcal{C}, \mathcal{V}, \mathcal{F}\rangle$, em que: 
- $\mathcal{C}$ é um conjunto de restrições de desigualdade envolvendo duas variáveis lógicas ou uma variável lógica e uma constante;

- $\mathcal{V}$ é um conjunto de variáveis lógicas aleatórias parametrizadas. Para todo par de variáveis aleatórias parametrizadas $f$ e $h$ de $\mathcal{V}$, tem-se

$$
\operatorname{RV}(f): \mathcal{C} \cap \operatorname{RV}(h): \mathcal{C}=\varnothing
$$

- $\mathcal{F}$ é um fator que representa uma função $f: \times_{f \in \mathcal{V}} \mathrm{RANGE}(f) \rightarrow \mathbb{R}$.

A condição (3.1.1) garante que toda substituição elementar nas variáveis de $\mathcal{V}$ resulta em conjuntos de variáveis aleatórias de mesmo tamanho.

Exemplo 8. Neste exemplo mostra-se a necessidade da condição (3.1.1). Considere um parfactor $g=\langle\mathcal{C}, \mathcal{V}, \mathcal{F}\rangle$ com $\mathcal{C}=\varnothing$ e $\mathcal{V}=\{f(A), f(B)\}$. Suponha que $\mathcal{D}(A)=\mathcal{D}(B)=$ $\left\{x_{1}, x_{2}\right\}$.

Note que o parfactor g não obedece à condição (3.1.1). Suponha que sejam aplicadas duas substituições diferentes nas variáveis deste parfactor: $\theta_{1}=\left\{A / x_{1}, B / x_{2}\right\}$ e $\theta_{2}=$ $\left\{A / x_{1}, B / x_{1}\right\}$.

No primeiro caso, o conjunto de variáveis aleatórias parametrizadas $\mathcal{V}$ se torna $\mathcal{V}\left[\theta_{1}\right]=$ $\left\{f\left(x_{1}\right), f\left(x_{2}\right)\right\}$. Já no segundo caso, obtém-se $\mathcal{V}\left[\theta_{2}\right]=\left\{f\left(x_{1}\right)\right\}$. Veja que o tamanho do conjunto $\mathcal{V}$ varia dependendo da substituição que é feita.

A importância da condição (3.1.1) ficará evidente na seção 3.2.7.

Assume-se neste trabalho que todas as restrições do conjunto $\mathcal{C}$ são consistentes, isto é:

- Toda variável lógica presente em uma restrição de $\mathcal{C}$ existe em alguma variável aleatória parametrizada do conjunto $\mathcal{V}$;

- Se uma restrição envolve uma variável lógica e uma constante, então a constante pertence à população desta variável lógica;

- Se uma restrição envolve duas variáveis lógicas, então as duas variáveis estão atreladas à mesma população.

O conjunto das variáveis lógicas presentes nas variáveis aleatórias parametrizadas de um parfactor $g$ é denotado por $\operatorname{LV}(g)$. 
Um parfactor $g=\langle\mathcal{C}, \mathcal{V}, \mathcal{F}\rangle$ representa um conjunto de fatores. Este conjunto é obtido através da aplicação de todas as possíveis substituições elementares às variáveis lógicas presentes em $\mathcal{V}$ e que satisfazem as restrições em $\mathcal{C}$. O tamanho deste conjunto é denotado por $|g|$ (KISYNSKI, 2010):

$$
|g|=\prod_{A \in \operatorname{LV}(g)}|\mathcal{D}(A): \mathcal{C}|
$$

Exemplo 9. Seja $\mathcal{V}=\{f(A), h(B)\}, \mathcal{D}(A)=\left\{a_{1}, a_{2}\right\}, \mathcal{D}(B)=\left\{b_{1}, b_{2}, b_{3}, b_{4}\right\}$ e $\mathcal{C}=$ $\left\{A \neq a_{1}, B \neq b_{1}, B \neq b_{2}\right\}$. O parfactor $\langle\mathcal{C}, \mathcal{V}, \mathcal{F}\rangle$ representa $(2-1) \cdot(4-2)=2$ fatores: $\mathcal{F}\left(f\left(a_{2}\right), h\left(b_{3}\right)\right)$ e $\mathcal{F}\left(f\left(a_{2}\right), h\left(b_{4}\right)\right)$.

Exemplo 10. Considere uma versão de primeira ordem da rede Sprinkler proposta por Murphy (1998), representada na Figura 2.

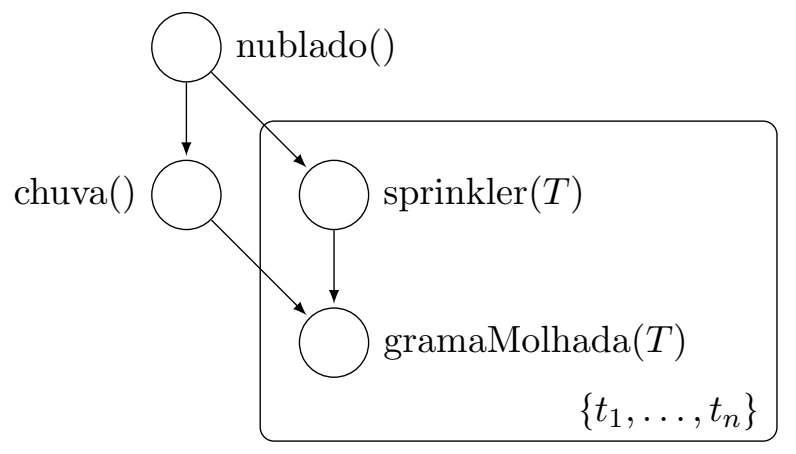

Figura 2: Versão de primeira ordem da rede Sprinkler (MURPHY, 1998)

A rede consiste em quatro variáveis aleatórias parametrizadas: nublado(), chuva(), sprinkler $(T)$, gramaMolhada $(T)$. Todas tem domínio booleano, isto é, R A N GE (nublado) $=$ $\mathrm{RANGE}($ chuva $)=\mathrm{RANGE}($ sprinkler $)=\mathrm{RANGE}($ gramaMolhada $)=\{F, V\}$. A variável lógica $T$ está atrelada a um conjunto de terrenos de uma região: $\mathcal{D}(T)=\left\{t_{1}, \ldots, t_{n}\right\}$. A rede Bayesiana correspondente, obtida proposicionalizando-se todas as variáveis lógicas, é ilustrada na Figura 3.

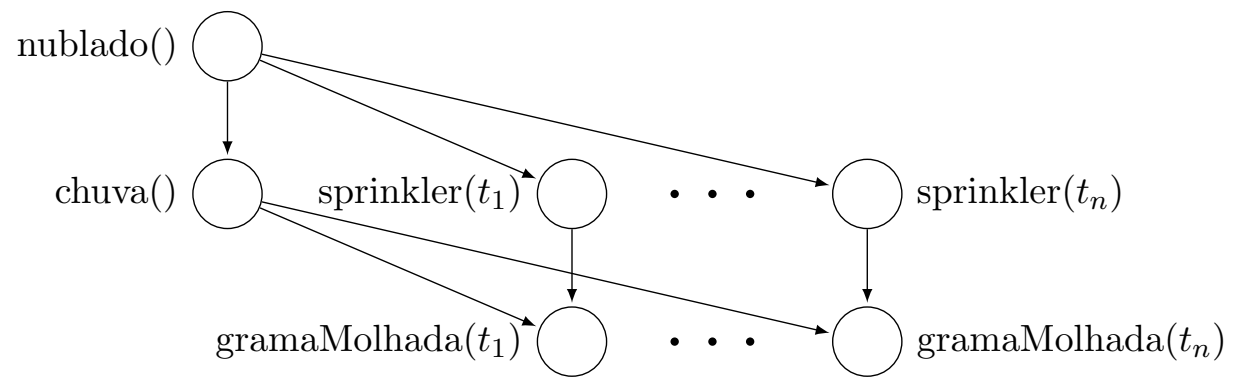

Figura 3: Versão proposicionalizada da rede Sprinkler

Na Figura 2 utilizou-se a notação de "chapas" (BUNTINE, 1994). O subgrafo envolvendo as variáveis sprinkler $(T)$ e gramaMolhada(T) estão circundadas por uma caixa 
(denominada "chapa") que indica que a estrutura do subgrafo se repete para cada indivíduo da população atrelada à variável lógica associada à chapa (no caso, T).

Exemplo 11. Considere a rede Sprinkler representada na Figura 2. A distribuição de probabilidade por ela representada pode ser traduzida pelo seguinte conjunto de parfactors ${ }^{2}$ :

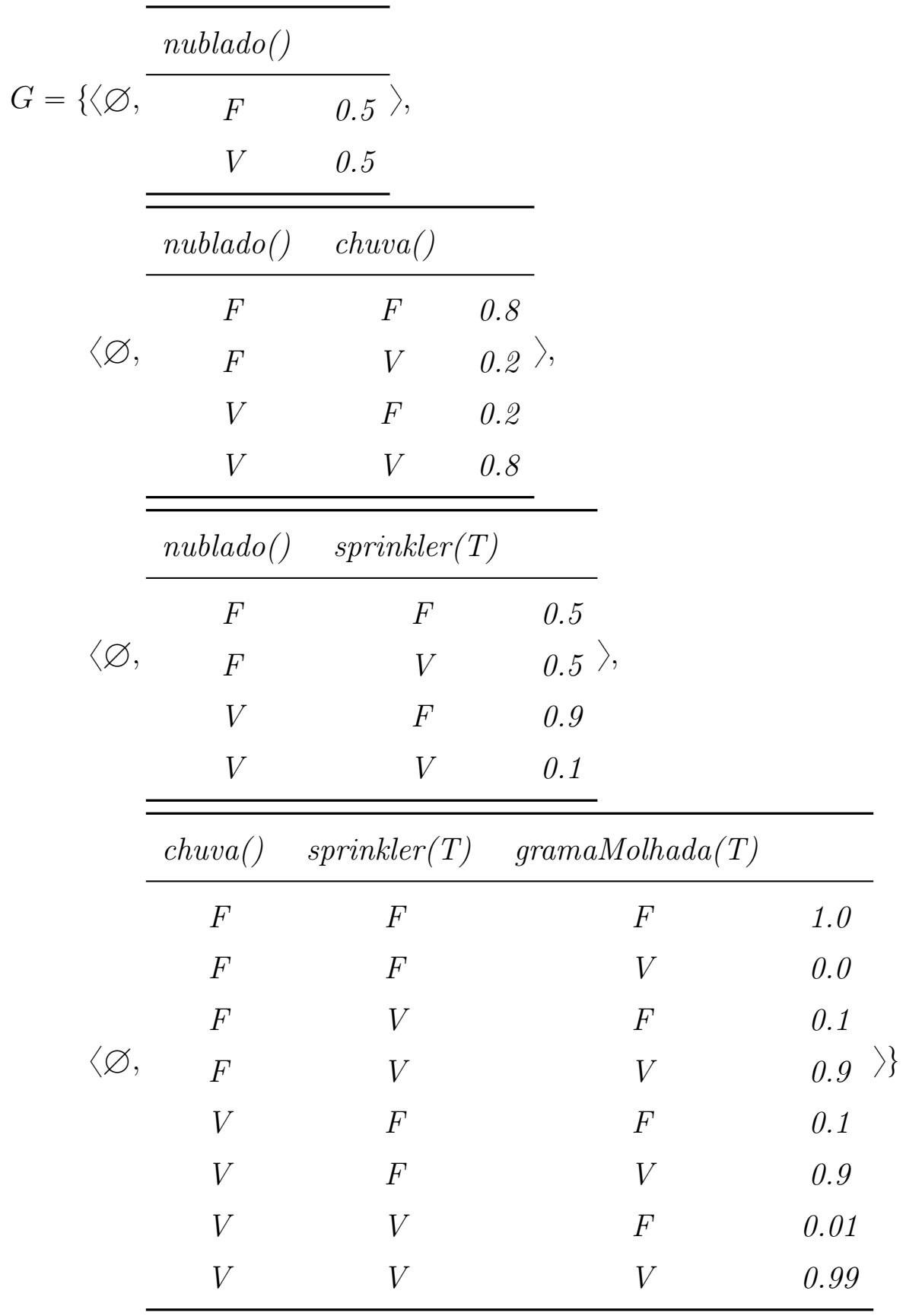

As restrições de desigualdade servem para armazenar conhecimento a respeito de certos indivíduos da população.

\footnotetext{
${ }^{2} \mathrm{~A}$ notação utilizada neste exemplo para representar parfactors consiste em dois elementos: o conjunto de restrições $\mathcal{C}$ e a representação tabular do fator listando as variáveis aleatórias parametrizadas de $\mathcal{V}$ e os valores de $\mathcal{F}$.
} 
Exemplo 12. Considere que no Exemplo 11 se deseja adicionar a informação de que o sprinkler do terreno $t_{1}$ está ligado. O parfactor 3.1 .5 deve ser substituído por dois parfactors:

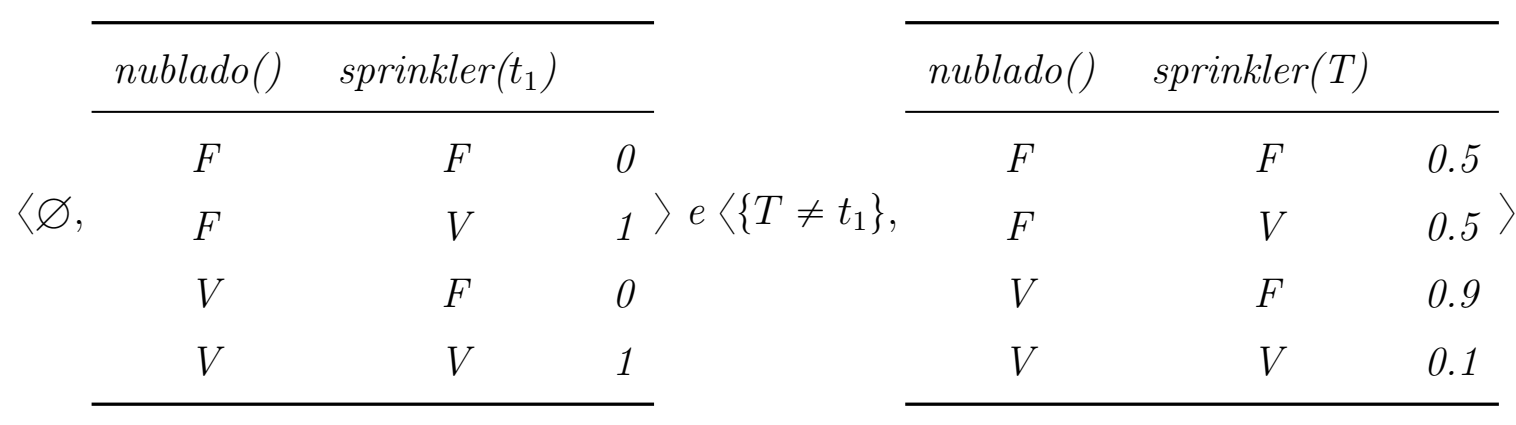

O Exemplo 13 foi extraído de (KISYNSKI, 2010) e apresenta o contraste entre o tamanho de um parfactor e o seu poder de expressividade.

Exemplo 13. Considere uma variável aleatória parametrizada gramaMolhada $(T)$ e a fórmula de contagem $\#_{T}[$ gramaMolhada]. A variável lógica $T$ está atrelada à população $\left\{t_{1}, \ldots, t_{n}\right\}$, e gramaMolhada é uma functor com domínio $\{\mathrm{F}, \mathrm{V}\}$.

$O$ parfactor $g_{1}=\left\langle\varnothing,\{\right.$ gramaMolhada $\left.(T)\}, \mathcal{F}_{1}\right\rangle$ representa $n$ funções discretas com valores iguais em cada variável aleatória do conjunto $\mathrm{RV}($ gramaMolhada $(T))$. O fator $\mathcal{F}_{1}$ tem tamanho 2 e representa uma função do conjunto $\{\mathrm{F}, \mathrm{V}\}$ para os reais.

O parfactor $g_{2}=\left\langle\varnothing,\left\{\#_{T}[\right.\right.$ gramaMolhada $\left.\left.]\right\}, \mathcal{F}_{2}\right\rangle$ representa uma função discreta cujos valores dependem apenas da quantidade de variáveis do conjunto RV(gramaMolhada $(T))$ que assumem um determinado valor do dominio $\{\mathrm{F}, \mathrm{V}\}$. O fator $\mathcal{F}_{2}$ tem tamanho $n+1$.

$O$ parfactor $g_{3}=\left\langle\varnothing,\left\{\right.\right.$ gramaMolhada $\left(t_{1}\right), \ldots$, gramaMolhada $\left.\left.\left(t_{n}\right)\right\}, \mathcal{F}_{3}\right\rangle$ pode representar qualquer função discreta nas variáveis $\mathrm{RV}($ gramaMolhada $(T))$. O fator $\mathcal{F}_{3}$ tem tamanho $2^{n}$.

Note que uma maior expressividade implica em fatores maiores. A Figura 4 ilustra a relação entre os conjuntos de fatores que cada parfactor representa. O tamanho dos círculos indica o tamanho dos fatores que cada parfactor possui. Para deixar claro que o parfactor $g_{1}$ representa $n$ funções idênticas, foram representados $n$ conjuntos, um para cada substituição possível na variável lógica $T$.

Um parfactor pode ser elevado a um número real. Considere um parfactor $g=\langle\mathcal{C}, \mathcal{V}, \mathcal{F}\rangle$. O fator $\mathcal{F}$ elevado a um número real $r$ é denotado por $\mathcal{F}^{r}$ (KISYNSKI, 2010), e para todas as atribuições de valores $\mathbf{v}$ às variáveis do conjunto $\mathcal{V}, \mathcal{F}^{r}(\mathbf{v})=(\mathcal{F}(\mathbf{v}))^{r}$. O parfactor $g$ elevado a um número real $r$ é interpretado como $g^{r}=\left\langle\mathcal{C}, \mathcal{V}, \mathcal{F}^{r}\right\rangle$ (KISYNSKI, 2010). 


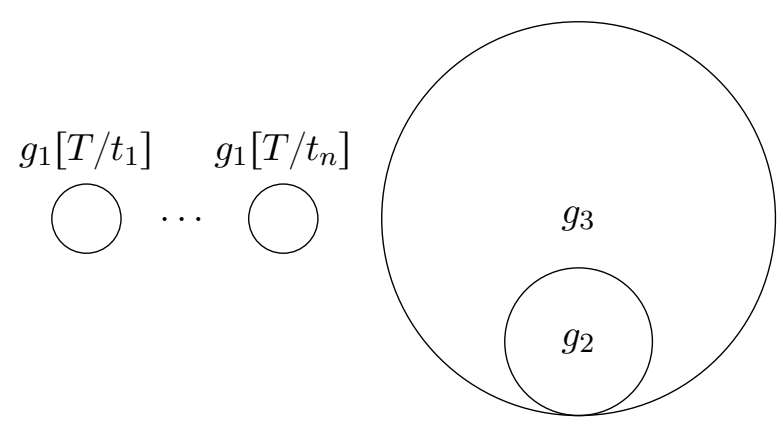

Figura 4: Relação entre os conjuntos de fatores dos parfactors do Exemplo 13

\subsubsection{Atribuição de valores}

O conceito de atribuição de valores será usado para definir fatores em variáveis aleatórias parametrizadas.

O símbolo v será usado para denotar uma atribuição de valores (MILCH et al., 2008). Assim, $\mathbf{v}(X)$ denota um valor da variável aleatória $X$. De maneira análoga, dada uma variável aleatória parametrizada $\mathrm{f}\left(t_{1}, \ldots, t_{k}\right), \mathbf{v}(f)$ denota uma atribuição de valores a todas as variáveis aleatórias do conjunto RV $(f)$ (KISYNSKI, 2010). Note que deve-se ter necessariamente $\mathbf{v}(f) \in \mathrm{RANGE}(f)$.

Exemplo 14. Seja $X$ uma variável aleatória booleana. Então $\mathbf{v}(X)=\mathrm{F}$ é uma possível atribuição de valor à variável $X$. Seja $f(A)$ uma variável aleatória parametrizada booleana, $\operatorname{com} \mathcal{D}(A)=\left\{a_{1}, a_{2}, a_{3}\right\}$. Então $\mathbf{v}(f)=\mathrm{F}$ é uma possível atribuição de valor à variável $f$ e é equivalente a $\mathbf{v}\left(f\left(a_{1}\right)\right)=\mathbf{v}\left(f\left(a_{2}\right)\right)=\mathbf{v}\left(f\left(a_{3}\right)\right)=\mathrm{F}$.

Atribuições de valores também serão utilizadas no contexto de fatores. Neste caso, v denota uma atribuição de valores a um conjunto de variáveis aleatórias (KISYNSKI, 2010). Seja $\mathcal{F}$ um fator nas variáveis aleatórias $\left\{X_{1}, \ldots, X_{n}\right\}$. Então $\mathcal{F}(\mathbf{v})$ é interpretado como $\mathcal{F}\left(\mathbf{v}\left(X_{1}\right), \ldots, \mathbf{v}\left(X_{n}\right)\right)$, isto é, $\mathcal{F}(\mathbf{v})$ é o valor de uma linha da representação tabular do fator $\mathcal{F}$. Uma interpretação semelhante também será dada a fatores em variáveis aleatórias parametrizadas. Seja $\mathcal{F}$ um fator nas variáveis aleatórias parametrizadas $\left\{f_{1}\right.$, $\left.\ldots, f_{n}\right\}$; então $\mathcal{F}(\mathbf{v})=\mathcal{F}\left(\mathbf{v}\left(f_{1}\right), \ldots, \mathbf{v}\left(f_{n}\right)\right)$. Se uma atribuição $\mathbf{v}$ atribui um valor a uma variável que não existe no fator $\mathcal{F}$, então, a atribuição é simplesmente ignorada.

Exemplo 15. Considere o fator $\mathcal{F}$ nas variáveis aleatórias parametrizadas $\{$ nublado( $)$, 
chuva()\} representado a seguir.

$\mathcal{F}=$\begin{tabular}{ccc}
\hline nublado() & chuva() & \\
\hline$F$ & $F$ & 0.8 \\
$F$ & $V$ & 0.3 \\
$V$ & $F$ & 0.2 \\
$V$ & $V$ & 0.7 \\
\hline
\end{tabular}

Seja $\mathbf{v}$ uma atribuição de valores às variáveis $\{\operatorname{sol}()$, chuva (), nublado( $)\}$ tal que $\mathbf{v}(\operatorname{sol}())=$ $\mathrm{F}, \mathbf{v}(\operatorname{chuva}())=\mathrm{F}, \mathbf{v}($ nublado ()$)=\mathrm{V}$. Então $\mathcal{F}(\mathbf{v})=0.2$ (note que a variável sol () não existe em $\mathcal{F}$, então ela foi ignorada).

\subsubsection{Parfactors de agregação}

Em modelos direcionados de primeira ordem, pode ocorrer a necessidade de representar o efeito agregado que um nó pai exerce sobre um nó filho. Suponha que se deseja medir a probabilidade de existir um ganhador num sorteio de loteria. Se ninguém apostar, evidentemente esta probabilidade é nula. Uma possível rede Bayesiana que modela esta situação é ilustrada na Figura 5.

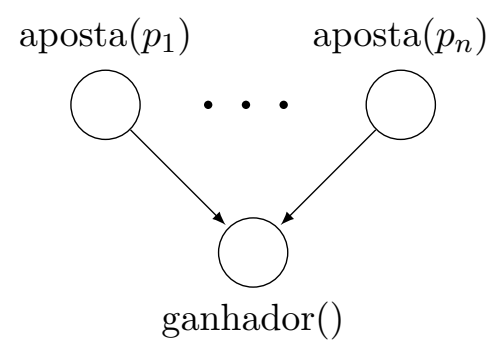

Figura 5: Rede Bayesiana que representa uma relação entre apostadores e ganhadores

Nesta rede, existem $n$ nós aposta $\left(p_{i}\right)$, que representam a probabilidade da pessoa $p_{i}$ ter apostado na loteria, e um nó ganhador(), que representa a probabilidade de existir um ganhador no sorteio.

Note que a probabilidade de existir um ganhador aumenta conforme o número de pessoas que apostam aumenta, isto é, existe um efeito agregado dos nós aposta $\left(p_{i}\right)$ sobre o nó ganhador(). A rede da Figura 5 pode ser transformada em um modelo de primeira ordem atrelando o conjunto de pessoas $\left\{p_{1}, \ldots, p_{n}\right\}$ a uma variável lógica $P$. A agregação é representada por uma flecha "cortada". A Figura 6 ilustra o modelo de primeira ordem equivalente à rede da Figura 5. 


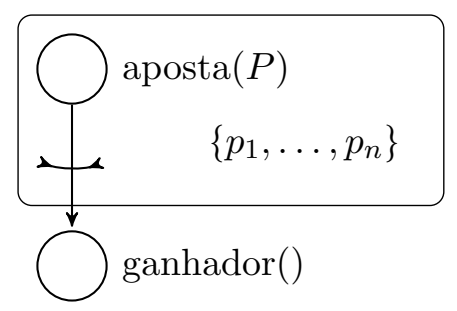

Figura 6: Modelo de primeira ordem equivalente à rede da Figura 5

O efeito de agregação ocorre em modelos de primeira ordem sempre que um nó possui variáveis lógicas extras em relação ao nó filho. Quando isto acontece, a proposicionalização do modelo leva ao surgimento de uma quantidade de nós pais que depende do tamanho da população atrelada às variáveis lógicas extras. No exemplo da Figura 5, o número de pais do nó ganhador() depende do tamanho da população de apostadores. Isto representa um problema para inferências em primeira ordem, pois os parfactors definidos na Seção 3.1.3 não são capazes de representar esta relação de maneira independente do tamanho da população. De fato, as duas únicas possibilidades são os parfactors $\langle\mathcal{C}$, $\left\{\#_{P}[\operatorname{aposta}()]\right.$, ganhador ()$\left.\}, \mathcal{F}_{1}\right\rangle$ e $\left\langle\mathcal{C},\left\{\operatorname{aposta}\left(p_{1}\right), \ldots, \operatorname{aposta}\left(p_{n}\right), \operatorname{ganhador}()\right\}, \mathcal{F}_{2}\right\rangle$, e ambos possuem fatores que dependem do número de pessoas $n$.

Diante deste problema, Kisynski e Poole (2009) propuseram a criação de uma estrutura de dados denominada parfactors de agregação, que são extensões de parfactors capazes de representar agregações de maneira independente do tamanho das populações atreladas às variáveis lógicas extras do nó pai.

No restante deste trabalho, supõe-se que os modelos probabilísticos de primeira ordem satisfazem as seguintes condições (KISYNSKI, 2010):

1. Para cada variável aleatória parametrizada, seu nó pai tem no máximo uma variável lógica extra;

2. Se uma variável aleatória parametrizada $\mathrm{c}(\ldots)$ tem um pai $\mathrm{p}(\ldots, A, \ldots)$ com uma variável lógica extra $A$, então:

(a) $\mathrm{p}(\ldots, A, \ldots)$ é único pai de $\mathrm{c}(\ldots)$;

(b) O domínio de $p$ é um subconjunto do domínio de $c$;

(c) $\mathrm{c}(\ldots)$ é uma função determinística de seu pai: $\mathrm{c}(\ldots)=\mathrm{p}\left(\ldots, a_{1}, \ldots\right) \otimes \cdots \otimes$ $\mathrm{p}\left(\ldots, a_{n}, \ldots\right)=\otimes_{a \in \mathcal{D}(A)} \mathrm{p}(\ldots, a, \ldots)$, em que $\otimes$ é um operador binário comutativo e associativo sobre o domínio de $\mathrm{c}(\ldots)$.

Não há necessidade de definir agregações sobre mais de uma variável lógica, pois o 
operador $\otimes$ é comutativo e associativo. Distribuições mais complicadas podem ser obtidas pela introdução de variáveis aleatórias parametrizadas auxiliares e pela combinação de agregações. Este fato é ilustrado no Exemplo 16, extraído de Kisynski (2010).

Exemplo 16. Considere uma variável aleatória parametrizada $p(A, B, C)$ que tem como filha a variável aleatória parametrizada $c(C)$. Seja $\otimes$ um operador de agregação em $A$ e $B$ :

$$
c(C)=\bigotimes_{(a, b) \in \mathcal{D}(A) \times \mathcal{D}(B)} p(A, B, C) .
$$

Defina uma variável aleatória parametrizada $c^{\prime}(B, C)$ que possui o mesmo domínio de $c$, tal que

$$
c^{\prime}(B, C)=\bigotimes_{a \in \mathcal{D}(A)} p(A, B, C)
$$

Então

$$
c(C)=\bigotimes_{b \in \mathcal{D}(B)} c^{\prime}(B, C)
$$

Tendo em vista as condições sob as quais agregações estão submetidas, a Definição 6 formaliza o conceito de parfactor de agregação.

Definição 6. Um parfactor de agregação (KISYNSKI; POOLE, 2009) é uma séptupla $\langle\mathcal{C}, p$, $\left.c, V, \mathcal{F}_{p}, \otimes, A\right\rangle$, em que:

- $p$ e c são variáveis aleatórias parametrizadas tais que

$-\operatorname{RANGE}(p) \subseteq \operatorname{RANGE}(c) ;$

- PARAM $(p) \backslash \operatorname{PARAM}(c)=\{A\} ;$

- A é a única variável lógica de p ausente em c;

- $\mathcal{C}$ é um conjunto de restrições tal que $\mathcal{D}(A): \mathcal{C} \neq \varnothing$;

- $\otimes$ é um operador binário comutativo e associativo em R A NGE(c);

- $V=\left\{f_{1}, \ldots, f_{n}\right\}$ é um conjunto de variáveis aleatórias parametrizadas tal que

- Para todo par de variáveis $\left(f_{i}, f_{j}\right)$ de $V,\left(\operatorname{RV}\left(f_{i}\right): \mathcal{C}\right) \cap\left(\operatorname{RV}\left(f_{j}\right): \mathcal{C}\right)=\varnothing$;

- Para toda variável $f_{i}$ de $V,\left(\operatorname{RV}\left(f_{i}\right): \mathcal{C}\right) \cap(\operatorname{RV}(p): \mathcal{C})=\varnothing ;$

- Para toda variável $f_{i}$ de $V,\left(\operatorname{RV}\left(f_{i}\right): \mathcal{C}\right) \cap(\operatorname{RV}(c): \mathcal{C})=\varnothing ;$

- $\mathcal{F}_{p}$ é um fator que representa uma função $f: p \times f_{1} \times \cdots \times f_{n} \rightarrow \mathbb{R}$. 
Um parfactor de agregação $g_{A}=\left\langle\mathcal{C}, p, c, V, \mathcal{F}_{p}, \otimes, A\right\rangle$ representa um conjunto de fatores, um fator $\mathcal{F}_{p v c}$ para cada substituição elementar nas variáveis lógicas que aparecem em $g$ (com exceção da variável lógica $A$ ) satisfazendo as restrições em $\mathcal{C}$. Seja $\mathcal{D}(A): \mathcal{C}=\left\{a_{1}\right.$, $\left.\ldots, a_{n}\right\}$ e $V=\left\{f_{1}, \ldots, f_{m}\right\}$. Cada fator $\mathcal{F}_{p v c}$ é uma função $f: p\left[A / a_{1}\right] \times \cdots \times p\left[A / a_{n}\right] \times$ $f_{1} \times \cdots \times f_{m} \times c \rightarrow \mathbb{R}$. Seja $r_{c}=|\operatorname{RV}(c): \mathcal{C}|$ e $r_{p}=|\operatorname{RV}(p[A / a]): \mathcal{C}|$, para algum $a \in \mathcal{D}(A): \mathcal{C}$. Para toda atribuição de valores $\mathbf{v}$ às variáveis aleatórias parametrizadas do conjunto $\left\{p\left[A / a_{1}\right], \ldots, p\left[A / a_{n}\right], f_{1}, \ldots, f_{m}, c\right\}, \mathcal{F}_{p v c}(\mathbf{v})$ é definido por

$$
\mathcal{F}_{p v c}(\mathbf{v})= \begin{cases}\prod_{a \in \mathcal{D}(A): \mathcal{C}} \mathcal{F}_{p}^{\frac{r_{p}}{r_{c}}}\left(p[A / a], f_{1}, \ldots, f_{m}\right), & \text { se } \bigotimes_{a \in \mathcal{D}(A): \mathcal{C}} \mathbf{v}(p[A / a])=\mathbf{v}(c) \\ 0, & \text { caso contrário. }\end{cases}
$$

O expoente $\frac{r_{p}}{r_{c}}$ é um fator corretivo para os casos em que a variável c(...) é parametrizada por variáveis lógicas ausentes em $\mathrm{p}(\ldots, A, \ldots)$. Note que, quando a variável $c$ não possui variáveis lógicas extras em relação à variável $p$, tem-se $\frac{r_{p}}{r_{c}}=1$.

Na definição de modelos de primeira ordem, os parfactors de agregação sempre terão um fator $\mathcal{F}_{p}$ constante com valor 1 para todas as possíveis atribuições de valores $\mathbf{v}$ às variáveis aleatórias parametrizadas de $\mathcal{F}_{p}$. Este fator será denotado por $\mathbb{1}$.

Exemplo 17. Considere a rede da Figura 6. Um possível parfactor de agregação para o nó ganhador () é $g_{A}=\langle\varnothing, \operatorname{aposta}(P)$,ganhador ()$, \varnothing, \mathbb{1}, \mathrm{oR}, P\rangle$, em que

$$
\mathbb{1}=\begin{array}{cc}
\hline \operatorname{aposta}(P) \\
\hline F & 1 \\
V & 1 \\
\hline
\end{array}
$$

Suponha que $\mathcal{D}(P)=\{$ Ana,Bruno $\}$. Então $g_{A}$ representa o fator

\begin{tabular}{cccc}
\hline aposta(Ana) & aposta(Bruno) & ganhador() & \\
\hline$F$ & $F$ & $F$ & 1 \\
$F$ & $F$ & $V$ & 0 \\
$F$ & $V$ & $F$ & 0 \\
$F$ & $V$ & $V$ & 1 \\
$V$ & $F$ & $F$ & 0 \\
$V$ & $F$ & $V$ & 1 \\
$V$ & $V$ & $F$ & 0 \\
$V$ & $V$ & $V$ & 1 \\
\hline
\end{tabular}


No restante deste trabalho, o termo "parfactor na forma padrão" se referirá aos parfactors definidos na Seção 3.1.3; e o termo "parfactor" será utilizado para se referir tanto aos parfactors na forma padrão como aos parfactors de agregação.

\subsubsection{Restrições na forma normal}

Em geral, dada uma variável lógica $X$ e um conjunto de restrições de desigualdade $\mathcal{C}$, o tamanho do conjunto $\mathcal{D}(X): \mathcal{C}$ dependerá de outras variáveis lógicas presentes em $\mathcal{C}$.

Exemplo 18. Considere duas variáveis lógicas $X$ e $Y$ tais que $\mathcal{D}(X)=\mathcal{D}(Y)=\left\{x_{1}, \ldots\right.$, $\left.x_{n}\right\}$. Seja $\mathcal{C}$ o conjunto de restrições de desigualdade $\left\{X \neq x_{1}, X \neq Y\right\}$. Então:

- $|\mathcal{D}(X): \mathcal{C}|=n-2$ quando $Y \neq x_{1}$

- $|\mathcal{D}(X): \mathcal{C}|=n-1$ quando $Y=x_{1}$.

Este tipo de variação no tamanho do conjunto de indivíduos que satisfazem determinadas restrições não é desejável para realização de inferências, pois o domínio de uma fórmula de contagem depende do tamanho deste conjunto. Para evitar esta dificuldade, Milch et al. (2008) impuseram que conjuntos de restrições estejam na forma normal.

Definição 7. Seja $\mathcal{C}$ um conjunto de restrições de desigualdade e $X$ uma variável lógica. $O$ conjunto excluído de $X$ em $\mathcal{C}$ (MILCH et al., 2008), denotado por $E_{X}^{\mathcal{C}}$, é o conjunto dos termos $t$ tais que a restrição $X \neq t$ pertence a $\mathcal{C}$.

Exemplo 19. Seja $\mathcal{C}=\left\{X \neq Y, X \neq x_{1}, X \neq x_{2}\right\}$ um conjunto de restrições de desigualdade. O conjunto excluído de $X$ em $\mathcal{C}$ é dado por $E_{X}^{\mathcal{C}}=\left\{Y, x_{1}, x_{2}\right\}$.

Definição 8. Seja $\mathcal{C}$ um conjunto de restrições de desigualdade. O conjunto $\mathcal{C}$ está na forma normal (MILCH et al., 2008) se, para cada restrição de desigualdade envolvendo duas variáveis lógicas $X \neq Y$ em $\mathcal{C}$, tem-se $E_{X}^{\mathcal{C}} \backslash\{Y\}=E_{Y}^{\mathcal{C}} \backslash\{X\}$.

Exemplo 20. Seja $\mathcal{C}=\left\{X \neq Y, X \neq x_{1}, X \neq x_{2}\right\}$ um conjunto de restrições de desigualdade. Tem-se $E_{X}^{\mathcal{C}}=\left\{Y, x_{1}, x_{2}\right\}$ e $E_{Y}^{\mathcal{C}}=\{X\}$. Como $E_{X}^{\mathcal{C}} \backslash\{Y\} \neq E_{Y}^{\mathcal{C}} \backslash\{X\}$, o conjunto $\mathcal{C}$ não está na forma normal.

Seja agora $\mathcal{C}=\left\{X \neq Y, X \neq x_{1}, Y \neq x_{1}\right\}$. Tem-se $E_{X}^{\mathcal{C}}=\left\{Y, x_{1}\right\}$ e $E_{Y}^{\mathcal{C}}=\left\{X, x_{1}\right\}$, e portanto o conjunto $\mathcal{C}$ está na forma normal.

Definição 9. Seja $g=\langle\mathcal{C}, \mathcal{V}, \mathcal{F}\rangle$ um parfactor e $\mathcal{C}^{\prime}$ o conjunto formado pela união do conjunto $\mathcal{C}$ e dos conjuntos de restrições de fórmula de contagem em $\mathcal{V}$. Então o parfactor $g$ está na formal normal (KISYNSKI, 2010) se $\mathcal{C}^{\prime}$ está na forma normal. 
A Proposição 1 formaliza o cálculo do tamanho da população atrelada a uma variável lógica sujeita a restrições na forma normal. A demonstração que a segue foi proposta pelo autor deste trabalho.

Proposição 1. Seja $\mathcal{C}$ um conjunto de restrições na forma normal e $X$ uma variável lógica. Então

$$
|\mathcal{D}(X): \mathcal{C}|=|\mathcal{D}(X)|-\left|E_{X}^{\mathcal{C}}\right|
$$

Demonstração. Seja:

- $\mathcal{C}_{X}$ o subconjunto de $\mathcal{C}$ que contém as restrições na variável lógica $X$;

- $E_{C}$ o conjunto de constantes nas restrições de $\mathcal{C}_{X}$;

- $E_{L}$ o conjunto de constantes nas restrições de $\mathcal{C}_{X}$;

- $\theta$ uma substituição elementar nas variáveis lógicas de $E_{L}$;

- $E_{L} \theta$ o resultado da aplicação da substituição $\theta$ a $E_{L}$

Como o conjunto $\mathcal{C}$ está na forma normal, $E_{L} \theta \cap E_{C}=\varnothing$ e $\left|E_{L}\right|=\left|E_{L} \theta\right|$. Pela definição de conjunto excluído, $E_{X}^{\mathcal{C}}=E_{L} \cup E_{C}$. Então

$$
\left|E_{X}^{\mathcal{C}}\right|=\left|E_{L} \cup E_{C}\right|=\left|E_{L}\right|+\left|E_{C}\right|=\left|E_{L} \theta\right|+\left|E_{C}\right|=\left|E_{L} \theta \cup E_{C}\right|
$$

O conjunto $\mathcal{D}(X): \mathcal{C}$ pode ser reescrito como:

$$
\mathcal{D}(X): \mathcal{C}=\mathcal{D}(X): \mathcal{C}_{X}=\mathcal{D}(X) \backslash\left(E_{C} \cup E_{L} \theta\right)
$$

E portanto:

$$
\begin{aligned}
|\mathcal{D}(X): \mathcal{C}| & =\left|\mathcal{D}(X) \backslash\left(E_{C} \cup E_{L} \theta\right)\right| \\
& =|\mathcal{D}(X)|-\left|E_{C} \cup E_{L} \theta\right|, \text { pois } E_{C} \cup E_{L} \theta \subseteq \mathcal{D}(X) \\
& =|\mathcal{D}(X)|-\left|E_{X}^{\mathcal{C}}\right|
\end{aligned}
$$

\subsection{Operações elementares}

O A C-F OVE utiliza o algoritmo C-F OVE (MILCH et al., 2008) como base, incorporando o conceito de parfactors de agregação quando necessário e incluindo o tratamento destes 
nas operações elementares.

Seja $G$ um conjunto de parfactors. O produto de todos os fatores representados por parfactors do conjunto $G$ será denotado por $\mathcal{J}(G)$. O conjunto de todas as variáveis aleatórias representadas por variáveis aleatórias parametrizadas em parfactors de $G$ será simbolizado por U. O conjunto de variáveis aleatórias de consulta será representado por $\mathbf{Q}$; note que $\mathbf{Q} \subseteq \mathbf{U}$.

O marginal de $\mathcal{J}(G)$ em $\mathbf{Q}$, denotado por $\mathcal{J}_{\mathbf{Q}}(G)$, é o resultado da eliminação de todas as variáveis do conjunto $\mathbf{U} \backslash \mathbf{Q}$, isto é,

$$
\mathcal{J}_{\mathbf{Q}}(G)=\sum_{\mathbf{U} \backslash \mathbf{Q}} \mathcal{J}(G)
$$

O algoritmo A C-F OVE calcula o marginal $\mathcal{J}_{\mathbf{Q}}(G)$ utilizando eliminação em primeira ordem sempre que possível. Como foi visto no Exemplo 12, as variáveis aleatórias observadas podem ser adicionadas pela inclusão de parfactors no conjunto $G$. A eliminação em primeira ordem é possível somente se algumas condições forem satisfeitas. Por este motivo, existem outras operações elementares que visam transformar o conjunto de parfactors de maneira que este satisfaça estas condições.

A eliminação em primeira ordem será a primeira operação a ser abordada. Em seguida, serão descritas as operações habilitadoras. As operações descritas nesta seção serão denominadas operações elementares, pois estas constituirão as peças fundamentais para o algoritmo AC-FOVE.

Um detalhe importante sobre as operações elementares aplicadas em parfactors de agregação: Kisynski (2010) dividiu o tratamento de parfactors de agregação em duas partes: uma versão "simplificada" dos parfactors de agregação, que não possuem variáveis aleatórias parametrizadas de contexto, e uma versão "generalizada" dos parfactors de agregação, que possuem um conjunto de variáveis de contexto. As operações elementares sobre os parfactors de agregação (eliminação, multiplicação e divisão) foram definidas por Kisynski (2010) apenas para a versão simplificada dos parfactors de agregação. Neste trabalho estas operações serão definidas para a versão generalizada dos parfactors de agregação, que também englobam a versão simplificada. 


\subsubsection{Eliminação}

Eliminar uma variável aleatória parametrizada de um parfactor na forma padrão é semelhante a eliminar uma variável aleatória de um fator (também conhecido como sumout de uma variável). Como uma variável aleatória parametrizada representa um conjunto de variáveis aleatórias, será necessário considerar o tamanho deste conjunto para criar correções que mantenham os resultados consistentes. Além disso, existem certas condições que devem ser satisfeitas para que a eliminação em primeira ordem possa ser feita. A eliminação em parfactors na forma padrão e a eliminação em parfactors de agregação serão tratadas separadamente nesta seção.

Um aspecto a se considerar na eliminação em primeira ordem é a forma da variável aleatória parametrizada que se deseja eliminar. Quando a variável for uma fórmula de contagem, é necessário considerar que cada histograma de seu domínio também representa um conjunto de valores de variáveis aleatórias.

Exemplo 21. Considere a fórmula de contagem $\gamma=\#_{X}[f(X)]$, com $\mathcal{D}(X)=\left\{x_{1}, x_{2}, x_{3}\right\}$ $e \operatorname{RANGE}(f)=\{\mathrm{F}, \mathrm{V}\}$. Considere ainda o histograma $\left(\#_{\mathrm{F}}=2, \#_{\mathrm{V}}=1\right)$ de $\operatorname{RANGE}(\gamma)$. Este histograma representa três diferentes atribuições de valores:

\begin{tabular}{|c|c|c|c|c|c|c|}
\hline & & & \\
\hline & & & $f\left(x_{1}\right)$ & $f\left(x_{2}\right)$ & $f\left(x_{3}\right)$ & \\
\hline \multicolumn{2}{|l|}{$\#_{X}[f(X)]$} & & & $\vdots$ & & $\vdots$ \\
\hline$\vdots$ & $\vdots$ & $=$ & $F$ & $F$ & $V$ & 0.3 \\
\hline$\left(\#_{\mathrm{F}}=2, \#_{\mathrm{V}}=1\right)$ & 0.3 & & $F$ & $V$ & $F$ & 0.3 \\
\hline$\vdots$ & $\vdots$ & & $V$ & $F$ & $F$ & 0.3 \\
\hline
\end{tabular}

O número de atribuições de valores que um histograma representa pode ser obtido por análise combinatória. Considere o histograma $h=\left(k_{1}, \ldots, k_{m}\right)$. Como mencionado na Seção 3.1.2, a soma $k_{1}+\cdots+k_{m}$ sempre será constante para uma dada fórmula de contagem. O número de atribuições de valores será então igual ao número de maneiras de distribuir $k_{1}+\cdots+k_{m}$ valores em $m$ elementos do histograma, dado pelo coeficiente multinomial:

$$
\left(\begin{array}{c}
k_{1}+\cdots+k_{m} \\
k_{1}, \ldots, k_{m}
\end{array}\right)=\frac{\left(k_{1}+\cdots+k_{m}\right) !}{k_{1} ! \cdots k_{m} !}
$$

Utilizando a fórmula do coeficiente multinomial, pode-se derivar o número de atribuições que um histograma estabelece. 
Definição 10. Considere uma fórmula de contagem $\gamma=\#_{X: \mathcal{C}_{X}}[f(X)]$ e um histograma $h$ em R A N GE $(\gamma)$. Denotar-se-á por N u M-ATR IB $(\gamma, h)$ o número de atribuições de valores que o histograma $h$ representa para a fórmula de contagem $\gamma$ (MILCH et al., 2008):

$$
\operatorname{Num}-\operatorname{Atrib}(\gamma, h)=\frac{\left|\mathcal{D}(X): \mathcal{C}_{X}\right| !}{\prod_{u \in \operatorname{RANGE}(f)}(h(u) !)}
$$

Exemplo 22. Considere a fórmula de contagem $\gamma=\#_{X}[f(X)] \operatorname{com} \mathcal{D}(X)=\left\{x_{1}, x_{2}, x_{3}\right\}$ $e \operatorname{RANGE}(f)=\{\mathrm{F}, \mathrm{V}\}$. Considere ainda o histograma $\left(\#_{\mathrm{F}}=2, \#_{\mathrm{V}}=1\right)$ de $\operatorname{RANGE}(\gamma)$. O número de atribuições que este histograma representa é:

$$
\mathrm{Num}-\operatorname{AtriB}\left(\#_{X}[f(X)], h\right)=\frac{3 !}{2 ! \cdot 1 !}=3,
$$

o que corresponde às atribuições $(\mathrm{F}, \mathrm{F}, \mathrm{V}),(\mathrm{F}, \mathrm{V}, \mathrm{F}),(\mathrm{V}, \mathrm{F}, \mathrm{F})$.

Definição 11. Considere uma variável aleatória parametrizada $f$ que não seja uma fórmula de contagem e um elemento $h$ em RANGE $(f)$. O valor de NuM-ATRIB $(f, h)$ será sempre 1.

Um outro aspecto a se considerar na eliminação em primeira ordem são correções que dependem do tamanho do conjunto de variáveis aleatórias representadas pelas variáveis aleatórias parametrizadas dos parfactors. Isto é necessário porque a eliminação de uma variável lógica $X$ de um parfactor envolve uma proposicionalização implícita desta variável.

Exemplo 23. Considere o parfactor $g=\langle\varnothing,\{f(), h(X)\}, \mathcal{F}\rangle$ e suponha que $\mathcal{D}(X)=$ $\left\{x_{1}, x_{2}\right\}$. O parfactor $g$ representa dois fatores: $\mathcal{F}\left(f(), h\left(x_{1}\right)\right)$ e $\mathcal{F}\left(f(), h\left(x_{2}\right)\right)$. Eliminar a variável aleatória parametrizada $h(X)$ é equivalente a fazer duas eliminações em nível proposicional:

$$
\begin{aligned}
\sum_{h(X)} \mathcal{J}(g) & =\sum_{h\left(x_{1}\right), h\left(x_{2}\right)} g\left[X / x_{1}\right] \odot g\left[X / x_{2}\right] \\
& =\sum_{h\left(x_{1}\right)} g\left[X / x_{1}\right] \odot \sum_{h\left(x_{2}\right)} g\left[X / x_{2}\right] \\
& =\mathcal{F}^{\prime} \odot \mathcal{F}^{\prime}
\end{aligned}
$$

Ao final, obteremos o fator $\mathcal{F}^{\prime}=\sum_{g(X)} \mathcal{F}$ elevado a 2, que é o tamanho do conjunto $\mathcal{D}(X): \varnothing$.

O expoente que mantém o resultado consistente para a eliminação em primeira ordem pode ser deduzido a partir do Exemplo 23. Suponha que se deseja eliminar uma variável aleatória parametrizada $f$ de um parfactor $g=\langle\mathcal{C}, \mathcal{V}, \mathcal{F}\rangle$. Seja $\left\{X_{1}, \ldots, X_{n}\right\}$ o conjunto de 
variáveis lógicas que aparecem apenas na variável aleatória parametrizada $f$. A eliminação de $f$ resulta em um parfactor na forma $\bar{g}=\langle\overline{\mathcal{C}}, \overline{\mathcal{V}}, \overline{\mathcal{F}}\rangle$, em que $\overline{\mathcal{C}}$ é o subconjunto de $\mathcal{C}$ com as restrições que não envolvem as variáveis lógicas extras de $f,\left\{X_{1}, \ldots, X_{n}\right\}, \overline{\mathcal{V}}$ é o conjunto $\mathcal{V}$ sem a variável $f$ e $\overline{\mathcal{F}}=\sum_{f} \mathcal{F}$. O parfactor $g$ representa $|g| /|\bar{g}|$ mais fatores que $\bar{g}$. Logo, para compensar a redução no número de fatores representados, o parfactor $\bar{g}$ deve ser elevado a $|g| /|| \bar{g} \mid$.

A Proposição 2 trata da eliminação de variáveis aleatórias parametrizadas em parfactors na forma padrão. Ela deriva do trabalho de Milch et al. (2008) e foi extraída e adaptada de Kisynski (2010).

Proposição 2. Seja $g=\langle\mathcal{C}, \mathcal{V}, \mathcal{F}\rangle$ um parfactor pertencente a um conjunto de parfactors G. Seja $f$ uma variável aleatória parametrizada de $\mathcal{V}$. Suponha que:

1. O parfactor g esteja na forma normal;

2. Nenhum outro parfactor de $G$ possua variáveis aleatórias representadas por $f$;

3. Todas as variáveis lógicas do parfactor g estejam inclusas na variável $f$.

Defina um parfactor $\bar{g}=\langle\overline{\mathcal{C}}, \overline{\mathcal{V}}, \overline{\mathcal{F}}\rangle$ tal que:

- $\overline{\mathcal{C}}=\mathcal{C}$

- $\overline{\mathcal{V}}=\mathcal{V} \backslash\{f\} ;$

- Para cada atribuição de valor $\mathbf{v}$ às variáveis de $\mathcal{V}$, tem-se

$$
\overline{\mathcal{F}}(\mathbf{v})=\sum_{h \in \operatorname{RANGE}(f)} \mathrm{NUM}-\operatorname{ATRIB}(f, h) \times \mathcal{F}(\mathbf{v})
$$

Seja ainda $r=|g| /|\bar{g}|$. Então:

$$
\sum_{\operatorname{Rv}(f): \mathcal{C}} \mathcal{J}(G)=\mathcal{J}\left(G \backslash\{g\} \cup\left\{\bar{g}^{r}\right\}\right)
$$

O Exemplo 24 foi extraído e adaptado de Kisynski (2010) e ilustra a eliminação em primeira ordem de uma variável aleatória parametrizada.

Exemplo 24. Considere as variáveis aleatórias parametrizadas $f(A, B), h(B), e(C)$, todas com dominio booleano. Suponha que todas as variáveis lógicas possuem uma população de 
tamanho n. Seja $G$ o seguinte conjunto de parfactors:

$$
\begin{aligned}
& G=\left\{g_{1}, g_{2}\right\}
\end{aligned}
$$

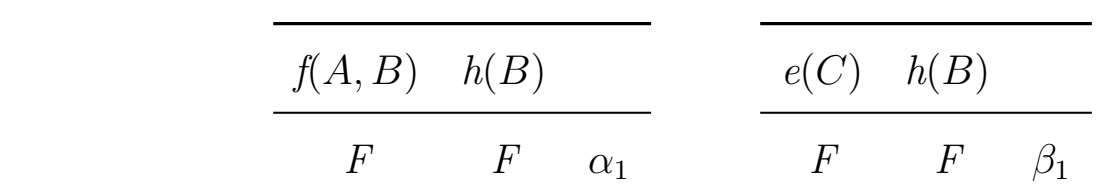

$$
\begin{aligned}
& =\left\{\left\langle\{A \neq B\}, \quad F \quad V \quad \alpha_{2}\right\rangle,\left\langle\varnothing, \quad F \quad \begin{array}{lll} 
& & \beta_{2}
\end{array}\right\}\right.
\end{aligned}
$$

$\begin{array}{cccccc}V & F & \alpha_{3} & V & F & \beta_{3} \\
V & V & \alpha_{4}\end{array} \quad$\begin{tabular}{lll}
$V$ & $V$ & $\beta_{4}$ \\
\hline
\end{tabular}

Assuma que se deseja calcular $\sum_{\mathrm{Rv}(f(A, B)):\{A \neq B\}} \mathcal{J}(G)$. Para isso é necessário eliminar a variável $f(A, B)$ de $g_{1}$. Note que todas as condições da Proposição 2 são satisfeitas:

- O parfactor $g_{1}$ está na forma normal pois $E_{A}^{\mathcal{C}} \backslash\{B\}=E_{B}^{\mathcal{C}} \backslash\{A\}$;

- $O$ parfactor $g_{2}$ não contém nenhuma variável aleatória representada por $\mathrm{RV}(f)$ : $\{A \neq B\}$

- Todas as variáveis lógicas de $g_{1}$ estão contidas em $f$.

Define-se um parfactor intermediário $\bar{g}$ :

$$
\begin{aligned}
& \bar{g}=\left\langle\{A \neq B\}, \overline{\frac{h(B)}{F} \alpha_{1}+\alpha_{3}}\right\rangle . \\
& \begin{array}{ll}
V \quad \alpha_{2}+\alpha_{4} \\
\hline
\end{array}
\end{aligned}
$$

Note que a variável lógica A está ausente do parfactor $\bar{g}$. Desse modo, é necessário aplicar o expoente corretivo: o parfactor g representa $n(n-1)$ fatores, enquanto que o parfactor $\bar{g}$ representa $n$ fatores; logo $g$ representa $n-1$ vezes mais fatores que $\bar{g}$. Assim, o resultado corrigido será

$$
\begin{aligned}
& \overline{\bar{g}}=\left\langle\{A \neq B\}, \overline{\left.\frac{h(B)}{F \quad\left(\alpha_{1}+\alpha_{3}\right)^{n-1}}\right\rangle} .\right. \\
& \text { V } \quad\left(\alpha_{2}+\alpha_{4}\right)^{n-1}
\end{aligned}
$$

E, pela Proposição 2,

$$
\sum_{\operatorname{Rv}(f(A, B)):\{A \neq B\}} \mathcal{J}(G)=\mathcal{J}\left(\overline{\bar{g}}, g_{2}\right)
$$

O Exemplo 25 ilustra a eliminação de uma fórmula de contagem. 
Exemplo 25. Considere um conjunto de parfactors $G$ com um único elemento, dado por $g=\left\langle\varnothing,\left\{\#_{A: \mathcal{C}_{A}}[f(A)], h(B)\right\}, \mathcal{F}\right\rangle$. Mostra-se o cálculo de $\sum_{\mathrm{Rv}\left(\#_{A: \mathcal{C}_{A}}[f(A)]\right)} \mathcal{J}(G)$ para quando o tamanho de $\mathcal{D}(A): \mathcal{C}_{A}$ é igual a 1, 2 e $n$.

$\operatorname{Seja}\left|\mathcal{D}(A): \mathcal{C}_{A}\right|=1$ :

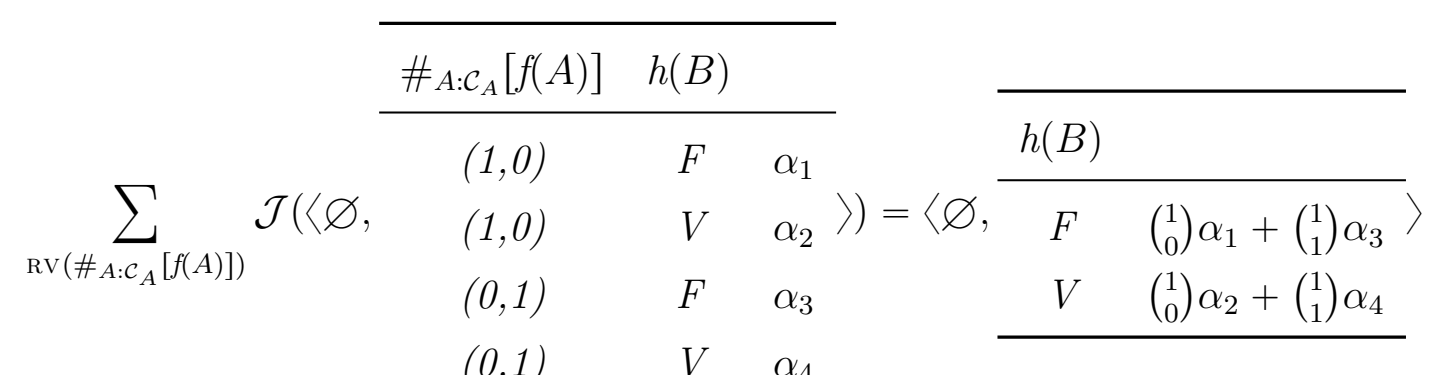

$\operatorname{Seja}\left|\mathcal{D}(A): \mathcal{C}_{A}\right|=2:$

$$
\begin{aligned}
& \begin{array}{ccc}
\hline \#_{A: \mathcal{C}_{A}}[f(A)] & h(B) & \\
\hline(2,0) & F & \alpha_{1}
\end{array}
\end{aligned}
$$

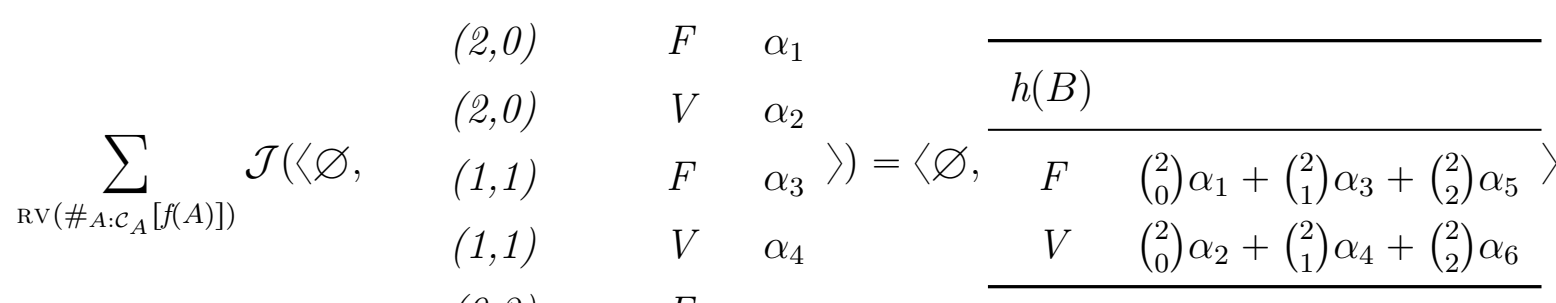

$$
\begin{aligned}
& (0,2) \quad F \quad \alpha_{5} \\
& (0,2) \quad V \quad \alpha_{6}
\end{aligned}
$$

$\operatorname{Seja}\left|\mathcal{D}(A): \mathcal{C}_{A}\right|=n:$

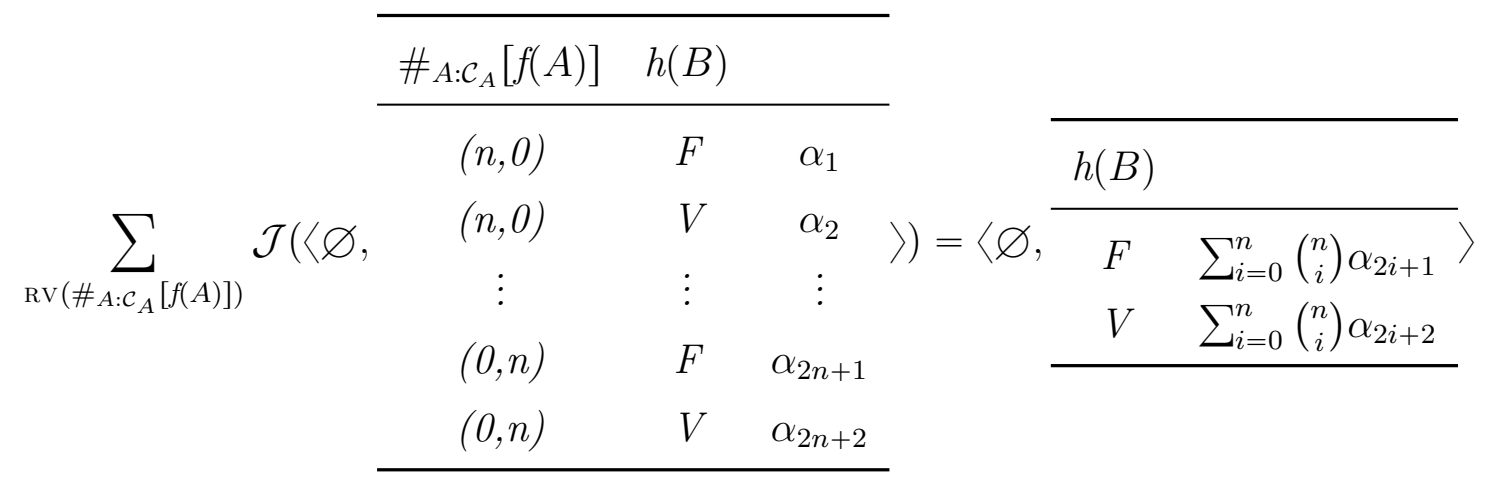

A Proposição 2 não se aplica a parfactors de agregação, pois estes não possuem a mesma estrutura de um parfactor na forma padrão. Como será explicado a seguir, em alguns casos é possível eliminar um variável aleatória parametrizada de um parfactor de agregação de maneira lifted sem a necessidade de convertê-lo para parfactors na forma padrão. 
Para tornar a exposição mais didática, um cenário básico será explicado e fatores complicadores serão adicionados ao longo da seção.

Considere um parfactor de agregação $\left\langle\mathcal{C}, p, c, V, \mathcal{F}_{p}, \otimes, A\right\rangle$. Assuma que o conjunto de variáveis de contexto $V$ é vazio. A relação entre as variáveis $p$ e $c$ é dada por $c=\bigotimes_{a \in \mathcal{D}(A): \mathcal{C}_{A}} \mathrm{p}(\ldots, a, \ldots)$. Note que, devido às propriedades comutativa e associativa, a variável $c$ é um "produtório" das variáveis aleatórias representadas por $p$ proposicionalizadas na variável lógica extra $A$. Este produtório é análogo ao cálculo de um número real $x$ elevado a um inteiro positivo $n$.

O algoritmo trivial para calcular $x^{n}$ consiste em iniciar com o valor de $x$ e multiplicá-lo $n-1$ vezes por ele mesmo. Entretanto, é possível obter o mesmo resultado utilizando menos multiplicações. Suponha por exemplo que se deseja calcular $x^{8}$. Este número pode ser obtido calculando o quadrado dos resultados parciais: $x^{8}=\left(\left(x^{2}\right)^{2}\right)^{2}$. Este procedimento utilizou apenas 3 multiplicações, enquanto que o método trivial necessitaria 7 multiplicações. A mesma ideia pode ser aplicada para qualquer $n$ inteiro positivo. Trata-se de um método bastante antigo, datado de 200 A.C ${ }^{3}$. O Algoritmo 2 ilustra o procedimento.

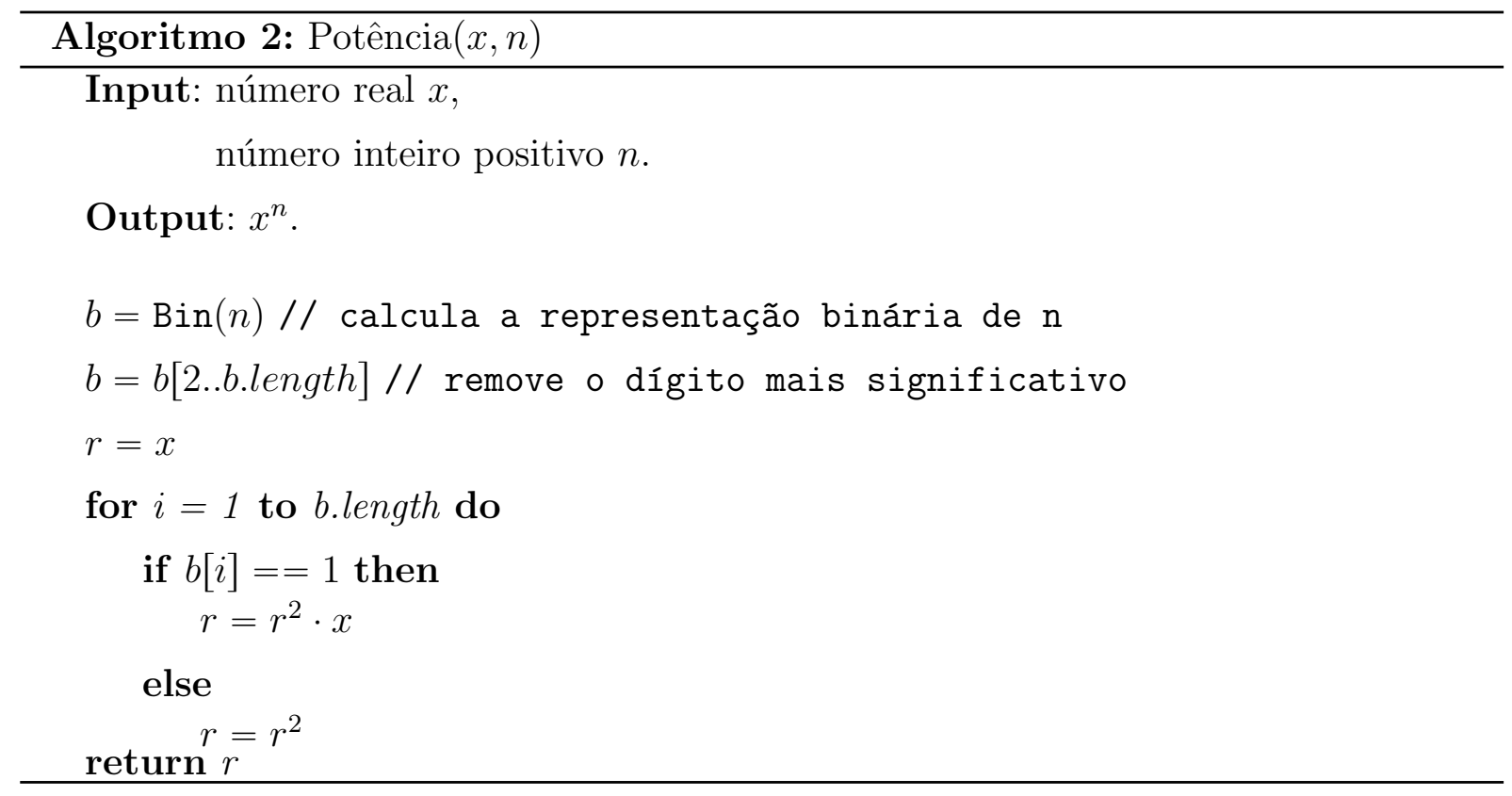

Exemplo 26. Utilizando o algoritmo 2, o cálculo de $x^{23}$ leva aos seguintes resultados intermediários: $x^{2}, x^{4}, x^{5}, x^{10}, x^{11}, x^{22}, x^{23}$. Note que o número 23 escrito na base 2 se torna 10111.

O Algoritmo 2 pode ser usado como base para eliminar a variável $p$ do parfactor $\langle\mathcal{C}$, $\left.p, c, V, \mathcal{F}_{p}, \otimes, A\right\rangle$. A eliminação da variável $p$ pode ser feita da seguinte maneira:

\footnotetext{
${ }^{3}$ PINGALA. Chandas Shastra.
} 
1. Proposicionalizar a variável lógica $A$;

2. Multiplicar todos os fatores resultantes que envolvem variáveis do tipo $\mathrm{p}(\ldots, a, \ldots)$, $a \in\left|\mathcal{D}(A): \mathcal{C}_{A}\right|$

3. Eliminar as variáveis $\mathrm{p}(\ldots, a, \ldots)$ do fator resultante.

O passo 2 envolve a multiplicação de vários fatores iguais. Para $n=\left|\mathcal{D}(A): \mathcal{C}_{A}\right|$, seja $b=b_{1} \ldots b_{m}$ a representação binária de $n$ sem o dígito binário mais à esquerda (isto é, se $n=6$ então $b=01)$. Seja $\left\{\mathcal{F}_{m}\right\}$ uma sequência de fatores definida recursivamente como

$$
\begin{aligned}
& \mathcal{F}_{0}=\mathcal{F}_{p} \\
& \mathcal{F}_{k}= \begin{cases}\sum \mathcal{F}_{k-1} \odot \mathcal{F}_{k-1} & \text { se } b_{k}=0 \\
\sum \sum \mathcal{F} \odot \mathcal{F}_{k-1} \odot \mathcal{F}_{k-1} & \text { se } b_{k}=1\end{cases}
\end{aligned}
$$

O operador $\sum$ indica a eliminação de um variável aleatória parametrizada do conjunto $\left\{p(\ldots, a, \ldots): a \in \mathcal{D}(A): \mathcal{C}_{A}\right\}$. Cada $\mathcal{F}_{k}$ é um fator $\mathcal{F}_{k}: \operatorname{RANGE}(p(\ldots, a, \ldots)) \rightarrow \mathbb{R}$ mas não é importante em qual indivíduo $a$ o fator está definido, pois todo $p(\ldots, a, \ldots)$ terá sido eliminado ao calcular o último termo da sequência. A ordem de eliminação é arbitrária pois o operador $\otimes$ é comutativo e associativo.

A sequência $\left\{\mathcal{F}_{m}\right\}$ será usada como base para a seguinte

Proposição 3. (KISYNSKI, 2010) Seja $g_{A}=\left\langle\mathcal{C}, p, c, V, \mathcal{F}_{p}, \otimes, A\right\rangle$ um parfactor de agregação pertencente a um conjunto de parfactors $G$. Suponha que:

- O conjunto de variáveis de contexto $V$ seja vazio;

- O conjunto de restrições $\mathcal{C}$ esteja na forma normal;

- $\operatorname{PARAM}(c)=\operatorname{PARAM}(p) \backslash\{A\}$;

- Nenhum outro parfactor de G possua variáveis aleatórias do conjunto RV $(p)$.

Então

$$
\sum_{\operatorname{Rv}(p)} g_{A}=\left\langle\mathcal{C} \backslash \mathcal{C}_{A},\{c\}, \mathcal{F}_{m}\right\rangle
$$

A Proposição 3 não contempla o caso em que a variável $c$ possui uma variável lógica que não existe em $p$. Para aplicar a Proposição 3 seria necessário eliminar a variável lógica 
extra de $c$ utilizando contagem, mas isto criaria uma fórmula de contagem que possui um domínio incompatível com o domínio de $p$.

Defina o fator $\mathcal{F}_{\#}: \operatorname{RANGE}\left(\#_{E: \mathcal{C}_{E}}[c]\right) \rightarrow \mathbb{R}$ obtido a partir do último termo da sequência $\left\{\mathcal{F}_{m}\right\}$, cujo valor para cada histograma $h$ de $\operatorname{RANGE}\left(\#_{E: \mathcal{C}_{E}}[c]\right)$ é dado por

$$
\mathcal{F}_{\#}(h)=\left\{\begin{array}{l}
\mathcal{F}_{m}(x), \text { se } \exists x \in \mathrm{RANGE}(c): h(x)=\left|\mathcal{D}(E): \mathcal{C}_{E}\right| \\
0, \text { caso contrário }
\end{array}\right.
$$

A partir da definição de $\mathcal{F}_{\#}$ estabelece-se a seguinte

Proposição 4. Seja $g_{A}=\left\langle\mathcal{C}, p, c, V, \mathcal{F}_{p}, \otimes, A\right\rangle$ um parfactor de agregação pertencente a um conjunto de parfactors $G$. Suponha que:

- O conjunto de variáveis de contexto $V$ seja vazio;

- O conjunto de restrições $\mathcal{C}$ esteja na forma normal;

- PARAM $(c) \backslash\{E\}=$ PARA $\mathrm{M}(p) \backslash\{A\}$, em que E é a variável lógica extra de $c$;

- Nenhum outro parfactor de $G$ possui variáveis aleatórias do conjunto RV $(p)$.

Então

$$
\sum_{\operatorname{Rv}(p)} g_{A}=\left\langle\mathcal{C} \backslash \mathcal{C}_{E},\left\{\# E_{E: \mathcal{C}_{E}}[c]\right\}, \mathcal{F}_{\#}\right\rangle
$$

O caso para parfactors de agregação com variáveis de contexto é tratado em seguida. Em geral, as Proposições 3 e 4 podem ser aplicadas a parfactors de agregação com variáveis de contexto bastando repetir os cálculos para cada valoração das variáveis de contexto. No entanto, existe uma condição adicional a ser verificada nas variáveis de contexto.

A nova condição é necessária porque, se uma variável de contexto de $V$ envolver a variável lógica $A$, a definição da sequência $\left\{\mathcal{F}_{m}\right\}$ se torna inválida. De fato, a definição da sequência impõe uma proposicionalização implícita da variável lógica $A$ na variável aleatória parametrizada $p$. Quando a variável $A$ também pertence a uma ou mais variáveis de contexto, é necessário proposicionalizar $A$ nestas variáveis, o que acarreta em um aumento exponencial do tamanho dos fatores $\mathcal{F}_{k}$. Esta particularidade não foi tratada por Kisynski (2010) em sua formalização do algoritmo AC-F OVE e será tratada com mais detalhes no Capítulo 5, por se tratar da contribuição teórica mais relevante deste trabalho. A Proposição 3 é reescrita a seguir para que ela possa ser aplicada a parfactors de agregação com variáveis de contexto. 
Proposição 5. (KISYNSKI, 2010) Seja $g_{A}=\left\langle\mathcal{C}, p, c, V, \mathcal{F}_{p}, \otimes, A\right\rangle$ um parfactor de agregação pertencente a um conjunto de parfactors $G$. Suponha que:

- $\mathcal{C}$ esteja na forma normal;

- $\operatorname{PARAM}(c)=\operatorname{PARAM}(p) \backslash\{A\}$;

- Nenhum outro parfactor de G possua variáveis aleatórias do conjunto RV $(p)$;

- Nenhuma variável de contexto em $V$ contenha a variável lógica A.

Então

$$
\sum_{\operatorname{Rv}(p)} g_{A}=\left\langle\mathcal{C} \backslash \mathcal{C}_{A},\{c\} \cup V, \mathcal{F}_{m}\right\rangle
$$

\subsubsection{Multiplicação}

A multiplicação de parfactors permite combinar parfactors que possuem variáveis aleatórias parametrizadas equivalentes. O objetivo da multiplicação de parfactors é satisfazer a Condição 2 da Proposição 2.

A Proposição 6 foi adaptada de Kisynski (2010) e deriva do trabalho de Milch et al. (2008) e Braz, Amir e Roth (2007).

Proposição 6. Seja $G$ um conjunto de parfactors com $|G| \geqslant 2$ e sejam $g_{1}=\left\langle\mathcal{C}_{1}, \mathcal{V}_{1}, \mathcal{F}_{1}\right\rangle$ e $g_{2}=\left\langle\mathcal{C}_{2}, \mathcal{V}_{2}, \mathcal{F}_{2}\right\rangle$ parfactors de $G$. Suponha que:

1. Os conjuntos de variáveis aleatórias representadas por variáveis aleatórias parametrizadas de $g_{1}$ e $g_{2}$ são iguais ou disjuntos;

2. Para todo par de variáveis aleatórias parametrizadas equivalentes $(f, h)$ de $\mathcal{V}_{1} \times \mathcal{V}_{2}$, tem-se PARAM $(f)=$ PARAM $(h)$ e o conjunto das outras variáveis lógicas de $g_{1} e ́$ disjunto do conjunto das outras variáveis lógicas de $g_{2}$.

Defina um parfactor intermediário $\bar{g}=\left\langle\mathcal{C}_{1} \cup \mathcal{C}_{2}, \mathcal{V}_{1} \cup \mathcal{V}_{2}, \mathcal{F}_{1} \odot \mathcal{F}_{2}\right\rangle$ e os expoentes de correção $r_{1}=\left|g_{1}\right| /|g|$ e $r_{2}=\left|g_{2}\right| /|g|$. Seja $g_{m}=\left\langle\mathcal{C}_{1} \cup \mathcal{C}_{2}, \mathcal{V}_{1} \cup \mathcal{V}_{2}, \mathcal{F}_{1}^{r_{1}} \odot \mathcal{F}_{2}^{r_{2}}\right\rangle$. Então

$$
\mathcal{J}(G)=\mathcal{J}\left(G \backslash\left\{g_{1}, g_{2}\right\} \cup\left\{g_{m}\right\}\right)
$$

O Exemplo 27 foi extraído de Kisynski (2010). 
Exemplo 27. Considere as variáveis lógicas $A, B$ e $C$ tais que $|\mathcal{D}(A)|=|\mathcal{D}(B)|=n$ $e|\mathcal{D}(C)|=m$. Sejam $f(A, B), h(B)$ e e $(C)$ variáveis aleatórias parametrizadas com $\operatorname{RANGE}(f)=\operatorname{RANGE}(h)=\{\mathrm{V}, \mathrm{F}\}$ e RANGE $(e)=\{$ verde, laranja, vermelho $\}$. Considere o seguinte conjunto de parfactors:

\begin{tabular}{|c|c|c|c|}
\hline \multirow{5}{*}{$G=\{\langle\{A \neq B\}$} & $f(A, B)$ & $h(B)$ & \\
\hline & $F$ & $F$ & $\alpha_{1}$ \\
\hline & $F$ & $V$ & $\alpha_{2}>$ \\
\hline & $V$ & $F$ & $\alpha_{3}$ \\
\hline & $V$ & $V$ & $\alpha_{4}$ \\
\hline & $e(C)$ & $h(B)$ & \\
\hline & verde & $F$ & $\beta_{1}$ \\
\hline & verde & $V$ & $\beta_{2}$ \\
\hline$\langle\varnothing$ & laranja & $F$ & $\left.\beta_{3}\right\rangle$ \\
\hline & laranja & $V$ & $\beta_{4}$ \\
\hline & vermelho & $F$ & $\beta_{5}$ \\
\hline & vermelho & $V$ & $\beta_{6}$ \\
\hline
\end{tabular}

Note que as condições para multiplicação são verdadeiras para os parfactors $g_{1}$ e $g_{2}$ :

- $\operatorname{RV}(f):\{A \neq B\} \cap \operatorname{RV}(e): \varnothing=\varnothing$;

- $\operatorname{RV}(f):\{A \neq B\} \cap \operatorname{RV}(h): \varnothing=\varnothing$;

- $\operatorname{RV}(h):\{A \neq B\} \cap \operatorname{RV}(e): \varnothing=\varnothing$;

- $\operatorname{RV}(h):\{A \neq B\}=\operatorname{RV}(h): \varnothing$. Neste caso, em $g_{1}$ resta o conjunto de variáveis lógicas $\{A\}$ e em $g_{2}$ resta o conjunto $\{C\}$, que são evidentemente disjuntos.

$O$ parfactor $g_{1}$ representa $n(n-1)$ fatores e o parfactor $g_{2}$ representa $n m$ fatores; o produto 
representará $n m(n-1)$ fatores. O produto $g_{m}=g_{1} \odot g_{2}$ será dado por

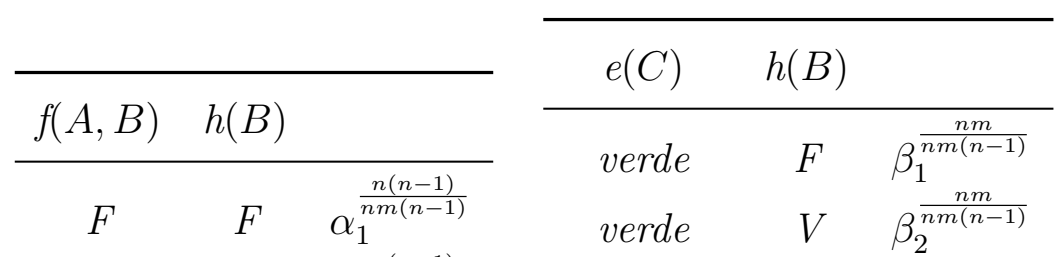

$$
\begin{aligned}
& g_{m}=\left\langle\{A \neq B\}, \quad F \quad V \quad \alpha_{2}^{\frac{n(n-1)}{n m(n-1)}} \odot \text { laranja } \quad F \quad \beta_{3}^{\frac{n m}{n m(n-1)}}\right\rangle \\
& V \quad F \quad \alpha_{3}^{\frac{n(n-1)}{n m(n-1)}} \quad \text { laranja } \quad V \quad \beta_{4}^{\frac{n m}{n m(n-1)}}
\end{aligned}
$$

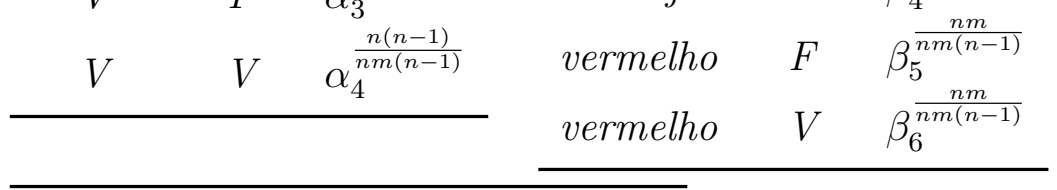

$$
\begin{aligned}
& \begin{array}{cccc}
\hline f(A, B) & e(C) & h(B) & \\
\hline F & \text { verde } & F & \alpha_{1}^{\frac{1}{m}} \beta_{1}^{\frac{1}{n-1}}
\end{array} \\
& \text { F verde } \quad \text { V } \quad \alpha_{2}^{\frac{1}{m}} \beta_{2}^{\frac{1}{n-1}} \\
& \text { F laranja } \quad F \quad \alpha_{1}^{\frac{1}{m}} \beta_{3}^{\frac{1}{n-1}} \\
& \text { F laranja } \quad V \quad \alpha_{2}^{\frac{1}{m}} \beta_{4}^{\frac{1}{n-1}} \\
& \text { F vermelho } \quad F \quad \alpha_{1}^{\frac{1}{m}} \beta_{5}^{\frac{1}{n-1}} \\
& =\left\langle\{A \neq B\}, \quad F \quad \text { vermelho } \quad V \quad \alpha_{2}^{\frac{1}{m}} \beta_{6}^{\frac{1}{n-1}}\right\rangle \\
& \text { V verde } \quad F \quad \alpha_{3}^{\frac{1}{m}} \beta_{1}^{\frac{1}{n-1}} \\
& V \quad \text { verde } \quad V \quad \alpha_{4}^{\frac{1}{m}} \beta_{2}^{\frac{1}{n-1}} \\
& V \quad \text { laranja } \quad F \quad \alpha_{3}^{\frac{1}{m}} \beta_{3}^{\frac{1}{n-1}} \\
& V \quad \text { laranja } \quad V \quad \alpha_{4}^{\frac{1}{m}} \beta_{4}^{\frac{1}{n-1}} \\
& V \quad \text { vermelho } F \quad \alpha_{3}^{\frac{1}{m}} \beta_{5}^{\frac{1}{n-1}} \\
& \text { V vermelho } \quad V \quad \alpha_{4}^{\frac{1}{m}} \beta_{6}^{\frac{1}{n-1}}
\end{aligned}
$$

Pela Proposição $6, \mathcal{J}(G)=\mathcal{J}\left(g_{m}\right)$.

Em determinadas circunstâncias, também é possível multiplicar um parfactor na forma padrão por um parfactor de agregação. A Proposição 7 formaliza a multiplicação para parfactors de agregação (KISYNSKI, 2010).

Proposição 7. Sejam $g_{A}=\left\langle\mathcal{C}, p, c, V, \mathcal{F}_{p}, \otimes, A\right\rangle$ e $g=\langle\mathcal{C},\{p\} \cup V, \mathcal{F}\rangle$ dois parfactors de um conjunto de parfactors $G$. O produto de $g_{A}$ por $g$ é dado por

$$
g_{m}=\left\langle\mathcal{C}, p, c, V, \mathcal{F}_{p} \odot \mathcal{F}, \otimes, A\right\rangle .
$$

Tem-se ainda

$$
\mathcal{J}(G)=\mathcal{J}\left(G \backslash\left\{g_{A}, g\right\} \cup\left\{g_{m}\right\}\right)
$$




\subsubsection{Divisão e proposicionalização}

Uma das condições para realizar multiplicação entre parfactors requer que os conjuntos de variáveis aleatórias representados por cada uma das variáveis aleatórias parametrizadas sejam iguais ou disjuntos. Esta condição pode ser satisfeita através da operação de divisão, que "quebra" uma variável aleatória parametrizada em dois conjuntos disjuntos.

A divisão de um parfactor $g$ na substituição $\{X / t\}$ gera dois parfactors: um parfactor $g[X / t]$ (denominado resultado) que é obtido aplicando-se a substituição $\{X / t\}$ no parfactor $g$ e um parfactor $g^{\prime}$ denominado resíduo.

A operação de divisão será tratada a seguir de maneira separada para parfactors na forma padrão e para parfactors de agregação. A Proposição 8 trata da divisão em parfactors na forma padrão (MILCH et al., 2008; KISYNSKI; POOLE, 2009). As Proposições 9 e 10 tratam da divisão em parfactors de agregação (KISYNSKI; POOLE, 2009).

Proposição 8. Seja $g=\langle\mathcal{C}, \mathcal{V}, \mathcal{F}\rangle$ um parfactor na forma padrão pertencente a um conjunto de parfactors $G$. Seja $\{X / t\}$ uma substituição tal que:

1. A variável lógica $X$ esteja presente em g;

2. O termo $t$ não apareça em nenhuma restrição envolvendo $X$, isto é, $t \notin E_{X}^{\mathcal{C}}$;

3. Se o termo t é uma constante, então $t \in \mathcal{D}(X)$;

4. Se o termo t é uma variável lógica, então $\mathcal{D}(t)=\mathcal{D}(X)$ e t está presente em $g$.

Seja $g[X / t]$ o parfactor resultante da aplicação da substituição $\{X / t\}$ em $g$ e $g^{\prime}=$ $\langle\mathcal{C} \cup\{X \neq t\}, \mathcal{V}, \mathcal{F}\rangle$. Então

$$
\mathcal{J}(G)=\mathcal{J}\left(G \backslash g \cup\left\{g[X / t], g^{\prime}\right\}\right)
$$

Exemplo 28. Considere as variáveis lógicas $A$ e $B \operatorname{com} \mathcal{D}(A)=\mathcal{D}(B)=\left\{x_{1}, x_{2}, \ldots\right.$, $\left.x_{n}\right\}$ e as variáveis aleatórias parametrizadas $f(A, B)$ e $h(B)$, ambas com domínio booleano. Considere o seguinte parfactor

$$
\begin{array}{cccc}
\cline { 2 - 3 }\langle f(A, B) & h(B) & \\
\cline { 2 - 3 }\left\langle\left\{A \neq x_{1}\right\},\right. & F & F & \alpha_{1} \\
V & V & \alpha_{2} \\
V & F & \alpha_{3} \\
V & V & \alpha_{4}
\end{array}
$$


Note que o parfactor $g_{1}$ representa $n(n-1)$ fatores. Ao dividir $g_{1}$ na substituição $\left\{B / x_{2}\right\}$, obtém-se dois parfactors:

\begin{tabular}{|c|c|c|c|}
\hline \multirow{5}{*}{$\left\langle\left\{A \neq x_{2}\right\}\right.}$, & $f\left(A, x_{2}\right)$ & $h\left(x_{2}\right)$ & \\
\hline & $F$ & $F$ & $\alpha_{1}$ \\
\hline & $F$ & $V$ & $\alpha_{2}$ \\
\hline & $V$ & $F$ & $\alpha_{3}$ \\
\hline & $V$ & $V$ & $\alpha_{4}$ \\
\hline \multirow{5}{*}{$\left\langle\left\{A \neq x_{1}, B \neq x_{2}\right\}\right.$} & $f(A, B)$ & $h(B)$ & \\
\hline & $F$ & $F$ & $\alpha_{1}$ \\
\hline & $F$ & $V$ & $\alpha_{2}$ \\
\hline & $V$ & $F$ & $\alpha_{3}$ \\
\hline & $V$ & $V$ & $\alpha_{4}$ \\
\hline
\end{tabular}

$O$ parfactor $g_{2}$ representa $n-1$ fatores e o parfactor $g_{3}$ representa $(n-1)^{2}$ fatores. Pela Proposição 8, $\mathcal{J}\left(g_{1}\right)=\mathcal{J}\left(g_{2}, g_{3}\right)$.

Proposição 9. Considere um parfactor de agregação $g_{A}=\left\langle\mathcal{C}, p, c, V, \mathcal{F}_{p}, \otimes, A\right\rangle$ pertencente a um conjunto de parfactors $G$. Seja $\{X / t\}$ uma substituição tal que:

1. A variável lógica $X$ seja diferente da variável lógica extra A;

2. A variável lógica $X$ seja parâmetro da variável aleatória parametrizada c;

3. O termo t seja diferente da variável lógica extra A;

4. Se o termo t é uma constante, então $t \in \mathcal{D}(X)$;

5. Se o termo t é uma variável lógica, então $t \in \mathrm{PARAM}(c)$;

6. A restrição $X \neq t$ não exista no conjunto $\mathcal{C}$.

Seja

- $g_{A}[X / t]=\left\langle\mathcal{C}[X / t], p[X / t], c[X / t], V[X / t], \mathcal{F}_{p}, \otimes, A\right\rangle$

- $g_{A}{ }^{\prime}=\left\langle\mathcal{C} \cup\{X \neq t\}, p, c, V, \mathcal{F}_{p}, \otimes, A\right\rangle$

Então

$$
\mathcal{J}(G)=\mathcal{J}\left(G \backslash g_{A} \cup\left\{g_{A}[X / t], g_{A}{ }^{\prime}\right\}\right)
$$


Exemplo 29. Considere as variáveis lógicas $A$ e $B$ com $\mathcal{D}(A)=\mathcal{D}(B)=\left\{x_{1}, x_{2}, \ldots\right.$, $\left.x_{n}\right\}$ e as variáveis aleatórias parametrizadas $f(A, B)$ e $h(B)$, ambas com dominio booleano. Considere o parfactor de agregação

$$
\left\langle\varnothing, p(A, B), c(B),\{v(A), u(B)\}, \mathcal{F}_{p}, \otimes, A\right\rangle
$$

Ao dividir $g_{1}$ na substituição $\left\{B / x_{1}\right\}$, obtém-se dois parfactors:

$$
\begin{array}{r}
\left\langle\varnothing, p\left(A, x_{1}\right), c\left(x_{1}\right),\left\{v(A), u\left(x_{1}\right)\right\}, \mathcal{F}_{p}, \otimes, A\right\rangle \\
\left\langle\left\{B \neq x_{1}\right\}, p(A, B), c(B),\{v(A), u(B)\}, \mathcal{F}_{p}, \otimes, A\right\rangle
\end{array}
$$

Pela Proposição 9, $\mathcal{J}\left(g_{1}\right)=\mathcal{J}\left(g_{2}, g_{3}\right)$.

Exemplo 30. Considere as variáveis lógicas $A$ e $B \operatorname{com} \mathcal{D}(A)=\mathcal{D}(B)=\left\{x_{1}, x_{2}, \ldots\right.$, $\left.x_{n}\right\}$ e as variáveis aleatórias parametrizadas $f(A, B)$ e $h(B)$, ambas com dominio booleano. Considere o parfactor de agregação

$$
\left\langle\left\{A \neq x_{2}, B \neq x_{2}\right\}, p(A, B), c(B),\{v(A), u(B)\}, \mathcal{F}_{p}, \otimes, A\right\rangle
$$

Ao dividir $g_{1}$ na substituição $\left\{B / x_{1}\right\}$, obtém-se dois parfactors:

$$
\begin{array}{r}
\left\langle\left\{A \neq x_{2}\right\}, p\left(A, x_{1}\right), c\left(x_{1}\right),\left\{v(A), u\left(x_{1}\right)\right\}, \mathcal{F}_{p}, \otimes, A\right\rangle \\
\left\langle\left\{A \neq x_{2}, B \neq x_{1}, B \neq x_{2}\right\}, p(A, B), c(B),\{v(A), u(B)\}, \mathcal{F}_{p}, \otimes, A\right\rangle
\end{array}
$$

Pela Proposição 9, $\mathcal{J}\left(g_{1}\right)=\mathcal{J}\left(g_{2}, g_{3}\right)$.

Proposição 10. Considere um parfactor de agregação $g_{A}=\left\langle\mathcal{C}, p, c, V, \mathcal{F}_{p}, \otimes, A\right\rangle$ pertencente a um conjunto de parfactors $G$. Seja $\{A / t\}$ uma substituição tal que:

1. Se o termo t é uma constante, então $t \in \mathcal{D}(A)$;

2. Se o termo $t$ é uma variável lógica, então $t \in \mathrm{PARAM}(p) \backslash\{A\}$;

3. $A$ restrição $A \neq t$ não existe no conjunto $\mathcal{C}$.

Defina uma variável aleatória parametrizada auxiliar $c^{\prime}$ equivalente a c. Seja $\mathcal{V}=\left\{f_{1}\right.$, $\left.\ldots, f_{m}\right\}, r_{p}=|\operatorname{RV}(p[A / a])|, a \in \mathcal{D}(A): \mathcal{C}$ e $r_{c}=|\operatorname{RV}(c): \mathcal{C}|$. Defina um fator $\mathcal{F}_{c}:$ $\operatorname{RANGE}(p) \times \operatorname{RANGE}\left(f_{1}\right) \times \cdots \times \operatorname{RANGE}\left(f_{m}\right) \times \operatorname{RANGE}(c) \times \operatorname{RANGE}\left(c^{\prime}\right) \rightarrow \mathbb{R}$ tal que, para cada possivel atribuição de valor $\mathbf{v}$ às variáveis de $\mathcal{F}_{c}$, tem-se

$$
\mathcal{F}_{c}(\mathbf{v})= \begin{cases}\mathcal{F}_{p}^{\frac{r_{p}}{r_{c}}}(\mathbf{v}), & \text { se } \mathbf{v}(c)=\mathbf{v}(p) \otimes \mathbf{v}\left(c^{\prime}\right) \\ 0, & \text { caso contrário }\end{cases}
$$


Seja

- $g_{A}[A / t]=\left\langle\mathcal{C}[A / t],\left\{p[A / t], f_{1}[A / t], \ldots, f_{m}[A / t], c^{\prime}, c\right\}, \mathcal{F}_{c}\right\rangle$

- $g_{A}{ }^{\prime}=\left\langle\mathcal{C} \cup\{A \neq t\}, p, c^{\prime}, V, \mathcal{F}_{p}, \otimes, A\right\rangle$

Então

$$
\mathcal{J}(G)=\sum_{\operatorname{Rv}\left(c^{\prime}\right)} \mathcal{J}\left(G \backslash g_{A} \cup\left\{g_{A}[A / t], g_{A}{ }^{\prime}\right\}\right)
$$

Exemplo 31. Considere as variáveis lógicas $A$ e $B \operatorname{com} \mathcal{D}(A)=\mathcal{D}(B)=\left\{x_{1}, x_{2}, \ldots\right.$, $\left.x_{n}\right\}$ e as variáveis aleatórias parametrizadas $f(A, B)$ e $h(B)$, ambas com domínio booleano. Considere o parfactor de agregação

$$
\left\langle\left\{A \neq x_{2}, B \neq x_{2}\right\}, p(A, B), c(B),\{v(A), u(B)\}, \mathbb{1}, \mathrm{OR}, A\right\rangle
$$

Ao dividir $g_{1}$ na substituição $\left\{A / x_{1}\right\}$, obtém-se dois parfactors:

$$
\begin{array}{r}
\left\langle\left\{A \neq x_{1}, A \neq x_{2}, B \neq x_{2}\right\}, p(A, B), c^{\prime}(B),\{v(A), u(B)\}, \mathbb{1}, \text { o R }, A\right\rangle \\
\left\langle\left\{B \neq x_{2}\right\},\left\{p\left(x_{1}, B\right), v\left(x_{1}\right), u(B), c^{\prime}(B), c(B)\right\}, \mathcal{F}_{c}\right\rangle
\end{array}
$$


em que

\begin{tabular}{|c|c|c|c|c|c|}
\hline$p\left(x_{1}, B\right)$ & $v\left(x_{1}\right)$ & $u(B)$ & $c^{\prime}(B)$ & $c(B)$ & \\
\hline$F$ & $F$ & $F$ & $F$ & $F$ & 1 \\
\hline$F$ & $F$ & $F$ & $F$ & $V$ & 0 \\
\hline$F$ & $F$ & $F$ & $V$ & $F$ & 0 \\
\hline$F$ & $F$ & $F$ & $V$ & $V$ & 1 \\
\hline$F$ & $F$ & $V$ & $F$ & $F$ & 0 \\
\hline$F$ & $F$ & $V$ & $F$ & $V$ & 1 \\
\hline$F$ & $F$ & $V$ & $V$ & $F$ & 0 \\
\hline$F$ & $F$ & $V$ & $V$ & $V$ & 1 \\
\hline$F$ & $V$ & $F$ & $F$ & $F$ & 0 \\
\hline$F$ & $V$ & $F$ & $F$ & $V$ & 1 \\
\hline$F$ & $V$ & $F$ & $V$ & $F$ & 0 \\
\hline$F$ & $V$ & $F$ & $V$ & $V$ & 1 \\
\hline$F$ & $V$ & $V$ & $F$ & $F$ & 0 \\
\hline$F$ & $V$ & $V$ & $F$ & $V$ & 1 \\
\hline$F$ & $V$ & $V$ & $V$ & $F$ & 0 \\
\hline$F$ & $V$ & $V$ & $V$ & $V$ & 1 \\
\hline$V$ & $F$ & $F$ & $F$ & $F$ & 0 \\
\hline$V$ & $F$ & $F$ & $F$ & $V$ & 1 \\
\hline$V$ & $F$ & $F$ & $V$ & $F$ & 0 \\
\hline$V$ & $F$ & $F$ & $V$ & $V$ & 1 \\
\hline$V$ & $F$ & $V$ & $F$ & $F$ & 0 \\
\hline$V$ & $F$ & $V$ & $F$ & $V$ & 1 \\
\hline$V$ & $F$ & $V$ & $V$ & $F$ & 0 \\
\hline$V$ & $F$ & $V$ & $V$ & $V$ & 1 \\
\hline$V$ & $V$ & $F$ & $F$ & $F$ & 0 \\
\hline$V$ & $V$ & $F$ & $F$ & $V$ & 1 \\
\hline$V$ & $V$ & $F$ & $V$ & $F$ & 0 \\
\hline$V$ & $V$ & $F$ & $V$ & $V$ & 1 \\
\hline$V$ & $V$ & $V$ & $F$ & $F$ & 0 \\
\hline$V$ & $V$ & $V$ & $F$ & $V$ & 1 \\
\hline$V$ & $V$ & $V$ & $V$ & $F$ & 0 \\
\hline$V$ & $V$ & $V$ & $V$ & $V$ & 1 \\
\hline
\end{tabular}

Pela Proposição 10, $\mathcal{J}\left(g_{1}\right)=\sum_{\mathrm{Rv}\left(c^{\prime}\right)} \mathcal{J}\left(g_{2}, g_{3}\right)$. 
A proposicionalização de uma variável lógica $X$ presente em um parfactor $g=\langle\mathcal{C}, \mathcal{V}, \mathcal{F}\rangle$ é uma operação que consiste em substituir $g$ por um conjunto de parfactors $g[X / t]$, em que cada termo $t$ é uma constante do conjunto $\mathcal{D}(X): \mathcal{C}$. A proposicionalização de $g \mathrm{em}$ $X$ também pode ser obtida pela divisão de $g$ em todas as substituições da forma $\{X / t\}$ $\operatorname{com} t \in \mathcal{D}(X): \mathcal{C}$.

Exemplo 32. Considere a variável lógica $A \operatorname{com} \mathcal{D}(A)=\left\{x_{1}, x_{2}, \ldots, x_{n}\right\}$ e a variável aleatória parametrizada $f(A)$ com dominio booleano. Considere o parfactor $g=\left\langle\left\{A \neq x_{1}\right\}\right.$, $\{f(A)\}, \mathcal{F}\rangle$. Note que g representa $n-1$ fatores. Ao proposicionalizar a variável lógica $A$ em $g$, obtém-se $n-1$ parfactors:

$$
\left\langle\varnothing,\left\{f\left(x_{2}\right)\right\}, \mathcal{F}\right\rangle,\left\langle\varnothing,\left\{f\left(x_{3}\right)\right\}, \mathcal{F}\right\rangle, \ldots,\left\langle\varnothing,\left\{f\left(x_{n}\right)\right\}, \mathcal{F}\right\rangle .
$$

\subsubsection{Expansão}

A expansão é uma operação análoga à divisão aplicada a fórmulas de contagem. Esta operação consiste em "dividir" uma fórmula de contagem em dois conjuntos disjuntos. Por exemplo, a fórmula de contagem no fator $\mathcal{F}\left(\#_{X}[\mathrm{f}(X)]\right)$ pode ser expandida num indivíduo $x_{1}$ de $\mathcal{D}(X)$ resultando em um fator $\mathcal{F}^{\prime}\left(\#_{X: X \neq x_{1}}[\mathrm{f}(X)], \mathrm{f}\left(x_{1}\right)\right)$. A expansão de uma fórmula de contagem gera um novo parfactor que representa o mesmo conjunto de fatores do parfactor não expandido.

A Proposição 11 formaliza a expansão de fórmula de contagem em um parfactor e foi proposta por Milch et al. (2008).

Proposição 11. Considere o parfactor $g=\langle\mathcal{C}, \mathcal{V}, \mathcal{F}\rangle$ pertencente a um conjunto de parfactors $G$. Seja $\gamma=\#_{A: \mathcal{C}_{A}}[f]$ uma fórmula de contagem presente no conjunto $\mathcal{V}$ e $t$ um termo tal que:

1. O termo $t$ não aparece em nenhuma restrição de $\mathcal{C}_{A}$;

2. Para cada variável lógica $Y$ em restrições de $\mathcal{C}_{A}$ (isto é, para cada $Y \in E_{A}^{\mathcal{C}_{A}}$ ), existe uma restrição $Y \neq t$ em $\mathcal{C}$.

Defina uma fórmula de contagem $\gamma_{e}=\#_{A: \mathcal{C}_{A} \cup\{A \neq t\}}[f]$ e um parfactor $g_{e}=\left\langle\mathcal{C}, \mathcal{V}_{e}, \mathcal{F}_{e}\right\rangle$ tal que:

- $\mathcal{V}_{e}=\mathcal{V} \backslash\{\gamma\} \cup\left\{f[A / t], \gamma_{e}\right\}$; 
- $\mathcal{F}_{e}$ é um fator $\mathcal{F}_{e}: \times_{y \in \mathcal{V}_{e}} \operatorname{RANGE}(y) \rightarrow \mathbb{R}$. Para cada possível atribuição de valores $\mathbf{v}$ às variáveis de $\mathcal{V} \backslash\left\{\#_{A: \mathcal{C}_{A}}[f]\right\}$ e para todo $x \in \operatorname{RANGE}(f), h_{e} \in \operatorname{RANGE}\left(\gamma_{e}\right) e$ $h \in \operatorname{range}(\gamma)$,

$$
\mathcal{F}_{e}\left(\mathbf{v}, h_{e}, x\right)=\mathcal{F}(\mathbf{v}, h)
$$

em que o histograma $h$ é obtido somando-se 1 ao elemento do histograma $h_{e}$ que corresponde ao valor ${ }^{4}$ de $x$.

Então

$$
\mathcal{J}(G)=\mathcal{J}\left(G \backslash\{g\} \cup\left\{g_{e}\right\}\right)
$$

No Exemplo 33, a expansão de uma fórmula de contagem é feita passo a passo para tornar o conceito mais claro. Ele foi extraído e adaptado de Kisynski (2010).

Exemplo 33. Considere o seguinte parfactor:

$$
\begin{array}{ccc}
\hline \#_{A:\{A \neq B\}}[f(A)] & h(B) & \\
\hline\left(\#_{\mathrm{F}}=n-1, \#_{\mathrm{V}}=0\right) & F & \alpha_{1} \\
\left(\#_{\mathrm{F}}=n-1, \#_{\mathrm{V}}=0\right) & V & \alpha_{2} \\
\left(\#_{\mathrm{F}}=n-2, \#_{\mathrm{V}}=1\right) & F & \left.\alpha_{3}\right\rangle \\
\left(\#_{\mathrm{F}}=n-2, \#_{\mathrm{V}}=1\right) & V & \alpha_{4} \\
\vdots & \vdots & \vdots \\
\left(\#_{\mathrm{F}}=0, \#_{\mathrm{V}}=n-1\right) & F & \alpha_{2 n-1} \\
\left(\#_{\mathrm{F}}=0, \#_{\mathrm{V}}=n-1\right) & V & \alpha_{2 n} \\
\hline
\end{array}
$$

em que $\mathcal{D}(A)=\mathcal{D}(B)=\left\{x_{1}, x_{2}, \ldots, x_{n}\right\}$, RANGE $(f)=\operatorname{RANGE}(h)=\{\mathrm{F}, \mathrm{V}\} e$ $\alpha_{1}, \ldots, \alpha_{2 n} \in \mathbb{R}$. O parfactor $g_{1}$ representa $n$ fatores, cada um com tamanho $2 n$. Suponha que se deseja expandir a fórmula de contagem $\#_{A:\{A \neq B\}}[f(A)]$ na constante $x_{1}$.

Primeiramente, "extrai-se" $x_{1}$ da fórmula de contagem pela adição de uma restrição (isto é, remove-se o elemento $f\left(x_{1}\right)$ da contagem):

$$
\#_{A:\{A \neq B\}}[f(A)] \equiv \#_{A:\left\{A \neq B, A \neq x_{1}\right\}}[f(A)], f\left(x_{1}\right)
$$

Em seguida, a variável aleatória parametrizada extraída $f\left(x_{1}\right)$ é adicionada ao fator

\footnotetext{
${ }^{4}$ Nesta expressão, v representa o conjunto de valores das variáveis aleatórias parametrizadas em comum entre $\mathcal{F}_{e}$ e $\mathcal{F}$.
} 
original. Assim, a linha

\begin{tabular}{ccc}
\hline$\#_{A:\{A \neq B\}}[f(A)]$ & $h(B)$ & \\
\hline$\vdots$ & $\vdots$ & $\vdots$ \\
$\left(\#_{\mathrm{F}}=n-1, \#_{\mathrm{V}}=0\right)$ & $F$ & $\alpha_{1}$ \\
$\vdots$ & $\vdots$ & $\vdots$ \\
\hline
\end{tabular}

do fator original se decompõe em duas linhas:

\begin{tabular}{cccc}
\hline$\#_{A:\left\{A \neq B, A \neq x_{1}\right\}}[f(A)]$ & $f\left(x_{1}\right)$ & $h(B)$ & \\
\hline$\vdots$ & $\vdots$ & $\vdots$ & $\vdots$ \\
$\left(\#_{\mathrm{F}}=n-2, \#_{\mathrm{V}}=0\right)$ & $F$ & $F$ & $\alpha_{1}$ \\
$\left(\#_{\mathrm{F}}=n-2, \#_{\mathrm{V}}=0\right)$ & $V$ & $F$ & $\alpha_{3}$ \\
$\vdots$ & $\vdots$ & $\vdots$ & $\vdots$ \\
\hline
\end{tabular}

Note que

$$
\begin{aligned}
& \left(\#_{\mathrm{F}}=n-2, \#_{\mathrm{V}}=0\right) \text { combinado com } \mathrm{F} \text { resulta }\left(\#_{\mathrm{F}}=n-1, \#_{\mathrm{V}}=0\right) \\
& \left(\#_{\mathrm{F}}=n-2, \#_{\mathrm{V}}=0\right) \text { combinado com } \mathrm{V} \text { resulta }\left(\#_{\mathrm{F}}=n-2, \#_{\mathrm{V}}=1\right) .
\end{aligned}
$$

Repete-se a operação de decomposição de linhas para todas as linhas do fator original. A expansão de $\#_{A:\{A \neq B\}}[f(A)]$ em $x_{1}$ resulta no parfactor

$$
\begin{array}{cccc}
\hline \#_{A:\left\{A \neq B, A \neq x_{1}\right\}}[f(A)] & f\left(x_{1}\right) & h(B) & \\
\hline\left(\#_{\mathrm{F}}=n-2, \#_{\mathrm{V}}=0\right) & F & F & \alpha_{1} \\
\left(\#_{\mathrm{F}}=n-2, \#_{\mathrm{V}}=0\right) & F & V & \alpha_{2} \\
\left(\#_{\mathrm{F}}=n-2, \#_{\mathrm{V}}=0\right) & V & F & \alpha_{3} \\
\left(\#_{\mathrm{F}}=n-2, \#_{\mathrm{V}}=0\right) & V & V & \alpha_{4} \\
\left(\#_{\mathrm{F}}=n-3, \#_{\mathrm{V}}=1\right) & F & F & \alpha_{3} \\
\left(\#_{\mathrm{F}}=n-3, \#_{\mathrm{V}}=1\right) & F & V & \alpha_{4} \\
\left(\#_{\mathrm{F}}=n-3, \#_{\mathrm{V}}=1\right) & V & F & \alpha_{5} \\
\left(\#_{\mathrm{F}}=n-3, \#_{\mathrm{V}}=1\right) & V & V & \alpha_{6} \\
\vdots & \vdots & \vdots & \vdots \\
\left(\#_{\mathrm{F}}=0, \#_{\mathrm{V}}=n-2\right) & V & F & \alpha_{2 n-1} \\
\left(\#_{\mathrm{F}}=0, \#_{\mathrm{V}}=n-2\right) & V & V & \alpha_{2 n} \\
\hline
\end{array}
$$

O parfactor $g_{2}$ representa $n$ fatores. Neste exemplo, cada $\alpha_{i}$ aparece 2 vezes, com exceção de 
$\alpha_{1}, \alpha_{2}, \alpha_{2 n-1}$ e $\alpha_{2 n}$. Assim, cada fator representado por $g_{2}$ tem tamanho $2 \cdot 2 n-4=4(n-1)$. Pela Proposição $11, \mathcal{J}\left(g_{1}\right)=\mathcal{J}\left(g_{2}\right)$.

\subsubsection{Conversão}

Parfactors de agregação podem ser convertidos para parfactors na forma padrão (KISYNSKI; POOLE, 2009) com a utilização de fórmulas de contagem. Esta operação é utilizada quando um parfactor de agregação não pode ser combinado com outro por meio da Multiplicação, nem pode ter a variável parametrizada pai eliminada pelas Proposições 4 ou 5. A Proposição 12, elaborada por Kisynski (2010), ilustra como esta conversão é feita.

Proposição 12. Seja $g_{A}=\left\langle\mathcal{C}, p, c, V, \mathcal{F}_{p}, \otimes, A\right\rangle$ um parfactor de agregação na forma normal, com $\mathcal{V}=\left\{f_{1}, \ldots, f_{m}\right\}$. Seja $\mathcal{C}_{A}$ o subconjunto de restrições de $\mathcal{C}$ que envolvem a variável lógica $A$. Seja $\gamma=\#_{A: \mathcal{C}_{A}}[p]$ uma fórmula de contagem e $\mathcal{F}_{\#}: \operatorname{RANGE}(\gamma) \times$ $\operatorname{RANGE}\left(f_{1}\right) \times \cdots \times \operatorname{RANGE}\left(f_{m}\right) \times \operatorname{RANGE}(c) \rightarrow \mathbb{R}$ um fator tal que, para toda atribuição de valores às variáveis do conjunto $\mathcal{V} \cup\{c, \gamma\}$, tem-se $e^{5}$

$$
\mathcal{F}_{\#}= \begin{cases}1 & \text { se } \bigotimes_{x \in \mathrm{RANGE}_{(p)}} \bigotimes_{(\mathbf{v}(\gamma))(x)} x=\mathbf{v}(c) \\ 0 & \text { caso contrário }\end{cases}
$$

Defina dois parfactors $g_{1}=\left\langle\mathcal{C},\{p\} \cup \mathcal{V}, \mathcal{F}_{p}\right\rangle$ e $g_{2}=\left\langle\mathcal{C} \backslash \mathcal{C}_{A},\{\gamma, c\} \cup \mathcal{V}, \mathcal{F}_{\#}\right\rangle$. Então

$$
\mathcal{J}\left(g_{A}\right)=\mathcal{J}\left(g_{1}, g_{2}\right)
$$

Exemplo 34. Considere o parfactor de agregação $g_{A}=\left\langle\left\{A \neq a_{1}\right\}, p(A), c(),\{h()\}, \mathbb{1}, \mathrm{OR}\right.$, A) e suponha que $\mathcal{D}(A)=\left\{a_{1}, a_{2}, \ldots, a_{n}\right\}$. Pela Proposição 12, a conversão de $g_{A}$ para parfactors na forma padrão resulta em dois parfactors:

$$
\begin{aligned}
& \mathcal{J}\left(g_{A}\right)=\mathcal{J}\left(\left\langle A \neq a_{1},\{p(A), h()\}, \mathbb{1}\right\rangle\right. \\
&\left.\left\langle\varnothing,\left\{\#_{A:\left\{A \neq a_{1}\right\}}[p], c(), h()\right\}, \mathcal{F}_{\#}\right\rangle\right)
\end{aligned}
$$

O fator $\mathcal{F}_{\#}$ é uma função $f: \operatorname{RANGE}\left(\#_{A:\left\{A \neq a_{1}\right\}}[p]\right) \times \operatorname{RANGE}(c) \times \operatorname{RANGE}(h) \rightarrow \mathbb{R}$. Dada uma atribuição de valores $\mathbf{v}$ às variáveis $\#_{A:\left\{A \neq a_{1}\right\}}[p]$, c e h, o valor de $\mathcal{F}_{\#}(\mathbf{v})$ é obtido pela "expansão" do histograma $h=\mathbf{v}\left(\#_{A:\left\{A \neq a_{1}\right\}}[p]\right)$ e pela aplicação do operador

\footnotetext{
${ }^{5}$ Note que $\mathbf{v}(\gamma)$ denota um histograma do domínio da fórmula de contagem $\gamma$, e portanto $(\mathbf{v}(\gamma))(x)$ denota a contagem do elemento $x$ neste histograma.
} 
de agregação. Assim, se $\mathbf{v}=\left(\left(\#_{\mathrm{F}}=n-2, \#_{\mathrm{V}}=1\right), \mathrm{V}, \mathrm{V}\right)$, tem-se

$$
\underset{x \in\{\mathrm{F}, \mathrm{V}\}}{\mathrm{OR}} \underset{h(x)}{\mathrm{OR}} x=\underbrace{\mathrm{F} \text { OR } \ldots \text { OR F }}_{n-2 \text { vezes }} \text { OR } \mathrm{V}=\mathrm{V}=\mathbf{v}(c)
$$

e portanto $\mathcal{F}_{\#}(\mathbf{v})=1$. Ao fazer a mesma operação para todas as possíveis atribuições de valores às variáveis $\#_{A:\left\{A \neq a_{1}\right\}}[p]$, c e h, obtém-se a representação tabular de $\mathcal{F}_{\#}$ :

\begin{tabular}{rlll}
\hline$\#_{A:\left\{A \neq a_{1}\right\}}[p]$ & $c()$ & $h()$ & \\
\hline$\left(\#_{\mathrm{F}}=n-1, \#_{\mathrm{V}}=0\right)$ & $F$ & $F$ & 1 \\
$\left(\#_{\mathrm{F}}=n-1, \#_{\mathrm{V}}=0\right)$ & $F$ & $V$ & 1 \\
$\left(\#_{\mathrm{F}}=n-1, \#_{\mathrm{V}}=0\right)$ & $V$ & $F$ & 0 \\
$\left(\#_{\mathrm{F}}=n-1, \#_{\mathrm{V}}=0\right)$ & $V$ & $V$ & 0 \\
$\left(\#_{\mathrm{F}}=n-2, \#_{\mathrm{V}}=1\right)$ & $F$ & $F$ & 0 \\
$\left(\#_{\mathrm{F}}=n-2, \#_{\mathrm{V}}=1\right)$ & $F$ & $V$ & 0 \\
$\left(\#_{\mathrm{F}}=n-2, \#_{\mathrm{V}}=1\right)$ & $V$ & $F$ & 1 \\
$\left(\#_{\mathrm{F}}=n-2, \#_{\mathrm{V}}=1\right)$ & $V$ & $V$ & 1 \\
$\vdots$ & $\vdots$ & $\vdots$ & $\vdots$ \\
$\left(\#_{\mathrm{F}}=0, \#_{\mathrm{V}}=n-1\right)$ & $F$ & $F$ & 0 \\
$\left(\#_{\mathrm{F}}=0, \#_{\mathrm{V}}=n-1\right)$ & $F$ & $V$ & 0 \\
$\left(\#_{\mathrm{F}}=0, \#_{\mathrm{V}}=n-1\right)$ & $V$ & $F$ & 1 \\
$\left(\#_{\mathrm{F}}=0, \#_{\mathrm{V}}=n-1\right)$ & $V$ & $V$ & 1 \\
\hline
\end{tabular}

\subsubsection{Contagem}

Uma das condições para eliminar uma variável aleatória parametrizada de um parfactor é que todas as variáveis lógicas que aparecem no parfactor apareçam também nesta variável aleatória parametrizada. Em algumas situações, esta condição pode ser atingida por meio da operação de contagem, que consiste em eliminar uma variável lógica de um parfactor.

A Proposição 13 foi estabelecida por Milch et al. (2008).

Proposição 13. Considere um parfactor na forma normal $g=\langle\mathcal{C}, \mathcal{V}, \mathcal{F}\rangle$ pertencente a um conjunto de parfactors $G$. Seja A uma variável lógica que aparece em uma única variável aleatória parametrizada $f$ de $g$. Seja $\mathcal{C}_{A}$ o subconjunto de restrições de $\mathcal{C}$ que envolvem a variável lógica $A$.

Defina uma fórmula de contagem $\gamma=\#_{A: \mathcal{C}_{A}}[f]$ e um parfactor $g_{c}=\left\langle\mathcal{C}_{c}, \mathcal{V}_{c}, \mathcal{F}_{c}\right\rangle$ tal que: 
- $\mathcal{C}_{c}=\mathcal{C} \backslash \mathcal{C}_{A}$

- $\mathcal{V}_{c}=\mathcal{V} \backslash\{f\} \cup\{\gamma\}$

- Para todas as atribuições de valores $\mathbf{v}$ às variáveis de $\mathcal{V} \backslash\{f\}$ e todo histograma $h \in \operatorname{RANGE}(\gamma)$,

$$
\mathcal{F}_{e}(\mathbf{v}, h)=\prod_{x \in \operatorname{RANGE}(f)} \mathcal{F}(\mathbf{v}, x)^{h(x)}
$$

Então

$$
\mathcal{J}(G)=\mathcal{J}\left(G \backslash\{g\} \cup\left\{g_{c}\right\}\right)
$$

Os Exemplo 35 ilustra a operação de contagem para diversas situações.

Exemplo 35. Seja $A$ uma variável lógica tal que $\mathcal{D}(A)=\left\{a_{1}, \ldots, a_{k}\right\}$. No que se segue, o símbolo $\equiv$ indica a equivalência dos parfactors após a eliminação da variável lógica $A$ através de contagem.

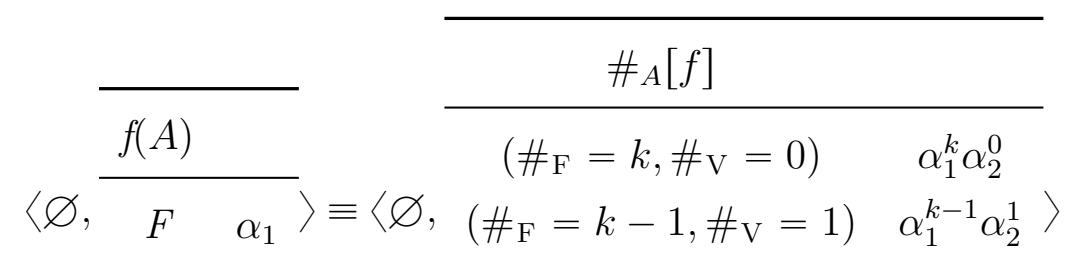

$$
\begin{aligned}
& \begin{array}{lll}
V & \alpha_{2} & \vdots
\end{array} \\
& \left(\#_{\mathrm{F}}=0, \#_{\mathrm{V}}=k\right) \quad \alpha_{1}^{0} \alpha_{2}^{k} \\
& \left\langle\left\{\begin{array}{ll}
\frac{\#_{A: A \neq \alpha_{1}}[f]}{f(A)} & \\
\left\langle\left\{A \neq \alpha_{1}\right\}, \frac{\left.\#_{\mathrm{F}}=k-1, \#_{\mathrm{V}}=0\right)}{F^{2} \quad \alpha_{1}}\right\rangle \equiv\langle\varnothing, & \alpha_{1}^{k-1} \alpha_{2}^{0} \\
\left(\#_{\mathrm{F}}=k-2, \#_{\mathrm{V}}=1\right) & \left.\alpha_{1}^{k-2} \alpha_{2}^{1}\right\rangle
\end{array}\right.\right. \\
& \begin{array}{llll}
V & \alpha_{2} & \vdots & \vdots \\
\hline
\end{array} \\
& \left(\#_{\mathrm{F}}=0, \#_{\mathrm{V}}=k-1\right) \quad \alpha_{1}^{0} \alpha_{2}^{k-1}
\end{aligned}
$$

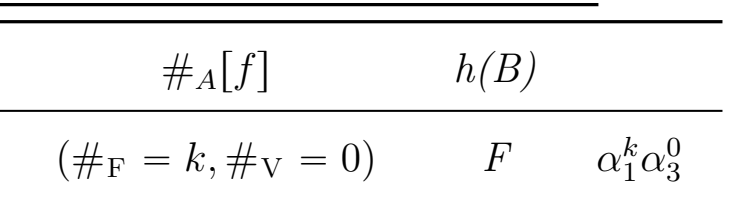

$$
\begin{aligned}
& \begin{array}{ccc}
\hline f(A) & h(B) & \\
\hline F & F & \alpha_{1}
\end{array} \\
& \left(\#_{\mathrm{F}}=k, \#_{\mathrm{V}}=0\right) \quad V \quad \alpha_{2}^{k} \alpha_{4}^{0}
\end{aligned}
$$

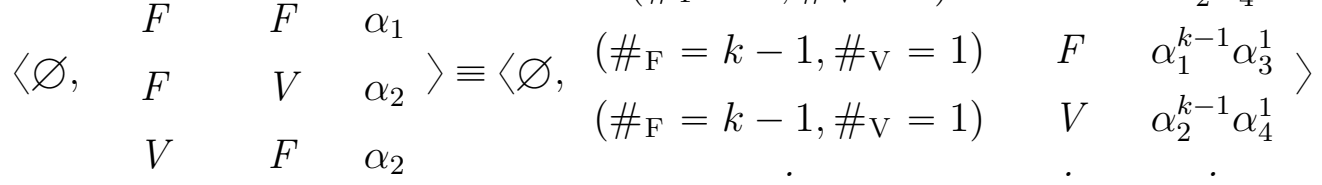

$$
\begin{aligned}
& \begin{array}{cccccc}
V & V & \alpha_{3} & \vdots & \vdots & \vdots \\
& & & \left(\#_{\mathrm{F}}=0, \#_{\mathrm{V}}=k\right) & F & \alpha_{1}^{0} \alpha_{3}^{k} \\
& & \left(\#_{\mathrm{F}}=0, \#_{\mathrm{V}}=k\right) & V & \alpha_{2}^{0} \alpha_{4}^{k} \\
\hline
\end{array}
\end{aligned}
$$




\subsubsection{Unificação}

As operações de eliminação e multiplicação impõem condições que devem ser verificadas previamente. Uma destas condições diz respeito aos conjuntos de variáveis aleatórias representadas por variáveis aleatórias parametrizadas dos parfactors que devem ser iguais ou disjuntos. Verificar esta condição pela análise de todo o conjunto acabaria com o propósito da inferência em primeira ordem. Poole (2003) propôs uma maneira de verificar estas condições por meio de unificação (derivada da operação de mesmo nome para lógicas de primeira ordem). Milch et al. (2008) estendeu o conceito para fórmulas de contagem e Kisynski (2010) consolidou as definições.

Dados dois parfactors $g_{1}$ e $g_{2}$, o objetivo da unificação é encontrar a relação entre $\mathrm{f}\left(t_{1}^{1}\right.$, $\left.\ldots, t_{k}^{1}\right) \in g_{1}$ e $\mathrm{f}\left(t_{1}^{2}, \ldots, t_{k}^{2}\right) \in g_{2}$, isto é, a relação entre os conjuntos de variáveis aleatórias $\operatorname{RV}(f): \mathcal{C}_{1}$ e $\operatorname{RV}(f): \mathcal{C}_{2}$. A comparação só faz sentido se as duas variáveis aleatórias parametrizadas em questão possuem o mesmo functor e o mesmo número de termos, caso contrário elas representam conjuntos disjuntos de variáveis aleatórias. Quando existir uma intersecção não vazia entre os conjuntos e estes não forem iguais, deseja-se particionar os conjuntos de maneira a torná-los iguais ou disjuntos.

A unificação consiste em encontrar o unificador mais geral (MGU) entre os termos de duas variáveis aleatórias parametrizadas e, em seguida, realizar as divisões e expansões necessárias para tornar os conjuntos de variáveis aleatórias iguais ou disjuntos entre si. Somente a informação do tamanho dos conjuntos de variáveis aleatórias parametrizadas é usada para determinar quais serão as operações necessárias. Desse modo, é importante que o tamanho destes conjuntos permaneça fixo independentemente da substituição que é feita, o que é garantido pela condição (3.1.1) imposta na definição de parfactors. Para tornar a exposição mais didática, a operação de unificação será apresentada em etapas acompanhadas de exemplos de aplicação.

\subsubsection{Pré-processamento}

Algumas vezes o conjunto de restrições de um parfactor é suficientemente grande para limitar uma variável lógica a um indivíduo da população a ela atrelada. Por motivos que serão explicitados mais adiante, nestas situações a variável lógica será substituída pelo indivíduo. Para isso, utiliza-se o algoritmo AC-3(MACKWORTH, 1977), adaptado por Kisynski (2010). O pseudocódigo é exibido no Algoritmo 3. 


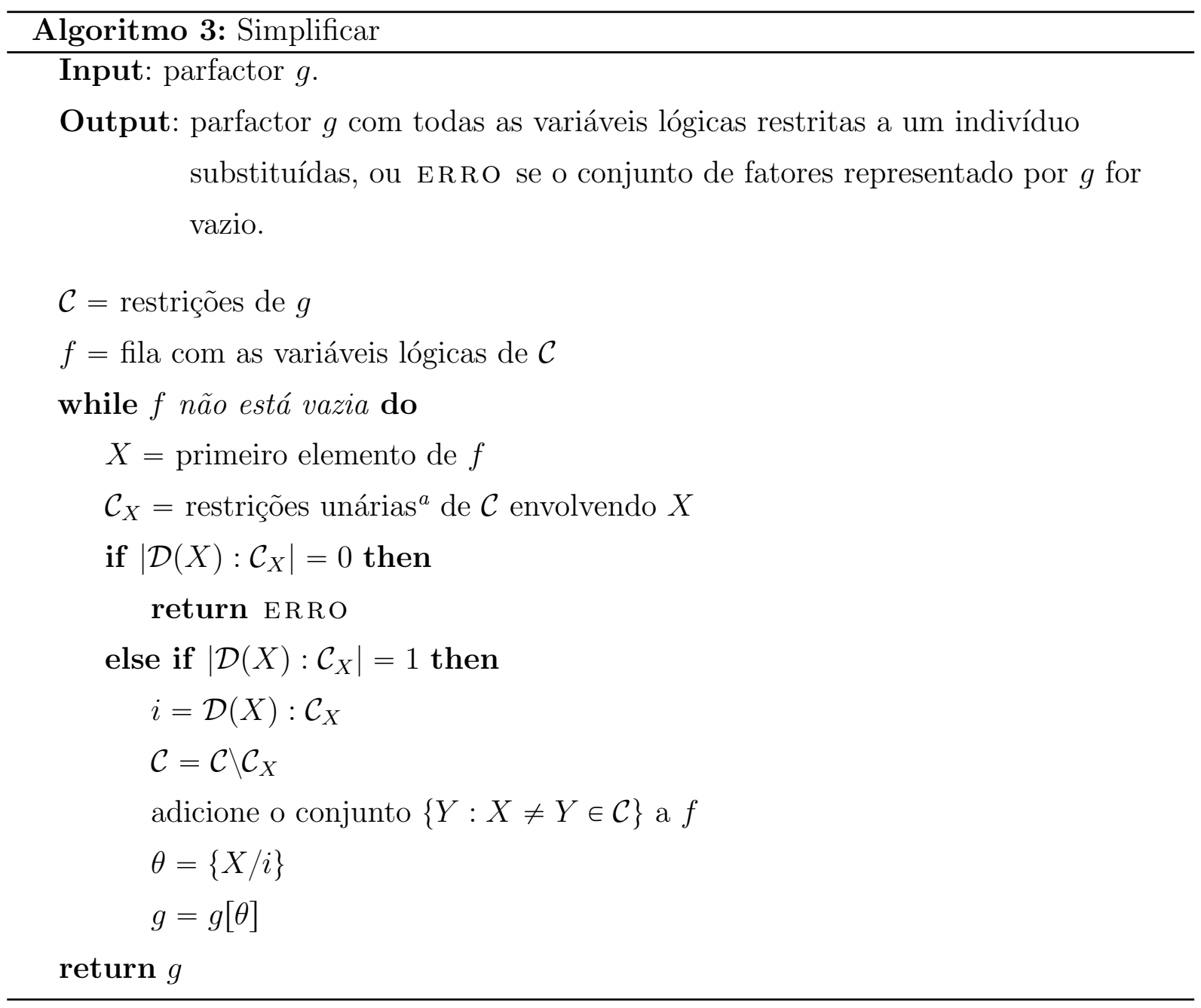

${ }^{a}$ Restrições da forma $X \neq t$ em que $X$ é variável lógica e $t$ é constante.

Exemplo 36. Considere o parfactor $g=\langle\mathcal{C}, \mathcal{V}, \mathcal{F}\rangle$ em que $\mathcal{C}=\left\{A \neq x_{1}, A \neq x_{2}, A \neq\right.$ $\left.B, B \neq x_{1}, B \neq C\right\}$ e $\mathcal{D}(A)=\mathcal{D}(B)=\mathcal{D}(C)=\left\{x_{1}, x_{2}, x_{3}\right\}$. O Algoritmo 3 detecta que a variável lógica $A$ está restrita ao indivíduo $x_{3}$. Após a substituição, o conjunto de restrições se torna $\mathcal{C}^{\prime}=\left\{B \neq x_{3}, B \neq x_{1}, B \neq C\right\}$. Note que agora a variável lógica $B$ está restrita ao indivíduo $x_{2}$. Após a substituição $\left\{B / x_{2}\right\}$, o conjunto de restrições se reduz a $\mathcal{C}^{\prime \prime}=\left\{C \neq x_{2}\right\}$. Ao final da simplificação, foi feita a substituição $\theta=\left\{A / x_{3}, B / x_{2}\right\}$ e o Algoritmo 3 retorna o parfactor $g[\theta]=\left\langle\mathcal{C}^{\prime \prime}, \mathcal{V}[\theta], \mathcal{F}\right\rangle$.

Como o escopo das variáveis lógicas se restringe ao parfactor em que estão inseridas, é necessário realizar um tratamento para evitar conflitos de nomes. Um variável lógica $X$ em um parfactor $g_{1}$ pode ter uma população atrelada diferente de uma outra variável lógica $X$ de um parfactor $g_{2}$. Para que a unificação funcione, é necessário distinguir estas variáveis lógicas. O pseudocódigo ilustrado pelo Algoritmo 4 realiza a renomeação das variáveis lógicas de um conjunto de parfactors. 


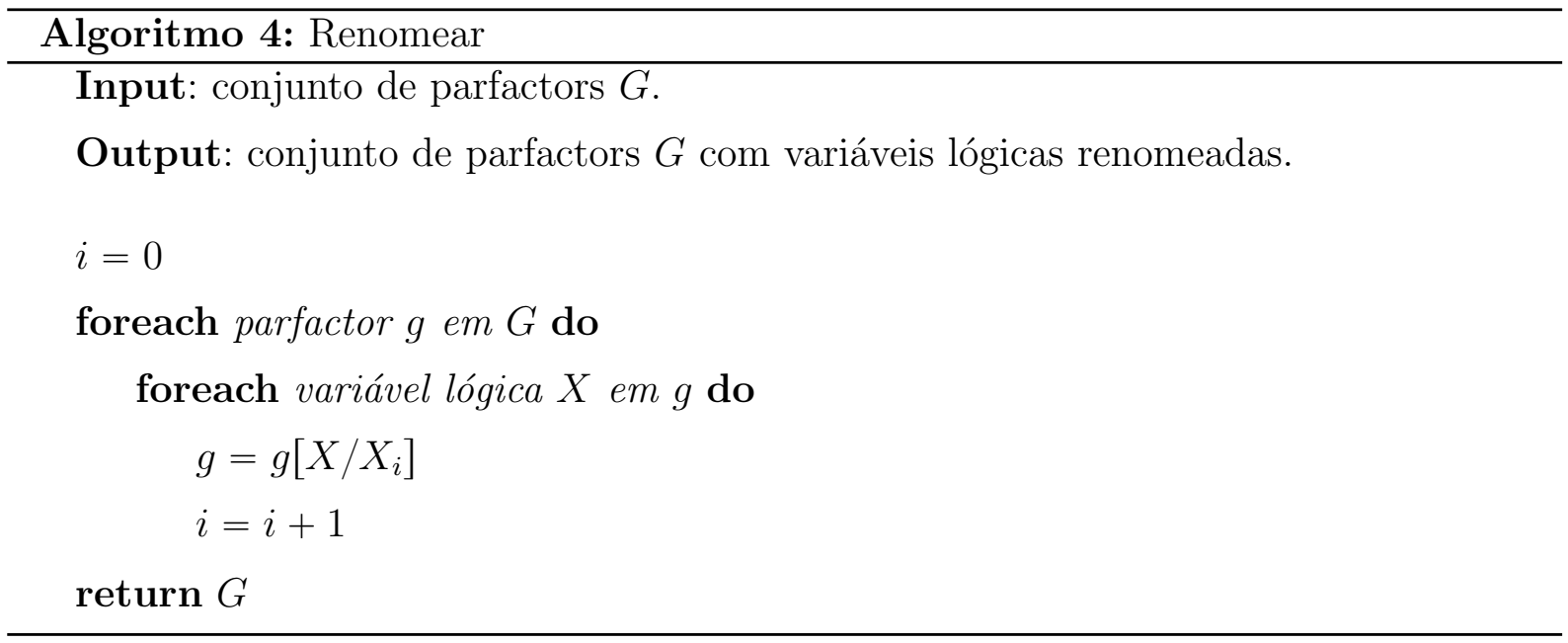

Exemplo 37. Considere o seguinte conjunto de parfactors:

$$
\begin{aligned}
G=\{ & \left\langle\left\{X \neq x_{1}\right\},\{f(X), h(X, Y)\}, \mathcal{F}_{1}\right\rangle \\
& \left.\left\langle\left\{X \neq Z, Y \neq y_{1}\right\},\{f(X), p(Y, Z)\}, \mathcal{F}_{2}\right\rangle\right\}
\end{aligned}
$$

Suponha que $\mathcal{D}(X)=\mathcal{D}(Y)=\left\{x_{1}, x_{2}, x_{3}\right\}$ em $g_{1}$ e $\mathcal{D}(X)=\mathcal{D}(Z)=\left\{x_{1}, x_{2}, x_{3}\right\}$ e $\mathcal{D}(Y)=$ $\left\{y_{1}, y_{2}, y_{3}\right\}$ em $g_{2}$. Note que a variável lógica $Y$ está atrelada a diferentes populações em $g_{1}$ e $g_{2}$. Utilizando o Algoritmo 4, obtém-se o seguinte conjunto de parfactors após a renomeação:

$$
\begin{aligned}
G=\{ & \left\langle\left\{X_{1} \neq x_{1}\right\},\left\{f\left(X_{1}\right), h\left(X_{1}, Y_{2}\right)\right\}, \mathcal{F}_{1}\right\rangle \\
& \left.\left\langle\left\{X_{3} \neq Z_{4}, Y_{5} \neq y_{1}\right\},\left\{f\left(X_{3}\right), p\left(Y_{5}, Z_{4}\right)\right\}, \mathcal{F}_{2}\right\rangle\right\}
\end{aligned}
$$

\subsubsection{Unificador mais geral}

O cálculo do MGU pode ser realizado após o pré-processamento detalhado nas seções anteriores. Para se obter o MGU, utiliza-se o algoritmo proposto por Sterling e Shapiro (1994) e adaptado por Kisynski (2010). O pseudocódigo é exibido no Algoritmo 5. 


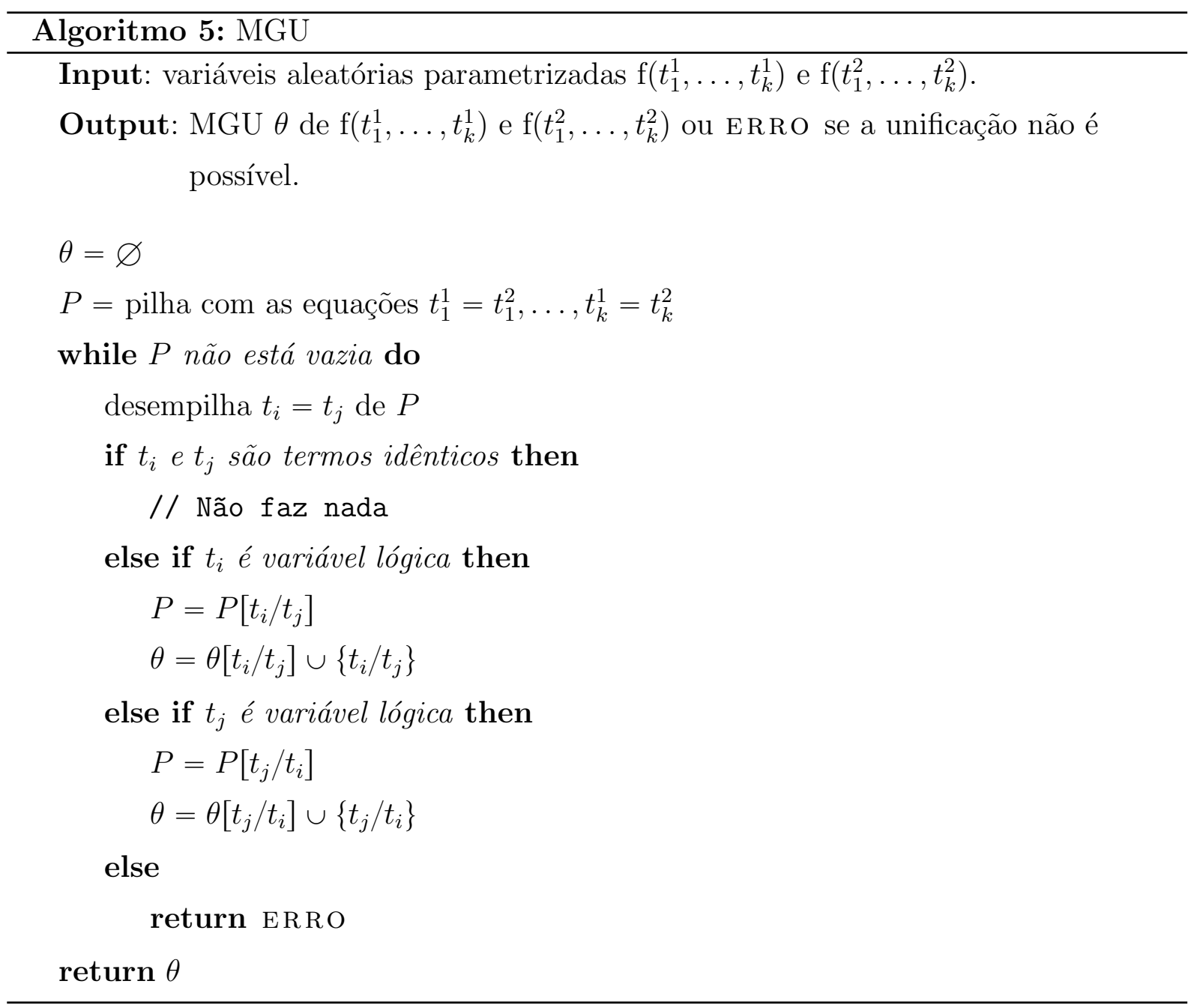

Exemplo 38. Considere as variáveis aleatórias parametrizadas $f\left(X, Y, z_{1}\right)$ e $f\left(x_{1}, W, Z\right)$. A aplicação do algoritmo 5 retorna o $M G U \theta=\left\{X / x_{1}, Y / W, Z / z_{1}\right\}$.

Após o cálculo do MGU, três situações podem ocorrer:

MGU é vazio. Ambas variáveis aleatórias parametrizadas não possuem variáveis lógicas, o que implica que cada uma representa a mesma variável aleatória. Note que isto é verdade pois todas as variáveis lógicas restritas a um indivíduo da população foram substituídas por este indivíduo.

O algoritmo retorna erro. As variáveis aleatórias parametrizadas não podem ser unificadas, e portanto representam conjuntos distintos de variáveis aleatórias. Isto é válido pois foi feita uma renomeação de variáveis lógicas de maneira que as variáveis lógicas presentes em uma variável aleatória parametrizada não ocorram na outra variável aleatória parametrizada ${ }^{6}$.

\footnotetext{
${ }^{6}$ Note que ERRO ocorre somente quando $t_{i}$ e $t_{j}$ são constantes e $t_{i} \neq t_{j}$.
} 
O MGU não é vazio. As variáveis aleatórias parametrizadas se unificam.

Para o caso em que o MGU não é vazio, é necessário verificar se ele é consistente com as restrições dos parfactors em questão. Uma substituição $\theta$ é consistente com um conjunto de restrições $\mathcal{C}$ quando $\mathcal{C}[\theta]$ contém apenas inequações válidas ou verdadeiras. $\mathrm{O}$ Algoritmo 6 ilustra o pseudocódigo para esta verificação (KISYNSKI, 2010).

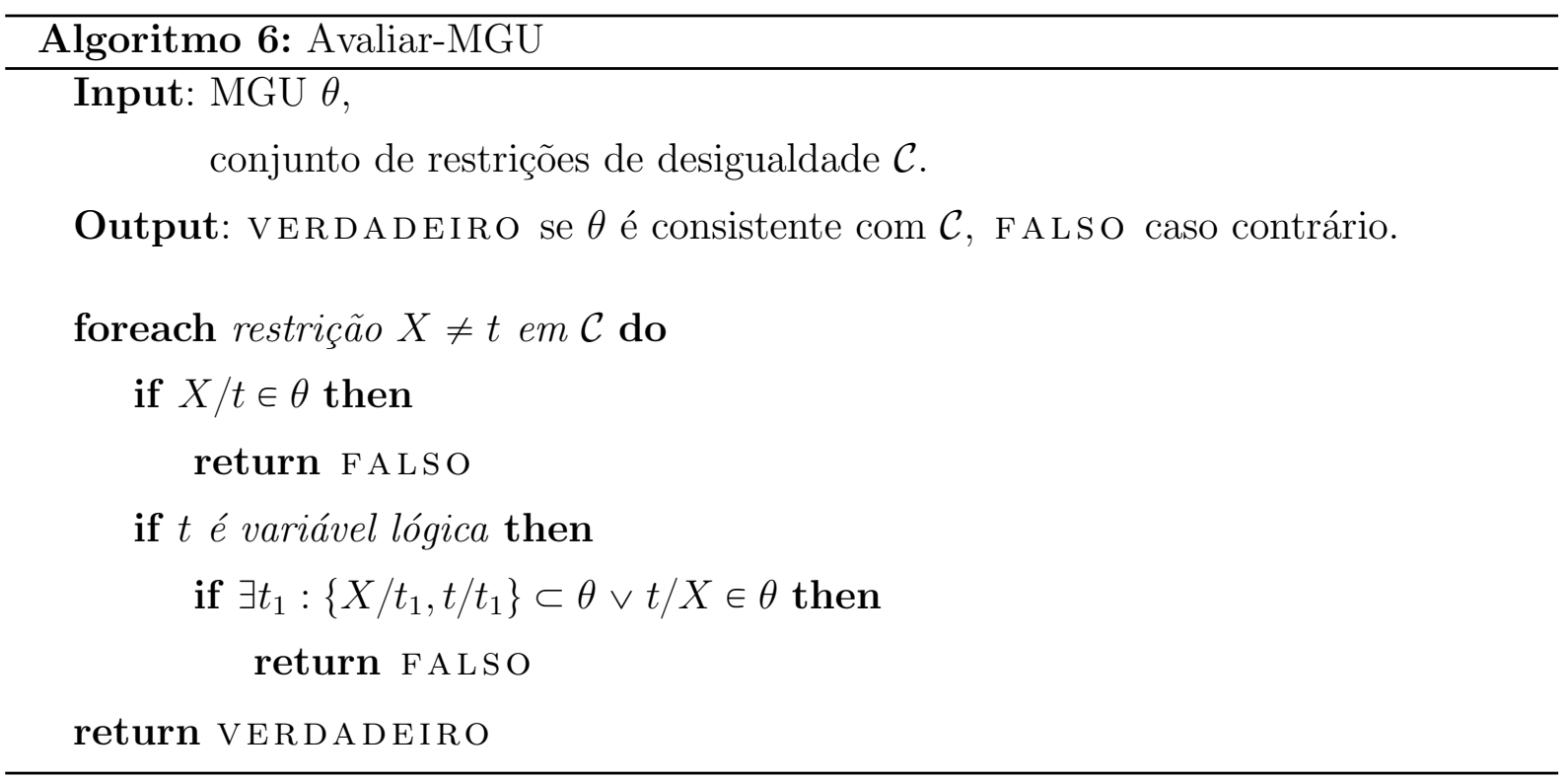

Exemplo 39. Considere que o cálculo de um $M G U$ resultou na substituição $\theta$. Seja $\mathcal{C}$ um conjunto de restrições de desigualdade.

- Se $\theta=\left\{A / a_{1}\right\}$ e $\mathcal{C}=\left\{A \neq a_{1}\right\}, \theta$ não é consistente com $\mathcal{C}$, pois $a_{1} \neq a_{1}$ é uma desigualdade inválida;

- Se $\theta=\left\{A / a_{1}, B / a_{1}\right\}$ e $\mathcal{C}=\{A \neq B\}, \theta$ não é consistente com $\mathcal{C}$, pois $\mathcal{C}[\theta]=\left\{a_{1} \neq\right.$ $\left.a_{1}\right\}$ contém desigualdade inválida;

- Se $\theta=\{B / A\}$ e $\mathcal{C}=\{A \neq B\}, \theta$ não é consistente com $\mathcal{C}$, pois $A \neq A$ é uma desigualdade inválida;

- Se $\theta=\left\{A / a_{1}, B / D\right\}$ e $\mathcal{C}=\left\{A \neq a_{2}, C \neq D, D \neq a_{1}\right\}, \theta$ é consistente com $\mathcal{C}$, pois $\mathcal{C}[\theta]=\left\{A \neq a_{2}, C \neq D, D \neq a_{1}\right\}$ não contém desigualdades inválidas;

Se o MGU não for consistente com todas as restrições dos parfactors envolvidos, as variáveis aleatórias parametrizadas representam conjuntos disjuntos de variáveis aleatórias. Se o MGU for consistente com todas as restrições dos parfactors envolvidos, as variáveis aleatórias parametrizadas representam conjuntos não disjuntos e possivelmente não idênticos de variáveis aleatórias. Neste caso, é necessário dividir as variáveis aleatórias parametrizadas de maneira a particionar o conjunto de variáveis aleatórias por elas representadas. 


\subsubsection{Particionamento de conjuntos}

Quando existe um MGU não vazio entre duas variáveis aleatórias parametrizadas que seja consistente com as restrições dos parfactors aos quais estas variáveis pertencem, é necessário realizar uma série de divisões de maneira a tornar os conjuntos de variáveis aleatórias iguais, conforme descrito por Poole (2003). O Algoritmo 7 ilustra o procedimento de divisão a partir do MGU e foi extraído de Kisynski (2010).

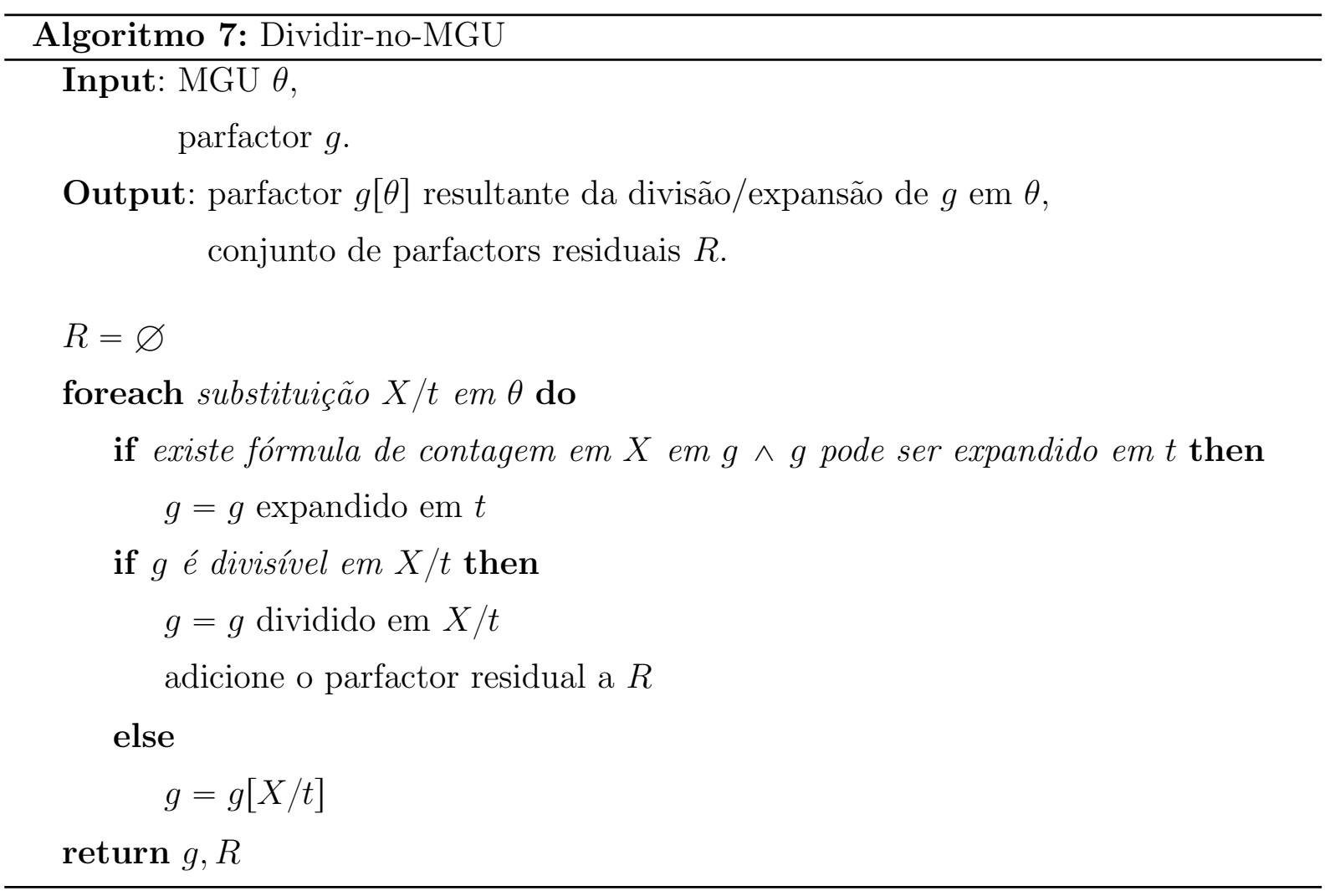

Exemplo 40. Considere os parfactors $g_{1}=\left\langle\varnothing,\left\{f(X), h\left(x_{1}\right)\right\}, \mathcal{F}_{1}\right\rangle$ e $g_{2}=\left\langle\varnothing,\left\{f\left(x_{2}\right), h\left(x_{2}\right)\right\}\right.$, $\left.\mathcal{F}_{2}\right\rangle$, cujo $M G U$ para a variável aleatória parametrizada $f$ é $\theta=\left\{X / x_{2}\right\}$. A divisão de $g_{1}$ em $\theta$ resulta em dois parfactors:

$$
\begin{aligned}
g_{1}[\theta] & =\left\langle\varnothing,\left\{f\left(x_{2}\right), h\left(x_{1}\right)\right\}, \mathcal{F}_{1}\right\rangle \\
g_{1}^{\prime} & =\left\langle\left\{X \neq x_{2}\right\},\left\{f(X), h\left(x_{1}\right)\right\}, \mathcal{F}_{1}\right\rangle
\end{aligned}
$$

$A$ divisão de $g_{2}$ em $\theta$ não altera o parfactor, pois $X \notin \operatorname{LV}\left(g_{2}\right)$.

Exemplo 41. Considere os parfactors $g=\left\langle\varnothing,\left\{p\left(A, x_{1}\right)\right\}, \mathcal{F}_{1}\right\rangle$ e $g_{A}=\langle\varnothing, p(A, B), c(B)$, $\varnothing, \mathbb{1}, \mathrm{OR}, A\rangle$, cujo $M G U$ para a variável aleatória parametrizada p é $\theta=\left\{B / x_{1}\right\}$. A divisão 
de $g_{A}$ em $\theta$ resulta em dois parfactors:

$$
\begin{aligned}
g_{A}[\theta] & =\left\langle\varnothing, p\left(A, x_{1}\right), c\left(x_{1}\right), \varnothing, \mathbb{1}, \mathrm{oR}, A\right\rangle \\
g_{A}^{\prime} & =\langle\varnothing, p(A, B), c(B), \varnothing, \mathbb{1}, \mathrm{OR}, A\rangle
\end{aligned}
$$

$A$ divisão de $g$ em $\theta$ não altera o parfactor, pois $B \notin \mathrm{LV}(g)$.

Após dividir os parfactors $g_{1}$ e $g_{2}$ no MGU, são obtidos dois conjuntos de parfactors residuais $R_{1}$ e $R_{2}$ e dois parfactors resultantes $g_{1}[\theta]$ e $g_{2}[\theta]$. A divisão no MGU faz com que as variáveis $\mathrm{f}\left(t_{1}^{1}, \ldots, t_{k}^{1}\right) \in g_{1}$ e $\mathrm{f}\left(t_{1}^{2}, \ldots, t_{k}^{2}\right) \in g_{2}$ sejam substituídas pela mesma variável aleatória parametrizada $\mathrm{f}\left(t_{1}, \ldots, t_{k}\right)$. Resta, no entanto, submeter esta variável às restrições de $g_{1}[\theta]$ e $g_{2}[\theta]$ e realizar as manipulações necessárias para que a variável aleatória parametrizada $\mathrm{f}\left(t_{1}, \ldots, t_{k}\right)$ represente o mesmo conjunto de variáveis aleatórias em $g_{1}[\theta]$ e $g_{2}[\theta]$. Este objetivo é alcançado através da divisão de cada parfactor nas restrições do outro. O Algoritmo 8 ilustra este procedimento e foi extraído e adaptado de Kisynski (2010).

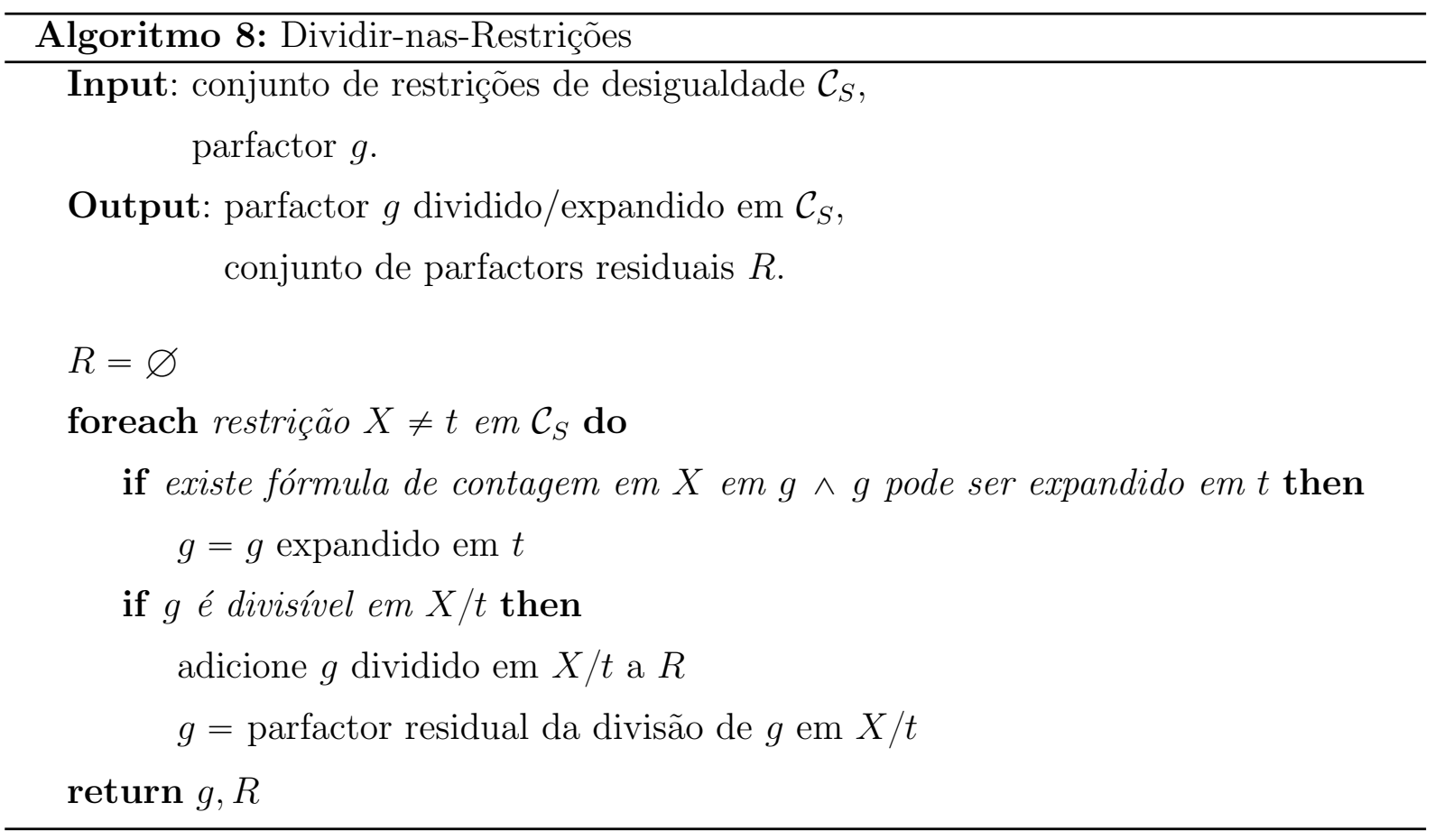

Dados dois parfactors $g_{1}^{\prime}$ e $g_{2}^{\prime}$ previamente unificados em uma variável aleatória parametrizada $f$, aplica-se o Algoritmo 8 nos pares $\left(g_{1}^{\prime}, \mathcal{C}_{2}^{\prime}\right)$ e $\left(g_{2}^{\prime}, \mathcal{C}_{1}^{\prime}\right)$. Ao final do procedimento, obtém-se dois parfactors $g_{1}^{\prime \prime}$ e $g_{2}^{\prime \prime} \operatorname{com}\left(\operatorname{RV}(f): \mathcal{C}_{1}^{\prime \prime}\right)=\left(\operatorname{RV}(f): \mathcal{C}_{2}^{\prime \prime}\right)$ e um conjunto de parfactors residuais. 
Exemplo 42. Considere os parfactors abaixo:

$$
\begin{array}{r}
\left\langle\left\{X \neq x_{1}\right\},\{f(X)\}, \mathcal{F}_{1}\right\rangle \\
\left\langle\left\{X \neq x_{2}, X \neq x_{3}\right\},\{f(X)\}, \mathcal{F}_{2}\right\rangle
\end{array}
$$

Suponha que $|\mathcal{D}(X)|>3$. Note que a variável aleatória parametrizada $f$ não representa o mesmo conjunto de variáveis aleatórias em $g_{1}$ e $g_{2}$. Para tornar estes conjuntos iguais, realiza-se uma divisão nas restrições. A divisão de $g_{1}$ nas restrições de $g_{2}$ resulta em três parfactors:

$$
\begin{array}{r}
\left\langle\varnothing,\left\{f\left(x_{2}\right)\right\}, \mathcal{F}_{1}\right\rangle \\
\left\langle\varnothing,\left\{f\left(x_{3}\right)\right\}, \mathcal{F}_{1}\right\rangle \\
\left\langle\left\{X \neq x_{1}, X \neq x_{2}, X \neq x_{3}\right\},\{f(X)\}, \mathcal{F}_{1}\right\rangle
\end{array}
$$

Por sua vez, a divisão de $g_{2}$ nas restrições de $g_{1}$ resulta em dois parfactors:

$$
\begin{array}{r}
\left\langle\varnothing,\left\{f\left(x_{1}\right)\right\}, \mathcal{F}_{2}\right\rangle \\
\left\langle\left\{X \neq x_{1}, X \neq x_{2}, X \neq x_{3}\right\},\{f(X)\}, \mathcal{F}_{2}\right\rangle
\end{array}
$$

$O$ conjunto $G=\left\{g_{1}, g_{2}\right\}$, após a divisão nas restrições, resulta no conjunto $G^{\prime}=\left\{g_{3}, g_{4}, g_{5}\right.$, $\left.g_{6}, g_{7}\right\}$. Tem-se ainda $\operatorname{RV}(f): \mathcal{C}_{5}=\operatorname{RV}(f): \mathcal{C}_{7}$ e os demais conjuntos de variáveis aleatórias representadas por $f$ são disjuntos entre si.

\subsubsection{Algoritmo de unificação completo}

O algoritmo de unificação completo é ilustrado no Algoritmo 9. 


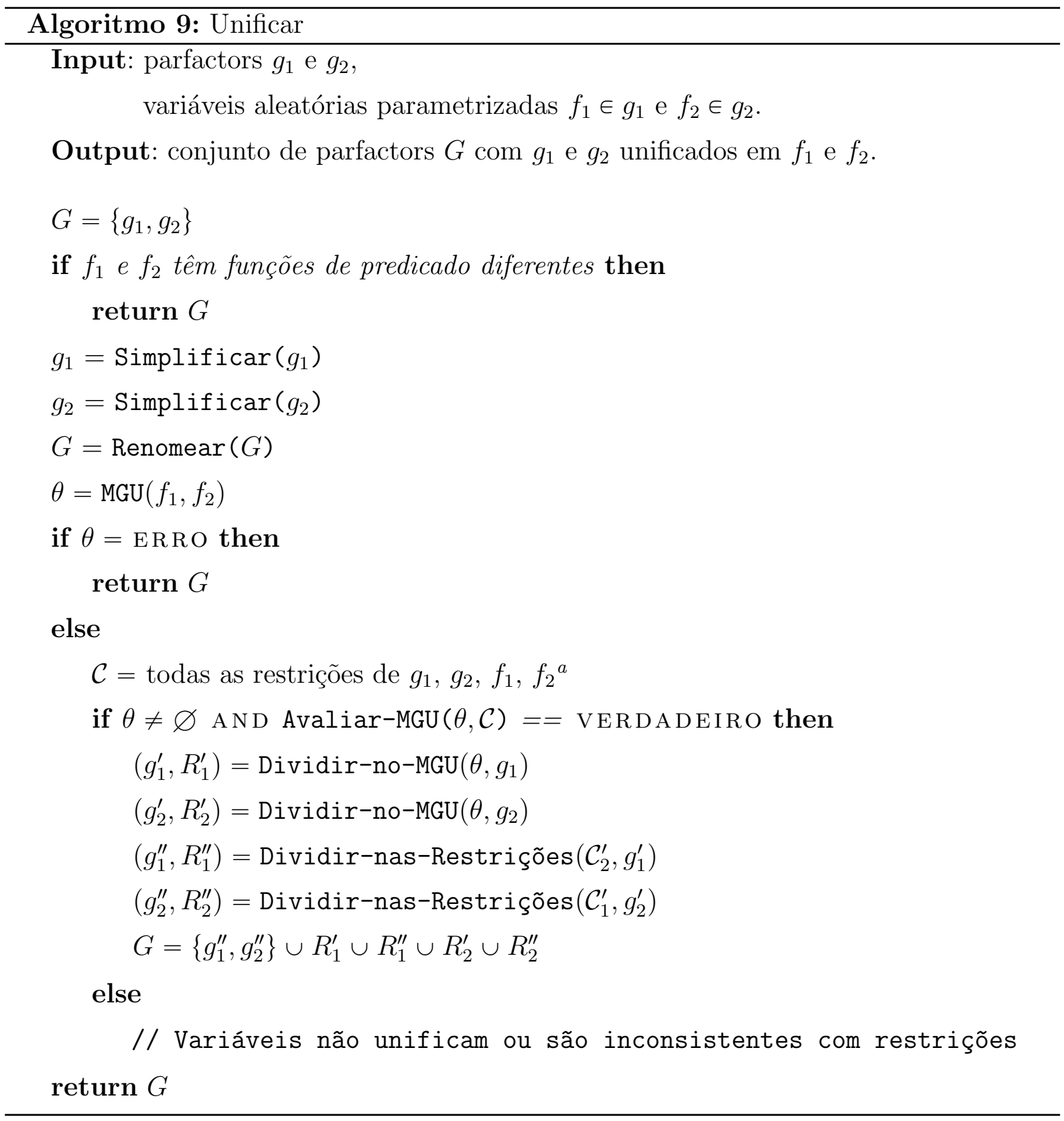

${ }^{a}$ Para os casos em que $f_{i}$ é uma fórmula de contagem.

Note que este procedimento pode gerar novas variáveis aleatórias parametrizadas quando houver parfactors de agregação devido ao resultado da divisão neste tipo de parfactor. Neste caso, as novas variáveis devem ser armazenadas para posterior eliminação pelo algoritmo.

Para manter a consistência de nomes das variáveis lógicas, deve-se renomeá-las para o antigo nome após a unificação. 
Exemplo 43. Considere o seguinte conjunto de parfactors:

$$
\begin{aligned}
G_{1}= & \left\langle\left\langle\left\{B \neq x_{2}, A \neq x_{4}\right\}, p(A, B), c(B),\{f(Y)\}, \mathbb{1}, \text { o R }, A\right\rangle\right. \\
& \left.\left\langle\left\{E \neq x_{3}\right\},\left\{p\left(x_{1}, E\right)\right\}, \mathcal{F}_{1}\right\rangle\right\}
\end{aligned}
$$

Suponha que $\mathcal{D}(A)=\mathcal{D}(B)=\mathcal{D}(E)=\left\{x_{1}, \ldots, x_{10}\right\}$ e $\mathcal{D}(Y)=\left\{y_{1}\right\}$. Ao final de cada passo do processo de unificação mostra-se o conjunto de parfactors resultante. Suponha que se deseja unificar a variável aleatória parametrizada $p$.

Inicialmente, as operações de pré-processamento são realizadas. Note que a variável lógica $Y$ está restrita a um indivíduo da população e portanto pode ser substituída. Não há necessidade de renomear as variáveis lógicas pois não há nomes ambíguos nos parfactors. Após o pré-processamento do conjunto $G_{1}$ obtém-se

$$
\begin{aligned}
G_{2}=\{ & \left\langle\left\{E \neq x_{3}\right\},\left\{p\left(x_{1}, E\right)\right\}, \mathcal{F}_{1}\right\rangle \\
& \left.\left\langle\left\{B \neq x_{2}, A \neq x_{4}\right\}, p(A, B), c(B),\left\{f\left(y_{1}\right)\right\}, \mathbb{1}, \mathrm{OR}, A\right\rangle\right\}
\end{aligned}
$$

Em seguida, obtém-se o $M G U$ para as variáveis $p(A, B)$ e $p\left(x_{1}, E\right)$. Ao aplicar o Algoritmo 5, o resultado retornado será $\theta=\left\{A / x_{1}, B / E\right\}$, que por sua vez é verificado com as restrições $\left\{A \neq x_{4}, B \neq x_{2}, E \neq x_{3}\right\}$ usando o Algoritmo 6. Como o MGU é consistente com todas as restrições, o próximo passo consiste em dividir $g_{2}$ e $g_{3}$ no $M G U \theta$. A divisão de $g_{3}$ na substituição $B / E$ resulta em

$$
\left\langle\left\{E \neq x_{2}, A \neq x_{4}\right\}, p(A, E), c(E),\left\{f\left(y_{1}\right)\right\}, \mathbb{1}, \mathrm{OR}, A\right\rangle
$$

Este parfactor é dividido na próxima substituição de $\theta, A / x_{1}$, resultando em

$$
\begin{aligned}
\sum_{\mathrm{Rv}\left(c^{\prime}\right)} \mathcal{J} & \left(\left\langle\{E \neq x_{2}, \overbrace{x_{1} \neq x_{4}}^{\text {sempre verdade }}\},\left\{p\left(x_{1}, E\right), f\left(y_{1}\right), c^{\prime}(E), c(E)\right\}, \mathcal{F}_{c}\right\rangle\right. \\
& \left.\left\langle\left\{E \neq x_{2}, A \neq x_{1}, A \neq x_{4}\right\}, p(A, E), c^{\prime}(E),\left\{f\left(y_{1}\right)\right\}, \mathbb{1}, \mathrm{OR}, A\right\rangle\right)
\end{aligned}
$$

em que $\mathcal{F}_{c}$ é calculado conforme a Proposição 10.

O parfactor $g_{2}$ passa pelo mesmo tratamento de $g_{3}$. Entretanto, como nem $A$ nem $B$ são variáveis lógicas que ocorrem em $g_{2}$, a divisão de $g_{2}$ no $M G U$ não altera o parfactor. 
Assim, ao final da etapa de divisão no MGU tem-se

$$
\begin{aligned}
\mathcal{J}\left(G_{2}\right)=\sum_{\mathrm{RV}\left(c^{\prime}\right)} \mathcal{J} & \left(\left\langle\left\{E \neq x_{3}\right\},\left\{p\left(x_{1}, E\right)\right\}, \mathcal{F}_{1}\right\rangle\right. \\
& \left\langle\left\{E \neq x_{2}\right\},\left\{p\left(x_{1}, E\right), f\left(y_{1}\right), c^{\prime}(E), c(E)\right\}, \mathcal{F}_{c}\right\rangle \\
& \left.\left\langle\left\{E \neq x_{2}, A \neq x_{1}, A \neq x_{4}\right\}, p(A, E), c^{\prime}(E),\left\{f\left(y_{1}\right)\right\}, \mathbb{1}, \mathrm{OR}, A\right\rangle\right)
\end{aligned}
$$

Note que a variável $p\left(x_{1}, E\right)$ aparece em $g_{2}$ e em $g_{4}$, porém não representa o mesmo conjunto de variáveis aleatórias nestes parfactors. Para torná-los iguais, é preciso realizar uma divisão nas restrições. A divisão de $g_{2}$ nas restrições de $g_{4}$ resulta nos parfactors

$$
\begin{aligned}
& \left\langle\{\overbrace{x_{2} \neq x_{3}}^{\text {sempre verdade }}\},\left\{p\left(x_{1}, x_{2}\right)\right\}, \mathcal{F}_{1}\right\rangle \\
& \left\langle\left\{E \neq x_{2}, E \neq x_{3}\right\},\left\{p\left(x_{1}, E\right)\right\}, \mathcal{F}_{1}\right\rangle
\end{aligned}
$$

A divisão de $g_{4}$ nas restrições de $g_{2}$ resulta em

$$
\begin{aligned}
& \left\langle\{\overbrace{x_{2} \neq x_{3}}^{\text {sempre verdade }}\},\left\{p\left(x_{1}, x_{3}\right), f\left(y_{1}\right), c^{\prime}\left(x_{3}\right), c\left(x_{3}\right)\right\}, \mathcal{F}_{c}\right\rangle \\
& \left\langle\left\{E \neq x_{2}, E \neq x_{3}\right\},\left\{p\left(x_{1}, E\right), f\left(y_{1}\right), c^{\prime}(E), c(E)\right\}, \mathcal{F}_{c}\right\rangle
\end{aligned}
$$

Após a divisão nas restrições o conjunto de parfactors se torna:

$$
\begin{aligned}
& \mathcal{J}\left(G_{2}\right)=\sum_{\mathrm{RV}\left(c^{\prime}\right)} \mathcal{J}\left(\left\langle\left\{E \neq x_{2}, A \neq x_{1}, A \neq x_{4}\right\}, p(A, E), c^{\prime}(E),\left\{f\left(y_{1}\right)\right\}, \mathbb{1}, \mathrm{OR}, A\right\rangle, \quad\left(g_{5}\right)\right. \\
& \left\langle\varnothing,\left\{p\left(x_{1}, x_{2}\right)\right\}, \mathcal{F}_{1}\right\rangle, \\
& \left\langle\left\{E \neq x_{2}, E \neq x_{3}\right\},\left\{p\left(x_{1}, E\right)\right\}, \mathcal{F}_{1}\right\rangle \text {, } \\
& \left\langle\varnothing,\left\{p\left(x_{1}, x_{3}\right), f\left(y_{1}\right), c^{\prime}\left(x_{3}\right), c\left(x_{3}\right)\right\}, \mathcal{F}_{c}\right\rangle, \\
& \left.\left\langle\left\{E \neq x_{2}, E \neq x_{3}\right\},\left\{p\left(x_{1}, E\right), f\left(y_{1}\right), c^{\prime}(E), c(E)\right\}, \mathcal{F}_{c}\right\rangle\right)
\end{aligned}
$$

Note que agora os conjuntos de variáveis aleatórias representados por $p\left(x_{1}, E\right)$ em $g_{7}$ e $g_{9}$ são iguais. Para cada par de parfactors resultantes, os conjuntos de variáveis aleatórias representadas por $p$ são sempre disjuntos ou iguais. Assim, os parfactors deste conjunto estão prontos para serem multiplicados ou sofrerem eliminação.

\subsection{Macro-operações}

Como visto na Seção 3.2, as operações de Divisão, Proposicionalização, Expansão, Conversão e Contagem manipulam um conjunto de parfactors de maneira a permitir a 
Multiplicação em primeira ordem, o que por sua vez é utilizada para habilitar a operação de Eliminação. Para que o algoritmo encontre melhores ordenações de operações, o A C- F OVE combina as operações elementares em macro-operações (MILCH et al., 2008).

O A C-F OVE é um algoritmo de busca gulosa. Enquanto houverem variáveis aleatórias para eliminar, o AC-FOVE enumera todas as possíveis macro-operações e escolhe aquela com menor custo. O custo de uma macro-operação é baseado em dois critérios:

- O número de variáveis aleatórias que a macro-operação elimina. Quanto maior este número, menor é o custo;

- O tamanho do fator que a macro-operação gera. Quanto maior este número, maior é o custo.

O primeiro critério tem precedência sobre o segundo, isto é, quando há empate no número de variáveis aleatórias eliminadas, o tamanho do fator gerado é usado como critério de desempate. Caso haja empate também no segundo critério, o algoritmo sorteia uma operação (KISYNSKI, 2010). Embora o custo das macro-operações do algoritmo A C- F OVE tenha sido definido como "o tamanho do fator que a operação gera" (KISYNSKI; POOLE, 2009), não foram fornecidas fórmulas analíticas que permitissem o seu cálculo. Neste trabalho estas fórmulas serão formalizadas nas Proposições 14, 15, 16, 17 e 18, juntamente com as respectivas demonstrações propostas pelo autor deste trabalho.

As macro-operações são apresentadas a seguir e, para cada uma delas, propõe-se uma fórmula para calcular seu custo.

\subsubsection{Particionamento}

O Particionamento consiste em manipular um conjunto de parfactors de tal maneira que, dadas duas variáveis aleatórias parametrizadas de parfactors distintos, os conjuntos de variáveis aleatórias por elas representados sejam iguais ou disjuntos. Esta macro-operação aplica a operação de unificação para todos os possíveis pares de variáveis aleatórias parametrizadas pertencentes a parfactors distintos. O Algoritmo 10 ilustra esta macrooperação. 


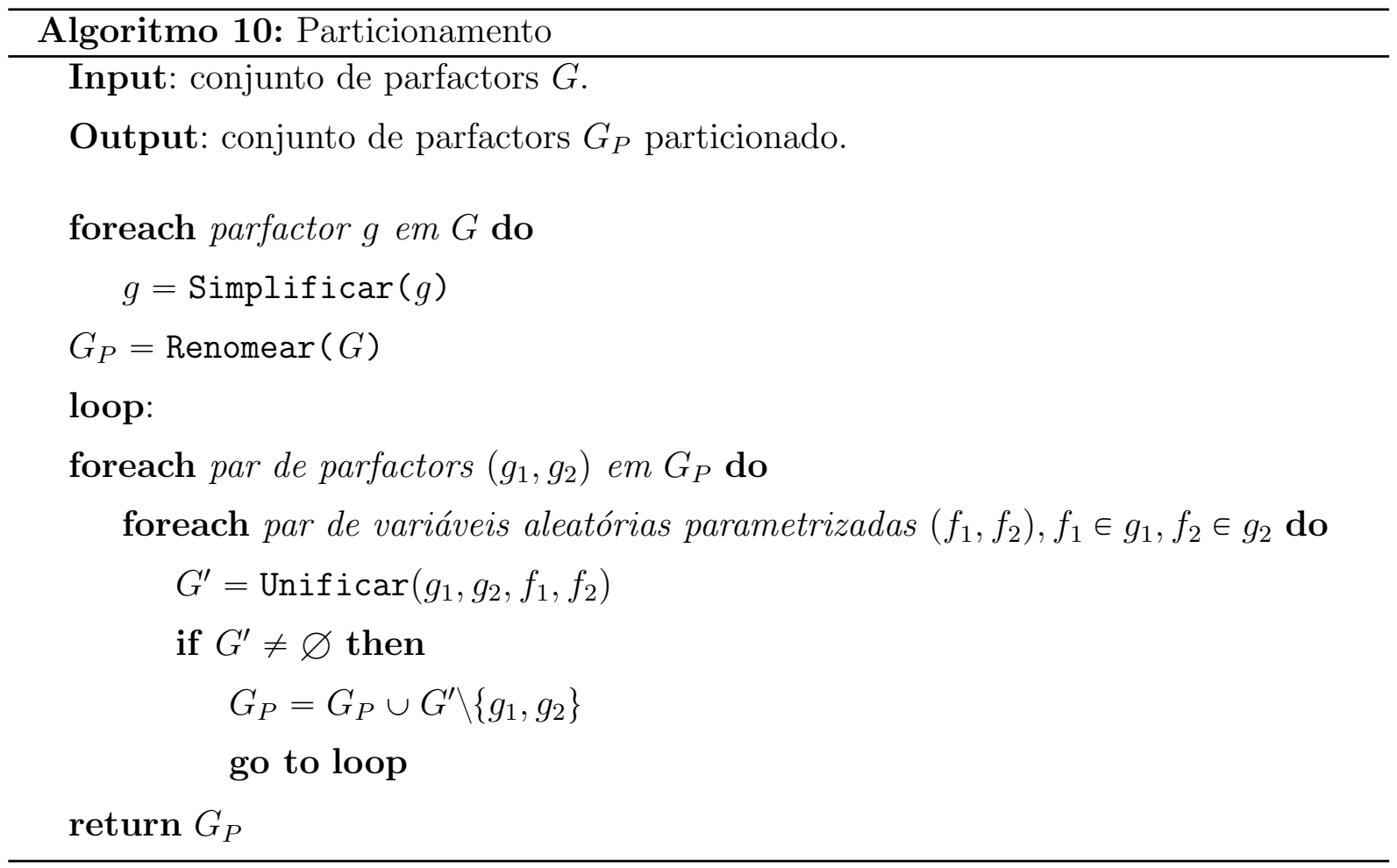

O Particionamento tem a particularidade de não ser utilizado na seleção de macrooperações pelo AC-FOVE. Ao invés disso, ele é usado como auxiliar de outras macrooperações. Após o particionamento de um conjunto de parfactors, seus elementos podem ser multiplicados para permitir uma posterior eliminação. Por não entrar na avaliação de possíveis operações a serem executadas pelo A C-F OVE, esta macro-operação não possui um custo associado.

\subsubsection{Eliminação global}

Dado um conjunto de parfactors $G$, uma variável aleatória parametrizada $f$ e um conjunto de restrições $\mathcal{C}$, a Eliminação Global consiste em eliminar o conjunto de variáveis aleatórias $\operatorname{RV}(f): \mathcal{C}$ dos parfactors em $G$. Isto é feito multiplicando-se todos os parfactors de $G$ que envolvem o conjunto $\operatorname{Rv}(f): \mathcal{C}$ e eliminando a variável $f$ do produto.

A multiplicação sempre é possível após um Particionamento, mas nem sempre é possível eliminar uma variável aleatória parametrizada do produto. Quando isto ocorre, a macrooperação restaura o conjunto $G$ ao estado anterior à multiplicação. Uma outra alternativa seria preservar o produto e realizar a contagem das variáveis lógicas que impedem a eliminação, mas esta opção não será contemplada neste trabalho, pois a contagem é uma operação relativamente cara (vide Proposição 15) que torna o tamanho dos fatores envolvidos dependente do tamanho da população das variáveis lógicas contadas. O Algoritmo 
11 ilustra a Eliminação Global.

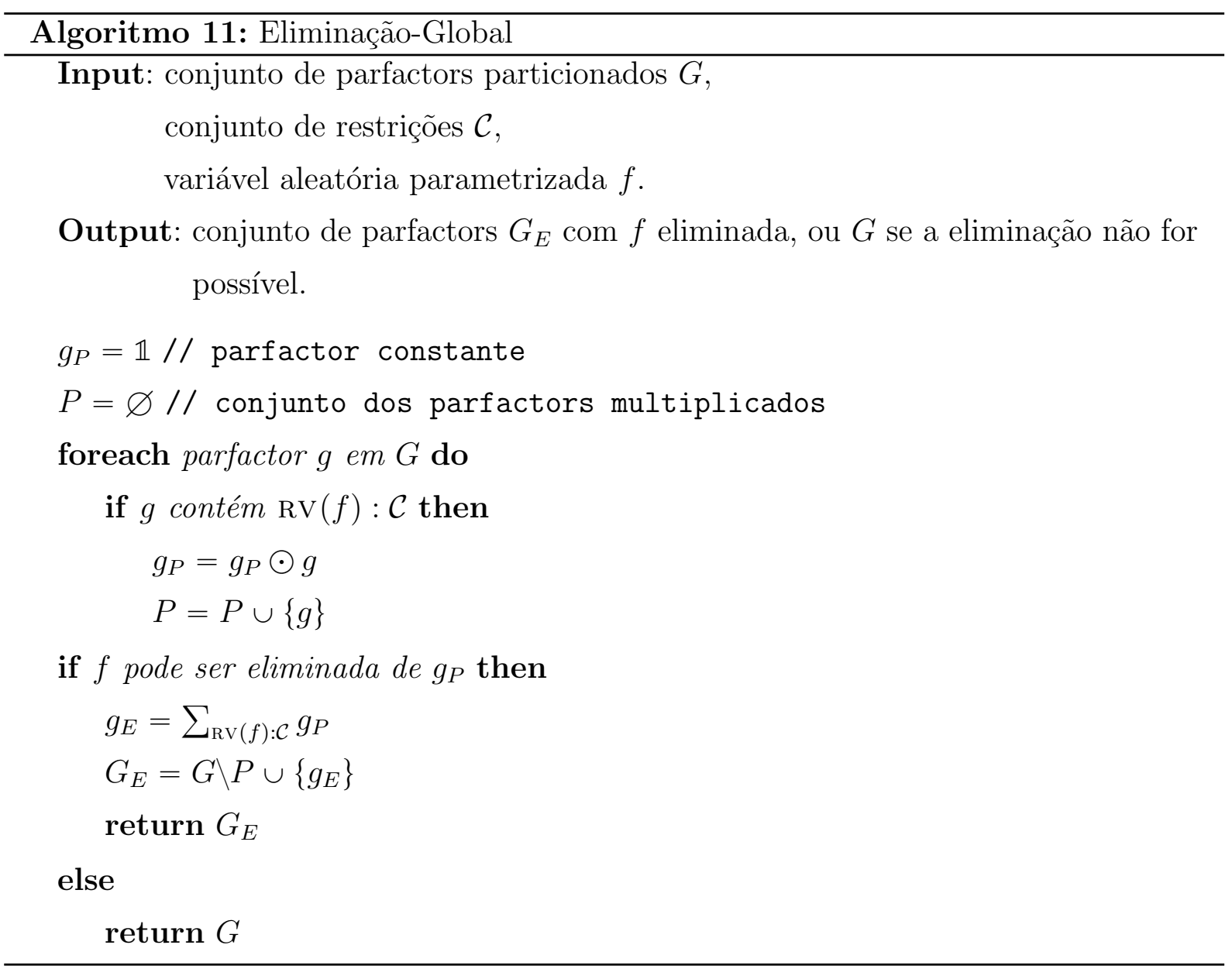

A Eliminação Global é a única macro-operação que elimina variáveis aleatórias. O número de variáveis aleatórias eliminadas é $|\operatorname{RV}(f): \mathcal{C}|=\prod_{X \in \operatorname{PARAM}(f)}|\mathcal{D}(X): \mathcal{C}|$. Havendo empate no critério de variáveis eliminadas, calcula-se o tamanho do fator resultante.

A Proposição 14 estabelece o custo da Eliminação Global. O Lema 1 será usado como auxiliar para deduzir o custo das macro-operações.

Lema 1. Seja $\mathcal{F}$ um fator nas variáveis aleatórias parametrizadas do conjunto $\mathcal{V}=\left\{f_{1}\right.$, $\left.\ldots, f_{n}\right\}$. O tamanho do fator $\mathcal{F}$, denotado por $|\mathcal{F}|$, é dado por

$$
|\mathcal{F}|=\prod_{v \in \mathcal{V}}|\operatorname{RANGE}(v)|
$$

Demonstração. O fator $\mathcal{F}$ é uma função $f: \operatorname{RANGE}\left(f_{1}\right) \times \cdots \times \operatorname{RANGE}\left(f_{n}\right) \rightarrow \mathbb{R}$. O tamanho do domínio de $f$ é igual ao tamanho de $\mathcal{F}$ e é obtido pela combinação de todos os possíveis elementos dos domínios das variáveis $f_{1}, \ldots, f_{n}$, que é $\left|\operatorname{RANGE}\left(f_{1}\right)\right| \cdots \ldots$ $\left|\operatorname{RANGE}\left(f_{n}\right)\right|=\prod_{v \in \mathcal{V}}|\operatorname{RANGE}(v)|$.

Proposição 14. Considere um conjunto de parfactors $G$. Seja $P$ o subconjunto de par- 
factors de $G$ que contém as variáveis aleatórias de $\mathrm{RV}(f): \mathcal{C}$ e seja $\mathcal{V}_{P}$ o conjunto das variáveis aleatórias parametrizadas do produto dos parfactors de P. O tamanho do fator resultante da Eliminação Global é

$$
\operatorname{Custo}(\operatorname{EliminaÇ} \tilde{A} O-\operatorname{GlobaL}(G, \mathcal{C}, f))=\prod_{v \in \mathcal{V}_{P} \backslash\{f\}}|\operatorname{RAnGE}(v)|
$$

Demonstração. A Eliminação Global é dividida em duas fases: na primeira, multiplicam-se os parfactors do conjunto $P$. Na segunda, eliminam-se as variáveis aleatórias $\operatorname{RV}(f): \mathcal{C}$ do produto obtido. Após esta eliminação, o parfactor resultante possuirá as variáveis aleatórias parametrizadas do conjunto $\mathcal{V}_{P} \backslash\{f\}$. Pelo Lema 1 , o fator resultante terá tamanho $\prod_{v \in \mathcal{V}_{P} \backslash\{f\}}|\operatorname{RANGE}(v)|$.

\subsubsection{Contagem}

A Contagem consiste em eliminar uma variável lógica de um parfactor. Esta operação pode ser usada para satisfazer a Condição 2 da Proposição 2. A Contagem não se aplica a parfactors de agregação, visto que já existe uma variável aleatória parametrizada implicitamente "contada" no parfactor.

A contagem é simplesmente a aplicação da Proposição 13. O Algoritmo 12 ilustra a macro-operação.

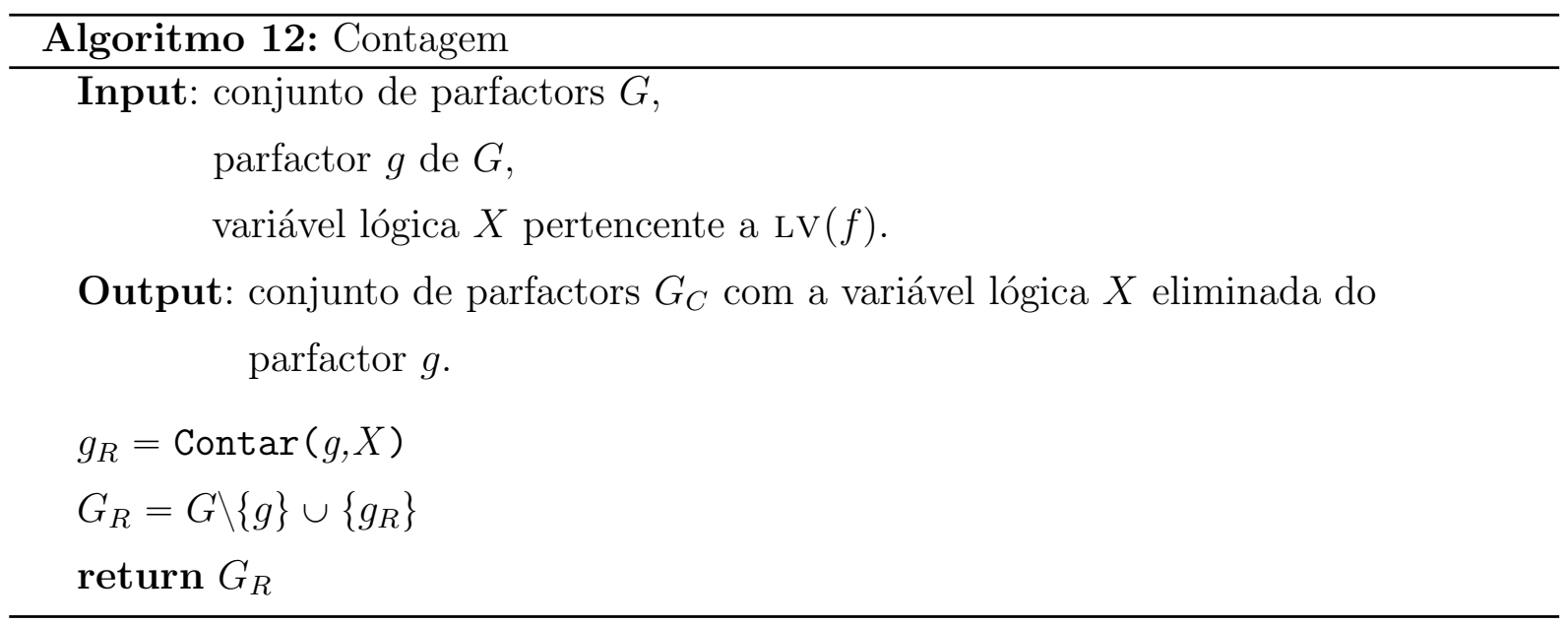

O custo da da macro-operação de Contagem é definido na Proposição 15.

Proposição 15. Seja $g$ um parfactor cujo fator é $\mathcal{F}$ e $X$ uma variável lógica de g. Seja $\mathcal{F}_{c}$ o fator gerado pela contagem de $X$ em $\mathrm{g}$. O tamanho do fator $\mathcal{F}_{c}$ é dado por:

$$
\operatorname{Custo}(\operatorname{Contagem}(G, g, X))=\frac{\left(\begin{array}{c}
|\mathcal{D}(X): \mathcal{C}|+|\operatorname{Range}(f)|-1 \\
|\operatorname{Range}(f)|-1
\end{array}\right)}{|\operatorname{Range}(f)|} \times|\mathcal{F}|
$$


Demonstração. Seja $\mathcal{V}$ o conjunto das variáveis aleatórias parametrizadas de $g$. Após a contagem de $X$, o parfactor resultante $g_{c}$ terá um conjunto de variáveis aleatórias parametrizadas dado por $\mathcal{V} \backslash\{f\} \cup\left\{\#_{X: \mathcal{C}_{X}}[f]\right\}$ (vide Proposição 13). Pelo Lema 1, o tamanho do fator $\mathcal{F}_{c}$ de $g_{c}$ é dado por

$$
\begin{aligned}
\left|\mathcal{F}_{c}\right| & =\prod_{v \in \mathcal{V} \backslash\{f\} \cup\left\{\# X: \mathcal{C}_{X}[f]\right\}}|\operatorname{RANGE}(v)| \\
& =\frac{\left|\operatorname{RANGE}\left(\# \#_{X: C_{X}}[f]\right)\right|}{|\operatorname{RANGE}(f)|} \times \prod_{v \in \mathcal{V}}|\operatorname{RANGE}(v)| \\
& =\frac{\left(\begin{array}{c}
|\mathcal{D}(X): \mathcal{C}|+|\operatorname{RangE}(f)|-1 \\
|\operatorname{RANGE}(f)|-1
\end{array}\right)}{|\operatorname{RANGE}(f)|} \times|\mathcal{F}| .
\end{aligned}
$$

\subsubsection{Proposicionalização}

A Proposicionalização de uma variável lógica $X$ em um parfactor $g$ consiste em dividir $g$ para todo indivíduo da população atrelada a $X$ que satisfaz as restrições de $g$. Sempre é possível proposicionalizar uma variável lógica (KISYNSKI, 2010), mas esta operação deve ser evitada sempre que possível pois ela destrói o objetivo da inferência em primeira ordem. Entretanto, em algumas situações ela é a única operação possível. Se todas as variáveis lógicas de um conjunto de parfactors forem proposicionalizadas, o problema de inferência pode ser resolvido com os algoritmos de inferência existentes em redes Bayesianas (por exemplo, a Eliminação de Variáveis de Zhang e Poole (1994)). O Algoritmo 13 ilustra a Proposicionalização. 


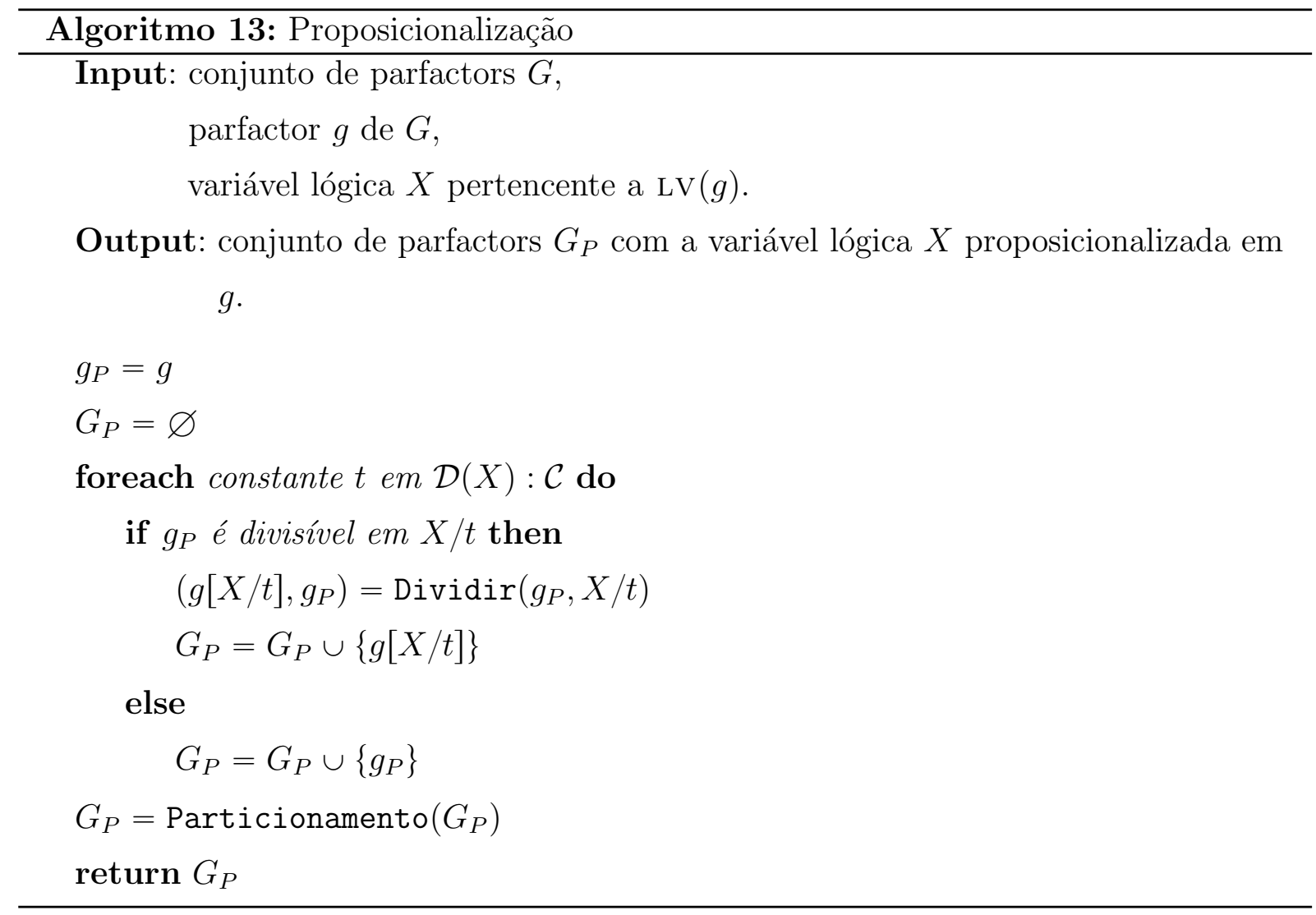

Após a Proposicionalização é feito um Particionamento para garantir que os conjuntos de variáveis aleatórias dos parfactors sejam iguais ou disjuntos entre si. O Particionamento gera $|\mathcal{D}(X): \mathcal{C}|$ novos parfactors, cada qual com um fator de tamanho $|\mathcal{F}|$. Para que esta operação seja escolhida apenas em último caso, convém tornar o custo dela alto. Para tanto, utiliza-se o tamanho do fator resultante da multiplicação de todos os parfactors gerados pela Proposicionalização. O custo desta macro-operação é detalhado na Proposição 16.

Proposição 16. Seja $\mathcal{V}$ o conjunto de variáveis aleatórias parametrizadas de g. Então

$$
\text { Custo(Proposicionaliza Ç } \tilde{A} \mathrm{O}(G, g, X))=\prod_{f \in \mathcal{V}}|\mathrm{R} \operatorname{Ang}(f)|^{\delta(f, X)}
$$

em que

$$
\delta(f, X)= \begin{cases}|\mathcal{D}(X): \mathcal{C}| & \text { se } X \in \text { PA R A M }(f) \\ 1 & \text { caso contrário. }\end{cases}
$$

Demonstração. Para esta demonstração, as variáveis aleatórias parametrizadas de $g$ serão divididas em dois grupos: aquelas que contêm a variável lógica $\mathrm{X}$ ficarão no conjunto $\mathcal{V}_{X}$, enquanto que as demais serão colocadas no conjunto $\mathcal{V}_{\bar{X}}$. Pelo Lema 1 , tem-se

$$
|\mathcal{F}|=\prod_{v \in \mathcal{V}}|\operatorname{RANGE}(v)|=\prod_{v \in \mathcal{V}_{X}}|\operatorname{RANGE}(v)| \times \prod_{v \in \mathcal{V}_{\bar{X}}}|\operatorname{RANGE}(v)| .
$$


Seja $\mathcal{D}(X): \mathcal{C}=\left\{x_{1}, \ldots, x_{n}\right\}$. Ao proposicionalizar $X$, o parfactor $g$ se transforma em $|\mathcal{D}(X): \mathcal{C}|$ novos parfactors: $g\left[X / x_{1}\right], \ldots, g\left[X / x_{n}\right]$. A multiplicação destes parfactors produz um conjunto de variáveis aleatórias parametrizadas $\mathcal{V}_{p}$ dado por

$$
\begin{aligned}
\mathcal{V}_{p} & =\left(\mathcal{V}_{X}\left[X / x_{1}\right] \cup \mathcal{V}_{\bar{X}}\left[X / x_{1}\right]\right) \cup \cdots \cup\left(\mathcal{V}_{X}\left[X / x_{n}\right] \cup \mathcal{V}_{\bar{X}}\left[X / x_{n}\right]\right) \\
& =\mathcal{V}_{X}\left[X / x_{1}\right] \cup \cdots \cup \mathcal{V}_{X}\left[X / x_{n}\right] \cup \mathcal{V}_{\bar{X}}
\end{aligned}
$$

pois $\mathcal{V}_{\bar{X}}\left[X / x_{i}\right]=\mathcal{V}_{\bar{X}}$ para qualquer $i=1, \ldots, n$. Então,

$$
\left|\mathcal{F}_{p}\right|=\prod_{v \in \mathcal{V}_{X}\left[X / x_{1}\right]}|\operatorname{RANGE}(v)| \times \cdots \times \prod_{v \in \mathcal{V}_{X}\left[X / x_{n}\right]}|\operatorname{RANGE}(v)| \times \prod_{v \in \mathcal{V}_{\bar{X}}}|\operatorname{RANGE}(v)| .
$$

Note que $\prod_{v \in \mathcal{V}_{X}\left[X / x_{i}\right]}|\operatorname{RANGE}(v)|=\prod_{v \in \mathcal{V}_{X}}|\operatorname{RANGE}(v)|$, pois o valor de RANGE $(v)$ não se altera com a proposicionalização. Logo,

$$
\begin{aligned}
\left|\mathcal{F}_{p}\right| & =\left(\prod_{v \in \mathcal{V}_{X}}|\operatorname{RANGE}(v)|\right)^{|\mathcal{D}(X): \mathcal{C}|} \times \prod_{v \in \mathcal{V}_{\bar{X}}}|\operatorname{RANGE}(v)| \\
& =\prod_{v \in \mathcal{V}_{X}}|\operatorname{RANGE}(v)|^{|\mathcal{D}(X): \mathcal{C}|} \times \prod_{v \in \mathcal{V}_{\bar{X}}}|\operatorname{RANGE}(v)|
\end{aligned}
$$

Defina a função $\delta(f, X)$ dada por

$$
\delta(f, X)= \begin{cases}|\mathcal{D}(X): \mathcal{C}| & \text { se } X \in \text { PA R A M }(f) \\ 1 & \text { caso contrário. }\end{cases}
$$

Então a Equação (3.3.6) pode ser reescrita como

$$
\left|\mathcal{F}_{p}\right|=\prod_{v \in \mathcal{V}}|\operatorname{RANGE}(v)|^{\delta(v, X)}
$$

\subsubsection{Expansão total}

A Expansão Total é uma macro-operação análoga à Proposicionalização quando a variável lógica está atrelada a uma fórmula de contagem. Esta operação consiste em expandir uma fórmula de contagem $\#_{X: \mathcal{C}_{X}}[f]$ para cada indivíduo de $\mathcal{D}(X): \mathcal{C}_{X}$. Sempre é possível expandir totalmente uma fórmula de contagem (KISYNSKI, 2010), mas assim como a Proposicionalização, deve ser evitada pois torna o tempo de inferência dependente do tamanho da população de $X$.

O Algoritmo 14 ilustra a Expansão Total. 


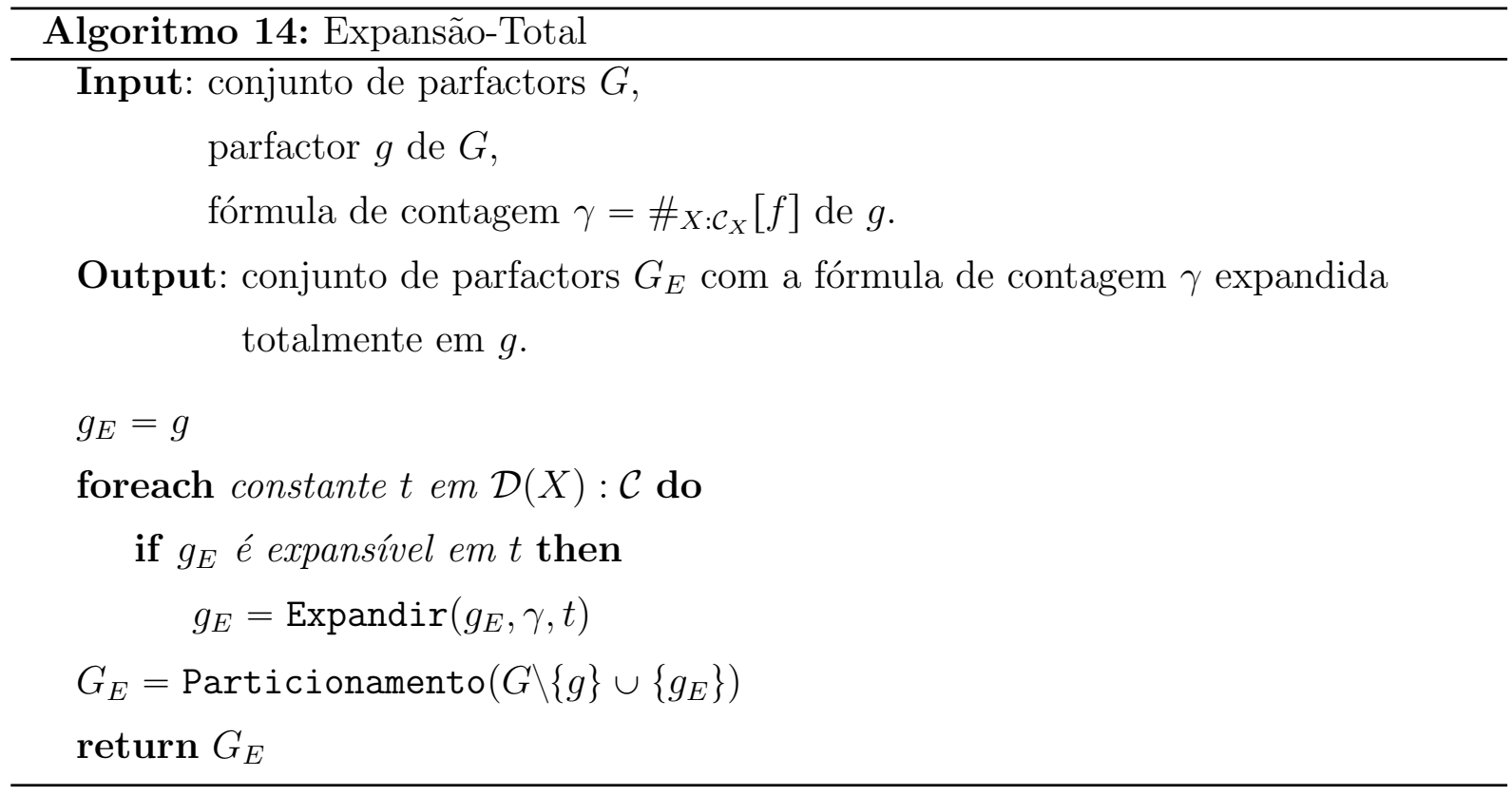

Após a Expansão Total é feito um Particionamento para garantir que os conjuntos de variáveis aleatórias dos parfactors sejam iguais ou disjuntos entre si. Assim como na Proposicionalização, é conveniente que o custo desta operação seja alto para que ela seja escolhida somente quando for a única alternativa viável, isto é, não é possível realizar Eliminação Global, Contagem ou Conversão. O tamanho do fator gerado por esta operação é explicitado na Proposição 17.

Proposição 17. Considere o parfactor g que contém uma fórmula de contagem $\#_{X: \mathcal{C}_{X}}[f]$. Seja $\mathcal{F}$ o fator contido em $g$. A expansão total da variável lógica $X$ no parfactor $g$ resulta em um parfactor cujo fator tem tamanho

$$
\operatorname{Custo}(\operatorname{Expans} \tilde{A} O-\operatorname{Total}(G, g, X))=\frac{\left.|\operatorname{RANGE}(f)|\right|^{\left|\mathcal{D}(X): \mathcal{C}_{X}\right|}}{\left.\mid \operatorname{RANGE}_{X: \mathcal{C}_{X}}[f]\right) \mid} \times|\mathcal{F}|
$$

Demonstração. Seja $\mathcal{V}=\left\{\#_{X: \mathcal{C}_{X}}[f]\right\} \cup \mathcal{V}^{\prime}$ o conjunto das variáveis aleatórias parametrizadas do parfactor $g$. Após a expansão total de $g$, a fórmula de contagem $\#_{X: \mathcal{C}_{X}}[f]$ é substituída por $f\left[X / x_{1}\right], \ldots, f\left[X / x_{n}\right]$ para $\mathcal{D}(X): \mathcal{C}_{X}=\left\{x_{1}, \ldots, x_{n}\right\}$. Então, o conjunto de variáveis aleatórias parametrizadas se torna $\mathcal{V}_{e}=\left\{f\left[X / x_{1}\right], \ldots, f\left[X / x_{n}\right]\right\} \cup \mathcal{V}^{\prime}$, e pelo 
Lema 1, tem-se

$$
\begin{aligned}
\left|\mathcal{F}_{e}\right| & =\prod_{v \in \mathcal{V}_{e}}|\operatorname{RANGE}(v)| \\
& =|\operatorname{RANGE}(f)|^{\left|\mathcal{D}(X): \mathcal{C}_{X}\right|} \times \prod_{v \in \mathcal{V}^{\prime}}|\operatorname{RANGE}(v)| \\
& =|\operatorname{RANGE}(f)|^{\left|\mathcal{D}(X): \mathcal{C}_{X}\right|} \times \frac{\prod_{v \in \mathcal{V}}|\operatorname{RANGE}(v)|}{\left|\operatorname{RANGE}\left(\# X: \mathcal{C}_{X}[f]\right)\right|} \\
& =\frac{|\operatorname{RANGE}(f)|^{\left|\mathcal{D}(X): \mathcal{C}_{X}\right|}}{\left|\operatorname{RANGE}\left(\# X: \mathcal{C}_{X}[f]\right)\right|} \times|\mathcal{F}|
\end{aligned}
$$

\subsubsection{Conversão}

A Conversão consiste me transformar um parfactor de agregação em parfactors na forma padrão. Esta macro-operação é uma aplicação direta da Proposição 12 e é ilustrada no Algoritmo 15.

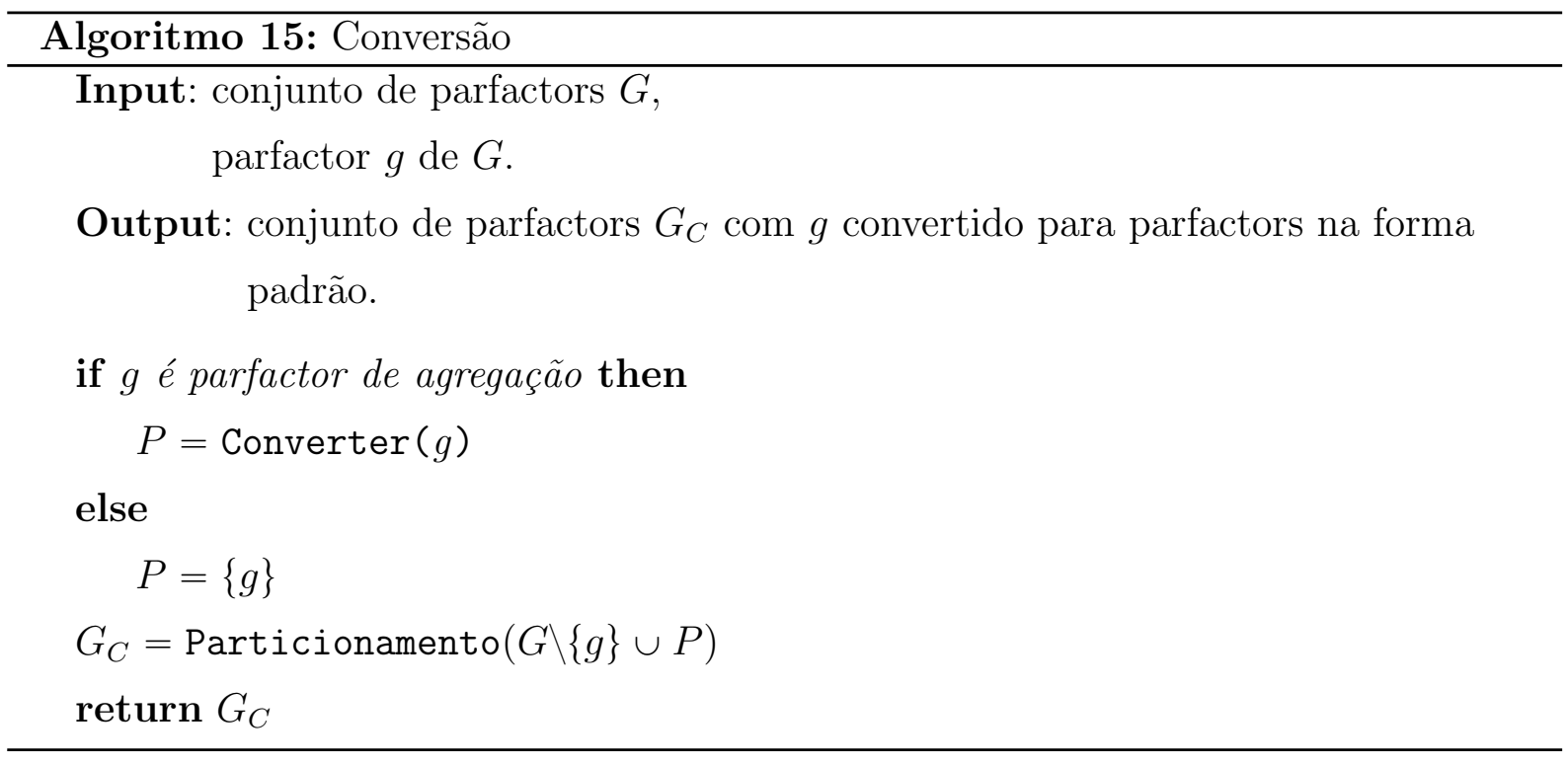

O custo desta operação é demonstrado na Proposição 18.

Proposição 18. Seja $g=\left\langle\mathcal{C}, p, c, V, \mathcal{F}_{p}, \otimes, A\right\rangle$ um parfactor de agregação. O custo da conversão de g para parfactor na forma padrão é dado por

$$
\operatorname{Custo}(\operatorname{Convers} \tilde{A} O(G, g))=\prod_{f \in\left\{\#_{A: \mathcal{C}_{A}}[p], c\right\} \cup \mathcal{V}}|\operatorname{RANGE}(f)|
$$

Demonstração. A conversão de um parfactor de agregação $g=\left\langle\mathcal{C}, p, c, V, \mathcal{F}_{p}, \otimes, A\right\rangle$ resulta, de acordo com a Proposição 12, em dois parfactors: $g_{1}=\left\langle\mathcal{C},\{p\} \cup V, \mathcal{F}_{p}\right\rangle$ e $g_{2}=\left\langle\mathcal{C} \backslash \mathcal{C}_{A}\right.$, 
$\left.\left\{\#_{A: \mathcal{C}_{A}}[p], c\right\} \cup V, \mathcal{F}_{\#}\right\rangle$. Como R A GE $(p) \subseteq \operatorname{RANGE}(c)$, o tamanho do fator $\mathcal{F}_{\#}$ é sempre maior que o tamanho do fator $\mathcal{F}_{p}$. O tamanho do fator $\mathcal{F}_{\#}$, pelo Lema 1 , será

$$
\left|\mathcal{F}_{\#}\right|=\prod_{f \in\left\{\# A_{A: \mathcal{C}_{A}}[p], c\right\} \cup \mathcal{V}}|\operatorname{RANGE}(f)|
$$

\section{$3.4 \mathrm{O}$ algoritmo}

Nesta seção será apresentado o algoritmo A C - F OVE com base nas macro-operações vistas na Seção 3.3. Dado um conjunto de parfactors $G$ e um conjunto de variáveis aleatórias Q, o algoritmo A C-F OVE realiza a eliminação em primeira ordem das variáveis que não estão em Q. Quando isto não é possível, as demais macro-operações (Conversão, Proposicionalização, Expansão Total e Contagem) são utilizadas para viabilizar a Eliminação Global.

Através das macro-operações de Proposicionalização e Expansão Total (e Conversão para parfactors de agregação), sempre é possível reduzir uma rede de primeira ordem a uma rede de ordem zero (KISYNSKI, 2010), portanto no pior caso o A C-F OVE se comporta como o algoritmo de Eliminação de Variáveis (ZHANG; POOLE, 1996).

O Algoritmo 16 ilustra o pseudocódigo para o AC-F OVE. Esta listagem pressupõe a existência de três variáveis globais:

op: estrutura de dados que armazena a macro-operação atual para execução. Utiliza-se o símbolo $\varnothing$ para indicar que não existe macro-operação atribuída a esta variável.

n: número de variáveis aleatórias eliminadas pela operação op. Será diferente de zero somente quando op é uma Eliminação Global.

s: custo da operação op. Utiliza-se o símbolo $\infty$ para indicar que a operação op é impossível. 


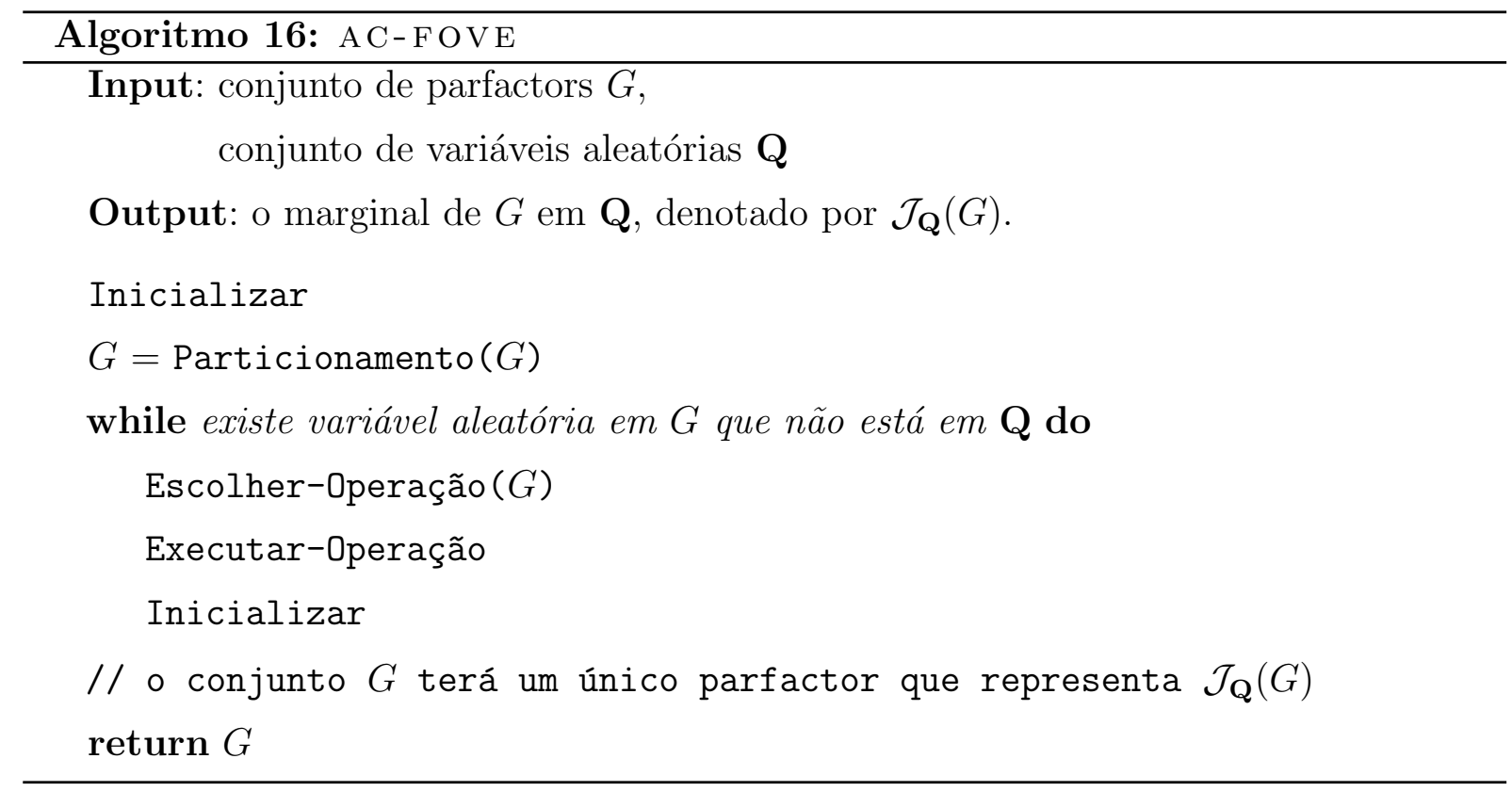

\section{Procedimento Inicializar}

$$
\begin{aligned}
& \mathrm{op}=\varnothing \\
& \mathrm{n}=0 \\
& \mathrm{~s}=\infty
\end{aligned}
$$

Procedimento Escolher-Operacao $(G)$

Input: conjunto de parfactors $G$

foreach parfactor $g$ em $G$ do

foreach variável aleatória parametrizada $f$ em $g$ do

Avaliar (Expansão-Total $(G, g, f))$;

Avaliar(Eliminação-Global $(G, \mathcal{C} \in g, f)$ );

foreach variável lógica $X$ em $g$ do

Avaliar (Contagem $(G, g, X))$;

Avaliar (Proposicionalização $(G, g, X))$;

Avaliar (Conversão $(G, g))$; 

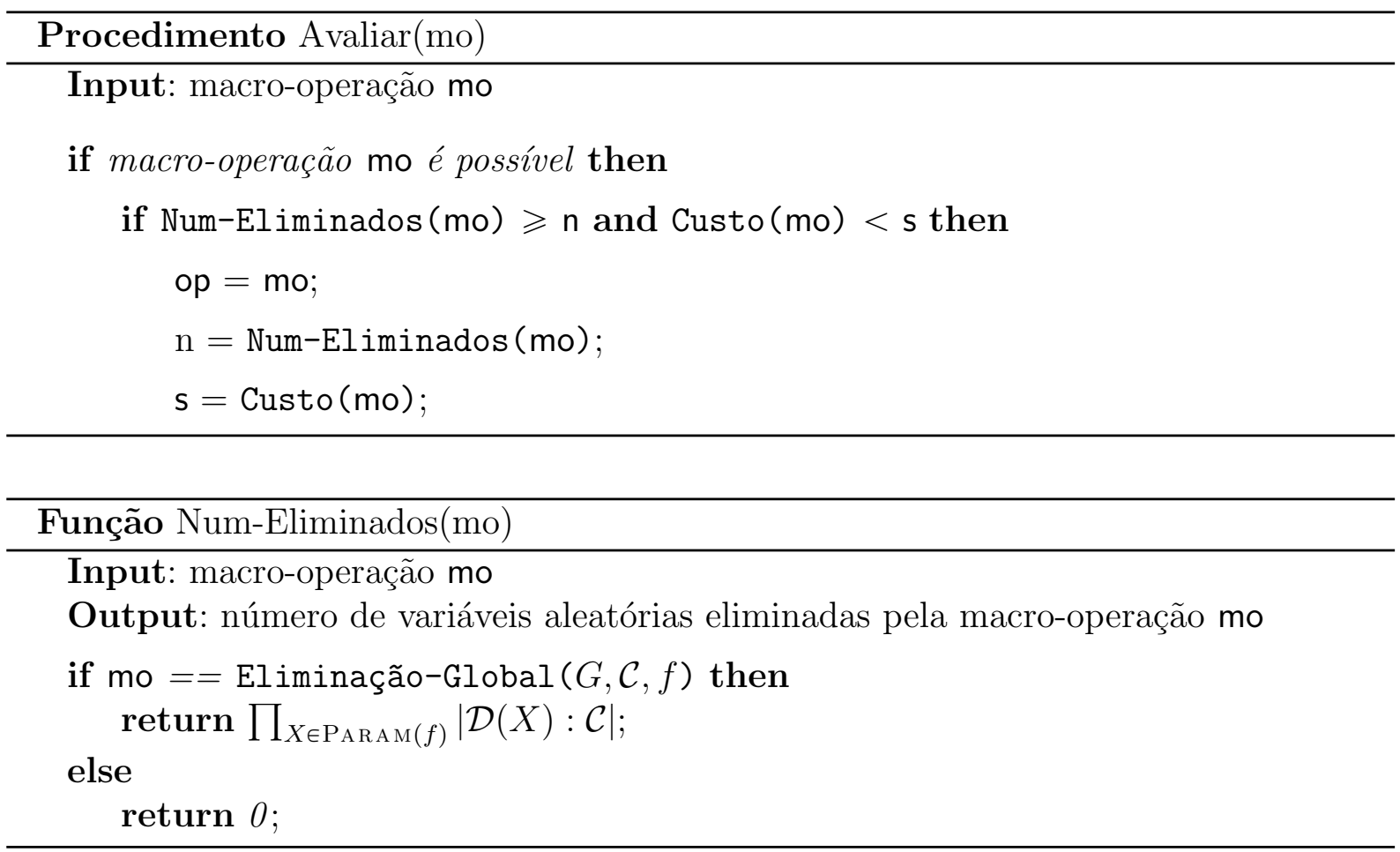

Exemplo 44. Neste exemplo será apresentada uma inferência completa utilizando o algoritmo AC-FOVE. Para isso, será utilizada a rede Loteria, proposta por (KISYNSKI, 2010) e reproduzida na Figura \%. Nesta rede, relacionam-se as probabilidades da loteria oferecer um prêmio excepcionalmente grande, de uma pessoa apostar e acertar 6 números e de alguém ganhar o prêmio. A variável lógica $P$ está atrelada a uma população de 10 pessoas $\left\{x_{1}, x_{2}, \ldots, x_{10}\right\}$.

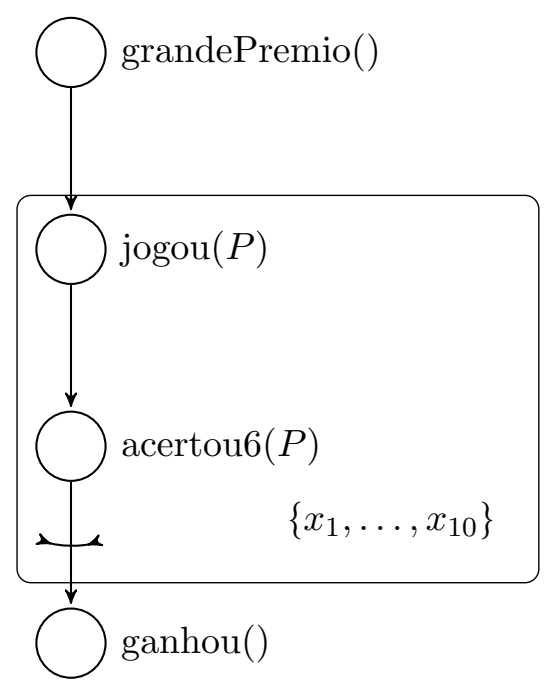

Figura 7: Rede Loteria proposta por Kisynski (2010)

Neste exemplo, não serão explicitados os fatores dos parfactors durante a computação. 


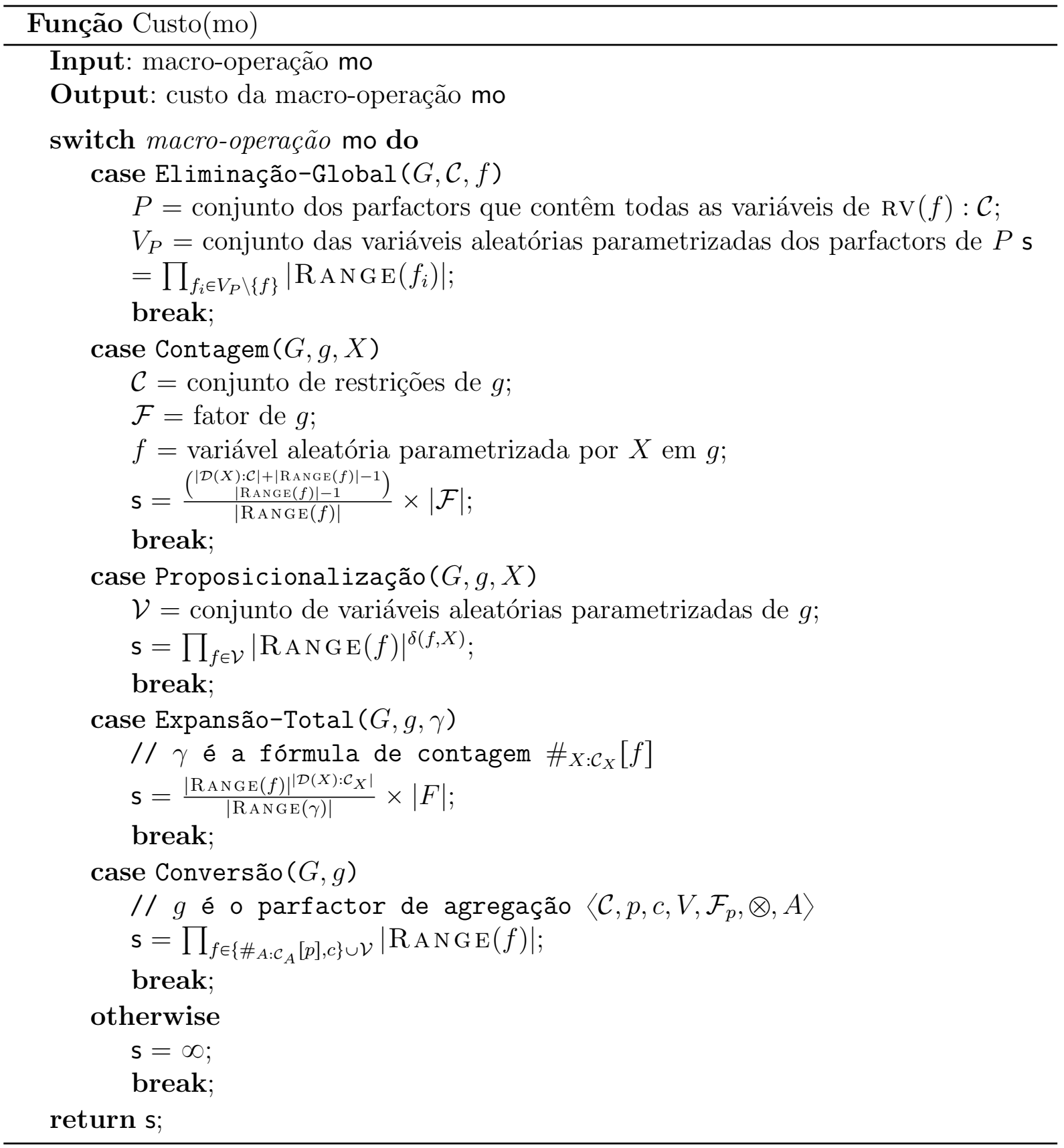


Os parfactors correspondentes à rede da Figura 7 são dados pelo seguinte conjunto:

$$
\begin{array}{rlr}
G_{0}= & \left\langle\left\langle\varnothing,\{\text { grandePremio }()\}, \mathcal{F}_{1}\right\rangle,\right. & \left(g_{1}\right) \\
& \left\langle\varnothing,\{\text { grandePremio }(), \text { jogou }(P)\}, \mathcal{F}_{2}\right\rangle, & \left(g_{2}\right) \\
& \left\langle\varnothing,\{\text { jogou }(P), \operatorname{acertou} 6(P)\}, \mathcal{F}_{3}\right\rangle, & \left(g_{3}\right) \\
& \langle\varnothing, \operatorname{acertou} 6(P), \operatorname{ganhou}(), \varnothing, \mathbb{1}, \text { OR }, P\rangle\}
\end{array}
$$

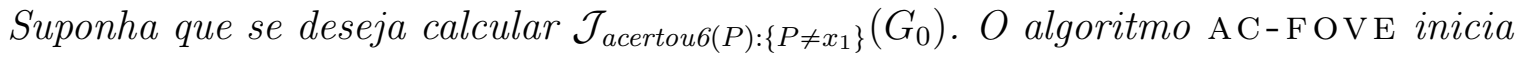
o procedimento particionando o conjunto $G_{0}$. O particionamento deve ser feito sobre a consulta também. Assim, os parfactors $g_{3}$ e $g_{4}$ precisam ser divididos em $\left\{P / x_{1}\right\}$. Para manter os conjuntos de variáveis aleatórias consistentes entre os parfactors, o parfactor $g_{2}$ também será dividido em $\left\{P / x_{1}\right\}$. Obtém-se o seguinte conjunto de parfactors:

$$
\begin{aligned}
G_{1}= & \left\langle\varnothing \varnothing,\{\text { grandePremio }()\}, \mathcal{F}_{1}\right\rangle, \\
& \left\langle\varnothing,\left\{\text { grandePremio }(), \text { jogou }\left(x_{1}\right)\right\}, \mathcal{F}_{2}\right\rangle, \\
& \left\langle\left\{P \neq x_{1}\right\},\{\text { grandePremio }(), \text { jogou }(P)\}, \mathcal{F}_{2}\right\rangle, \\
& \left\langle\varnothing,\left\{\text { jogou }\left(x_{1}\right), \operatorname{acertou} 6\left(x_{1}\right)\right\}, \mathcal{F}_{3}\right\rangle, \\
& \left\langle\left\{P \neq x_{1}\right\},\{\text { jogou }(P), \operatorname{acertou} 6(P)\}, \mathcal{F}_{3}\right\rangle, \\
& \left\langle\left\{P \neq x_{1}\right\}, \operatorname{acertou} 6(P), \text { ganhou }^{\prime}(), \varnothing, \mathbb{1}, \text { oR }, P\right\rangle, \\
& \left\langle\varnothing,\left\{\text { acertou}(P), g_{6}\right)\right.
\end{aligned}
$$

O fator $\mathcal{F}_{10}$ é obtido segundo o procedimento da Proposição 10. O objetivo do AC-F OVE neste exemplo é obter um parfactor nas variáveis $\mathrm{RV}($ acertou $6(P)):\left\{P \neq x_{1}\right\}$. Para tanto, os seguintes conjuntos de variáveis aleatórias serão eliminados:

- $\operatorname{RV}($ grandePremio ()$)$;

- $\operatorname{RV}\left(j o g o u\left(x_{1}\right)\right)$;

- $\operatorname{RV}(j o g o u(P)):\left\{P \neq x_{1}\right\}$

- $\operatorname{RV}\left(\operatorname{acertou} 6\left(x_{1}\right)\right)$;

- $\operatorname{RV}($ ganhou’());

- $\operatorname{RV}(\operatorname{ganhou}())$.

Após o particionamento inicial, o AC-FOVE deve ponderar entre as seguintes macrooperações: 
- EliminaÇÃo-Global $\left(G_{1}, j o g o u\left(x_{1}\right), \varnothing\right)$ : elimina 1 variável aleatória e tem custo 4 .

- EliminaÇÃo-Global $\left(G_{1}\right.$, ganhou ()$\left., \varnothing\right)$ : elimina 1 variável aleatória e tem custo 4 .

- EliminaÇÃ O-Global $\left(G_{1}, j o g o u(P),\left\{P \neq x_{1}\right\}\right)$ : elimina 9 variáveis aleatórias e tem custo 4 .

- EliminaÇÃ̃-Global $\left(G_{1}\right.$, acertou $\left.6\left(x_{1}\right), \varnothing\right)$ : elimina 1 variável aleatória e tem custo 8.

- Conversã o $\left(G_{1}, g_{9}\right)$ : não elimina variáveis aleatórias e tem custo 20.

- Contagem $\left(G_{1}, g_{6}, P\right)$ : não elimina variáveis aleatórias e tem custo 20.

As três primeiras operações possuem o mesmo custo, entretanto a terceira elimina mais variáveis e por isso é escolhida. A operação multiplica os parfactors que envolvem as variáveis do conjunto $\operatorname{RV}(j o g o u(P)):\left\{P \neq x_{1}\right\}$ e em seguida elimina a variável jogou $(P)$ do resultado. O parfactor resultante é dado por

$$
\sum_{\operatorname{Rv}(\text { jogou }(P)):\left\{P \neq x_{1}\right\}} g_{6} \odot g_{8}=\left\langle\left\{P \neq x_{1}\right\},\{\text { grandePremio }(), \operatorname{acertou} 6(P)\}, \mathcal{F}_{11}\right\rangle \quad\left(g_{11}\right)
$$

em que $\mathcal{F}_{11}=\sum_{\text {jogou(P) }} \mathcal{F}_{2} \odot \mathcal{F}_{3}$. O novo conjunto de parfactors será

$$
\begin{aligned}
G_{2}= & \left\{\left\langle\varnothing,\{\text { grandePremio }()\}, \mathcal{F}_{1}\right\rangle,\right. \\
& \left\langle\varnothing,\left\{\text { grandePremio }(), \text { jogou }\left(x_{1}\right)\right\}, \mathcal{F}_{2}\right\rangle, \\
& \left\langle\varnothing,\left\{\text { jogou }\left(x_{1}\right), \operatorname{acertou} 6\left(x_{1}\right)\right\}, \mathcal{F}_{3}\right\rangle, \\
& \left\langle\left\{P \neq x_{1}\right\}, \text { acertou } 6(P), \text { ganhou' }(), \varnothing, \mathbb{1}, \text { o R }, P\right\rangle, \\
& \left\langle\varnothing,\{\text { acertou } 6(P), \text { ganhou' }(), \text { ganhou }()\}, \mathcal{F}_{10}\right\rangle, \\
& \left.\left\langle\left\{P \neq x_{1}\right\},\{\text { grandePremio }(), \operatorname{acertou} 6(P)\}, \mathcal{F}_{11}\right\rangle\right\}
\end{aligned}
$$

O AC-FOVE realiza em seguida um novo levantamento das possíveis operações:

- EliminaÇão-Global $\left(G_{2}, j o g o u\left(x_{1}\right), \varnothing\right)$ : elimina 1 variável aleatória e tem custo 4 .

- EliminaÇÃo-Global $\left(G_{2}\right.$, ganhou ()$\left., \varnothing\right)$ : elimina 1 variável aleatória e tem custo 4. 
- Elimina Çã O-Global $\left(G_{2}\right.$, acertou $\left.6\left(x_{1}\right), \varnothing\right)$ : elimina 1 variável aleatória e tem custo 8.

- Convers Ã $\left(G_{2}, g_{9}\right)$ : não elimina variáveis aleatórias e tem custo 20.

- Contagem $\left(G_{2}, g_{11}, P\right)$ : não elimina variáveis aleatórias e tem custo 20.

As duas primeiras macro-operações tem custos iguais e eliminam o mesmo número de variáveis. Suponha que o AC-FOVE escolhe aleatoriamente a segunda operação. $O$ parfactor resultante é dado por

$$
\sum_{\operatorname{Rv}(\text { ganhou }()): \varnothing} g_{10}=\left\langle\varnothing,\left\{\operatorname{acertou} 6\left(x_{1}\right), \text { ganhou }^{\prime}()\right\}, \mathcal{F}_{12}\right\rangle
$$

em que $\mathcal{F}_{12}=\sum_{\text {ganhou( })} \mathcal{F}_{10}$. O novo conjunto de parfactors será

$$
\begin{aligned}
G_{3}= & \left\{\left\langle\varnothing,\{\text { grandePremio }()\}, \mathcal{F}_{1}\right\rangle,\right. \\
& \left\langle\varnothing,\left\{\text { grandePremio }(), \text { jogou }\left(x_{1}\right)\right\}, \mathcal{F}_{2}\right\rangle, \\
& \left\langle\varnothing,\left\{\text { jogou }\left(x_{1}\right), \operatorname{acertou} 6\left(x_{1}\right)\right\}, \mathcal{F}_{3}\right\rangle, \\
& \left\langle\left\{P \neq x_{1}\right\}, \text { acertou } 6(P), \text { ganhou }(), \varnothing, \mathbb{1}, \text { oR }, P\right\rangle, \\
& \left\langle\left\{P \neq x_{1}\right\},\{\text { grandePremio }(), \text { acertou } 6(P)\}, \mathcal{F}_{11}\right\rangle, \\
& \left.\left\langle\varnothing,\left\{\text { acertou6 }\left(x_{1}\right), \text { ganhou }^{\prime}()\right\}, \mathcal{F}_{12}\right\rangle\right\}
\end{aligned}
$$

O ciclo se inicia novamente e as operações disponíveis são:

- Elimina Çã O-GL obal $\left(G_{3}\right.$, acertou $\left.6\left(x_{1}\right), \varnothing\right)$ : elimina 1 variável aleatória e tem custo 4 .

- EliminaÇão-Global $\left(G_{3}, j o g o u\left(x_{1}\right), \varnothing\right)$ : elimina 1 variável aleatória e tem custo 4 .

- Convers Ã $\left(G_{3}, g_{9}\right)$ : não elimina variáveis aleatórias e tem custo 20.

- Contagem $\left(G_{3}, g_{11}, P\right)$ : não elimina variáveis aleatórias e tem custo 20.

As duas primeira operações tem custos iguais e eliminam o mesmo número de variáveis. Suponha que o AC-FOVE escolheu a primeira operação. O parfactor resultante será

$$
\sum_{\operatorname{Rv}\left(\text { acertou } 6\left(x_{1}\right)\right): \varnothing} g_{7} \odot g_{12}=\left\langle\varnothing,\left\{\text { jogou }\left(x_{1}\right), \text { ganhou }^{\prime}()\right\}, \mathcal{F}_{13}\right\rangle
$$


em que $\mathcal{F}_{13}=\sum_{\text {acertou6(x }\left(x_{1}\right)} \mathcal{F}_{3} \odot \mathcal{F}_{12}$. O conjunto de parfactors se torna

$$
\begin{aligned}
G_{4}= & \left\{\left\langle\varnothing,\{\text { grandePremio }()\}, \mathcal{F}_{1}\right\rangle,\right. \\
& \left\langle\varnothing,\left\{\text { grandePremio }(), \text { jogou }\left(x_{1}\right)\right\}, \mathcal{F}_{2}\right\rangle, \\
& \left\langle\left\{P \neq x_{1}\right\}, \operatorname{acertou} 6(P), \text { ganhou' }(), \varnothing, \mathbb{1}, \text { OR }, P\right\rangle, \\
& \left\langle\left\{P \neq x_{1}\right\},\{\text { grandePremio }(), \text { acertou } 6(P)\}, \mathcal{F}_{11}\right\rangle, \\
& \left.\left\langle\varnothing,\left\{\text { jogou }\left(x_{1}\right), \text { ganhou' }()\right\}, \mathcal{F}_{13}\right\rangle\right\}
\end{aligned}
$$

Desta vez, as operações disponíveis são:

- EliminaÇÃo-Global $\left(G_{4}, j o g o u\left(x_{1}\right), \varnothing\right)$ : elimina 1 variável aleatória e tem custo 4 .

- Convers Ã $\left(G_{4}, g_{9}\right)$ : não elimina variáveis aleatórias e tem custo 20.

- Contagem $\left(G_{4}, g_{11}, P\right)$ : não elimina variáveis aleatórias e tem custo 20.

O AC-FOVE escolhe a primeira operação. O resultado será

$$
\sum_{\operatorname{Rv}\left(\text { jogou }\left(x_{1}\right)\right): \varnothing} g_{5} \odot g_{13}=\left\langle\varnothing,\{\text { grandePremio }(), \text { ganhou' }()\}, \mathcal{F}_{14}\right\rangle
$$

em que $\mathcal{F}_{14}=\sum_{\text {acertou6(x) }} \mathcal{F}_{2} \odot \mathcal{F}_{13}$. O conjunto de parfactors se reduz a

$$
\begin{aligned}
G_{5}= & \left\langle\left\langle\varnothing,\{\text { grandePremio }()\}, \mathcal{F}_{1}\right\rangle,\right. \\
& \left\langle\left\{P \neq x_{1}\right\}, \text { acertou } 6(P), \text { ganhou }(), \varnothing, \mathbb{1}, \text { o R }, P\right\rangle, \\
& \left\langle\left\{P \neq x_{1}\right\},\{\text { grandePremio }(), \text { acertou } 6(P)\}, \mathcal{F}_{11}\right\rangle, \\
& \left.\left\langle\varnothing,\{\text { grandePremio }(), \text { ganhou' }()\}, \mathcal{F}_{14}\right\rangle\right\}
\end{aligned}
$$

Restam duas operações possíveis:

- Convers Ã $\left(G_{5}, g_{9}\right)$ : não elimina variáveis aleatórias e tem custo 20.

- Contagem $\left(G_{5}, g_{11}, P\right)$ : não elimina variáveis aleatórias e tem custo 20.

Suponha que o AC-FOVE escolha a segunda operação. Após a contagem, o parfactor $g_{11}$ se torna

$$
\left\langle\varnothing,\left\{\text { grandePremio }(), \#_{P:\left\{P \neq x_{1}\right\}}[\operatorname{acertou} 6(P)]\right\}, \mathcal{F}_{15}\right\rangle
$$


em que $\mathcal{F}_{15}$ é calculado segundo a Proposição 13. O conjunto $G_{5}$ se torna

$$
\begin{aligned}
G_{6}=\{ & \left\langle\varnothing,\{\text { grandePremio }()\}, \mathcal{F}_{1}\right\rangle, \\
& \left\langle\left\{P \neq x_{1}\right\}, \operatorname{acertou} 6(P), \text { ganhou }(), \varnothing, \mathbb{1}, \text { o R }, P\right\rangle, \\
& \left\langle\left\{P \neq x_{1}\right\},\{\text { grandePremio }(), \operatorname{acertou} 6(P)\}, \mathcal{F}_{11}\right\rangle, \\
& \left.\left\langle\varnothing,\left\{\text { grandePremio }(), \#_{P:\left\{P \neq x_{1}\right\}}[\operatorname{acertou} 6(P)]\right\}, \mathcal{F}_{15}\right\rangle\right\}
\end{aligned}
$$

A operação de contagem abre uma nova possibilidade para o algoritmo:

- Convers Ã o $\left(G_{6}, g_{9}\right)$ : não elimina variáveis aleatórias e tem custo 20.

- Elimina Ção-Global $\left(G_{6}\right.$, grandePremio ()$\left., \varnothing\right)$ : elimina 1 variável aleatória e tem custo 20.

A segunda operação é escolhida, pois elimina uma variável aleatória. O resultado desta operação será

$$
\sum_{\operatorname{Rv}(\text { grandePremio }()): \varnothing} g_{1} \odot g_{14} \odot g_{15}=\left\langle\varnothing,\left\{\text { ganhou }^{\prime}(), \#_{P:\left\{P \neq x_{1}\right\}}[\operatorname{acertou} 6(P)]\right\}, \mathcal{F}_{16}\right\rangle \quad\left(g_{16}\right)
$$

em que $\mathcal{F}_{16}=\sum_{\text {grandePremio }()} \mathcal{F}_{1} \odot \mathcal{F}_{14} \odot \mathcal{F}_{15}$. O conjunto de parfactors após a eliminação fica reduzido a

$$
\begin{aligned}
G_{7}=\{ & \left\langle\left\{P \neq x_{1}\right\}, \text { acertou } 6(P), \text { ganhou }^{\prime}(), \varnothing, \mathbb{1}, \text { OR }, P\right\rangle, \\
& \left.\left\langle\varnothing,\left\{\text { ganhou' }^{\prime}(), \#_{P:\left\{P \neq x_{1}\right\}}[\operatorname{acertou} 6(P)]\right\}, \mathcal{F}_{16}\right\rangle\right\}
\end{aligned}
$$

Resta agora uma operação disponível:

- Convers Ã $\left(G_{7}, g_{9}\right)$ : não elimina variáveis aleatórias e tem custo 20.

A conversão de $g_{9}$ para parfactors na forma padrão resulta em dois parfactors conforme a Proposição 12. O conjunto de parfactors se torna

$$
\begin{aligned}
G_{8}= & \left\langle\left\langle\varnothing,\left\{\text { ganhou' }^{\prime}(), \#_{P:\left\{P \neq x_{1}\right\}}[\operatorname{acertou} 6(P)]\right\}, \mathcal{F}_{16}\right\rangle,\right. \\
& \left\langle\left\{P \neq x_{1}\right\},\{\text { acertou } 6(P)\}, \mathbb{1}\right\rangle,
\end{aligned}
$$

Note que, se for feita a contagem de $P$ em $g_{17}$, o resultado será um parfactor na variável aleatória parametrizada $\#_{P:\left\{P \neq x_{1}\right\}}[\operatorname{acertou} 6(P)]$, que por sua vez poderá ser multiplicado 
pelos demais parfactors do conjunto. Entretanto, como o fator de $g_{17}$ é constante e unitário, a multiplicação não alterará os demais parfactors. Desse modo, pode-se remover o parfactor $g_{17}$ sem que o resultado final seja alterado:

$$
\begin{aligned}
G_{9}= & \left\{\left\langle\varnothing,\left\{\text { ganhou }^{\prime}(), \#_{P:\left\{P \neq x_{1}\right\}}[\operatorname{acertou} 6(P)]\right\}, \mathcal{F}_{16}\right\rangle,\right. \\
& \left.\left\langle\varnothing,\left\{\#_{P:\left\{P \neq x_{1}\right\}}\left[\operatorname{acertou}_{6}(P)\right], \text { ganhou }^{\prime}()\right\}, \mathcal{F}_{18}\right\rangle\right\}
\end{aligned}
$$

Novamente, é possível realizar apenas uma operação neste caso:

- EliminaÇÃo-Global $\left(G_{9}\right.$, ganhou'( $\left.), \varnothing\right)$ : elimina 1 variável aleatória e tem custo 10 .

A operação resulta no seguinte parfactor:

$$
\sum_{\operatorname{Rv}\left(\text { ganhou }^{\prime}()\right): \varnothing} g_{16} \odot g_{18}=\left\langle\varnothing,\left\{\#_{P:\left\{P \neq x_{1}\right\}}[\operatorname{acertou} 6(P)]\right\}, \mathcal{F}_{19}\right\rangle
$$

em que $\mathcal{F}_{19}=\sum_{\text {acertoub(x }\left(x_{1}\right)} \mathcal{F}_{16} \odot \mathcal{F}_{18}$. O conjunto final de parfactors será

$$
G_{10}=\left\{\left\langle\varnothing,\left\{\#_{P:\left\{P \neq x_{1}\right\}}[\operatorname{acertou} 6(P)]\right\}, \mathcal{F}_{19}\right\rangle\right\}
$$

Tem-se finalmente

$$
\mathcal{J}_{\text {acertou } 6(P):\left\{P \neq x_{1}\right\}}\left(G_{0}\right)=\mathcal{J}\left(G_{10}\right)
$$

Note que, durante os cálculos, não foi necessário enumerar os indivíduos $\left\{x_{2}, \ldots, x_{10}\right\}$. Utilizou-se apenas o fato de que $|\mathcal{D}(P)|=10$ para calcular os custos. O maior fator criado durante a inferência tinha tamanho $2 \cdot\left(\begin{array}{c}2+9-1 \\ 2-1\end{array}\right)=20$. 


\section{Implementação}

A proposta deste trabalho é aplicar todos os conceitos e operações do algoritmo AC-F OVE para realizar inferências em redes de primeira ordem especificadas por meio de parfactors, através de um pacote de software que forneça as funcionalidades necessárias para a execução do algoritmo.

O pacote é fornecido na forma de uma biblioteca que pode ser utilizada em outros softwares e pode ser obtido a partir do repositório Git disponível em https ://github.com/ ftakiyama/AC-FOVE. Neste repositório encontram-se o código fonte da implementação e a documentação da utilização do pacote.

A linguagem de programação escolhida para a implementação é o Java, pelos seguintes motivos:

Multiplataforma $\mathrm{O}$ pacote não precisa ser compilado para plataformas e/ou sistemas operacionais específicos, o que facilita o seu uso e inclusão em outros pacotes de software;

Linguagem interpretada e de alto nível Permite criar maiores abstrações e acelerar o desenvolvimento;

API A Interface de Programação de Aplicativos da plataforma Java possui um série de estruturas de dados prontas para uso (tais como vetores, listas e árvores);

Documentação A popularidade da plataforma Java vem acompanhada de uma grande comunidade de desenvolvedores, o que ajuda na obtenção de material de referência. Além disso, a API Java é extensivamente documentada.

Embora programas em Java tenham, em geral, um desempenho menor que programas em linguagens compiladas (tais como $\mathrm{C} / \mathrm{C}++$, Haskell, Pascal etc.), as vantagens supracitadas sobrepujaram a questão do desempenho. 


\subsection{Metodologia}

A metodologia adotada para o projeto, a codificação e os testes do pacote foi o Extreme Programming (XP), uma metodologia de desenvolvimento ágil que possui como características:

- Programação em pares;

- Constante revisão do código;

- Desenvolvimento orientado a testes (TDD) (JANZEN; SAIEDIAN, 2005);

- Não inclusão de funcionalidades desnecessárias no momento;

- Simplicidade e clareza do código;

- Incorporação de mudanças nos requisitos à medida que o projeto avança;

- Comunicação frequente com clientes e entre desenvolvedores;

- Ênfase em um produto funcional em detrimento de documentação extensa.

A metodologia não foi seguida à risca devido à limitações de recursos e de escopo do projeto: não há um "cliente" que levanta os requisitos, nem uma "equipe" para coordenar. Foram incorporados ao desenvolvimento do projeto características de evolução incremental e pontual voltada a testes; isto é, para cada requisito tangível e suficientemente simples são desenvolvidos testes de unidade que certificam a funcionalidade desejável. O fluxograma da metodologia é exibido na Figura 8.

O início do projeto consistiu no levantamento dos requisitos desejáveis para o pacote de software. Em seguida é feito um planejamento em alto nível dos principais componentes do software que será desenvolvido. Para cada componente, é feito um projeto contemplando maiores detalhes daquilo que deve ser feito, para no próximo passo iniciar a codificação. Nesta fase, são desenvolvidos os testes de unidade e o componente. Este é refatorado até que seja "aprovado" em todos os testes unitários. Posteriormente o componente é integrado aos demais já desenvolvidos e testes gerais são conduzidos de maneira a observar (e eventualmente corrigir) o comportamento do sistema como um todo.

Com base nos resultados, novos requisitos são criados e/ou alterados, para dar início a um novo ciclo de desenvolvimento. As iterações prosseguem até que não haja mais funcionalidades a serem implementadas. 


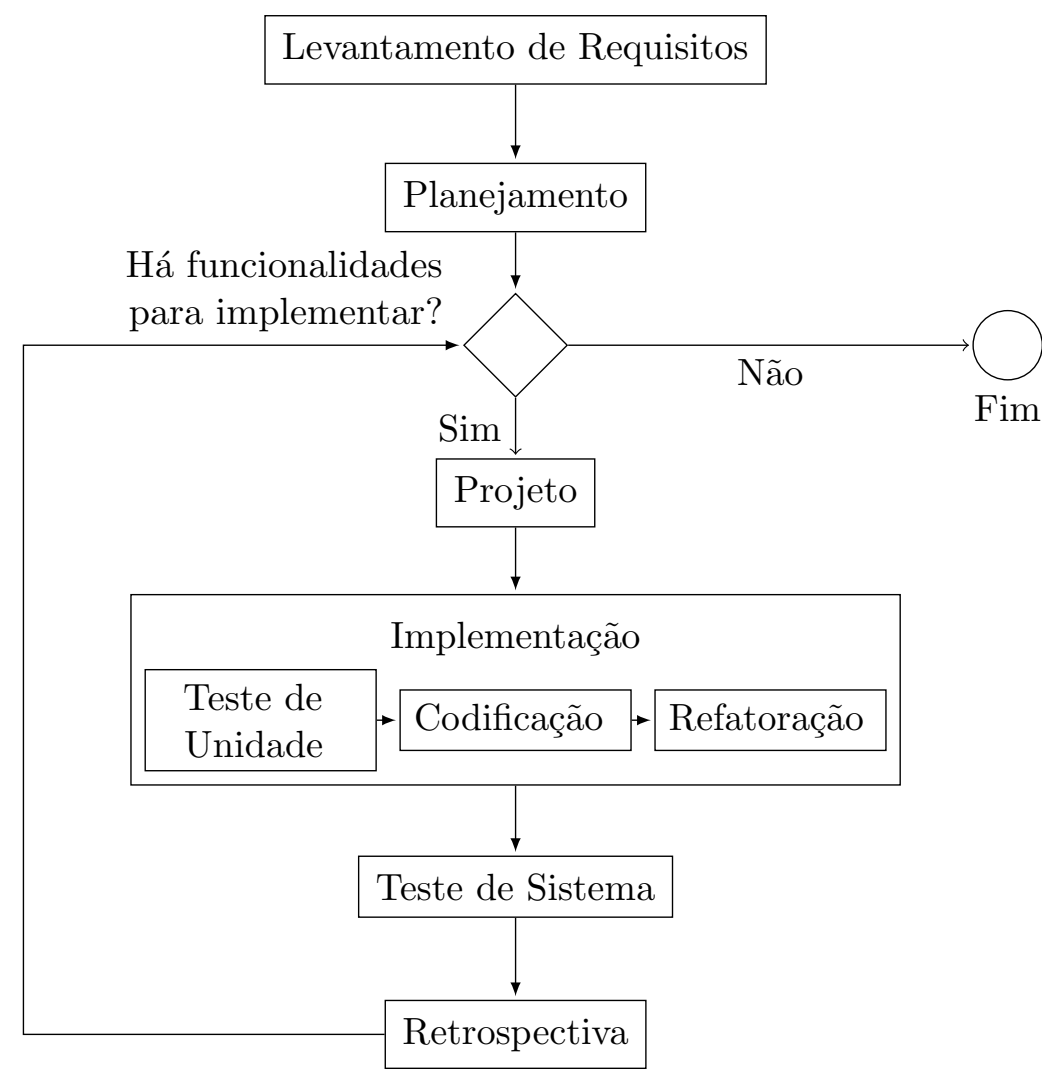

Figura 8: Metodologia XP (adaptado de (DZHUROV; KRASTEVA; ILIEVA, 2009))

\subsection{Fases do Projeto}

A implementação do algoritmo A C-F OVE foi dividida nas seguintes etapas:

VE A primeira etapa consiste no desenvolvimento do algoritmo de Eliminação de Variáveis (VE) utilizando uma heurística simples. A seleção da variável a ser eliminada é feita através de um esquema de filas (FIFO). Dado um conjunto de fatores que representa a distribuição de probabilidade de um conjunto de variáveis aleatórias, um conjunto de evidências e uma variável de consulta, o algoritmo retorna a distribuição a posteriori nesta variável.

C-FOVE A segunda etapa consiste no desenvolvimento do algoritmo de Eliminação de Variáveis em Primeira Ordem. Nesta fase do projeto são implementadas as estruturas de dados relevantes, tais como parfactors e variáveis aleatórias parametrizadas, bem como as operações elementares de manipulação destas estruturas e as macrooperações do algoritmo.

AC-FOVE A terceira etapa consiste em agregar ao C-F OVE os parfactors de agregação e adaptar as operações já existentes para tratar esta nova estrutura de dados. 


\subsection{Utilização}

O pacote de software criado é fornecido na forma de uma biblioteca de classes Java que permitem a criação de estruturas de dados necessárias para a execução do algoritmo A C-F OVE. Nesta seção serão mostrados trechos de código que elucidam a utilização das principais estruturas de dados fornecidas com o pacote.

\subsubsection{Definição de constantes}

Constantes são indivíduos de uma população e são representados por palavras que iniciam por um letra minúscula (POOLE; MACKWORTH, 2010). O trecho de código da Figura 9 ilustra um exemplo de como definir uma constante.

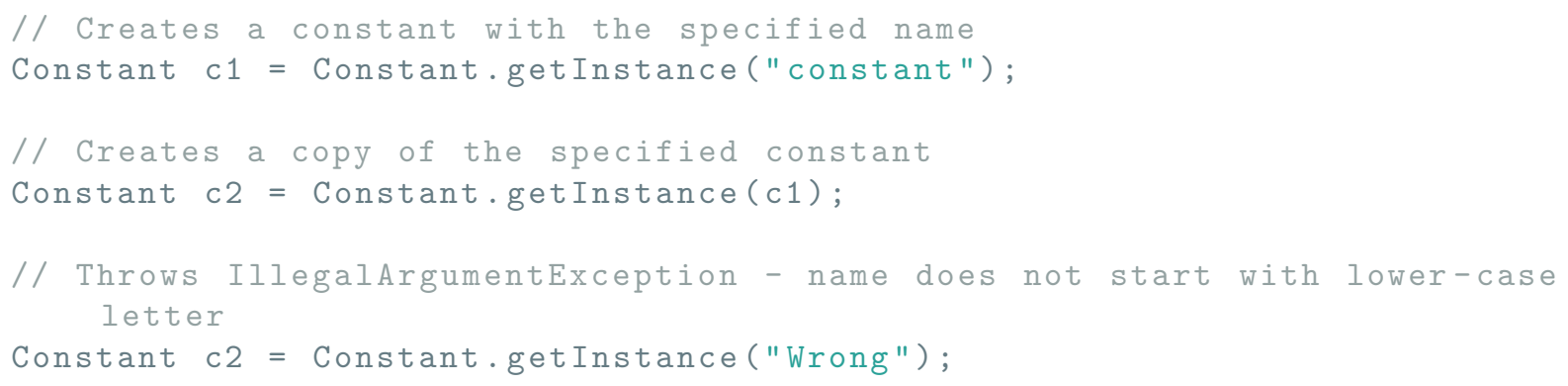

Figura 9: Código para definição de constante

\subsubsection{Definição de variáveis lógicas}

Variáveis lógicas são representadas por palavras iniciadas por letra maiúscula (POOLE; MACKWORTH, 2010) e estão atreladas a uma População de Constantes. O trecho de código da Figura 10 ilustra um exemplo de como definir uma variável lógica.

\subsubsection{Definição de restrições}

Restrições são desigualdades que envolvem duas Variáveis Lógicas ou uma Variável Lógica e uma Constante. O trecho de código da Figura 11 ilustra um exemplo de como definir uma restrição com duas variáveis lógicas e uma restrição com uma variável lógica e uma constante. 


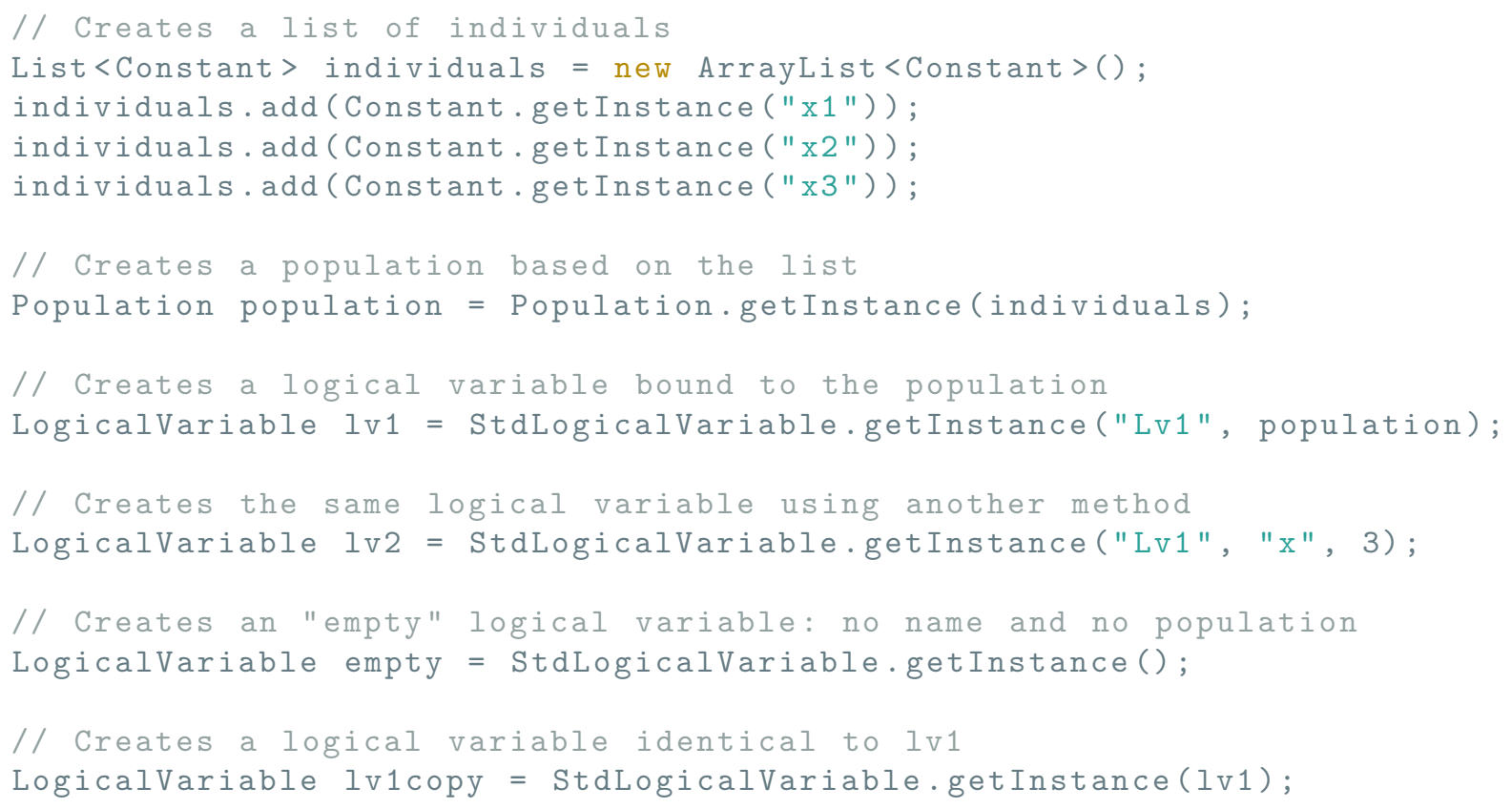

Figura 10: Código para definição de variável lógica

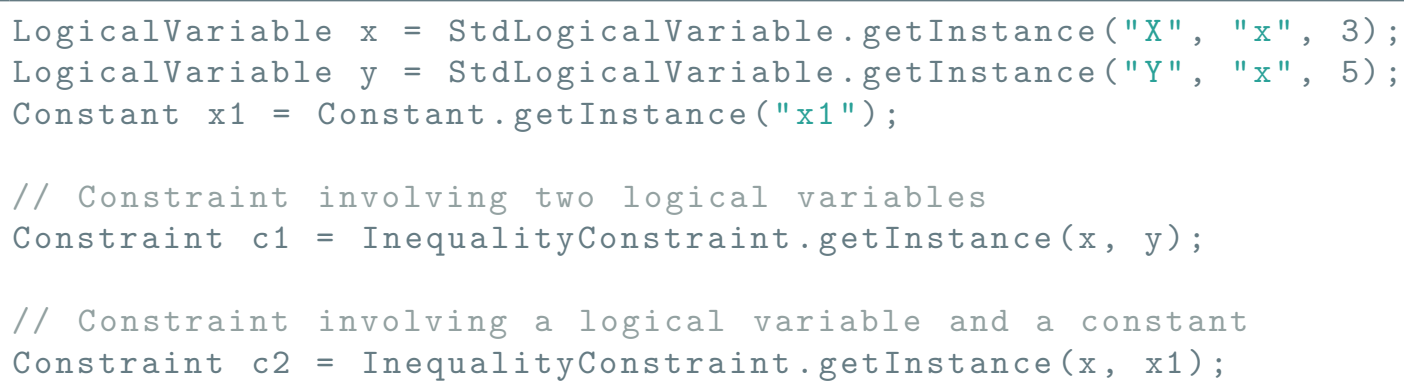

Figura 11: Código para definição de restrições

\subsubsection{Definição de variáveis aleatórias parametrizadas}

Variáveis aleatórias parametrizadas (PRVs) representam conjuntos de variáveis aleatórias. Nesta seção serão tratadas variáveis aleatórias parametrizadas na forma padrão.

A maneira mais genérica de definir uma variável aleatórias parametrizada na forma padrão é especificar seu nome (functor), seu domínio (valores que pode assumir) e seus termos (uma lista contendo variáveis lógicas e/ou constantes), conforme mostrado na Figura 12.

Domínios booleanos são muito comuns em variáveis aleatórias parametrizadas. Por este motivo, existe um método mais conveniente para criar tais variáveis. Esta alternativa é exibida na Figura 13. 


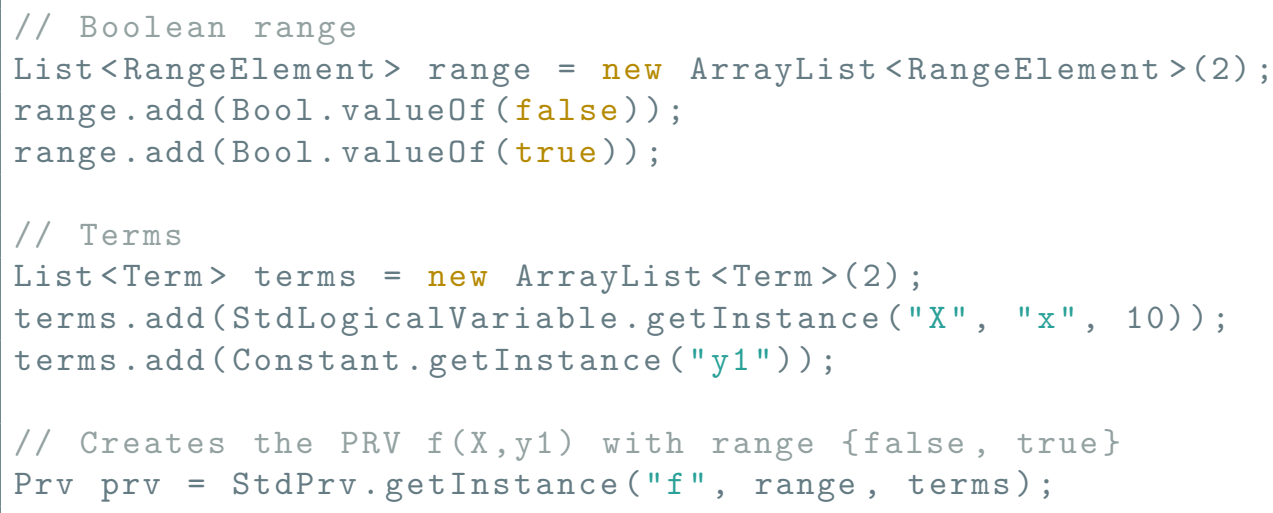

Figura 12: Código para definição de uma variável aleatória parametrizada na forma padrão

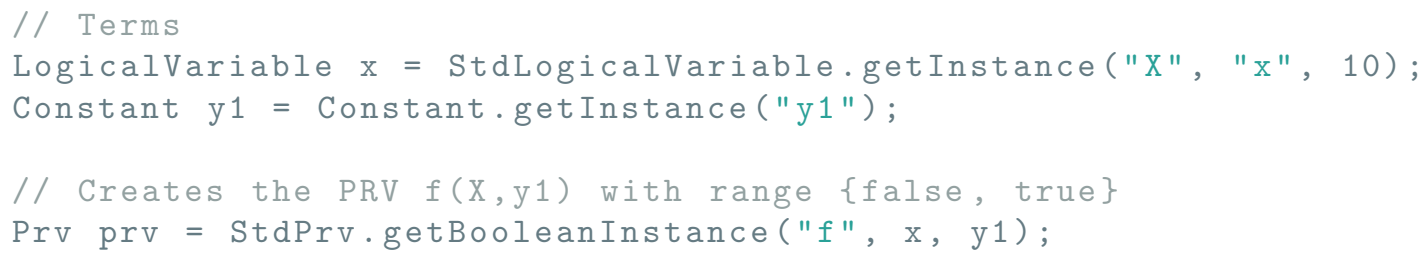

Figura 13: Código para definição de uma variável aleatória parametrizada booleana

\subsubsection{Definição de fórmulas de contagem}

Fórmulas de contagem são usadas para representar conjuntos de variáveis aleatórias quando é importante conhecer apenas o número de atribuições de valores às variáveis aleatórias representadas pelo conjunto. A definição de uma fórmula de contagem é dividida em três partes: definição de uma variável lógica atrelada, definição da variável aleatória parametrizada "contada" e definição de um conjunto de restrições de desigualdade envolvendo a variável lógica atrelada. O código da Figura 14 ilustra como esta definição é feita no pacote.

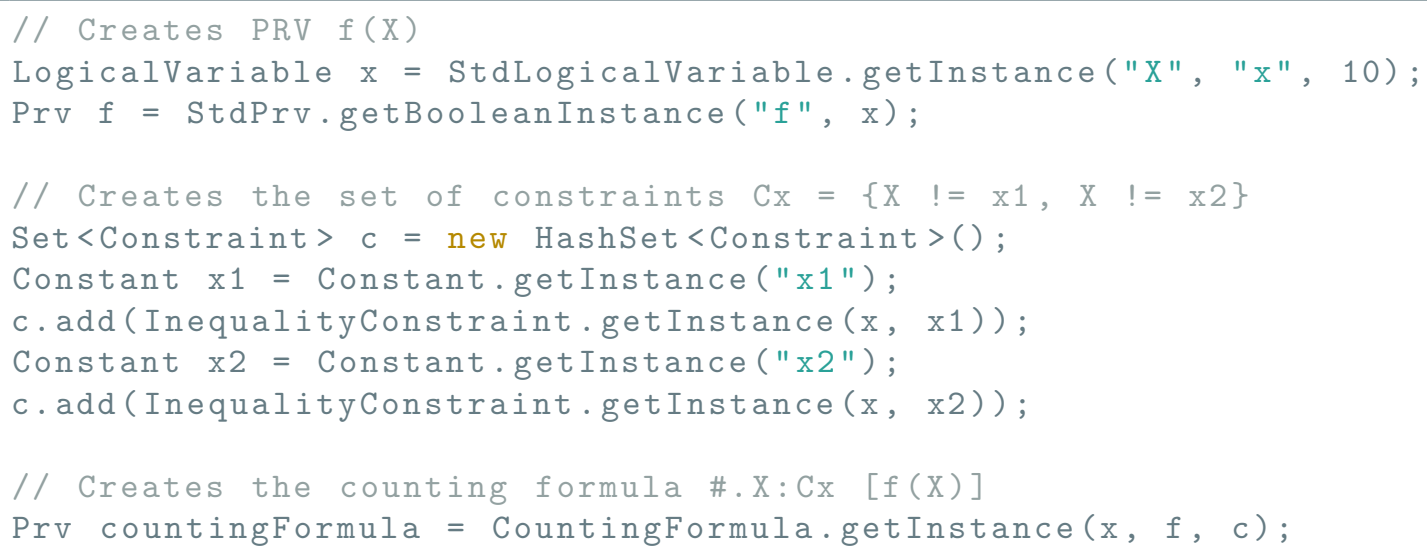

Figura 14: Código para definição de uma fórmula de contagem 
Uma limitação das fórmulas de contagem é que a variável aleatória parametrizada "contada" não pode ser uma outra fórmula de contagem.

\subsubsection{Definição de parfactors na forma padrão}

Um parfactor na forma padrão é composto de três partes: um conjunto de restrições, um conjunto de variáveis aleatórias parametrizadas e um fator. O fator representa a relação entre as variáveis aleatórias parametrizadas e é armazenado em um vetor de números reais. O código da Figura 15 exibe estas etapas na definição de um parfactor g1.

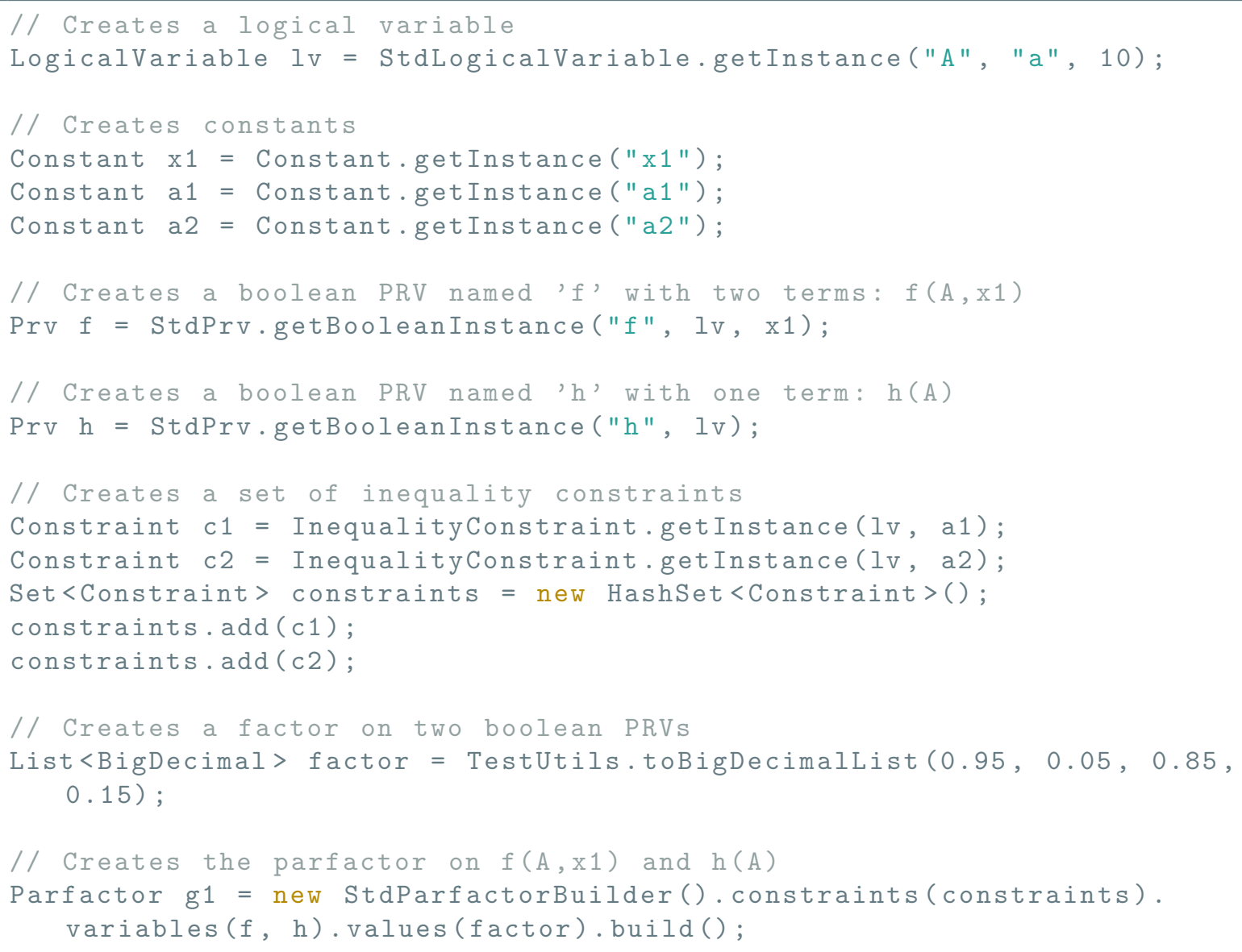

Figura 15: Código para definição de um parfactor 
O parfactor g1 representa o seguinte parfactor:

\begin{tabular}{cccc}
\cline { 2 - 4 }$\left\langle\left\{A \neq a_{1}, A \neq a_{2}\right\}\right.$, & $\mathrm{f}\left(A, x_{1}\right)$ & $\mathrm{h}(A)$ & \\
\cline { 2 - 4 } & $\mathrm{F}$ & $\mathrm{F}$ & 0.95 \\
$\mathrm{~F}$ & $\mathrm{~F}$ & 0.05 \\
& $\mathrm{~V}$ & $\mathrm{~V}$ & 0.85 \\
\hline
\end{tabular}

\subsubsection{Definição de parfactors de agregação}

Parfactor de agregação são usados para representar o efeito que ocorre quando um nó pai possui uma variável lógica que não aparece no nó filho. Ao proposicionalizar esta estrutura, o nó filho terá um número de pais que depende do número de indivíduos atrelados à população da variável lógica extra. Em inferência em primeira ordem, é necessário criar uma representação que não dependa deste número.

A agregação não pode ser representada por um parfactor na forma padrão. Ao invés disso, utiliza-se o parfactor de agregação, que possui seis componentes:

- Um conjunto de restrições;

- Uma variável aleatória parametrizada pai;

- Uma variável aleatória parametrizada filha;

- Um conjunto de variáveis aleatórias parametrizadas de contexto;

- Um fator;

- Um operador de agregação.

Apenas a variável pai, a variável filha e o operador de agregação são obrigatórios na definição de um parfactor de agregação, conforme ilustrado na Figura 16.

Os outros componentes podem ser adicionados conforme a necessidade. A Figura 17 mostra como adicionar restrições de desigualdade, variáveis aleatórias parametrizadas de contexto e valores diferentes de 1 no fator associado.

Ao criar parfactors de agregação para redes de primeira ordem, o fator deve sempre ser constante $(\mathbb{1})$. O fator representa a distribuição no pai e nas variáveis de contexto, mas 


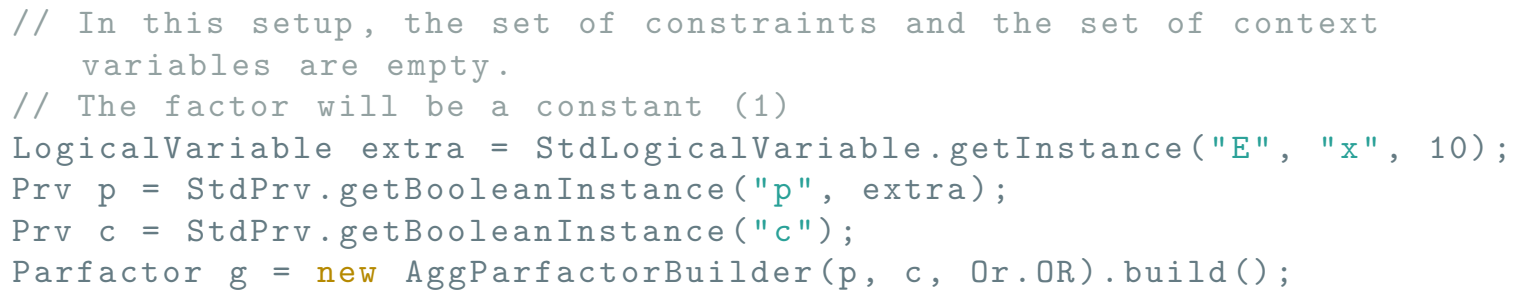

Figura 16: Código para definição de um parfactor de agregação simples

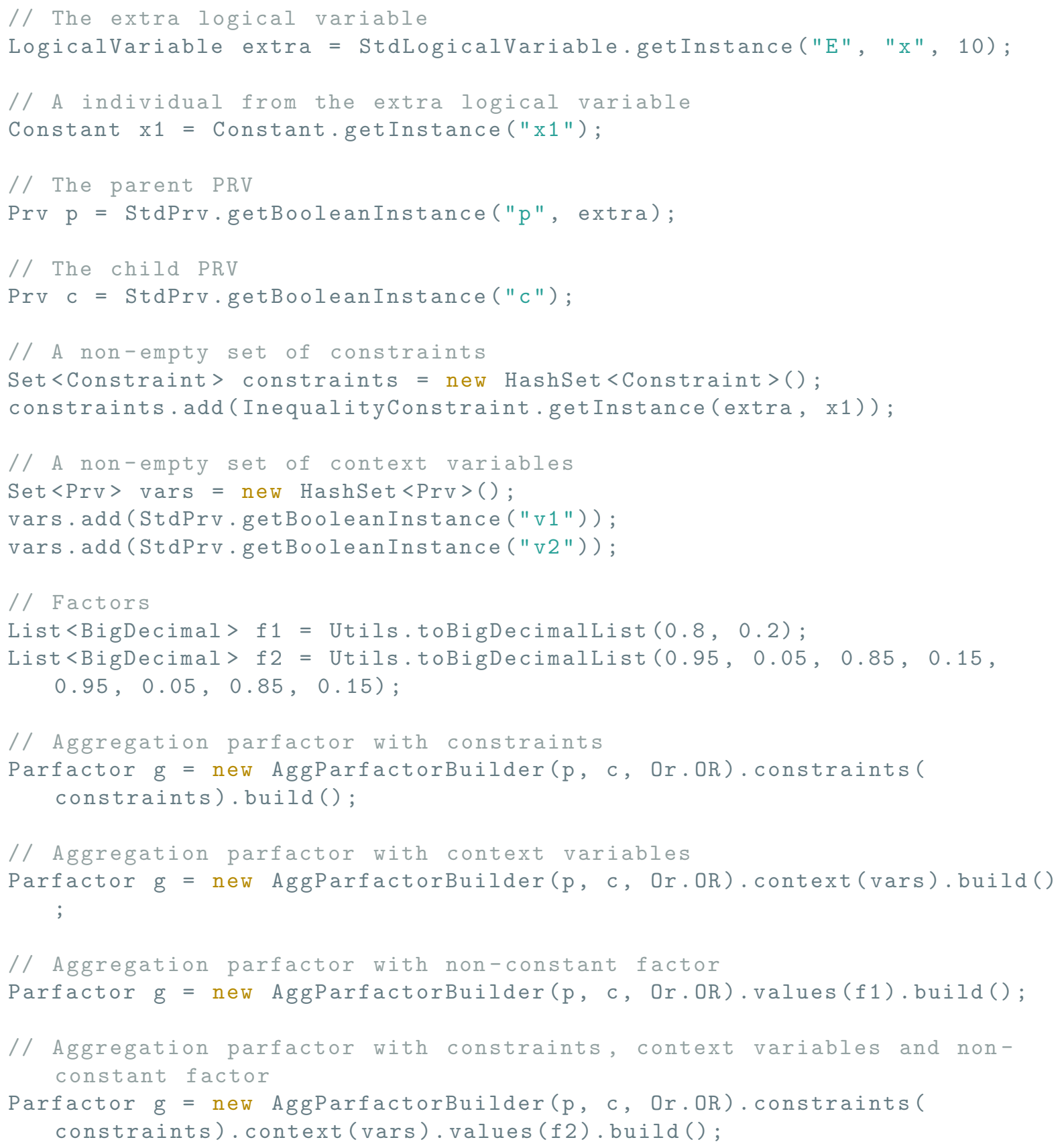

Figura 17: Código para definição de parfactors de agregação mais complexos 
quando a rede é "traduzida" para parfactors, esta distribuição já estará presente em um parfactor separado.

Parfactor de agregação não podem ser contados ou expandidos. O código da Figura 18 causará uma exceção em tempo de execução.

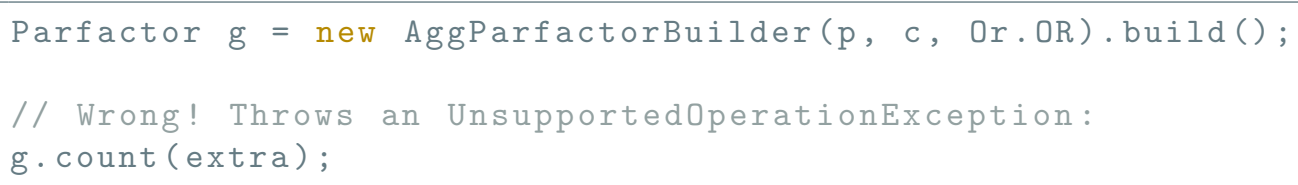

Figura 18: Chamada de método inválido para parfactor de agregação

Parfactors de agregação suportam divisões, multiplicações e eliminações sob condições específicas (explicadas em detalhes no Capítulo 3). O algoritmo AC-F OvE verifica estas condições ao realizar a inferência.

Parfactors de agregação devem ser atribuídos a variáveis do tipo Parfactor, mas em algumas situações pode ser necessários convertê-los para usar operações específicas. A Figura 19 exemplifica a maneira de "converter" um Parfactor em AggregationParfactor.

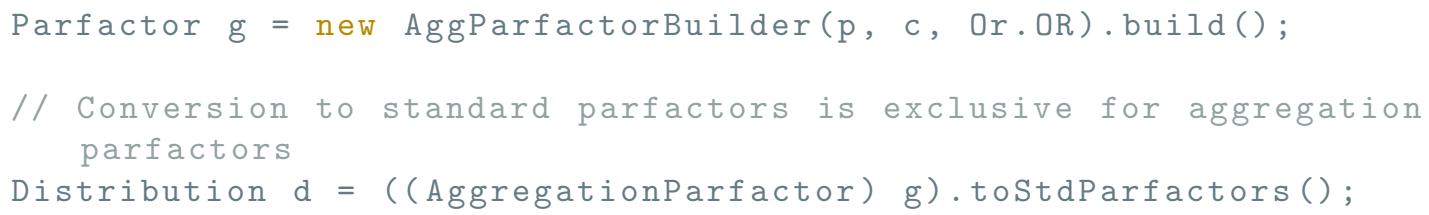

Figura 19: Código para converter um Parfactor em AggregationParfactor

\subsubsection{Definição de consulta}

Uma consulta é um conjunto de variáveis aleatórias. O A C-F OVE elimina as variáveis aleatórias que não pertencem a este conjunto e retorna a distribuição conjunta nas variáveis aleatórias da consulta.

A consulta é especificada por uma variável aleatória parametrizada padrão e um conjunto de desigualdades ${ }^{1}$. A Figura 20 mostra como definir a consulta $q=f\left(A, x_{1}\right)$ : $\{A \neq x 1, A \neq x 2\}$.

\footnotetext{
${ }^{1}$ No momento, esta é a única forma de definir a consulta.
} 


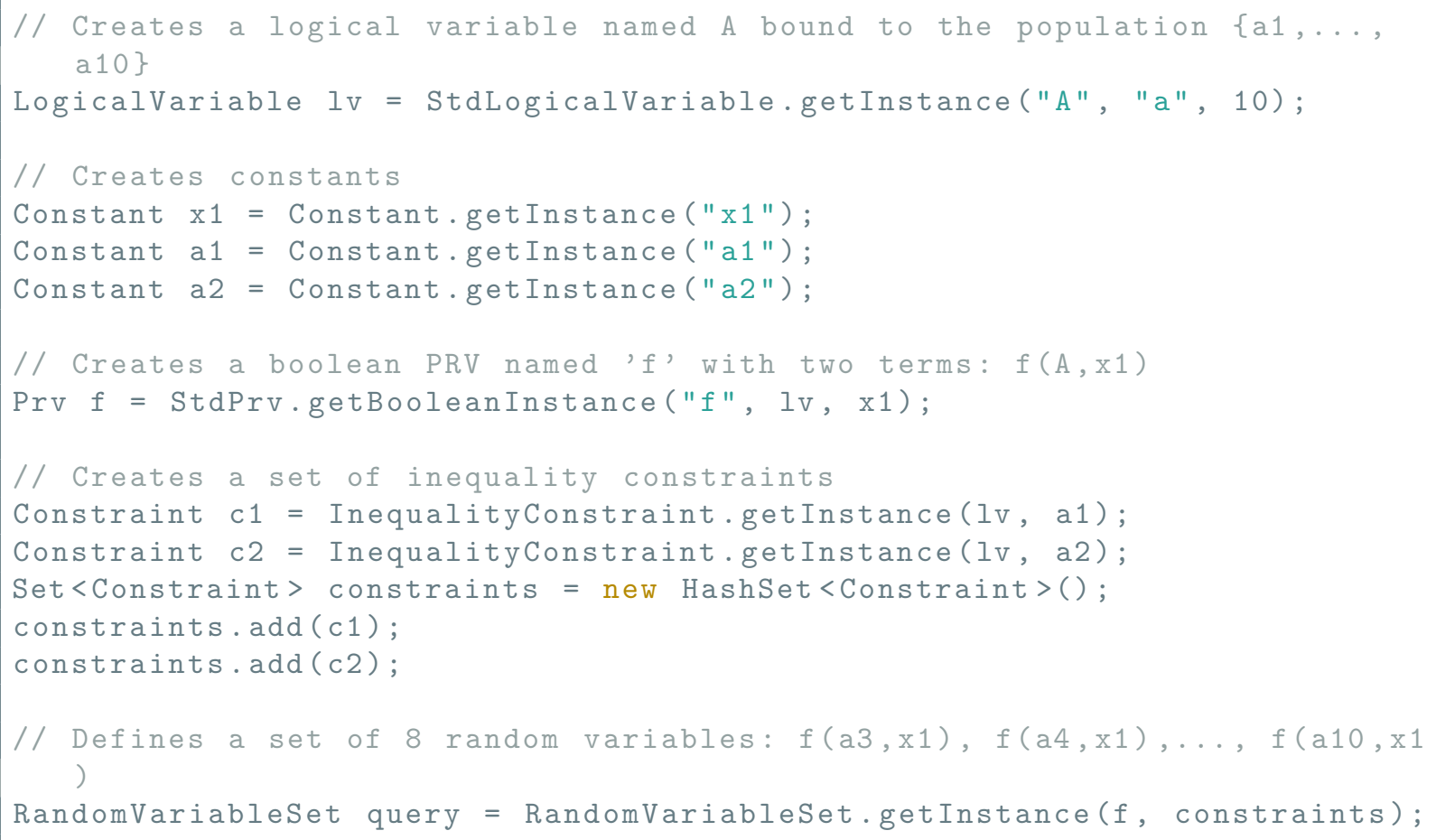

Figura 20: Código para definição de uma consulta para o A C-F OVE

\subsubsection{Definição de marginais}

O marginal representa a distribuição conjunta nas variáveis da consulta. Na Figura 21 exemplifica-se a montagem de um marginal.

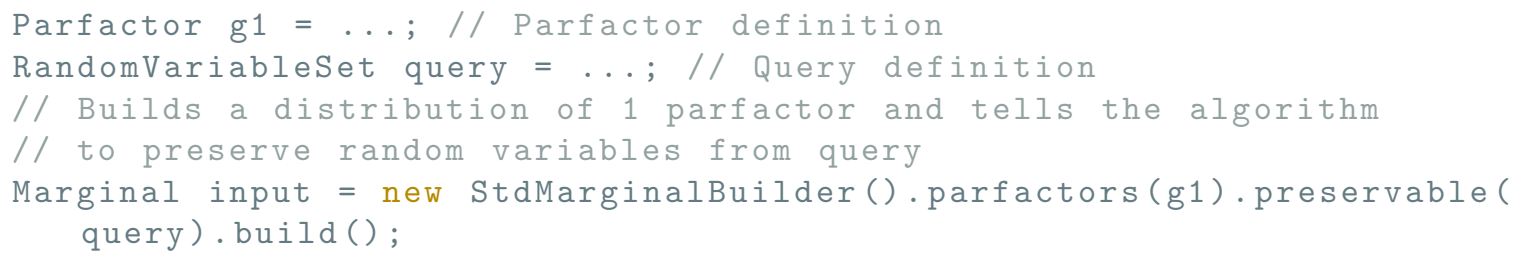

Figura 21: Código para definição de um margina

\subsubsection{Inclusão de evidências}

Evidências são fatos que foram observados antes da inferência e representam um conhecimento prévio acerca de um problema.

Evidências podem ser traduzidas diretamente para parfactors. Suponha que uma rede contenha quatro nós: nublado(), chuva(), sprinkler(Lote) e gramaMolhada(Lote). Considere que se sabe de antemão que o sprinkler do lote ${ }_{1}$ esteja ligado. Esta informação 
pode ser traduzida pelo parfactor

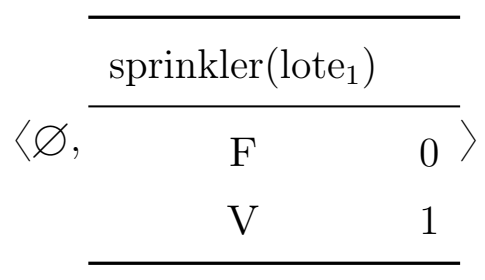

A Figura 22 mostra como evidências são criadas dentro do pacote.

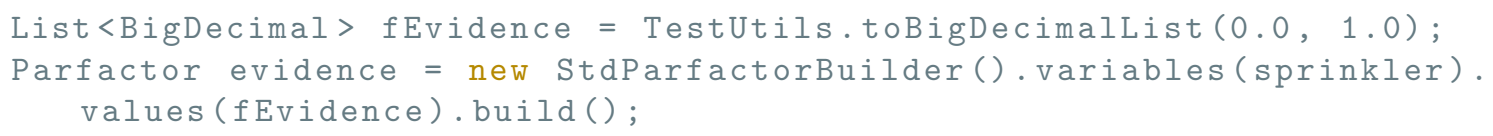

Figura 22: Código para definição de uma evidência

Este parfactor deve ser adicionado ao marginal juntamente com os demais parfactors.

\subsubsection{Execução do algoritmo}

O algoritmo A C-F OVE pode ser executado criando-se uma instância da classe ACFOVE. O trecho de código mostrado na Figura 23 exemplifica a chamada do algoritmo A C-F OVE.

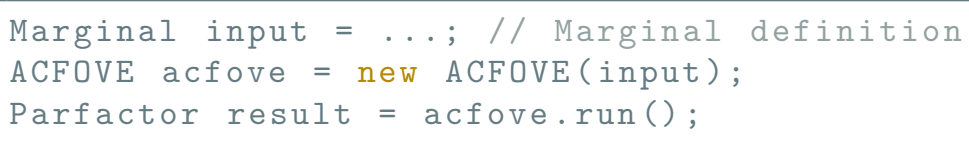

Figura 23: Código para executar o algoritmo A C-F OVE

O AC-FOVE retorna o parfactor que representa o marginal de um conjunto de parfactors em uma dada consulta. A título de exemplo, a Figura 24 lista uma especificação completa de uma rede de primeira ordem e a respectiva execução do algoritmo.

\subsubsection{Extensão do algoritmo}

O pacote de software foi desenvolvido de maneira que possa ser estendido conforme as necessidades. Isto foi possível devido ao uso de Interfaces, que permitem abstrair a implementação das assinaturas dos métodos. Cita-se a seguir uma lista com as principais interfaces do pacote, bem como uma breve descrição de cada uma.

Algorithm Algoritmo de eliminação de variáveis em primeira ordem. Uma implementação do AC-FOVE é fornecida no pacote. 
Constraint Restrição do valor de uma variável lógica. O pacote possui uma implementação de restrições de desigualdade (InequalityConstraint).

Factor Fator em variáveis aleatórias parametrizadas. Existem duas implementações no pacote: StdFactor (fator genérico) e ConstantFactor (fator constante, geralmente usado com parfactors de agregação).

MacroOperation Abstração de uma macro-operação. Foram implementadas todas as macro-operações discutidas na Seção 3.3: GlobalSumOut (Eliminação Global), CountingConvert (Contagem), Propositionalize (Proposicionalização), Shatter (Particionamento), FullExpand (Expansão Total) e ConvertToStdParfactors (Conversão).

Operator Operador de agregação. São fornecidos no pacote operadores lógicos básicos: Or, And e Xor.

Parfactor Abstração com as operações comuns a todos os tipos de parfactors. É implementado nas classes StdParfactor (parfactor na forma padrão) e indiretamente por AggParfactor (parfactor de agregação).

Prv Variável aleatória parametrizada. É implementada por CountingFormula, RandomVariableSet e StdPrv.

RangeElement Elemento do domínio de uma variável aleatória parametrizada. Duas implementações são fornecidas no pacote: Bool (tipo booleano) e Histogram (histograma usado para fórmula de contagem). 


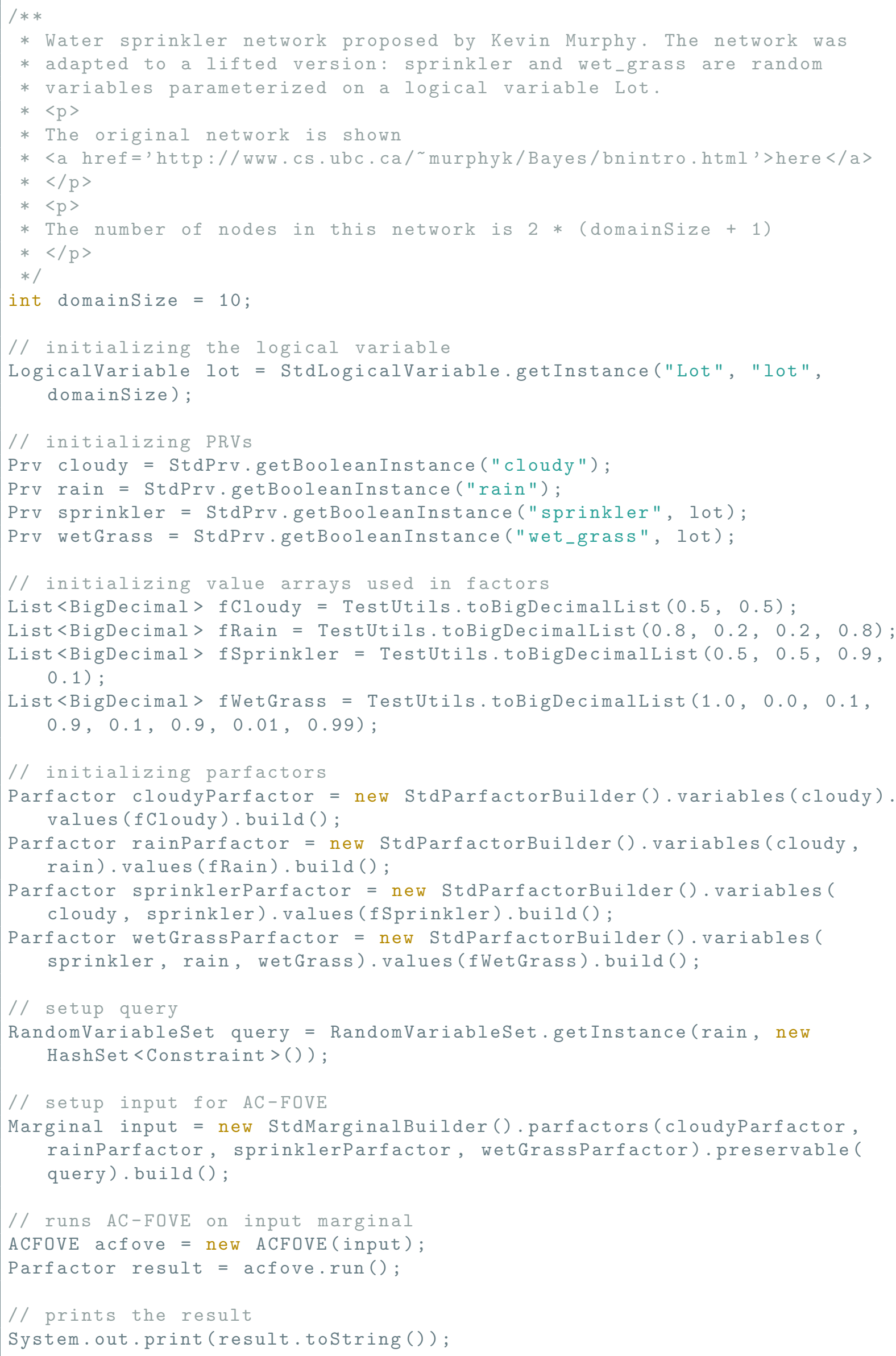

Figura 24: Código para especificação de uma rede de primeira ordem e execução do algoritmo AC-FOVE 


\section{Eliminação em parfactors de agregação}

Uma das condições impostas para a eliminação em parfactors de agregação é a inexistência da variável lógica extra nas variáveis aleatórias parametrizadas de contexto (vide Proposição 5). Esta condição não havia sido citada por Kisynski (2010) em sua formalização do algoritmo. Sem esta condição, os resultados da eliminação em parfactors de agregação se tornam incorretos, como será mostrado a seguir.

Esta condição é necessária porque a definição da sequência $\left\{\mathcal{F}_{m}\right\}$ (equação (3.2.3)) se torna inválida quando uma variável de contexto possui a variável lógica extra do nó pai da agregação. A eliminação em parfactors de agregação nada mais é que uma proposicionalização da variável lógica extra seguida de eliminações dos nós pais. Quando a variável lógica extra também está em variáveis de contexto, a proposicionalização faz com que o tamanho dos fatores $\mathcal{F}_{k}$ da sequência $\left\{\mathcal{F}_{m}\right\}$ não seja constante, com um crescimento exponencial.

Para tornar o problema mais claro, a situação é ilustrada no Exemplo 45.

Exemplo 45. Considere uma distribuição definida pelo seguinte conjunto de parfactors:

$$
\begin{aligned}
G= & \left\{\left\langle\varnothing,\{b(Y)\}, \mathcal{F}_{b}\right\rangle,\right. \\
& \left\langle\varnothing,\{b(Y), r(X, Y)\}, \mathcal{F}_{r}\right\rangle, \\
& \langle\varnothing, r(X, Y), a(X),\{b(Y)\}, \mathbb{1}, \text { o R }, Y\rangle\}
\end{aligned}
$$

Suponha que se deseja calcular

$$
\mathcal{J}_{\mathrm{RV}(a)}(G)=\sum_{b, r} \mathcal{J}(G)
$$

A variável $b(Y)$ não pode ser eliminada pois o parfactor $g_{2}$ possui 2 variáveis lógicas ( $X$ e $Y$ ). Se $X$ for eliminado de $g_{2}$ por meio de uma fórmula de contagem, posteriormente será necessário proposicionalizar $g_{3}$ para poder eliminar $\operatorname{Rv}(r(X, Y))$. 
Resta então eliminar $r(X, Y)$ usando o método proposto por Kisynski e Poole (2009). Suponha que $\mathcal{D}(Y)=\left\{y_{1}, \ldots, y_{n}\right\}$.

A cada passo do algoritmo, existe a multiplicação de pelo menos 2 fatores. Entretanto, o método proposto por Kisynski e Poole (2009) não considera quais são as variáveis aleatórias parametrizadas de cada fator, o que resulta num cálculo incorreto do marginal neste exemplo. Tome dois fatores do conjunto de fatores representado por $g_{2}$ : $\mathcal{F}\left(b\left(y_{i}\right), r\left(X, y_{i}\right)\right)$ e $\mathcal{F}\left(b\left(y_{j}\right), r\left(X, y_{j}\right)\right)$, com $i \neq j$. A multiplicação destes fatores resulta em um fator $\mathcal{F}\left(b\left(y_{i}\right), r\left(X, y_{i}\right), b\left(y_{j}\right), r\left(X, y_{j}\right)\right)$. Após a multiplicação, o método elimina uma das variáveis $r$ deste fator. Suponha, sem perda de generalidade, que o fator resultante após a eliminação é $\mathcal{F}\left(b\left(y_{i}\right), b\left(y_{j}\right), r\left(X, y_{j}\right)\right)$. Note que restam duas variáveis $b\left(y_{i}\right)$ e $b\left(y_{j}\right)$ que não podem ser condensadas em uma única variável $b(Y)$. Então, ao final do primeiro passo do algoritmo haverá um fator $\mathcal{F}_{k}$ com 3 variáveis aleatórias parametrizadas. A cada passo o fator $\mathcal{F}_{k}$ é multiplicado por outro fator idêntico, aumentando ainda mais o tamanho do fator resultante. Ao final teremos um fator com $n+1$ variáveis aleatórias parametrizadas. Se todas forem binárias, isto significa que o fator terá um tamanho $2^{n+1}$.

\subsection{Novo método de eliminação}

No Exemplo 45, foi mencionado que a variável b $(Y)$ não podia ser eliminada. Como será demonstrado a seguir, neste exemplo em particular a variável de contexto pode ser eliminada a partir de alguns ajustes na rede. Suponha que $\mathcal{D}(X)=\left\{x_{1}, \ldots, x_{m}\right\}$. A Figura 25 ilustra a rede em primeira ordem e a rede proposicionalizada representada pelos parfactors do conjunto $G$ do Exemplo 45.

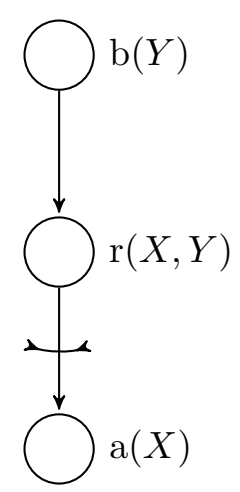

(a) Primeira ordem

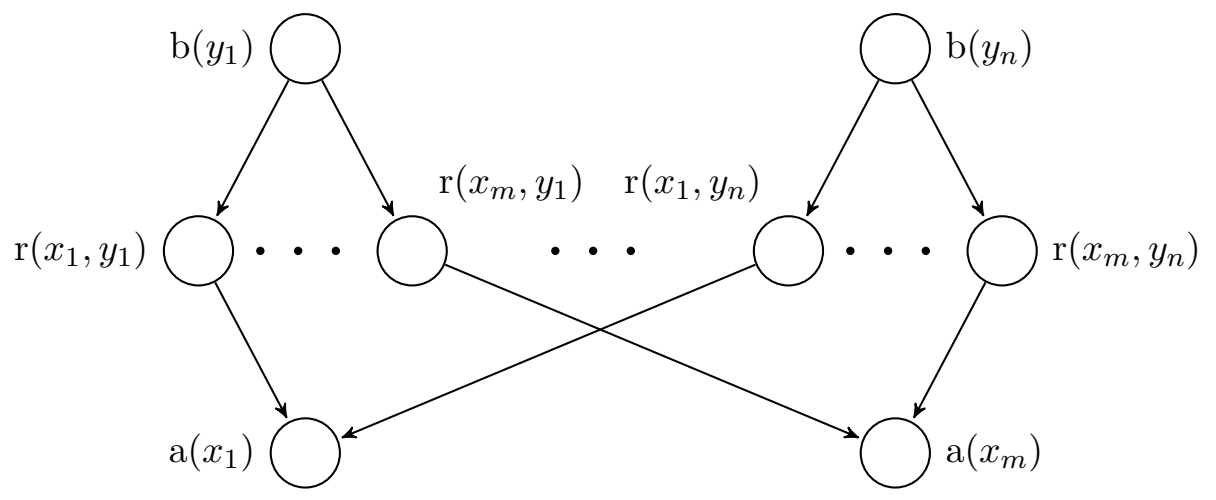

(b) Proposicionalizada

Figura 25: Representação em primeira ordem e proposicionalizada da rede do Exemplo 45 
Deseja-se calcular $\mathcal{J}_{\mathrm{Rv}(a)}(G)$, que pode ser representado por um parfactor $\langle\varnothing,\{\mathrm{a}(X)\}$, $\mathcal{F}\rangle$. Este parfactor representa $m$ fatores: $\mathcal{F}\left(a\left(x_{1}\right)\right), \ldots, \mathcal{F}\left(a\left(x_{m}\right)\right)$. Para calcular $\mathcal{F}\left(a\left(x_{1}\right)\right)$ a partir da rede proposicionalizada, pode ser feita a seguinte simplificação: todos os nós $a\left(x_{1}\right)$, $i \neq 1$ são improdutivos (isto é, não influenciam $a\left(x_{1}\right)$ ) e portanto podem ser apagados. Com isso, os nós $r\left(x_{i}, y_{j}\right), i \neq 1$ também se tornam improdutivos e podem ser ignorados. Assim, a rede simplificada é ilustrada na Figura 26.

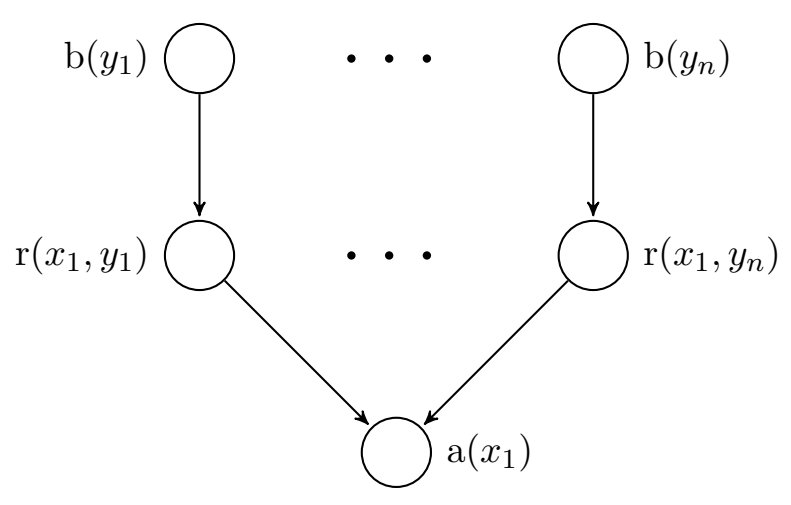

Figura 26: Representação proposicionalizada da rede do Exemplo 45, simplificada para o cálculo de $\mathcal{F}\left(a\left(x_{1}\right)\right)$

Note que o mesmo procedimento pode ser utilizado para obter os demais fatores $\mathcal{F}\left(a_{i}\right)$. Então, para qualquer $i \in\{1, \ldots, m\}$, o cálculo de $\mathcal{J}_{a\left(x_{i}\right)}(G)$ pode ser feito através da rede de primeira ordem ilustrada na Figura 27.

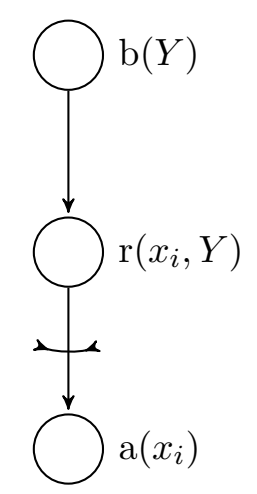

Figura 27: Representação em primeira ordem da rede da Figura 26

Os parfactors correspondentes à rede da Figura 27 são:

$$
\begin{array}{rlr}
G= & \left\{\left\langle\varnothing,\{b(Y)\}, \mathcal{F}_{b}\right\rangle,\right. & \left(g_{4}\right) \\
& \left\langle\varnothing,\left\{b(Y), r\left(x_{i}, Y\right)\right\}, \mathcal{F}_{r}\right\rangle, & \left(g_{5}\right) \\
& \left.\left\langle\varnothing, r\left(x_{i}, Y\right), a\left(x_{i}\right), \varnothing, \mathbb{1}, \text { OR }, Y\right\rangle\right\} & \left(g_{6}\right)
\end{array}
$$


Agora a variável $b(Y)$ pode ser eliminada pois o produto $g_{4} \odot g_{5}$ satisfará as condições da Proposição 2. Veja que a eliminação da variável $b(Y)$ permite a eliminação no parfactor de agregação $g_{6}$, pois não haverá mais variável de contexto contendo a variável lógica extra. Tem-se

$$
\mathcal{J}_{a\left(x_{i}\right)}(G)=\sum_{r\left(x_{i}, Y\right)} g_{6} \odot \sum_{b(Y)} g_{4} \odot g_{5}=\left\langle\varnothing,\left\{a\left(x_{i}\right)\right\}, \mathcal{F}_{i}\right\rangle
$$

Como foi mencionado anteriormente, $\mathcal{F}_{i}=\mathcal{F}$. 


\section{Experimentos}

Neste capítulo serão mostrados os experimentos realizados para comparar o desempenho de diferentes algoritmos de inferência. A métrica utilizada nos testes foi o tempo de execução do algoritmo para um dado tamanho de população. Os experimentos propostos tem o intuito de avaliar o algoritmo AC-FOVE, e não de validá-lo. A validação dos resultados produzidos pelo algoritmo foram mostrados por Kisynski (2010).

Nos experimentos foram comparados três algoritmos: eliminação de variáveis (VE), C-F Ove (MILCH et al., 2008) e A C-F Ove (KISYNSKI; POOLE, 2009). Para o A C-F Ove foi utilizada a implementação descrita no Capítulo 4. Para o C-FOVE, foi utilizada a mesma implementação do AC-F OVE após a remoção das funções relativas a parfactors de agregação. Para o VE, também foi utilizada a implementação do AC-FOVE, sendo retiradas as funções relativas a parfactors (tanto os de agregação quanto os na forma padrão) e às variáveis aleatórias parametrizadas (forma padrão e fórmulas de contagem).

Para o C-F OVE a macro-operação de conversão não está disponível, e para o algoritmo vE a única macro-operação disponível é a Eliminação Global.

Nos experimentos que se seguem, foram utilizados os mesmos modelos de primeira ordem para todos os algoritmos. Para o C-F OVE, os parfactors de agregação são convertidos para parfactors na forma padrão antes do algoritmo ser executado. De maneira análoga, para o VE o modelo de primeira ordem é transformado em um modelo de ordem zero antes da execução. Esta transformação é feita através da proposicionalização de todas as variáveis lógicas presentes na rede. O tempo gasto para transformar os parfactors não foram contabilizados no tempo de execução dos algoritmos.

Os experimentos foram executados no sistema operacional Mac OS X 10.6.8 rodando sobre processador Intel Core i5 $2.3 \mathrm{GHz}$ (dual core) e 4 GB de memória RAM (1333 MHz DDR3), dos quais 1 GB foi disponibilizado para a máquina virtual Java (JVM).

Cada ponto nos gráficos exibidos neste capítulo é a média do tempo de 10 execuções dos algoritmos nas condições dadas. Para o caso particular dos resultados do algoritmo VE, 
não foram plotados todos os pontos nos gráficos. À medida que o tamanho da população cresce, o tempo de execução do algoritmo ve cresce exponencialmente. Desse modo, estes pontos mais "avançados" não são representados pois extrapolam a área visível dos gráficos.

\subsection{Rede Sprinkler}

A rede Sprinkler foi proposta por Murphy (1998) em sua explicação introdutória sobre redes Bayesianas. A rede foi adaptada para uma versão de primeira ordem, em que os nós sprinkler $(T)$ e gramaMolhada $(T)$ são parametrizados pela variável lógica $T$ atrelada a uma população de terrenos. Para este experimento, o número de terrenos foi variado no intervalo $[1,500]$ conforme a sequência definida na Equação (6.1.1).

$$
\begin{aligned}
& n_{0}=1, \\
& n_{k}=n_{k-1}+10^{\log _{10}\left(n_{k-1}\right)} .
\end{aligned}
$$

A representação da rede em primeira ordem é apresentada na Figura 28. Os resultados do experimento são exibidos no gráfico da Figura 29.

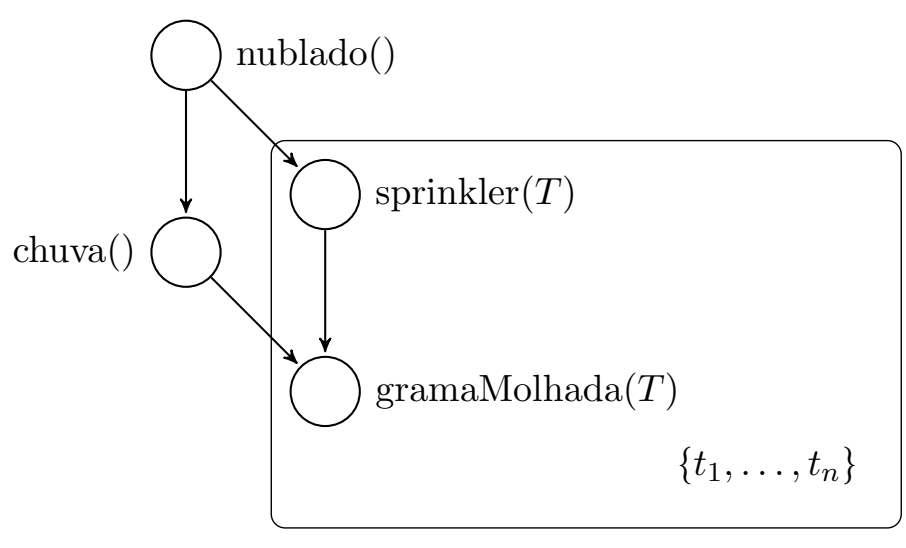

Consulta: gramaMolhada $\left(t_{1}\right)$

Figura 28: Rede Sprinkler utilizada no experimento

Os parfactors correspondentes à rede da Figura 28 são dados pelo seguinte conjunto: 


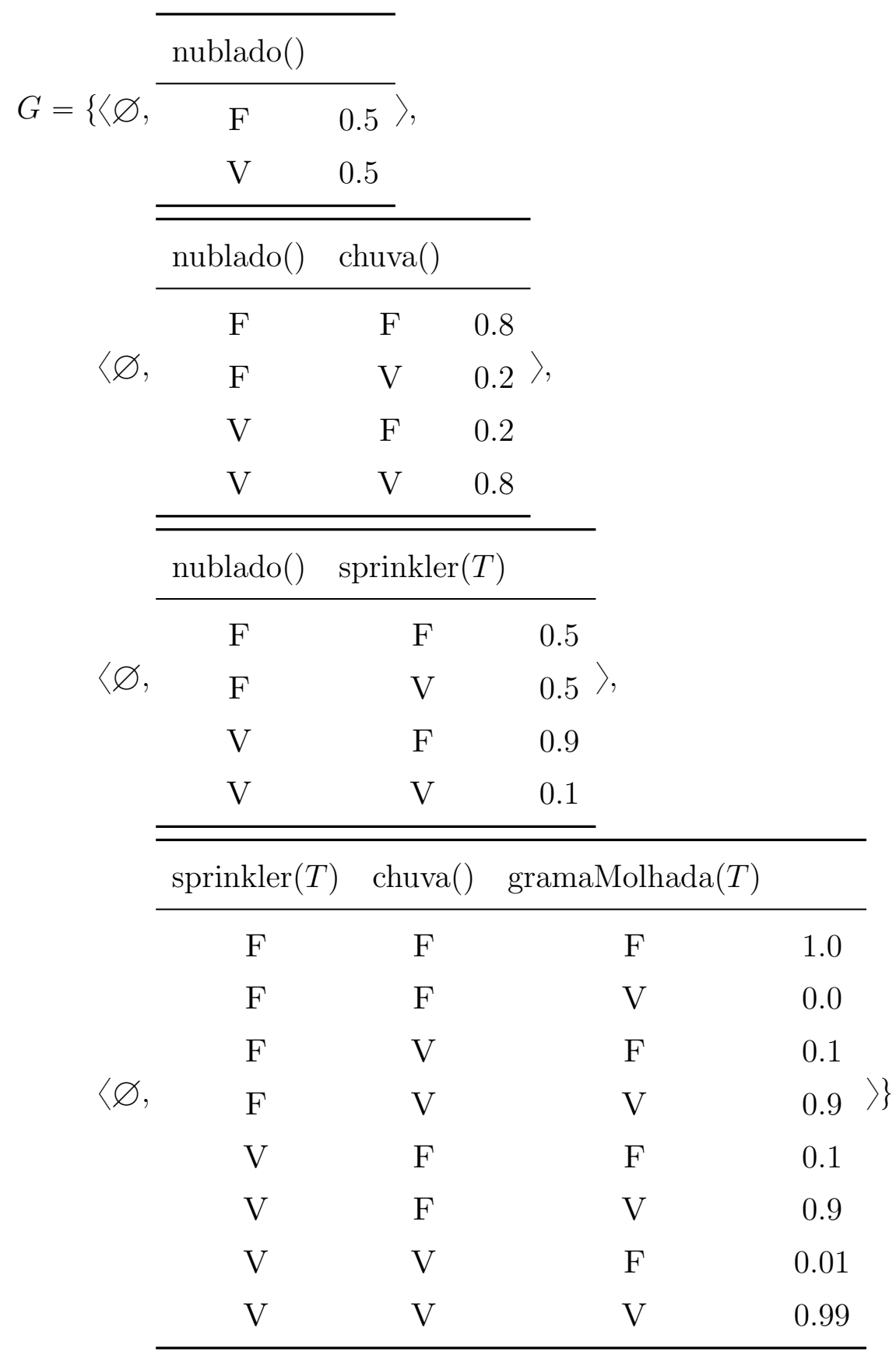




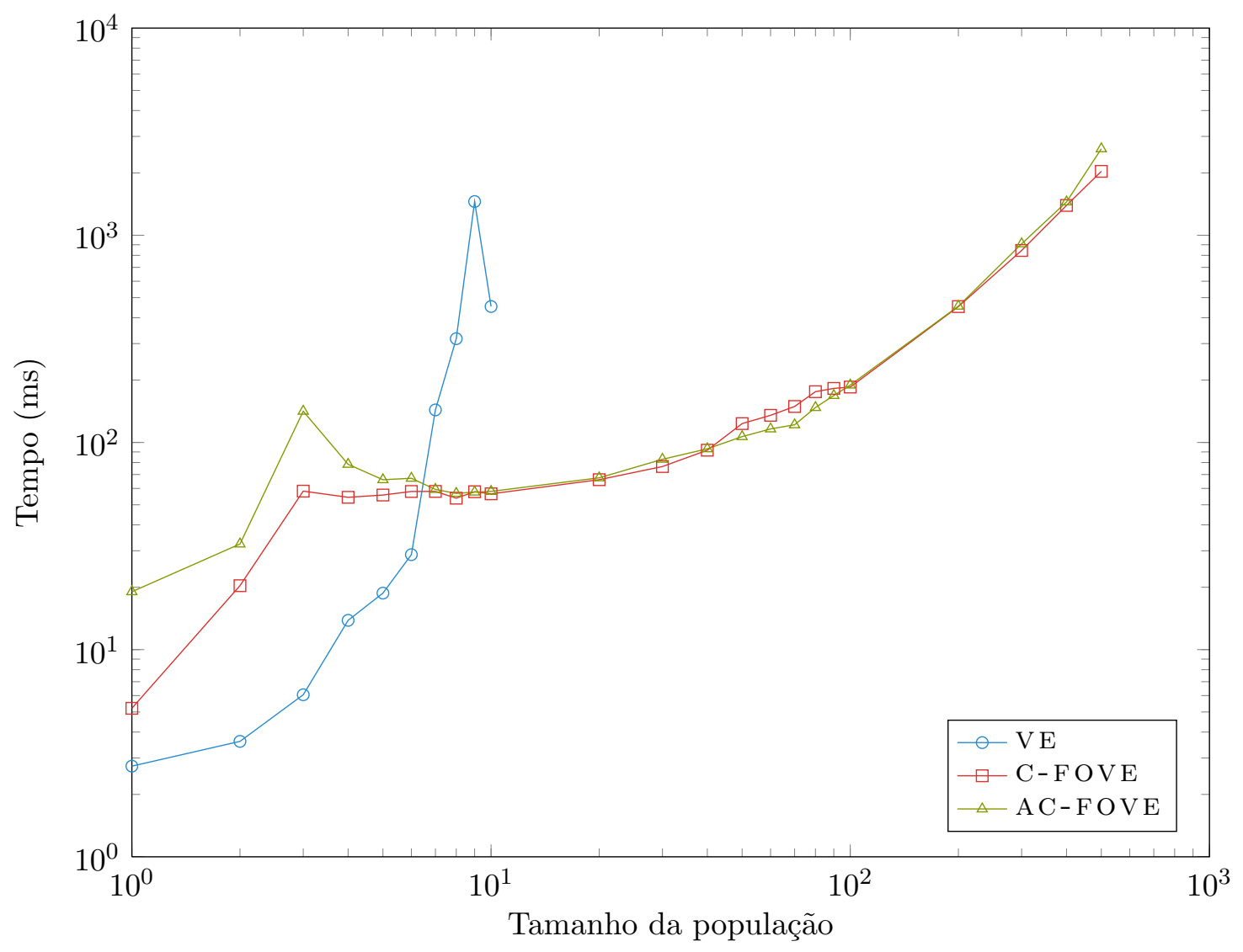

Figura 29: Média do tempo de execução dos algoritmos de inferência para a rede Sprinkler (MURPHY, 1998)

\subsection{Rede Epidemia}

A rede Epidemia foi proposta por Braz, Amir e Roth (2005) e expressa a relação entre uma epidemia e a probabilidade de morte. A rede foi adaptada para um modelo direcionado de primeira ordem (a original é uma cadeia de Markov). Além disso, foi adicionado um nó algumaMorte() para criar uma agregação. Os nós doença $(P)$ e morte $(P)$ são parametrizados pela variável lógica $P$, que está atrelada a uma população de pessoas. O número de pessoas para este experimento foi variado conforme a equação (6.1.1) no intervalo $[1,1000]$.

A representação da rede em primeira ordem é apresentada na Figura 30. Os resultados do experimento são exibidos no gráfico da Figura 31. 


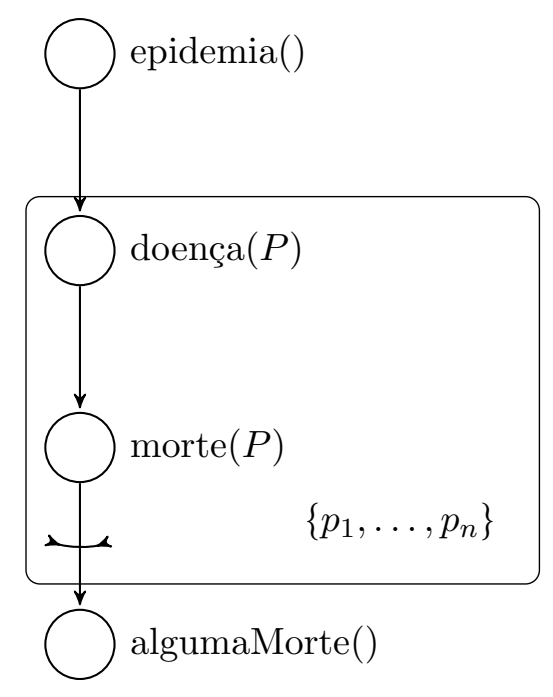

Consulta: algumaMorte()

Figura 30: Rede Epidemia utilizada no experimento

Os parfactors correspondentes à rede da Figura 30 são dados pelo seguinte conjunto:

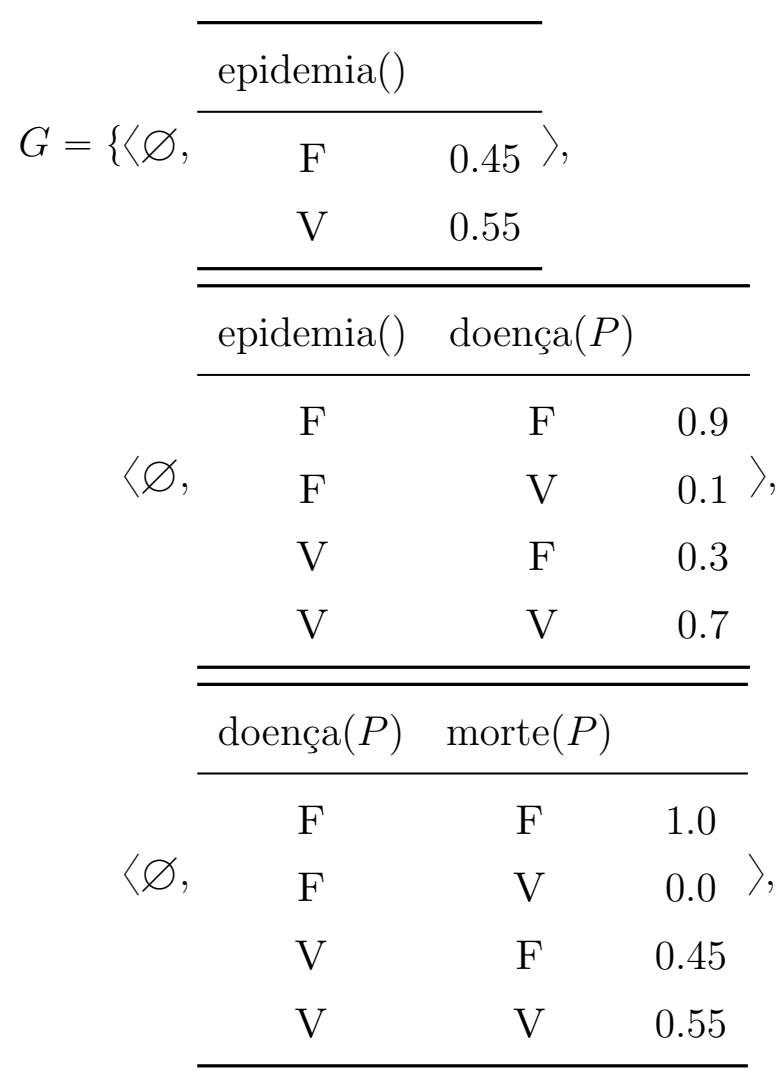

$\langle\varnothing, \operatorname{morte}(P), \operatorname{algumaMorte}(),\{\operatorname{epidemia}()\}, \mathbb{1}$, o R,$P\rangle\}$ 


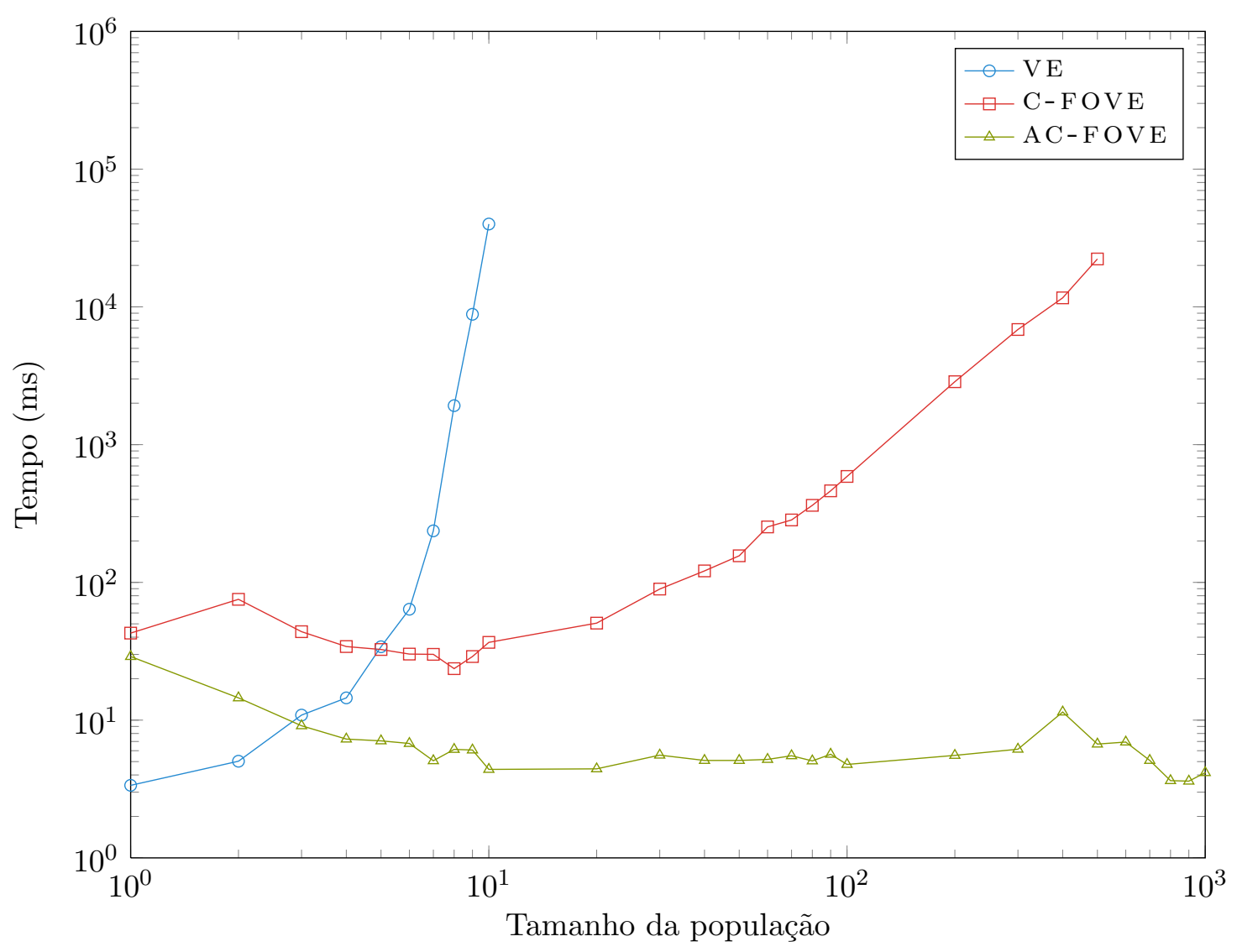

Figura 31: Média do tempo de execução dos algoritmos de inferência para a rede Epidemia (BRAZ; AMIR; ROTH, 2005)

\subsection{Rede Workshops Concorrentes}

A rede Workshops Concorrentes foi proposta por Milch et al. (2008) e modela a probabilidade de sucesso de um workshop com base no número de pessoas que comparecem, que por sua vez são influenciadas pelo tema do workshop. Esta rede foi adaptada para um modelo de primeira ordem direcionado com agregação. O nó concorrido $(W)$ é parametrizado pela variável lógica $W$, que está atrelada a uma população de workshops. O nó comparece $(P)$ é parametrizado pela variável lógica $P$, que está atrelada a uma população de pessoas.

Foram realizados dois experimentos com esta rede. No primeiro, fixou-se o número de workshops para 3 e variou-se o número de pessoas no intervalo [1,1000] utilizando a Expressão (6.1.1). No segundo experimento, fixou-se o número de pessoas para 5 e variou-se o número de workshops no intervalo [1,1000] também com base na Expressão (6.1.1). 
A representação da rede em primeira ordem é apresentada na Figura 32. Os resultados do experimento são exibidos nos gráficos das Figuras 33 e 34.

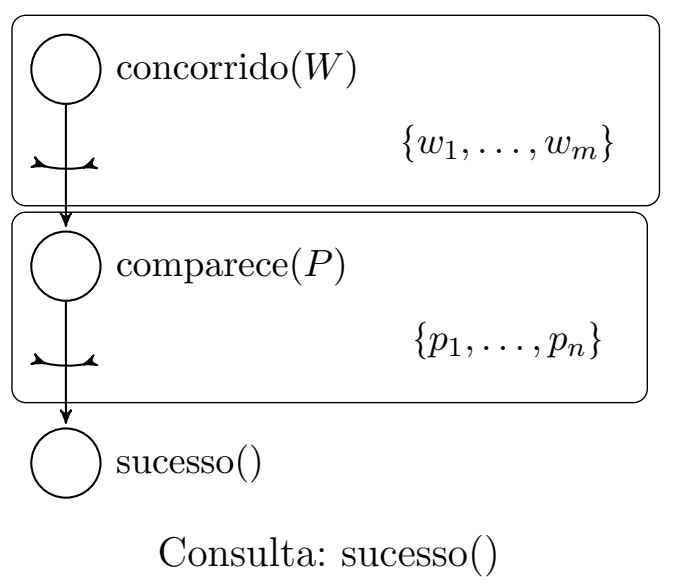

Figura 32: Rede Workshops Concorrentes utilizada no experimento

Os parfactors correspondentes à rede da Figura 32 são dados pelo seguinte conjunto:

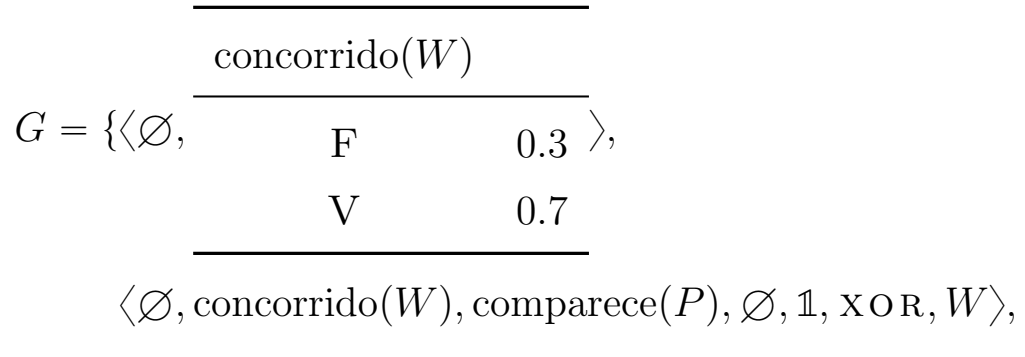

$$
\begin{aligned}
& \langle\varnothing, \operatorname{comparece}(P), \operatorname{sucesso}(), \varnothing, \mathbb{1}, \text { A N D }, P\rangle\}
\end{aligned}
$$




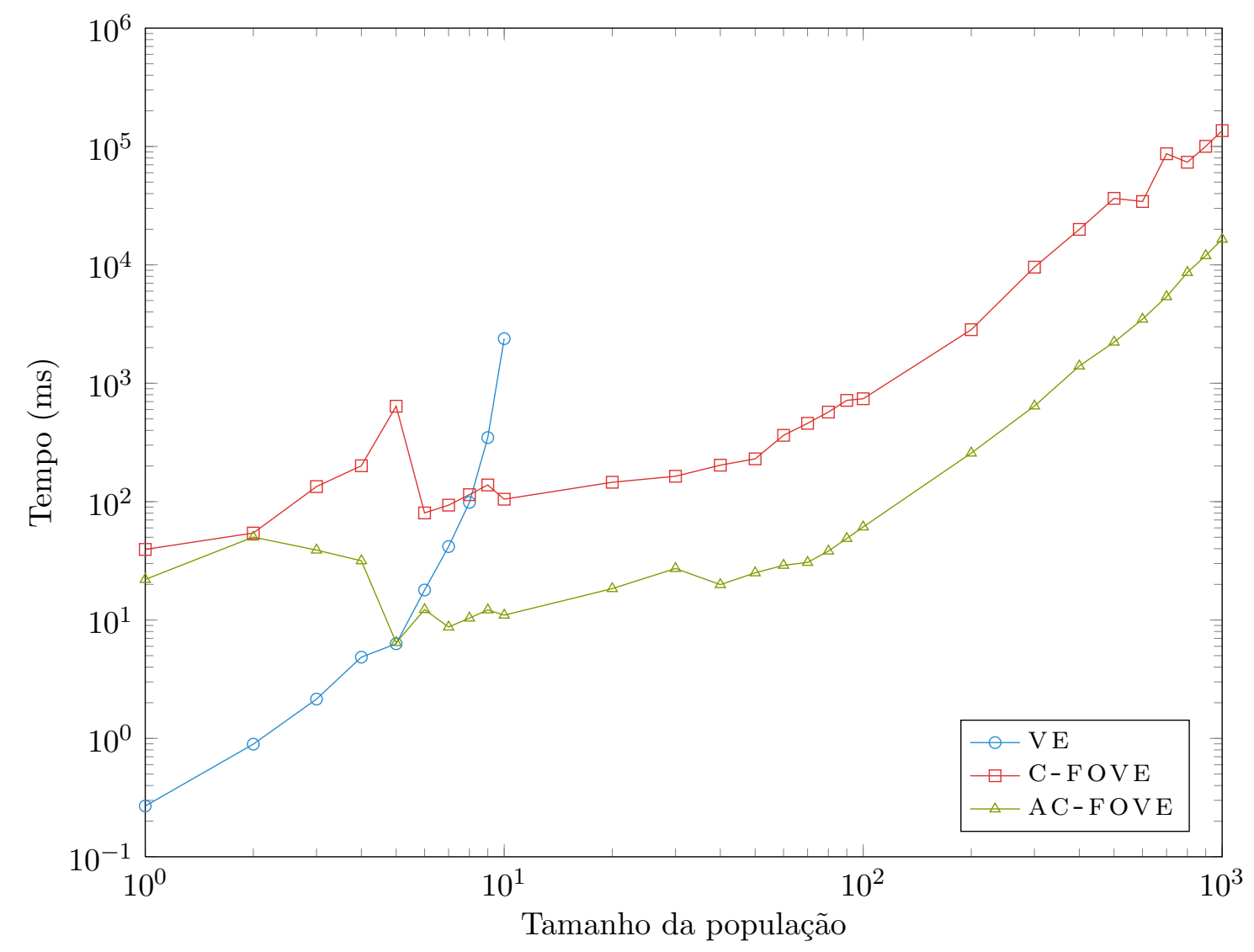

Figura 33: Média do tempo de execução dos algoritmos de inferência para a rede Workshops Concorrentes (MILCH et al., 2008) com 3 workshops 


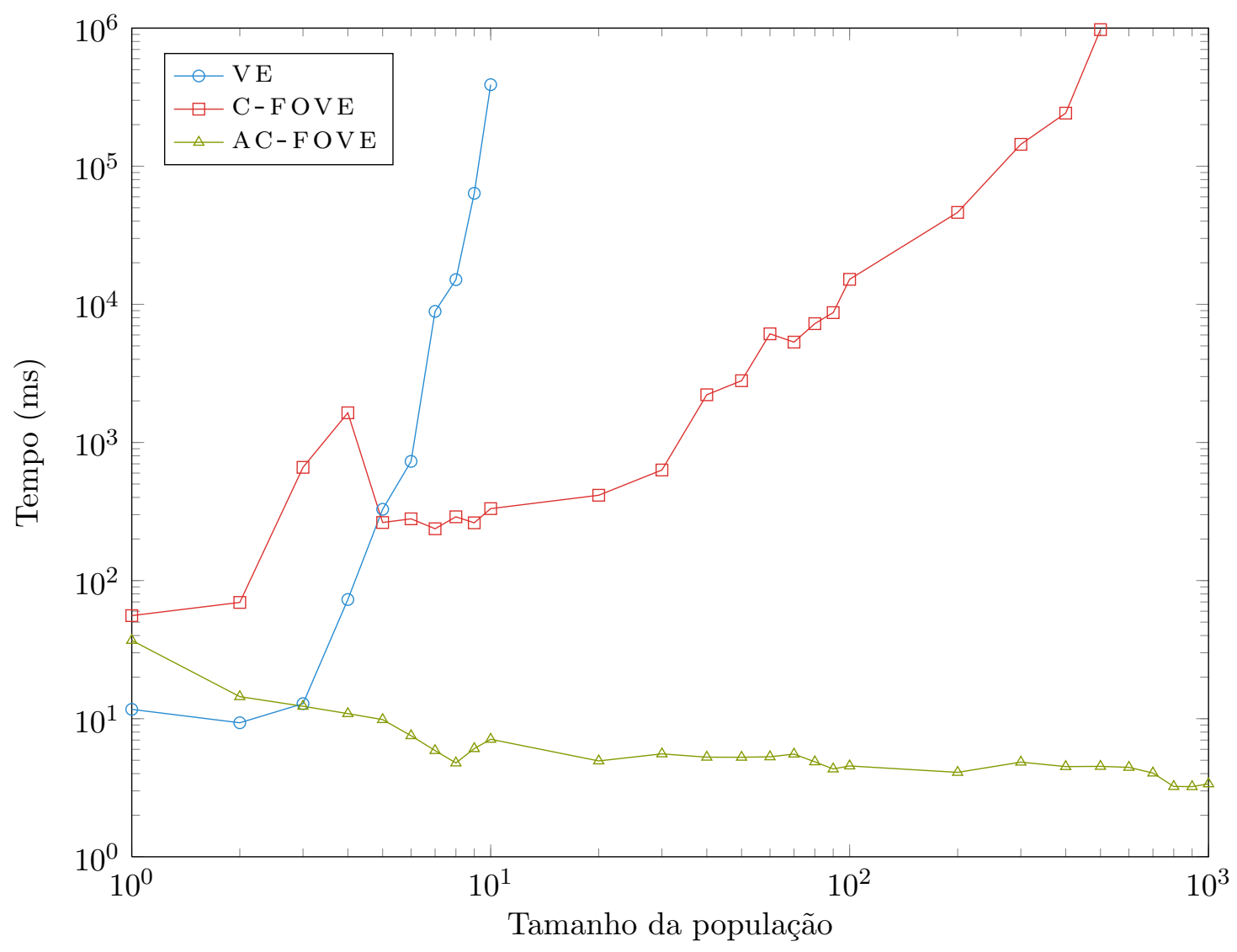

Figura 34: Média do tempo de execução dos algoritmos de inferência para a rede Workshops Concorrentes (MILCH et al., 2008) com 5 pessoas

\subsection{Rede Loteria}

A rede Loteria foi proposta por Kisynski (2010) e representa os fatores que influenciam na probabilidade de ganhar na loteria. Este fato é afetado pelo número de pessoas que jogam e acertam todos os números e pelo prêmio que será pago ao ganhador (quanto maior o prêmio, maior a probabilidade de uma pessoa apostar). Os nós jogou $(P)$ e acertou6 $(P)$ são parametrizados pela variável lógica $P$ que está atrelada a uma população de pessoas. $\mathrm{O}$ número de pessoas no experimento foi variado no intervalo $[1,1000]$ conforme a expressão (6.1.1).

Esta rede já foi utilizada no Exemplo 44 e será reproduzida a seguir para uma referência mais fácil. A representação da rede em primeira ordem é apresentada na Figura 35. Os resultados do experimento são exibidos no gráfico da Figura 36. 


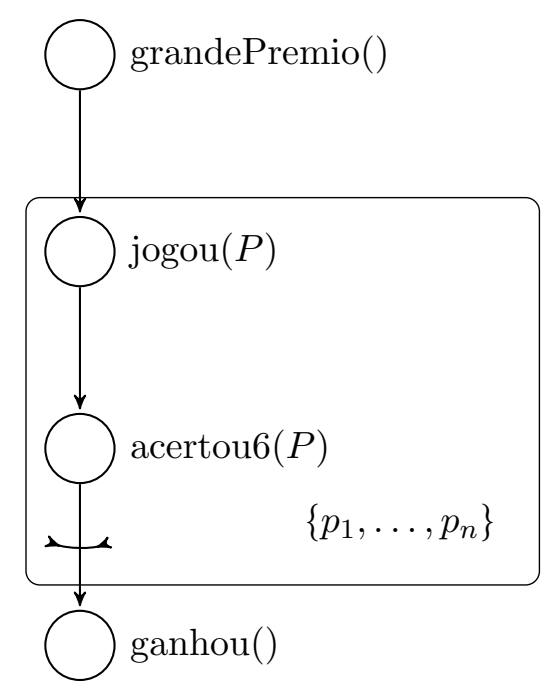

Consulta: ganhou()

Figura 35: Rede Loteria utilizada no experimento

Os parfactors correspondentes à rede da Figura 35 são dados pelo seguinte conjunto:

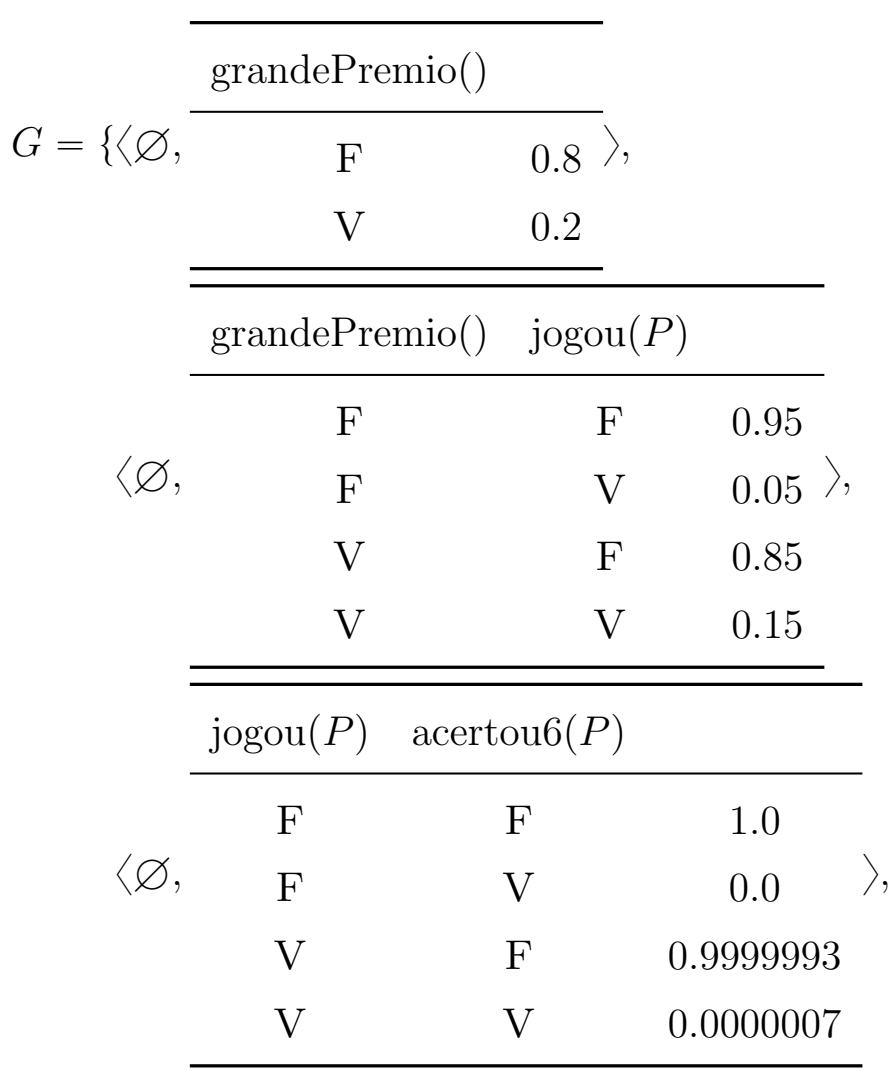

$\langle\varnothing, \operatorname{acertou} 6(P), \operatorname{ganhou}(),\{\operatorname{grandePremio}()\}, \mathbb{1}$, o R,$P\rangle\}$ 


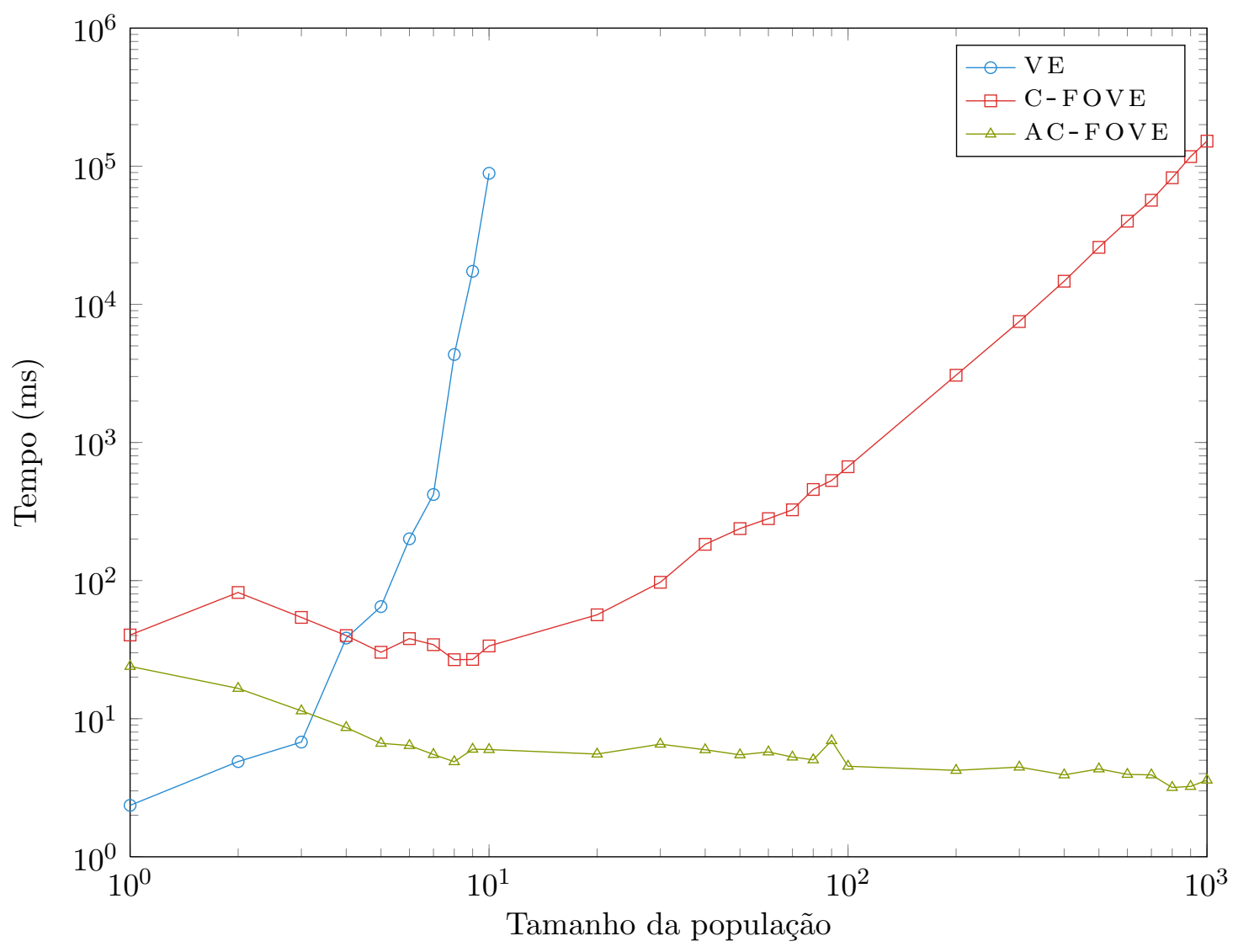

Figura 36: Média do tempo de execução dos algoritmos de inferência para a rede Loteria (KISYNSKI, 2010)

\subsection{Análise dos Resultados}

Em todos os experimentos realizados nota-se que o tempo de execução do algoritmo VE possui uma tendência de crescimento muito acima dos demais algoritmos. Pode ser provado (KISYNSKI, 2010) que a complexidade de tempo e de espaço do algoritmo VE é exponencial no tamanho da população. Como não foi aplicada nenhuma heurística de ordenação para a eliminação, o tempo de inferência se torna alto (maior que $10^{6} \mathrm{~ms}$ ) mesmo para populações com menos de 10 indivíduos e modelos extremamente simples.

Na rede Sprinkler, os algoritmos C-FOVE e AC-F OVE tiveram desempenhos semelhantes. Pode-se afirmar que ambos os algoritmos possuem o mesmo comportamento neste problema, pois não existe agregação no modelo e portanto o AC-FOVE não possuirá nenhum diferencial com relação ao C-F OVE.

Na rede Epidemia, nota-se que, enquanto o algoritmo C-FOVE possui um tempo de inferência que depende do tamanho da população, o algoritmo A C-F OVE pôde computar 
o resultado em tempo praticamente constante devido ao uso das operações de agregação.

No experimento com a rede Workshops Concorrentes, observou-se comportamentos distintos nas duas situações apresentadas. Na primeira situação, em que o número de workshops é fixo, os algoritmos AC-FOVE e C-FOVE possuem a mesma tendência de crescimento. Já na segunda situação, em que o número de pessoas é fixo, o tempo de inferência pelo algoritmo A C-F OVE se mantém praticamente constante. Isto ocorre porque durante a inferência é criada uma fórmula de contagem na variável lógica atrelada à população de pessoas. Quando o número de pessoas é fixo, o tempo de inferência se torna independente do tamanho das populações.

Na rede Loteria, observa-se um comportamento semelhante ao da rede Epidemia. De fato, os dois problemas são praticamente idênticos.

Em suma, o algoritmo A C-F OVE possui um desempenho igual ou superior ao C-F OVE quando comparados em relação ao tempo de execução de inferência. Isto já era um resultado esperado na teoria, uma vez que o AC-FOVE é um C-FOVE com otimizações. Ambos os algoritmos apresentam desempenho muito superior ao algoritmo $\mathrm{VE}$ quando aplicados a modelos de primeira ordem. 


\section{Considerações finais}

Neste trabalho, foi desenvolvida uma implementação do algoritmo AC-F OVE para inferências em modelos de primeira ordem, cuja inspiração vem do procedimento de eliminação de variáveis em redes Bayesianas. A teoria envolvida na formulação do algoritmo foi detalhada e elucidada com exemplos, além de terem sido formulados complementos considerados faltantes na sua concepção original:

- Fórmulas para o cálculo de custos do algoritmo;

- Correção da proposição 3.6 de Kisynski (2010), que trata da eliminação em parfactors de agregação.

Inicialmente, a proposta do trabalho era utilizar o algoritmo A C-F OVE para realizar inferências na lógica de descrição probabilística CR $\mathcal{A L C}$ (TAKIYAMA; COZMAN, 2012b, 2012a; POLASTRO et al., 2012). Entretanto, devido às limitações do algoritmo, decidiuse implementá-lo em sua forma genérica, isto é, de maneira independente da linguagem utilizada para descrever os modelos de primeira ordem.

O objetivo foi alcançado por meio do estudo do algoritmo proposto por Kisynski e Poole (2009), bem como de trabalhos anteriores (MILCH et al., 2008; BRAZ; AMIR; ROTH, 2007). Após o estudo inicial, utilizou-se uma metodologia ágil para codificar o software que implementa o algoritmo. Durante a implementação, percebeu-se a falta de alguns detalhes não formalizados no algoritmo, o que resultou também em uma contribuição teórica.

Os experimentos realizados mostram que a implementação proposta é capaz de lidar com agregação de maneira eficiente se comparado com a versão anterior do A C-F OVE, o C-F O vE (MILCH et al., 2008). Em alguns dos modelos mostrados, o tempo de inferência permaneceu praticamente constante (independente do número de nós do modelo), enquanto que os algoritmos C-FOVE e VE apresentaram um tempo de inferência dependente do tamanho do modelo. Evidentemente, os modelos utilizados nos experimentos não têm a complexidade dos modelos que podem ser encontrados no mundo real, no entanto são 
válidos para os testes iniciais de qualquer algoritmo de inferência em primeira ordem: se não forem capazes de lidar com estes modelos menores, provavelmente não serão utilizáveis para modelos mais complexos.

Este trabalho apresentou como principais contribuições:

- A implementação do algoritmo AC-F OVE em um pacote de software, juntamente com a sua documentação;

- A proposta de fórmulas analíticas para o cálculo do custo de macro-operações;

- A proposta de uma nova condição para eliminação em parfactors de agregação, sem a qual os resultados se tornam incorretos;

- A proposta de uma solução para o problema que o A C-F OVE não pôde resolver.

O último item das contribuições abre caminhos para novas possibilidades de inferência em primeira ordem. No caso, foi utilizada uma técnica já utilizada para otimizar inferências em redes Bayesianas (poda). A investigação da viabilidade de aplicação de tais técnicas em modelos de primeira ordem é um tema interessante para trabalhos futuros. Outra possibilidade é a investigação de ampliação da expressividade dos parfactors de maneira a realizar consultas mais flexíveis, como por exemplo permitir o uso de outros tipos de restrição além da desigualdade. Este aumento de expressividade pode aumentar também o tempo para o processamento das restrições durante a fase de particionamento, e analisar o ganho obtido (se ele existir) é um desafio interessante.

Outras extensões que poderiam ser estudadas são: a computação de inferências para domínios infinitos, o desenvolvimento de módulos para aplicação do algoritmo a linguagens específicas, como a CR $\mathcal{A L C}$ (POLASTRO, 2012) e o desenvolvimento de uma interface gráfica e/ou textual para a implementação corrente do algoritmo. 


\section{Referências}

BRAZ, R. de S.; AMIR, E.; ROTH, D. Lifted first-order probabilistic inference. In: KAELBLING, L. P.; SAFFIOTTI, A. (Ed.). 19th International Joint Conference on Artificial Intelligence. Edinburgh, Scotland, UK: Professional Book Center, 2005. (IJCAI'05), p. 1319-1125.

BRAZ, R. de S.; AMIR, E.; ROTH, D. MPE and partial inversion in lifted probabilistic variable elimination. In: COHN, A. (Ed.). 21st National Conference on Artificial Intelligence. Boston, Massachusetts: AAAI Press, 2006. (AAAI'06, v. 2), p. 1124-1130.

BRAZ, R. de S.; AMIR, E.; ROTH, D. Lifted first-oder probabilistic inference. In: GETOOR, L.; TASKAR, B. (Ed.). Introduction to Statistical Relational Learning. Cambridge: MIT Press, 2007. cap. 15, p. 433-450.

BREESE, J. S. Construction of belief and decision networks. Computational Intelligence, v. 8, p. $624-647,1992$.

BUNTINE, W. L. Operations for learning with graphical models. Journal of Artificial Intelligence Research, v. 2, p. 159-225, 1994.

CHARNIAK, E. Bayesian networks without tears. AI Magazine, p. 50-63, 1991.

DZHUROV, Y.; KRASTEVA, I.; ILIEVA, S. Personal extreme programming - an agile process for autonomous developers. In: Proceedings International Conference Software, Services 85 Semantic Technologies. Sofia, Bulgaria: Demetra EOOD, 2009. (ST3'09).

FELLER, W. An Introduction to Probability Theory and its Applications. New York: J. Wiley \& Sons, 1968.

GETOOR, L.; TASKAR, B. Introduction to Statistical Relational Learning (Adaptive Computation and Machine Learning). Boston, Massachusetts: The MIT Press, 2007.

HORSCH, M. C.; POOLE, D. A dynamic approach to probabilistic inference using Bayesian networks. In: BONISSONE, P. et al. (Ed.). 6th Conference on Uncertainty in Artificial Intelligence. Corvallis, Oregon, USA: UAI Press, 1990. (UAI'90), p. 155-161.

JANZEN, D.; SAIEDIAN, H. Test-driven development: Concepts, taxonomy, and future direction. Computer, IEEE Computer Society Press, v. 38, n. 9, p. 43-50, 2005.

KISYNSKI, J. Aggregation and Constraint Processing in Lifted Probabilistic Inference. Tese (Doutorado) - University of British Columbia, 2010.

KISYNSKI, J.; POOLE, D. Lifted aggregation in directed first-order probabilistic models. In: KITANO, H. (Ed.). 21st International Joint Conference on Artifical Intelligence. Burlington, Massachusetts, USA: Morgan Kaufmann Publishers Inc., 2009. (IJCAI'09), p. 1922-1929. 
MACKWORTH, A. Consistency in networks of relations. Artificial Intelligence, v. 8, n. 1, p. 99-118, 1977.

MILCH, B. et al. Lifted probabilistic inference with counting formulas. In: COHN, A. (Ed.). 23rd National Conference on Artificial Intelligence. Boston, Massachusetts, USA: AAAI Press, 2008. (AAAI'08, v. 2), p. 1062-1068.

MURPHY, K. A Brief Introduction to Graphical Models and Bayesian Networks. 1998. Acessado em: 8 out. 2013. Disponível em: <http://www.cs.ubc.ca/ murphyk/Bayes/ bnintro.html>.

PEARL, J. Probabilistic Reasoning in Intelligent Systems: Networks of Plausible Inference. San Francisco, CA, USA: Morgan Kaufmann Publishers Inc., 1988.

POLASTRO, R. B. Lógica Probabilística Baseada em Redes Bayesianas Relacionais com Inferência em Primeira Ordem. Tese (Doutorado) - Escola Politécnica da Universidade de São Paulo, 2012.

POLASTRO, R. B.; COZMAN, F. G. Loopy propagation in a probabilistic description logic. In: 2nd International Conference on Scalable Uncertainty Management. Berlin, Germany: Springer-Verlag, 2008. p. 120-133.

POLASTRO, R. B. et al. Computing inferences for credal alc terminologies. In: 8th International Workshop on Uncertainty Reasoning for the Semantic Web. [S.1.: s.n.], 2012. p. $94-97$.

POOLE, D. First-order probabilistic inference. In: 18th International Joint Conference on Artificial Intelligence. San Francisco, CA, USA: Morgan Kaufmann Publishers Inc., 2003. (IJCAI'03), p. 985-991.

POOLE, D.; MACKWORTH, A. Artificial Intelligence: Foundations of Computational Agents. USA: Cambridge University Press, 2010.

RAEDT, L. D. et al. (Ed.). Probabilistic Inductive Logic Programming - Theory and Applications, v. 4911 de Lecture Notes in Computer Science, (Lecture Notes in Computer Science, v. 4911). Berlin, Germany: Springer, 2008.

RUSSELL, S. J.; NORVIG, P. Artificial Intelligence: A Modern Approach. Upper Saddle River, NJ, USA: Prentice-Hall, Inc., 1995.

SCHMIDT-SCHAUSS, M.; SMOLKA, G. Attributive concept descriptions with complements. Artificial Intelligence, v. 48, p. 1-26, 1991.

STERLING, L.; SHAPIRO, E. The Art of Prolog: Advanced Programming Techniques. 2. ed. Cambridge, MA, USA: MIT Press, 1994.

TAKIYAMA, F. I.; COZMAN, F. G. Algoritmo de eliminação de variáveis em primeira ordem aplicado a uma lógica de descrição probabilística. In: IX Encontro Nacional de Inteligência Artificial. Curitiba, Brasil: [s.n.], 2012. p. 1-12.

TAKIYAMA, F. I.; COZMAN, F. G. Inferences on cralc using the ac-fove algorithm. In: XIX Congresso Brasileiro de Automática. Campina Grande, Brasil: [s.n.], 2012. p. $1857-1862$. 
YEDIDIA, J. S.; FREEMAN, W. T.; WEISS, Y. Constructing free energy approximations and generalized belief propagation algorithms. IEEE Transactions on Information Theory, v. 51, p. 2282-2312, 2005.

ZHANG, N. L.; POOLE, D. A simple approach to Bayesian network computations. In: 10th Biennial Canadian Artificial Intelligence Conference. [S.l.: s.n.], 1994. (AI'1994), p. $171-178$.

ZHANG, N. L.; POOLE, D. Exploiting causal independence in Bayesian network inference. Journal of Artificial Intelligence Research, v. 5, p. 301-328, 1996. 


\section{APÊNDICE A - Credal ALC e Parfactors}

$\mathrm{CR} \mathcal{A L C}$ é uma lógica de descrição probabilística proposta por Polastro e Cozman (2008) que combina estruturas da lógica $\mathcal{A L C}$ com asserções probabilísticas. As terminologias expressas em $\mathrm{CR} \mathcal{A} \mathcal{L C}$ podem ser representadas através de Redes Bayesianas Relacionais (RBN) pois a condição de Markov é satisfeita (POLASTRO; COZMAN, 2008).

Tradicionalmente, cada nó de uma Rede Bayesiana representa uma variável aleatória. Em Redes Bayesianas Relacionais, as variáveis aleatórias (e os respectivos nós) são parametrizadas; cada parâmetro está associado a uma população e a substituição dos parâmetros por indivíduos da população em uma variável aleatória parametrizada gera uma variável aleatória. Desse modo, uma variável aleatória parametrizada representa um conjunto de variáveis aleatórias, uma para cada substituição possível dos parâmetros pelos indivíduos da população. O conceito de variável aleatória parametrizada é formalizado na Seção 3.1.1.

Nesta seção a representação de sentenças da CR $\mathcal{A L C}$ em Redes Bayesianas Relacionais é detalhada. Para que isto seja possível, assumem-se as seguintes premissas:

-Nesta seção em particular, para manter consistência com a notação utilizada por Polastro e Cozman (2008), as variáveis lógicas serão representadas por letras minúsculas;

-O conceito $C(x)$ é uma variável aleatória booleana parametrizada em $x$, de modo que $C(a), a \in \mathcal{D}(x)$ é uma variável aleatória; assim, Mãe(Maria) $=V$ indica que o indivíduo Maria é Mãe;

-O papel $r(x, y)$ é uma variável aleatória booleana parametrizada em $x$ e $y$, de modo que $r(a, b), a \in \mathcal{D}(x)$ é uma variável aleatória. Assim, éIrmão(Maria, João) $=V$ indica que o indivíduo Maria é irmã do indivíduo João.

Cada nó da rede possuirá um fator, que representa a distribuição de probabilidade associada a ele. No resto desta seção, assume-se que $C(x), A(x)$, e $B(x)$ são conceitos e $r(x, y)$ é um papel. Para cada estrutura da lógica $\mathrm{CR} \mathcal{A L C}$, apresenta-se a Rede Bayesiana 
Relacional correspondente e a Rede Bayesiana resultante de sua proposicionalização. Para manter a simplicidade dos exemplos apresentados, omitem-se os fatores dos nós irrelevantes e assume-se $\mathcal{D}(x)=\left\{x_{0}, x_{1}\right\}$ e $\mathcal{D}(y)=\left\{y_{0}, y_{1}\right\}$.

\section{A.1 Atribuições de probabilidade}

Atribuições de probabilidade são diretamente representáveis em RBN. Dadas as expressões $P(A)=\alpha_{1}, P(B \mid A)=\alpha_{2}$ e $P(r)=\alpha_{3}$, a Figura 37 apresenta a rede correspondente. Note que o fator do nó $B(x)$ não está completamente especificado; para isto seria necessário explicitar o valor de $P(\neg B \mid A)$ ou de $P(\neg B \mid \neg A)$.

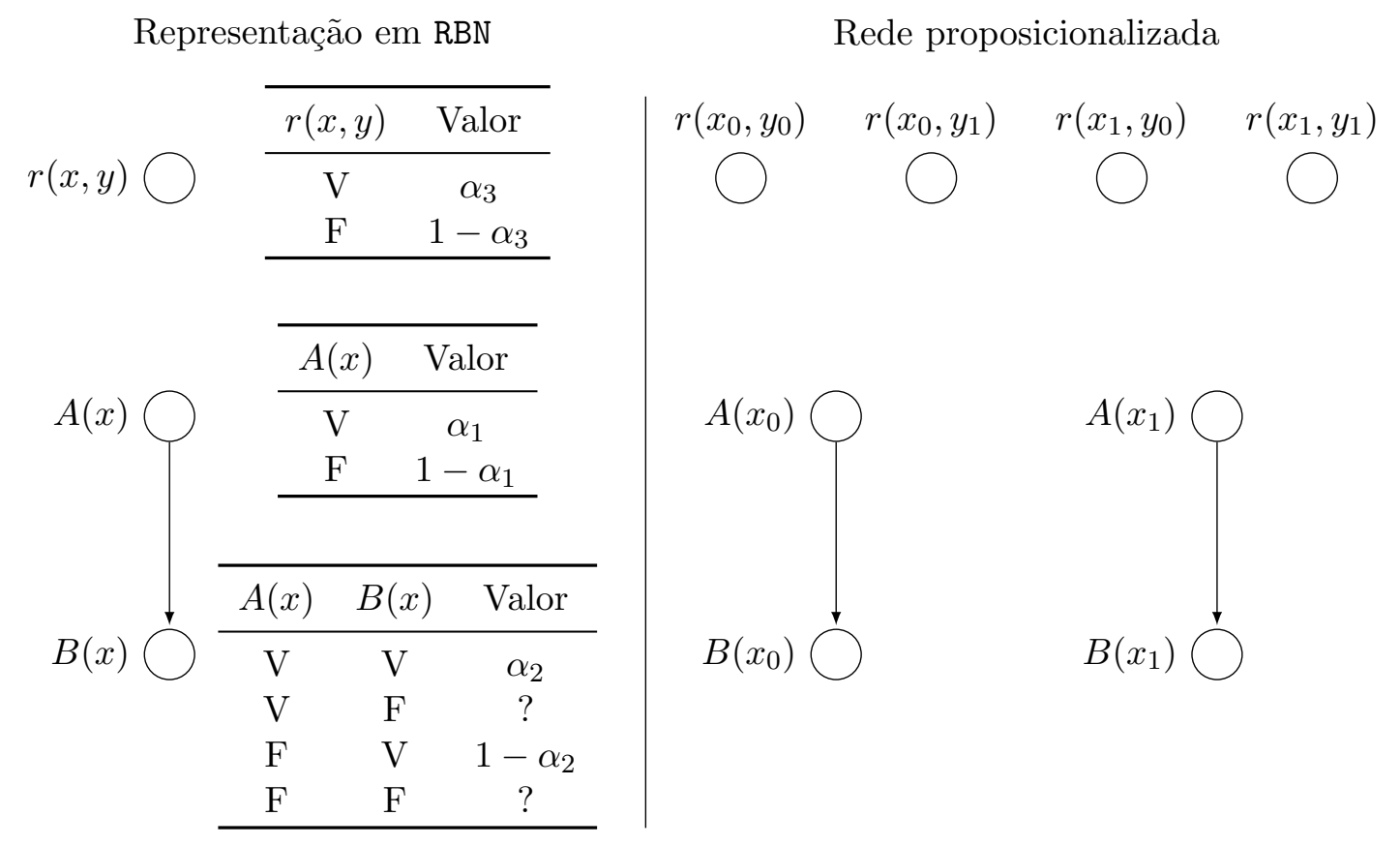

Figura 37: Representação das atribuições $P(A)=\alpha_{1}, P(B \mid A)=\alpha_{2}$ e $P(r)=\alpha_{3}$.

\section{A.2 Definição e inclusão de conceitos}

A expressão $A \equiv B$ é equivalente a $P(A \mid B)=1$ e $P(A \mid \neg B)=0$. A expressão $A \sqsubseteq B$ é equivalente a $P(A \mid \neg B)=0$. A Figura 38 ilustra a representação de $A \equiv B$ e a Figura 39 ilustra a representação de $A \sqsubseteq B$. Note que a inclusão $A \sqsubseteq B$ não é suficiente para especificar completamente o fator do nó $B$. 
Representação em RBN

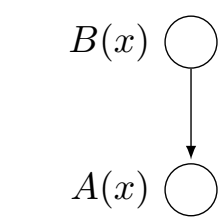

\begin{tabular}{ccc}
\hline$A(x)$ & $B(x)$ & Valor \\
\hline $\mathrm{V}$ & $\mathrm{V}$ & 1 \\
$\mathrm{~V}$ & $\mathrm{~F}$ & 0 \\
$\mathrm{~F}$ & $\mathrm{~V}$ & 0 \\
$\mathrm{~F}$ & $\mathrm{~F}$ & 1 \\
\hline
\end{tabular}

Rede proposicionalizada
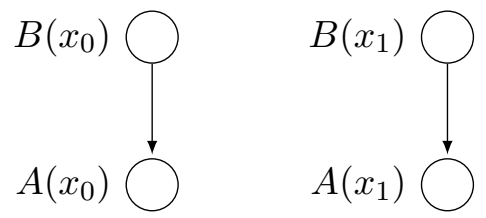

Figura 38: Representação da definição $A \equiv B$.

Representação em RBN

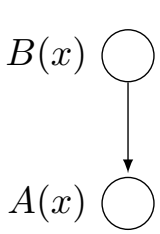

\begin{tabular}{ccc}
\hline$A(x)$ & $B(x)$ & Valor \\
\hline $\mathrm{V}$ & $\mathrm{V}$ & $?$ \\
$\mathrm{~V}$ & $\mathrm{~F}$ & 0 \\
$\mathrm{~F}$ & $\mathrm{~V}$ & $?$ \\
$\mathrm{~F}$ & $\mathrm{~F}$ & 1 \\
\hline
\end{tabular}

Rede proposicionalizada

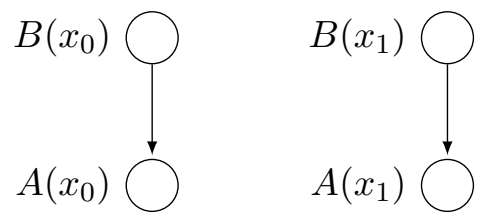

Figura 39: Representação da inclusão $A \sqsubseteq B$.

\section{A.3 Construtores lógicos}

As Figuras 40, 41 e 42 ilustram as representações das expressões $C \equiv A \sqcup B, C \equiv A \sqcap B$ e $C \equiv \neg A$, respectivamente. 
Representação em RBN

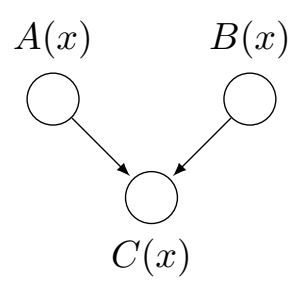

\begin{tabular}{cccc}
\hline$A(x)$ & $B(x)$ & $C(x)$ & Valor \\
\hline $\mathrm{V}$ & $\mathrm{V}$ & $\mathrm{V}$ & 1 \\
$\mathrm{~V}$ & $\mathrm{~V}$ & $\mathrm{~F}$ & 0 \\
$\mathrm{~V}$ & $\mathrm{~F}$ & $\mathrm{~V}$ & 1 \\
$\mathrm{~V}$ & $\mathrm{~F}$ & $\mathrm{~F}$ & 0 \\
$\mathrm{~F}$ & $\mathrm{~V}$ & $\mathrm{~V}$ & 1 \\
$\mathrm{~F}$ & $\mathrm{~V}$ & $\mathrm{~F}$ & 0 \\
$\mathrm{~F}$ & $\mathrm{~F}$ & $\mathrm{~V}$ & 0 \\
$\mathrm{~F}$ & $\mathrm{~F}$ & $\mathrm{~F}$ & 1 \\
\hline
\end{tabular}

Rede proposicionalizada
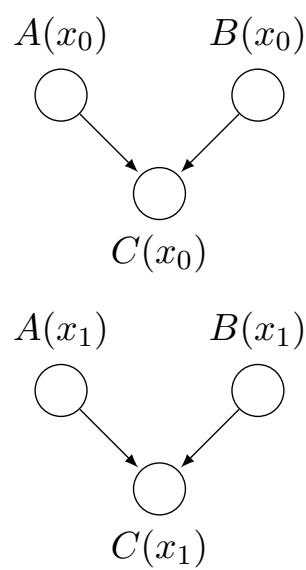

Figura 40: Representação da disjunção $C \equiv A \sqcup B$.

Representação em RBN

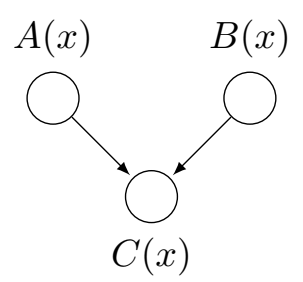

\begin{tabular}{cccc}
\hline$A(x)$ & $B(x)$ & $C(x)$ & Valor \\
\hline $\mathrm{V}$ & $\mathrm{V}$ & $\mathrm{V}$ & 1 \\
$\mathrm{~V}$ & $\mathrm{~V}$ & $\mathrm{~F}$ & 0 \\
$\mathrm{~V}$ & $\mathrm{~F}$ & $\mathrm{~V}$ & 0 \\
$\mathrm{~V}$ & $\mathrm{~F}$ & $\mathrm{~F}$ & 1 \\
$\mathrm{~F}$ & $\mathrm{~V}$ & $\mathrm{~V}$ & 0 \\
$\mathrm{~F}$ & $\mathrm{~V}$ & $\mathrm{~F}$ & 1 \\
$\mathrm{~F}$ & $\mathrm{~F}$ & $\mathrm{~V}$ & 0 \\
$\mathrm{~F}$ & $\mathrm{~F}$ & $\mathrm{~F}$ & 1 \\
\hline
\end{tabular}

Rede proposicionalizada
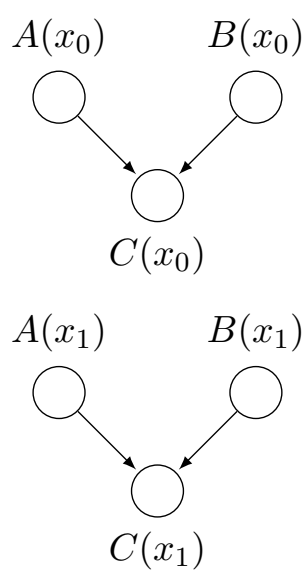

Figura 41: Representação da conjunção $C \equiv A \sqcap B$.

\section{A.4 Restrições universais}

Suponha que $|\mathcal{D}(y)|=n$. A restrição universal $\forall r(x, y) . C(y)$ é escrita como:

$$
\bigcap_{i=1}^{|\mathcal{D}(y)|}\left(r_{i} \rightarrow C_{i}\right)=\left(r\left(x, y_{1}\right) \rightarrow C\left(y_{1}\right)\right) \sqcap \ldots \sqcap\left(r\left(x, y_{n}\right) \rightarrow C\left(y_{n}\right)\right)
$$


Representação em RBN

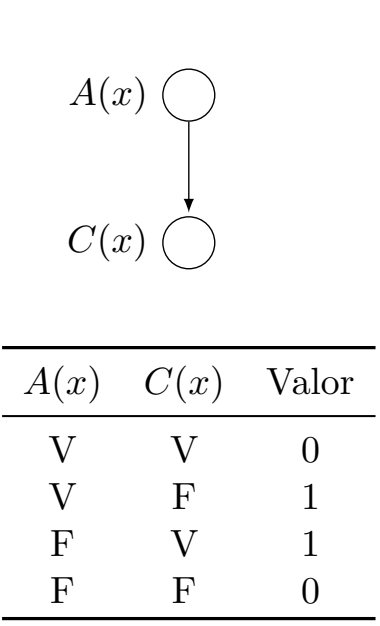

Rede proposicionalizada
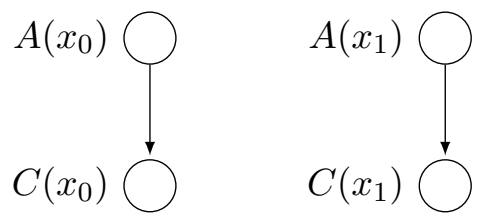

Figura 42: Representação da negação $C \equiv \neg A$.

A expressão $r_{i} \rightarrow C_{i}$ é logicamente equivalente a $\neg r_{i} \sqcup C_{i}$; esta relação é representada no nó auxiliar imply $(x, y)$. A Figura 43 ilustra a representação desta expressão.

\section{A.5 Restrições existenciais}

Suponha que $|\mathcal{D}(y)|=n$. A restrição existencial $\exists r(x, y) . C(y)$ é escrita na forma:

$$
\bigcup_{i=1}^{|\mathcal{D}(y)|}\left(r_{i} \sqcap C_{i}\right)=\left(r\left(x, y_{1}\right) \sqcap C\left(y_{1}\right)\right) \sqcup \ldots \sqcup\left(r\left(x, y_{n}\right) \sqcap C\left(y_{n}\right)\right)
$$

A relação $r_{i} \sqcap C_{i}$ é representada por um nó auxiliar and $(x, y)$. A representação é análoga à restrição universal e é apresentada na Figura 44. 


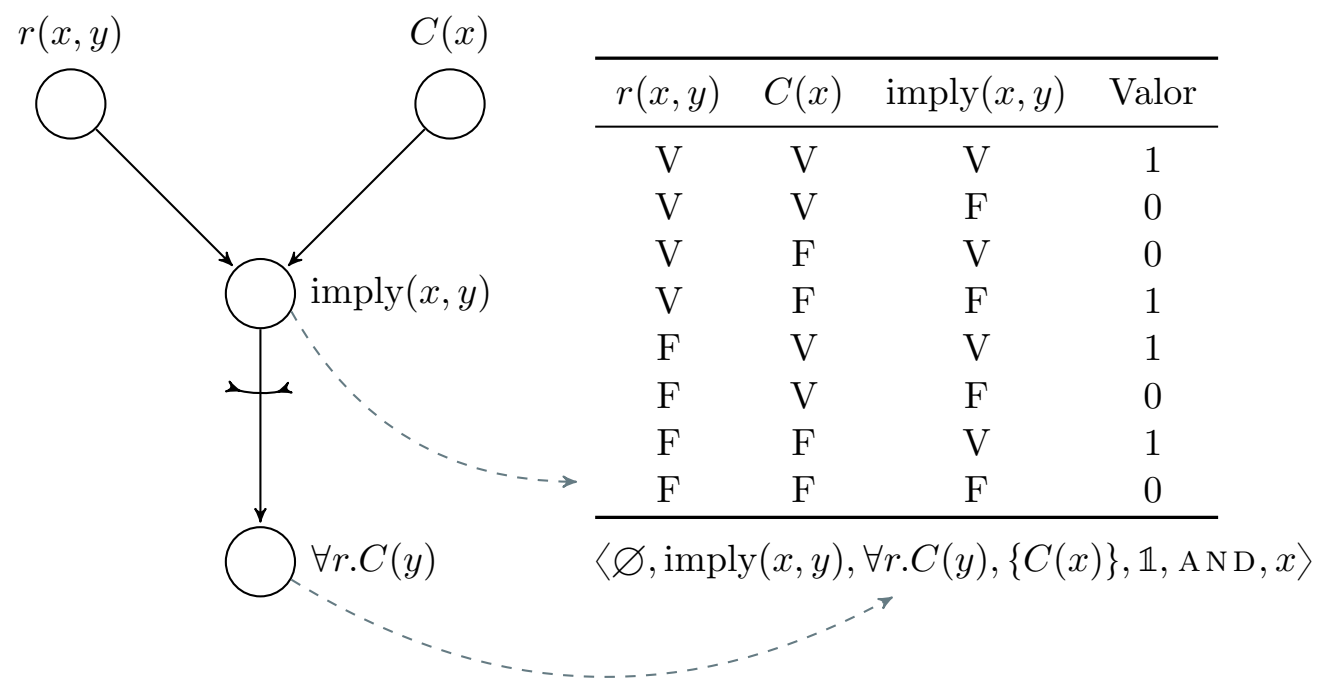

(a) Representação em RBN

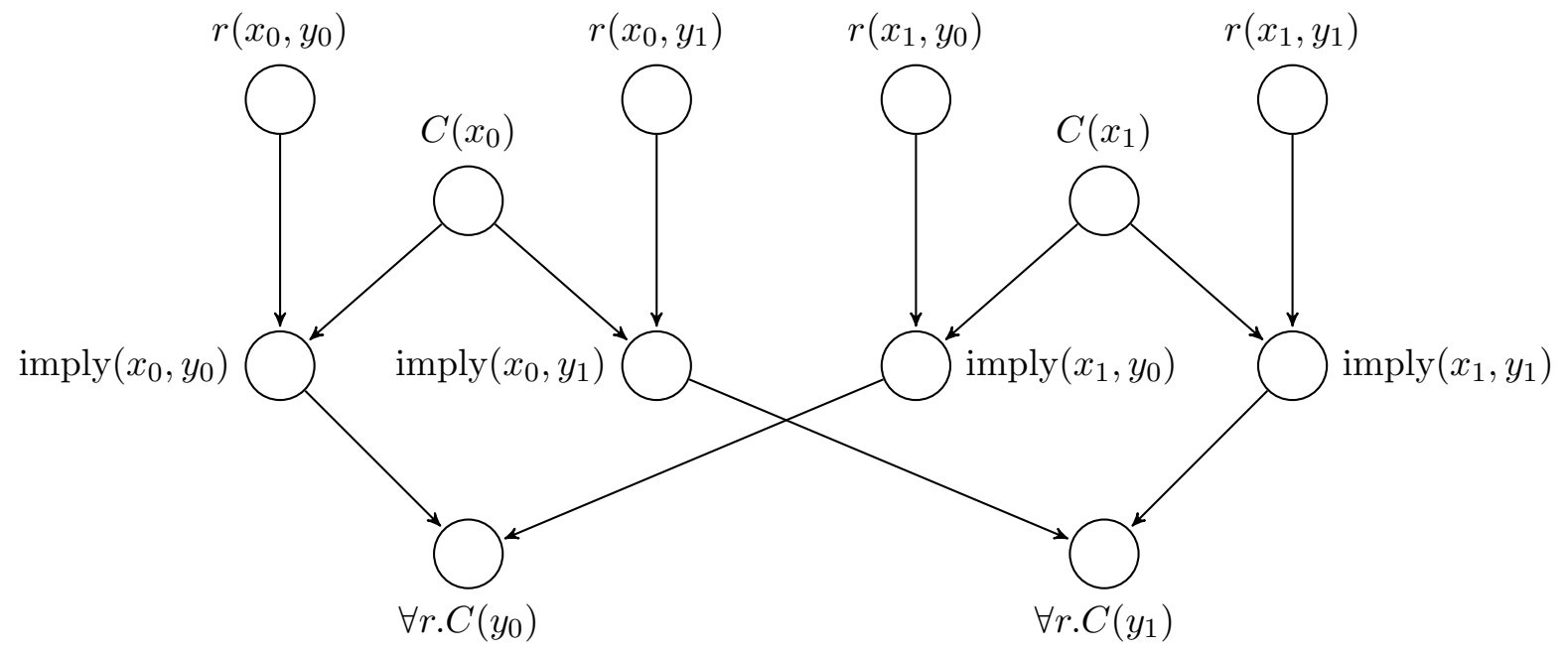

(b) Rede proposicionalizada

Figura 43: Representação da restrição universal $\forall r(x, y) . C(y)$. 


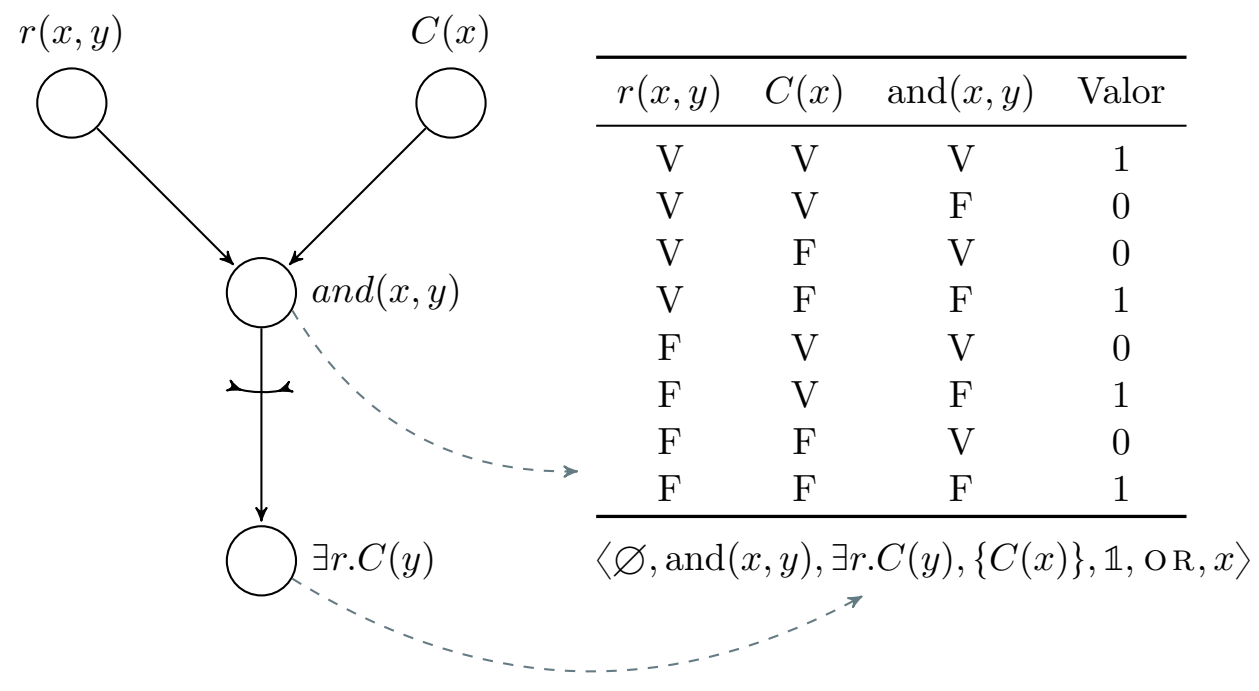

(a) Representação em RBN

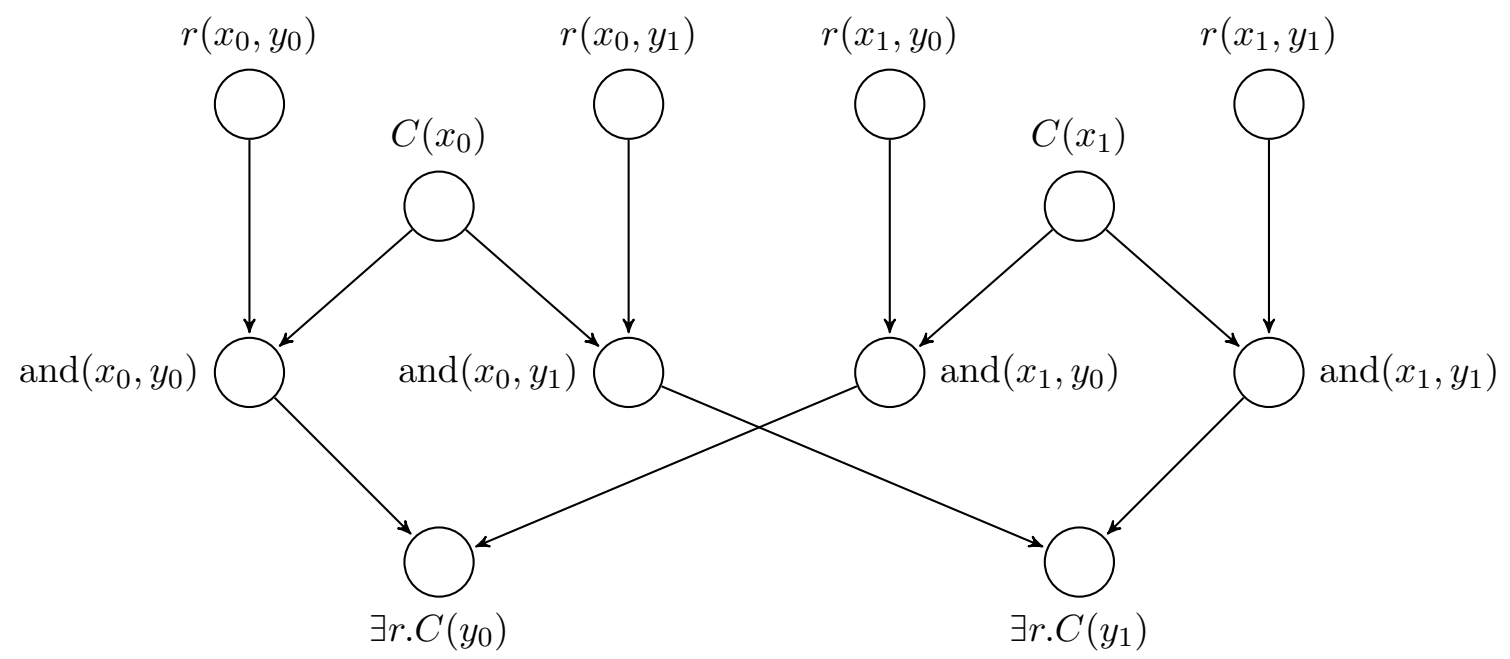

(b) Rede proposicionalizada

Figura 44: Representação da restrição existencial $\exists r(x, y) . C(y)$. 


\section{APÊNDICE B - Representação de fatores}

Nesta seção mostra-se como implementar um fator que seja espacialmente eficiente: para isso, sacrifica-se a expressividade em prol do tamanho da representação. As considerações realizadas a seguir foram propostas por Kisynski (2010).

Seja $\mathcal{D}(X)$ o domínio de uma variável aleatória $X$. Um fator $\mathcal{F}$ nas variáveis aleatórias $X_{1}, \ldots, X_{n}$ é uma função $\mathcal{F}: \mathcal{D}\left(X_{1}\right) \times \cdots \times \mathcal{D}\left(X_{n}\right) \rightarrow \mathbb{R}$. $\mathcal{F}$ representa uma distribuição de probabilidade conjunta nas variáveis $X_{1}, \ldots, X_{n}$.

Para representar de maneira eficiente um fator, as seguintes premissas devem ser verdadeiras:

1.Cada um dos conjuntos $\mathcal{D}\left(X_{i}\right)$ deve estar ordenado;

2.As variáveis aleatórias $X_{1}, \ldots, X_{n}$ devem estar ordenadas na definição do fator.

Desse modo, pode-se representar um fator como um vetor de valores. Seja $d_{m}$ o tamanho do domínio da variável $X_{m}$ e $x_{i_{m}}^{m}$ o elemento do domínio de $X_{m}$ que ocupa a posição $i_{m}$. Como pode ser observado na Figura 45 , o fator $\mathcal{F}$ possui $d_{1} \cdot d_{2} \cdots d_{n}$ tuplas cujos valores são $f_{0}, f_{1}, \ldots, f_{d_{1} d_{2} \ldots d_{n-1} d_{n}}$. Dada uma tupla $\left(x_{i_{1}}^{1}, x_{i_{2}}^{2}, \ldots, x_{i_{n}}^{n}\right)$, pode-se calcular o índice $k$ do valor associado a ela:

$$
k=\sum_{x=1}^{n}\left(\prod_{y=x+1}^{n} d_{y}\right) i_{x}
$$

Na Equação (B.0.1), quando $y=n+1$, considera-se que $\prod_{y=x+1}^{n} d_{y}=1$.

De maneira análoga, dado um valor $f_{k}$ do fator, pode-se recuperar o valor da tupla a partir do índice $k$ utilizando-se o Algoritmo 17. Note que uma tupla pode ser interpretada como um número numa "base variável", cujos algarismos são $x_{i_{1}}^{1}, x_{i_{2}}^{2}, \ldots, x_{i_{n}}^{n}$. Os procedimentos para se obter o índice a partir de uma tupla e uma tupla a partir de um índice 


\begin{tabular}{ccccc|l}
$X_{1}$ & $X_{2}$ & $\ldots$ & $X_{n-1}$ & $X_{n}$ & VALOR \\
\hline$x_{0}^{1}$ & $x_{0}^{2}$ & $\ldots$ & $x_{0}^{n-1}$ & $x_{0}^{n}$ & $f_{0}$ \\
$x_{1}^{1}$ & $x_{0}^{2}$ & $\cdots$ & $x_{0}^{n-1}$ & $x_{0}^{n}$ & $f_{1}$ \\
$\vdots$ & $\vdots$ & $\vdots$ & $\vdots$ & $\vdots$ & $\vdots$ \\
$x_{d_{1}-1}^{1}$ & $x_{0}^{2}$ & $\ldots$ & $x_{0}^{n-1}$ & $x_{0}^{n}$ & $f_{d_{1}-1}$ \\
$x_{0}^{1}$ & $x_{1}^{2}$ & $\cdots$ & $x_{0}^{n-1}$ & $x_{0}^{n}$ & $f_{d_{1}}$ \\
$x_{1}^{1}$ & $x_{1}^{2}$ & $\cdots$ & $x_{0}^{n-1}$ & $x_{0}^{n}$ & $f_{d_{1}+1}$ \\
$\vdots$ & $\vdots$ & $\vdots$ & $\vdots$ & $\vdots$ & $\vdots$ \\
$x_{d_{1}-1}^{1}$ & $x_{1}^{2}$ & $\ldots$ & $x_{0}^{n-1}$ & $x_{0}^{n}$ & $f_{2 d_{1}-1}$ \\
$\vdots$ & $\vdots$ & $\vdots$ & $\vdots$ & $\vdots$ & $\vdots$ \\
$x_{0}^{1}$ & $x_{d_{2}-1}^{2}$ & $\cdots$ & $x_{0}^{n-1}$ & $x_{0}^{n}$ & $f_{d_{1}\left(d_{2}-1\right)}$ \\
$x_{1}^{1}$ & $x_{d_{2}-1}^{2}$ & $\ldots$ & $x_{0}^{n-1}$ & $x_{0}^{n}$ & $f_{d_{1}\left(d_{2}-1\right)+1}$ \\
$\vdots$ & $\vdots$ & $\vdots$ & $\vdots$ & $\vdots$ & $\vdots$ \\
$x_{d_{1}-1}^{1}$ & $x_{d_{2}-1}^{2}$ & $\cdots$ & $x_{0}^{n-1}$ & $x_{0}^{n}$ & $f_{d_{1} d_{2}-1}$ \\
$\vdots$ & $\vdots$ & $\vdots$ & $\vdots$ & $\vdots$ & $\vdots$ \\
$x_{0}^{1}$ & $x_{d_{2}-1}^{2}$ & $\cdots$ & $x_{d_{n}-1-1}^{n-1}$ & $x_{d_{n}-1}^{n}$ & $f_{d_{1} d_{2} \ldots d_{n-1}\left(d_{n}-1\right)+d_{1} d_{2} \ldots\left(d_{n-1}-1\right)+\cdots+d_{1}\left(d_{2}-1\right)}$ \\
$x_{1}^{1}$ & $x_{d_{2}-1}^{2}$ & $\cdots$ & $x_{d_{n-1}-1}^{n-1}$ & $x_{d_{n}-1}^{n}$ & $f_{d_{1} d_{2} \ldots d_{n-1}\left(d_{n}-1\right)+d_{1} d_{2} \ldots\left(d_{n-1}-1\right)+\cdots+d_{1}\left(d_{2}-1\right)+1}$ \\
$\vdots$ & $\vdots$ & $\vdots$ & $\vdots$ & $\vdots$ & $\vdots$ \\
$x_{d_{1}-1}^{1}$ & $x_{d_{2}-1}^{2}$ & $\cdots$ & $x_{d_{n-1}-1}^{n-1}$ & $x_{d_{n}-1}^{n}$ & $f_{d_{1} d_{2} \ldots d_{n-1} d_{n}}$
\end{tabular}

Figura 45: Estrutura genérica de um fator

são análogos aos de uma conversão de base para números naturais. Ao utilizar este tipo de representação para fatores, não há necessidade de armazenar as tuplas em memória, somente seus valores.

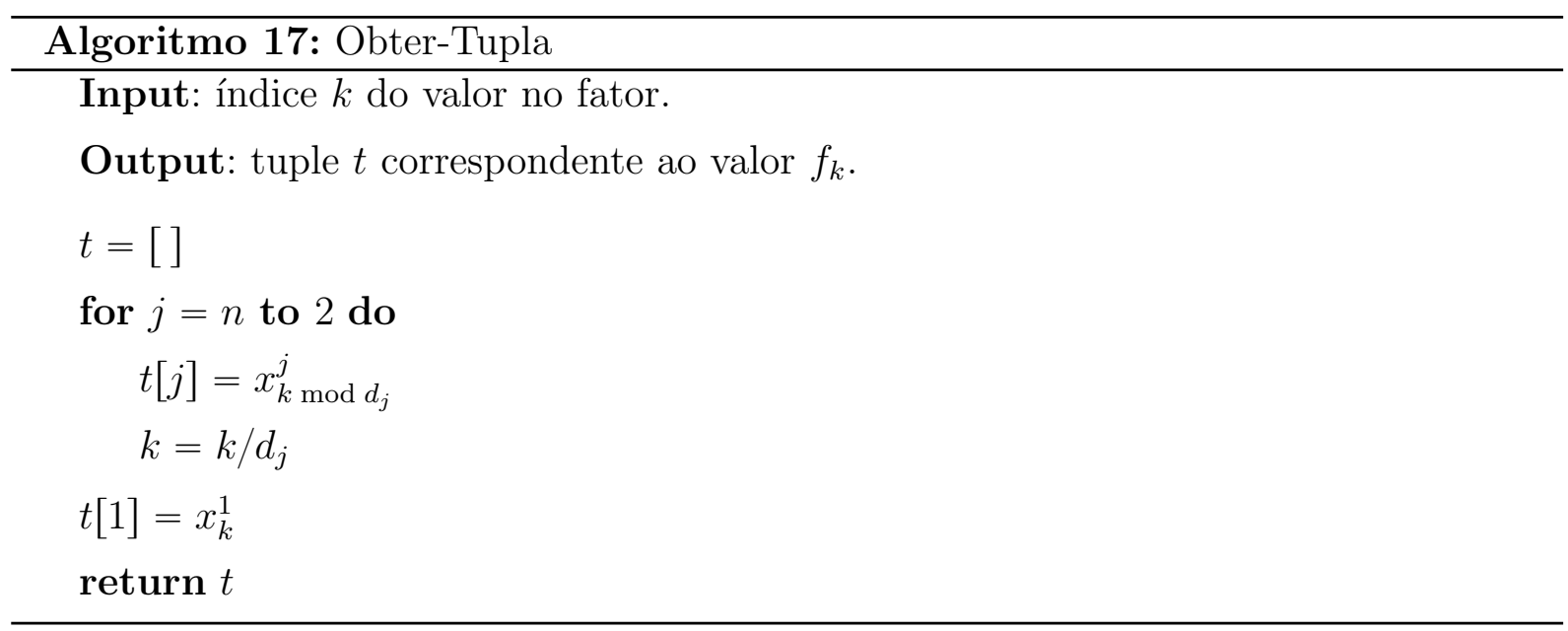

